NASA Technical Memorandum 4638

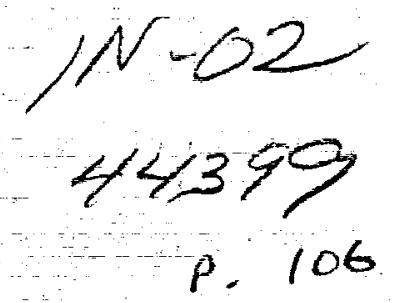

\title{
Experimental Results for a Hypersonic Nozzle/Afterbody Flow Field
}

Frank W. Spaid, Earl R. Keener, and Frank C.L. Hui

(NASA-TM-4638) EXPERIMENTAL

N95-23250

RESULTS FCR A HYPERSONIC

NOZZLE/AFTERBCOY FLOW FIELD (NASA.

Ames Research Center) $106 \mathrm{p}$

Unclas

$\mathrm{H} 1 / 02$

0044399

March 1995

NAXA

$y$

National Aeronautics and

Space Administration

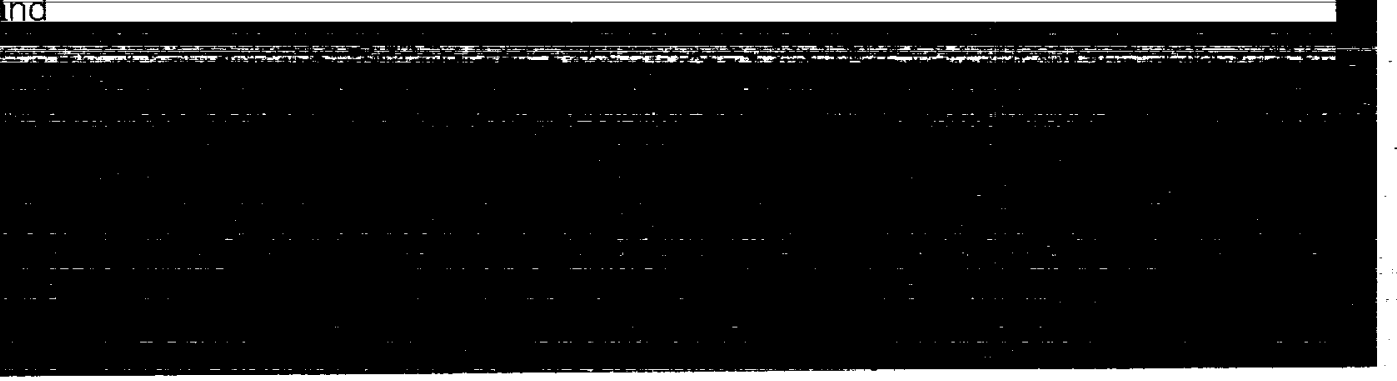




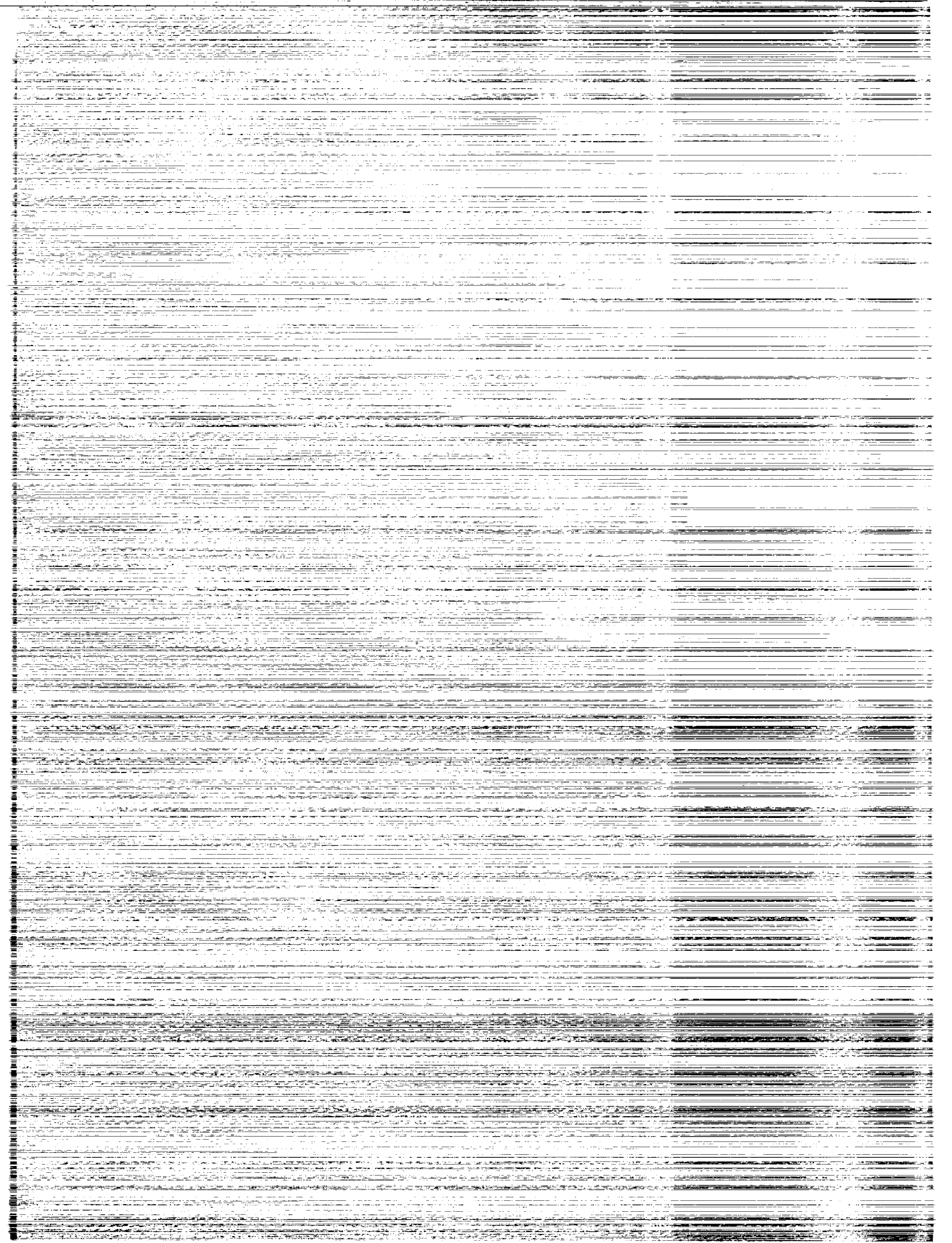


NASA Technical Memorandum 4638

\section{Experimental Results for a Hypersonic Nozzle/Afterbody Flow Field}

Frank W. Spaid, McDonnell Douglas Corporation, St. Louis, Missouri

Earl R. Keener, Eloret Institute, Palo Alto, California

Frank C. L. Hui, Ames Research Center, Moffett Field, California

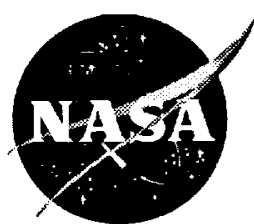

National Aeronautics and

Space Administration

Ames Research Center

Moffett Field, California 94035-1000 


\section{.}




\section{CONTENTS}

NOMENCLATURE

SUMMARY

I. INTRODUCTION

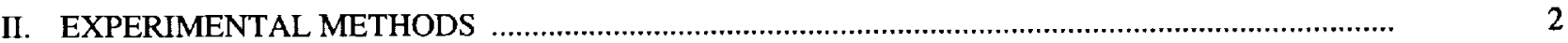

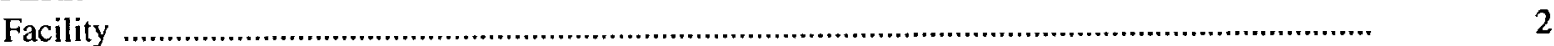

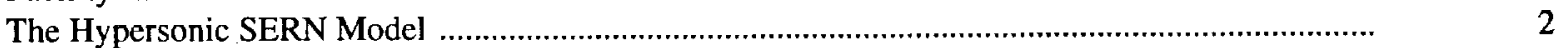

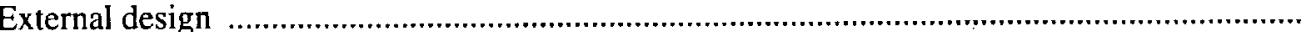

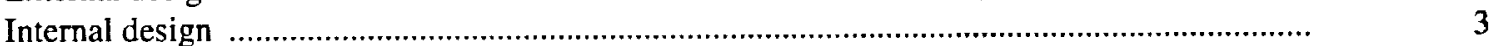

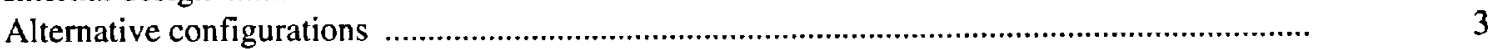

Instrumentation and Measurements

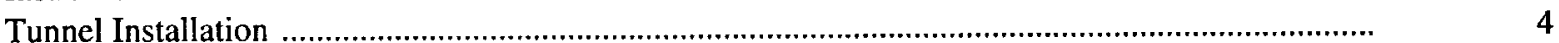

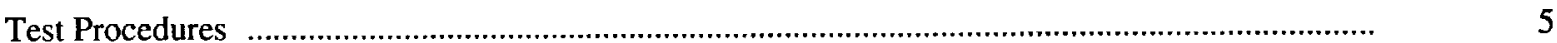

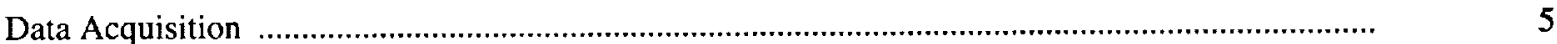

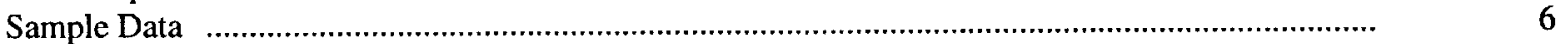

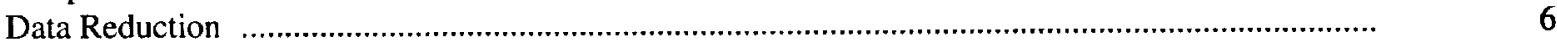

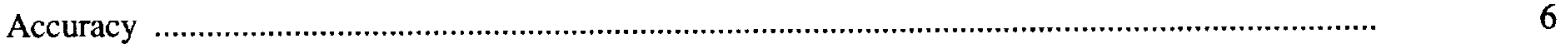

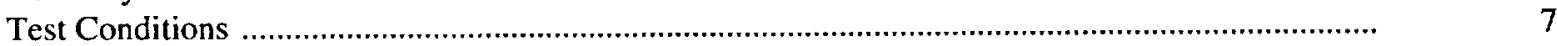

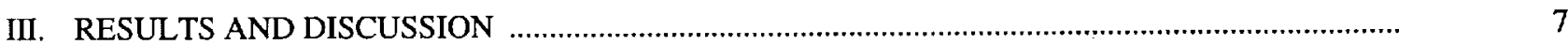

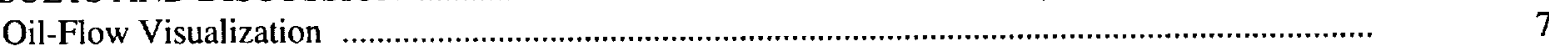

Baseline test condition

Effects of jet-pressure ratio

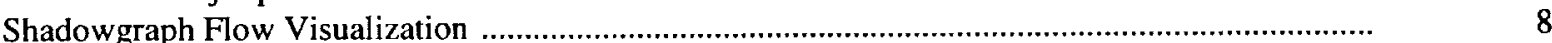

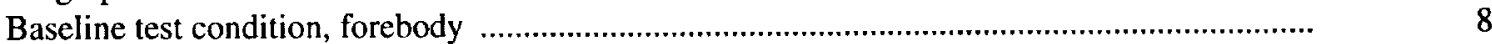

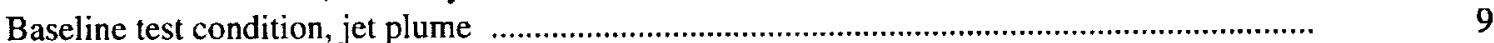

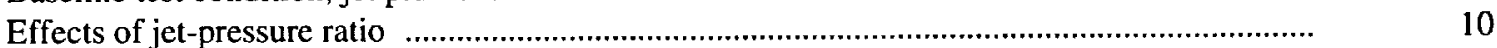

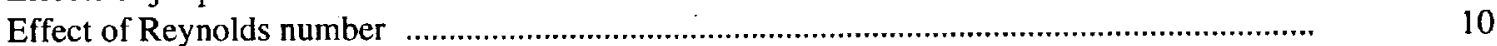

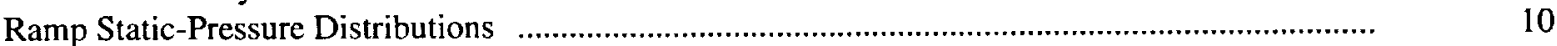

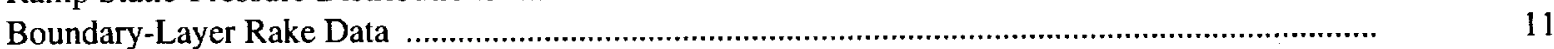

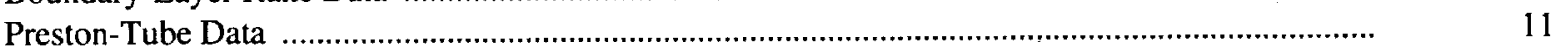

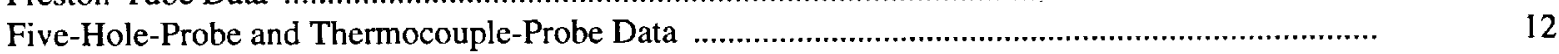

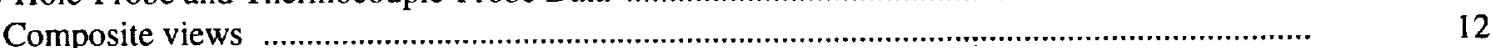

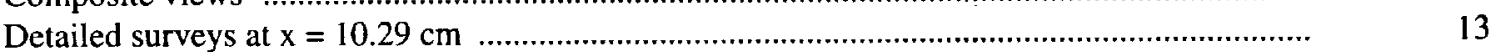

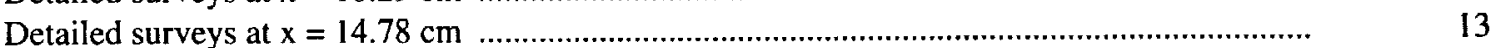

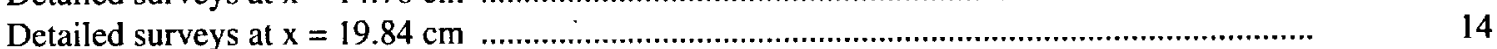

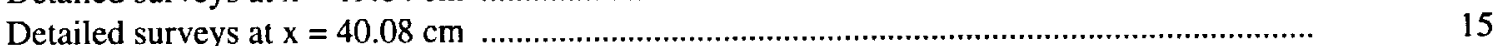

Probe and shadowgraph data comparisons

Sketches of the Jet/External-Flow Interaction

Shear-Layer Profiles ............................................................................................................

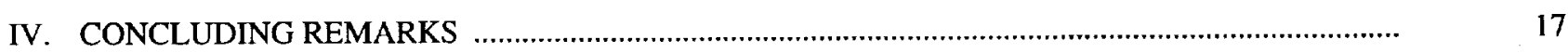

TABULATED DATA

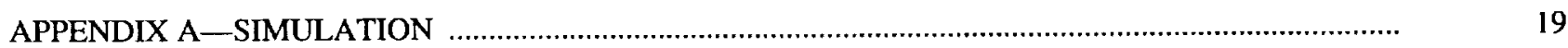

APPENDIX B_DESIGN OF INTERNAL NOZZLES .................................................................. 21

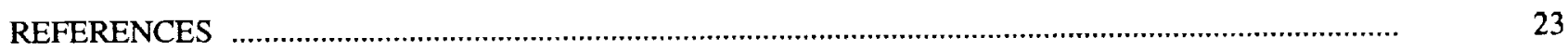

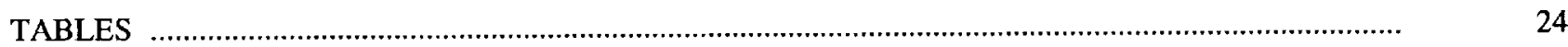

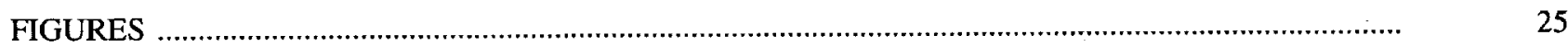

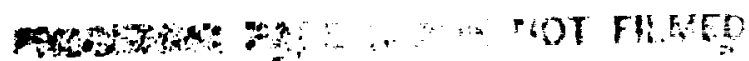

PAGE i INTENTIONÁLLY ELAAK 

NOMENCLATURE

$\mathrm{C}_{\mathrm{f}}$

d

$\mathrm{H}$

M

M

n

p

q

$\mathrm{Re}$

$\operatorname{Re}_{\theta}$

$\mathrm{T}$

u

$\mathrm{u}_{\tau}$

$\mathbf{x}$

$\mathrm{y}$

Z

$\alpha$

$\beta$ local skin-friction coefficient, $\tau / q_{e}$

Preston-tube diameter

boundary-layer shape factor, $\delta * / \theta$

Mach number

molecular weight

boundary-layer velocity profile

exponent

pressure

dynamic pressure, (1/2)pu ${ }^{2}$

unit Reynolds number

Reynolds number based on momentum thickness

temperature

velocity

shear velocity, $\left(\tau_{w} / \rho_{w}\right)^{1 / 2}$

streamwise distance from combustor exit station; positive downstream (fig. 3(c))

spanwise distance; positive right when facing in positive $x$ direction, origin at center of combustor exit station at inner surface of cowl (fig. 3(c))

vertical distance normal to $\mathrm{x}-\mathrm{y}$ plane; positive downward (fig. 3(c))

angle of attack

yaw-plane flow inclination angle; positive right when facing in positive $\mathrm{x}$ direction

$\tau$

0

2 ratio of specific heats

boundary-layer thickness

boundary-layer displacement thickness,

$$
\delta^{*}=\int_{0}^{\delta}\left[1-\left(\rho \mathrm{u} / \rho_{\mathrm{e}} \mathrm{u}_{\mathrm{e}}\right)\right] \mathrm{dz}
$$

$\theta$

boundary-layer momentum thickness,

$$
\theta=\int_{0}^{\delta}\left[\left(\rho \mathrm{u} / \rho_{\mathrm{e}} \mathrm{u}_{\mathrm{e}}\right)\left(1-\mathrm{u} / \mathrm{u}_{\mathrm{e}}\right)\right] \mathrm{dz}
$$

kinematic viscosity

density

local pitch-plane flow angle measured

in jet-plume $x-z$ coordinate system;

zero is parallel to the $\mathrm{x}$ axis;

positive is downward deflection

shear stress

\section{Subscripts}

refers to thermocouple probe corrected junction temperature

conditions downstream of a normal

shock wave

conditions at edge of boundary layer

jet flow conditions

nozzle coordinates (fig. 41)

nozzle profile coordinates (fig. 41)

refers to thermocouple probe support junction temperature

total conditions

conditions at the wall

free-stream conditions 



\title{
EXPERIMENTAL RESULTS FOR A HYPERSONIC NOZZLE/AFTERBODY FLOW FIELD
}

\author{
Frank W. Spaid, * Earl R. Keener, ${ }^{\dagger}$ and Frank C. L. Hui \\ Ames Research Center
}

\section{SUMMARY}

This study was conducted to experimentally characterize the flow field created by the interaction of a single-expansion-ramp-nozzle (SERN) flow with a hypersonic external stream. Data were obtained from a generic nozzle/afterbody model in the 3.5-Foot Hypersonic Wind Tunnel at the NASA Ames Research Center, in a cooperative experimental program involving Ames and the McDonnell Douglas Aerospace. The model design and test planning were performed in close cooperation with members of the Ames computational fluid dynamics (CFD) team for the National Aero-Space Plane (NASP) program. This paper presents experimental results consisting of oil-flow and shadowgraph flow-visualization photographs, afterbody surface-pressure distributions, rake boundary-layer measurements, Preston-tube skinfriction measurements, and flow field surveys with fivehole and thermocouple probes. The probe data consist of impact pressure, flow direction, and total temperature profiles in the interaction flow field.

\section{INTRODUCTION}

The National Aero-Space Plane (NASP) will rely on an airbreathing propulsion system during a major portion of its mission. This propulsion system will be based on scramjet engine technology. To contribute to the NASP research effort, the NASA Ames Research Center has undertaken a comprehensive experimental and computational investigation of selected generic components of the NASP configuration. An important aspect of the NASP technology development is the propulsion-system/ airframe integration. Accordingly, the Ames 3.5-Foot Hypersonic Wind Tunnel was used to conduct a series of tests on a generic nozzle/afterbody configuration.

Many difficulties associated with the development of a hypersonic vehicle arise from the fact that major portions of the flight environment cannot be simulated by existing ground-test facilities. Therefore, numerical

\footnotetext{
*McDonnell Douglas Corporation, St. Louis, Missouri.

†Eloret Institute, Palo Alto, California.
}

simulations of aerodynamic and propulsion flow fields obtained from computational fluid dynamics (CFD) codes will be used extensively to complement data obtained from experimental facilities. Confidence in predictions of the codes can be developed only by making detailed computational/experimental comparisons at conditions for which experimental data are available. The data sets used for these comparisons should represent the best that are available from existing experimental facilities with respect to accuracy, level of detail, and simulation of the flight environment. Predictions of the validated codes should then provide the most reliable estimates of the increments in performance or design parameters associated with the differences between the available test conditions and the flight environment. The purpose of this experiment is to experimentally characterize the flow field created by the interaction of the plume from a single-expansion-ramp nozzle (SERN) with a hypersonic external flow in sufficient detail to be adequate for CFD code validation.

A review of CFD validations experiments for hypersonic flows was presented by Marvin (ref. 1), in which both building-block and benchmark experiments are listed. The present experiment is listed as a recommended nozzle benchmark validation experiment in reference 1 . The test-section environment available in the 3.5-Foot Hypersonic Wind Tunnel is air with nearly perfect-gas properties. (Data including effects of gas chemistry will be obtained from experiments in impulse facilities, in which flight enthalpy levels can be approached or duplicated.) The Mach number range of 5 to 10 covers a large part of the hypersonic flight regime. The large test section allows testing of a relatively large model that can be adequately instrumented. The twominute (typical) run time and large model size allow detailed surveys of the jet/external flow interaction region. The combination of model scale and unit Reynolds number results in turbulent-boundary-layer flow on the model for a major portion of the operating envelope. A model was designed that would be acceptable for CFD code validation and hypersonic experimental research. All aspects of the test were planned with a view toward facilitating comparisons of the data with CFD predictions. The attempt to satisfy the requirements 
for a CFD validation experiment in the design of the model for this project has shown that the practical requirements of wind-tunnel model testing, such as finitethickness leading and trailing edges, can add complexity to the CFD effort by introducing extraneous effects into the experi mental flow field and by requiring modeling of regions of the flow field that are not related to the original objec tives. This added complexity may degrade the overall accuracy of certain computed solutions. Minimizing these undesirable features required close collaboration with those working on the CFD code development.

In the design of this experiment, significant departures from simulation of the flow field associated with a flight vehicle were made. First, the geometry was highly simplified, and second, the test gas was cold air. A more complete discussion of simulation requirements is given in appendix A. The simplified model geometry facilitated CFD computation of the model flow field and provided the added benefits of facilitating the flow-field surveys and reducing model cost. Because the jet gas was cold air, the jet temperature and velocity were low and the density was high relative to flight simulation requirements. The jet specific-heat ratio was 1.4 , which is higher than the range of values expected in flight. Important aspects of jet-plume/external-flow interactions were present in this experiment. Departures from simulation requirements for the flight environment were necessitated by the nature of the facility and by the available resources, and were justified because the primary test objective was to obtain data for CFD code validation.

This report presents experimental results consisting of oil-flow and shadowgraph flow-visualization photographs, afterbody surface-pressure distributions, rake boundary-layer measurements, Preston-tube skin-friction measurements, and flow-field surveys with five-hole and thermocouple probes. The probe data consist of impactpressure, flow-direction, and total-temperature profiles in the interaction flow field. Portions of these data and comparisons of experimental results with results of CFD computations have been previously reported (refs. 2-5).

This investigation is part of a cooperative program between McDonnell Douglas Corporation (MDC) and Ames Research Center, and was supported in part by the McDonnell Douglas Independent Research and Development Program.

The test was proposed by Gary Chapman, formerly at NASA Ames Research Center, now at the University of California, Berkeley. The work was supported in part by a grant from NASA to Eloret Institute. Our thanks also to Bill Lockman, Burt Uebelhoer, Mike Frediani, and Mike Reeves.

\section{EXPERIMENTAL METHODS}

\section{Facility}

The Ames 3.5-Foot Hypersonic Wind Tunnel is a closed-circuit, blow-down wind tunnel that has interchangeable, contoured, axisymmetric nozzles. Nozzles for test-section Mach numbers of 5.3, 7.3, and 10.3 are available. The test gas is air, which is heated by a storage heater containing aluminum oxide pebbles. Usable test time depends on test conditions, and varies from 0.5 to $4 \mathrm{~min}$. The test section consists of an open jet enclosed by a chamber $3.7 \mathrm{~m}$ in diameter by $12 \mathrm{~m}$ in length, arranged transversely to the flow direction, which contains the model support system and instrumentation. The available ranges of total pressure and total temperature are 690 to $12,400 \mathrm{kPa}$ and 667 to $1390 \mathrm{~K}$, respectively, although the usable ranges depend on the Mach number. To achieve high Reynolds numbers, the tunnel is operated at the minimum total temperature that will prevent liquefaction of the test-section flow.

\section{The Hypersonic SERN Model}

External design- The development of the concept for the generic SERN model is shown in figures 1 and 2. Figure 1 identifies the engine-airframe integration design criteria for hypersonic flight. Figure 2 is a sketch illustrating the nozzle model approach. The portion of the NASP vehicle modeled in this study is circled (the nozzle/afterbody region). The objective was to provide a wind tunnel model that would create a large-scale, nozzle-jet-plume flow over a representative afterbody.

The design procedure was an iterative process involving the overall model size, nozzle size, combustor station height and width (a representative internal nozzle station), nozzle throat height, cowl exit height and width, and afterbody ramp size. CFD computations played a major role in the model design process (see Ruffin et al., ref. 2).

The primary features of the model are shown in the schematic diagrams of figure 3 and the photographs of figure 4 . The model was designed to be the maximum size that can be accommodated by the facility at $M=10.3$. The large scale facilitates high-resolution experimental measurements, and promotes turbulentboundary-layer flow on the model. Except for the cowl, the side view of the model is a parallelogram. The forebody is a wedge whose upper surface is a flat plate with a nominally sharp leading edge $(0.12-\mathrm{mm}$ thickness). This relatively short flat-plate configuration was chosen with the intent of providing a nearly uniform external flow 
above the cowl, with a thin turbulent boundary layer at the cowl trailing edge. A thin boundary layer at the cowl trailing edge, relative to a characteristic vertical dimension such as the combustor exit height, is representative of a realistic configuration in which the cowl length is small with respect to the vehicle length. The 20-deg included angle of the leading edge was chosen as a compromise in order to minimize both tunnel-flow blockage and forebody length. A set of removable boundary-layer trips is provided for the forebody upper surface at a short distance downstream of the leading edge. The design and location of the trips are based on experimental data reported by Hopkins et al. (ref. 6) and Keener and Hopkins (ref. 7). The model leading edge is made of Invar to avoid warping caused by thermal stress. Most of the remaining model parts are made of 17-4PH stainless steel. The model is supported from below on a swept strut with a wedge-shaped leading edge.

Internal design- Air is supplied to a low-velocity plenum through a supply pipe in the model support strut. A perforated plate is located at the entrance of the supply pipe to the plenum (see figs. 3(a), 3(b), and 4(b)). The per forated plate lowers the pressure in the supply pipe by 76 percent through 128 square-edged orifices. Two screens located in the plenum were designed using wind tunnel flow-screen technology to smooth the flow from the perforated plate with negligible loss in total head. The internal surface of the cowl is flat, and interchangeable nozzle blocks can be mounted in the model between the plenum and the instrumented ramp (a single nozzle block was used in this experiment). The internal nozzle exit was intended to simulate a combustor exit station (see fig. 3(c)). The height of the combustor exit station, $2.03 \mathrm{~cm}$, was a compromise dictated in part by the construction tolerances of the throat height. The combustor-station height was chosen to be large enough so that reasonable resolution of the flow at this station could be achieved by probe surveys, and small enough to provide a moderately high aspect ratio at the combustor exit station.

The nozzles were designed by the method of characteristics, with a boundary layer correction, to provide uniform flow at the combustor exit station except for the wall boundary layers. For this test, the nozzle was designed for a combustor-exit Mach number of 1.75, which is intended to be representative of scramjet operation at the wind tunnel free-stream Mach number of 7.3. Pitot-pressure surveys were made at the combustor exit to assure that the flow was uniform at this station. Additional details concerning the internal nozzle design are presented in appendix $\mathbf{B}$.

The cowl and ramp are defined as starting where the combustor section ends. An arbitrary cowl length of $10.16 \mathrm{~cm}$ and a ramp angle of $20 \mathrm{deg}$ were chosen as a representative configuration (fig. 3(c)). A radius of $7.62 \mathrm{~cm}$ was chosen as a transition from the combustor exit station to the ramp to prevent boundary layer separation. A ramp length of $61.0 \mathrm{~cm}$ was chosen, using two-dimensional (2-D) CFD computations as a guide, which indicated that free-stream static pressure would be reached in the plume at this streamwise distance.

Alternative configurations- Various alternative configurations of the model were designed but were not constructed because of budgetary constraints (figs. 3(d) and 3(e)). These included side extensions, side fences to simulate 2D flow, two internal splitter plates to divide the nozzle into three "cngine-like" compartments, a heattransfer ramp, and hydrogen-gas gencrators to create a hot jet for heat-transfer measurements. A number of variations on the basic model configuration were considered because of the desire to obtain data corresponding to both 2-D and 3-D flows, and because the external flow along the sides and below the lower surface of the body alone would not be representative of the flow about a more realistic configuration, which would be considerably more slender. The configuration labeled "side extensions" in figure 3(d) was originally chosen as the baseline configuration, in an attempt to minimize the influence of the lower-surface forebody flow on the jet-plume/ external-flow interaction. In this configuration, the sides of the ramp were extended along the sides of the cowl and blended into the forebody upper surface through a 20-deg arc. A variation of this configuration, labeled "semispan model," was designed to allow a larger side extension, within the tunnel blockage restrictions. A CFD computation of the flow about the configuration with symmetrical side extensions by Ruffin et al. (ref. 3) showed that the side extensions led to large vortical structures above the ramp on either side of the jet plume. Observation of these vortical structures in the CFD simulation led to the selection of the "body alone" configuration as the baseline.

\section{Instrumentation and Measurements}

The jet total pressure and temperature were measured with two pitot tubes and a thermocouple in the plenum chamber downstream of the screens. The jet mass-flow rate was measured with an ASME orifice meter in the air supply pipe upstream of the model.

Two interchangeable ramp plates were tested downstream of the combustor exit station. A noninstrumented ramp plate was used for oil-flow visualization photographs. A second ramp plate was extensively instrumented with static-pressure orifices. Locations of the static-pressure orifices on the ramp and on the forebody of the model are shown in a plan view in 
figure 5. There are 120 static-pressure orifices on the ramp, forebody, and sides of the model. The pressures are measured by arrays of electronically scanned, solid-state transducers installed within the model (fig. 6). Shadowgraph photographs were obtained with both ramps.

During the initial phase of the test program, it was desired to make preliminary measurements of the boundary layers on the forebody and the ramp. Therefore, three small, fixed pitot rakes were located as shown in figure 5 - one on the forebody ahead of the plenum section and two on the ramp. A sketch of a typical rake is shown in figure 7 , and the probe locations are listed. These rakes are similar to those used successfully in flat plate boundary-layer studies to obtain velocity, displacement, and momentum thicknesses, to obtain the velocity power law, and to obtain skin friction from Clauser lawof-the-wall plots (ref. 8). Rakes 1 and 2 have probes made from $0.051-\mathrm{cm}$-diameter tubes soldered into $0.076-\mathrm{cm}$ diameter tubes, and have circular tips. The rakes are clusters of eight pitot probes distributed across the esti mated boundary-layer thickness (about $0.75 \mathrm{~cm}$ for rakes 1 and 2 and $1.2 \mathrm{~cm}$ for rake 3 ) and one probe at $2.5 \mathrm{~cm}$, well outside the boundary layer. The forward ramp rake is located about $10 \mathrm{~cm}$ behind the cowl, where a spanwise row of static-pressure orifices is also located. The rake is located outboard of a removable instrumented plug.

Skin friction was measured indirectly using Preston tubes, which are circular pitot tubes that are placed on the surface in a turbulent boundary layer. Preston-tube calibrations for supersonic and hypersonic flows are presented in references 9 and 10 . The calibration relates the pitot-tube pressure, diameter, and surface static pressure to surface shear stress through the law of the wall. The calibration correlates compressible measurements with the incompressible calibration through compressibility factors. The tube diameters selected were $0.157,0.239$, and $0.318 \mathrm{~cm}$, centered at $\mathrm{y}=2.38 \mathrm{~cm}$, and spaced $0.064 \mathrm{~cm}$ apart. The diameters were selected and the data reduced according to the recommendations of Hopkins and Keener (ref. 9). The reason for using three tubes is to ensure that the range of the calibration is covered. The calculated skin friction from all of the tubes should be the same, if none of the tubes is too small or too large. Skin friction was also measured directly on the ramp at two stations, with floating-element balances used in previous studies (refs. 6, 7, 9, and 10); however, it was found that the results were not usable because of uncorrectable effects of the strong pressure gradient.

Sketches and photographs of the five-hole and thermocouple probes used with the jet-plume traversing unit are shown in figure 8 . The labeling convention used for the five-hole-probe orifices is included in figure 8(a), and will be referred to in the section on data reduction. The designations $\mathrm{L}$ and $\mathrm{R}$ for the left and right tubes assume a view from above the probe, facing upstream. Two sizes of five-hole probes were built: $0.32 \mathrm{~cm}$ and $0.17 \mathrm{~cm}$ in diameter. The larger size was used, primarily because the computed response time of the larger probe and its connecting tubing was significantly shorter than that of the smaller probe. The larger size was considered to be acceptably small in comparison with the scale of the jet. The thermocouple probe is a type described by Kussoy et al. (ref. 11 ), and is sketched in figure 8(d). The Chromel-Alumel probe is a three-support unit with two junctions, as shown. The sensor junction is located at the center of the horizontal wire, and the other junction is located at the tip of one support. The probes are shown in figure 9 installed in a probe holder. During a survey, the probe tips were positioned at the same $\mathrm{x}$ and $\mathrm{z}$ locations, but the probe holder positioned the thermocouple probe $3.2 \mathrm{~cm}$ outboard of the five-hole probe.

The two-degree-of-freedom probe-traversing mechanism used for the probe surveys is shown in figure 10. The traversing unit is mounted above the model ramp. The probe holder is mounted on a horizontal circular tube which is attached to an air-cooled strut that has a wedge-shaped leading edge. The lower part of the strut that is immersed in the tunnel flow is swept. The upper part of the strut attaches to a positioning table that is remotely driven vertically by a motor-encoder assembly. The vertical positioning table is mounted, in turn, on a horizontal positioning table that is remotely driven in the cross-stream (y) direction. The mechanism is assembled within a rigid box structure; the horizontal positioning table is bolted to members that form the lower side edges of the box. Streamwise movement is accomplished by unbolting and reattaching the positioning-table assembly at the desired location between runs.

\section{Tunnel Installation}

Installation of the model and traversing unit in the tunnel test section is shown in figures 11-13. Figure 12 shows photographs of the model-alone installation, and figure 13 shows photographs of the model and traversing unit.

In figure 11, the two arms of the model-insertion mechanism are shown connected by a C-shaped structure (C-strut). The model support strut is attached to the lower arm of the insertion mechanism, and the traversing unit is attached to the C-strut near the upper arm. The entire apparatus can be pitched to change the angle of attack of the model. The degree to which this apparatus fills the 3.7-m-diameter test cabin is shown in the inset in figure 11. Before a run, the entire apparatus is retracted from the free-jet test section. After the flow is established, the model (in this case including the traversing unit) is 
inserted. The model is retracted before tunnel shut-down. Although the structure that contains the positioning tables and the motor-encoder assemblies is located above the test-section jet during a run, it is exposed to significant transient heating from the recirculating test-cabin flow. (Note that the bottom of the traversing-unit support structure is open to accommodate motion of the probe support strut.) Thermocouples were mounted at several locations on the traversing unit to monitor the status of this assembly during a run. Most of the structural temperature rise occurred after the run.

The air supply to the model nozzle is obtained from the Ames 3000-psi air supply system, through a regulator system that quickly stabilizes the jet flow at a preset total pressure. Figure 14(a) shows the first leg of the system from the connection to the 3000-psi air source (in the background), which is then connected to dome regulators. (Desired pressures are preset in the dome through a regulator-solenoid control system.) The next section is a 10.16-cm-diameter pipe (chosen to minimize pressure losses) containing a mass-flow, orifice-plate section (figs. 14(b) and 14(c)) with a differential-pressure transducer, a total-pressure transducer upstream, and a total-temperature transducer downstream of the orifice plate. The air supply pipe is then reduced to $5.08-\mathrm{cm}$ diameter pipe going up into the test cabin. Figure 14(d) shows the next section of the air supply, a high-pressure flexible hose to the model in the test cabin. A remote control panel was located in the tunnel control room, from which the jet total pressure could be preset before the jet air was turned on. The regulator was remotely activated either before or after the model was injected into the tunnel air stream.

Figure 15 is a shadowgraph of the jet flow with the cowl off. The shadowgraph shows the turbulent character of the jet. An oil-flow test on the ramp corresponding to this condition showed that the jet separates from the ramp shortly downstream of the nozzle exit. A system of shock waves is visible at the jet exit. Sound waves propagate from the jet through the ambient quiescent air. These waves can be seen most clearly in the upper right quadrant of the photograph. Sound propagation appears to be less intense in the direction opposite to the jet flow, as indicated by the much weaker wave pattern in the upper left quadrant of the photograph.

\section{Test Procedures}

For the main test program, the pebble bed in the wind tunnel heater was heated to obtain the desired tunnel total temperature. The air supply pressure to the model jet was preset to the desired pressure in the tunnel control room. The tunnel flow was started with the desired total pressure, which was remotely controlled to maintain a constant value. The model was inserted into the tunnel flow and the nozzle jet was started and remotely controlled by a regulator. For the ramp-pressure tests, several jet pressures could be set and recorded for each tunnel run (see the typical time history in fig. 16). For the jet-plume survey tests, the maximum run time of the tunnel was utilized to obtain one survey in either the vertical or the transverse direction. Surveys of the jet plume cross section were made at several streamwise stations from the cowl rearward. The surveys generally consisted of a lateral survey at constant height above the ramp or a vertical survey at the centerline of the ramp. Some vertical surveys were made off the centerline and also off the left side of the model. Some surveys extended through the model bow shock wave into the external tunnel flow field. The survey-point locations and spacing were selected to adapt to the flow field so more data were taken through the shock-wave and shear-layer regions. Repeat samples were taken at several data points during the return of the probe to the original position.

\section{Data Acquisition}

Test-section free-stream conditions, jet-stagnation conditions, jet mass-flow rate, rake-pressure data, Preston-tube data, and model static-pressure data were acquired by the NASA data acquisition computer. These data were transferred to both a NASA minicomputer and an MDC minicomputer for analysis. The traversing unit was controlled and the probe pressure and position data were acquired by the MDC minicomputer. A high-speed link between the two minicomputers allowed access to all of the test data through either machine. Data analysis codes and graphics software on both machines provided extensive quick-Iook data, and allowed data analysis to proceed in parallel with the data acquisition task.

Pressure- and temperature-probe data were acquired in two sets of files. The primary file contained values that were averages of samples obtained after the probe had been positioned at the desired location, and a predetermined time had elapsed to allow probe pressures and temperatures to stabilize. The auxiliary file contained time histories of pressure and temperature data at each location, beginning immediately after a desired probe position was reached. These auxiliary files were reviewed after each run to verify that the delay time used in data acquisition was adequate but not excessive. 


\section{Sample Data}

Figure 16 shows sample time histories of some of the data for a typical pressure run, including jet total pressure and selected static and rake pressures. The remotely controlled tunnel total pressure was nearly constant and the total temperature held to within about $30 \mathrm{~K}$. The jet control-system dynamics resulted in a slight overshoot at the beginning of each jet total-pressure setting (fig. 16(a)). Static pressure variations on the ramp correlated well with the changes in jet total pressure; static pressure lag was not apparent on this time scale. Several individual transducers sensed the instrumentcompartment pressure (fig. 16(g)). The pressures indicated by these transducers agree to within 0.006 psia ( 0.12 percent of full scale). The cause of the lowfrequency variations in some ramp boundary-layer rake pressures is not known. (The actual time history of these pressures is not accurately known because of the low data sampling rate.) Care was taken in reducing the ramp boundary-layer rake data to select an appropriate time interval for averaging these data. During another run, the jet total pressure was decreased continuously from 400 to $152 \mathrm{kPa}$, and it was found that there were no unusual pressure changes on the ramp.

\section{Data Reduction}

Compressible flow relations, including corrections for calorically imperfect gas effects (ref. 12), were used to reduce the data. These corrections were small at the baseline total temperature of $828 \mathrm{~K}$. Wind-tunnel free-stream conditions were calculated from the measured reservoir total pressure and temperature and from pressures measured by two pitot tubes mounted near the leading edge of the model. Since the free-stream static temperature and pressure were low, viscosity was calculated by Keyes' equation (ref. 13).

Five-hole-probe design, calibration, and data reduction followed the method outlined by Dudzinski and Krause (ref. 14). Calibrations were performed in the 3.5-Foot Hypersonic Wind Tunnel at the baseline test conditions, and in a free-jet calibration facility at MDC at Mach numbers of 2.5 and 3.5. Calibration data at the lower Mach numbers were obtained in a Reynolds number range representative of flow within the jet plume. The effect of Mach number on the calibration data was found to be small and was neglected. Data were obtained at a fixed set of flow conditions for pitch $(\sigma)$ and yaw $(\beta)$ angle ranges of $\pm 30 \mathrm{deg}$. The following parameters were computed from the probe pressure data (see fig. 8(a) for the probe-orifice nomenclature):

$$
\begin{aligned}
C_{a} & =\left(p_{d}-p_{u}\right) / d \\
C_{b} & =\left(p_{r}-p_{l}\right) / d \\
C_{t} & =\left(p_{t}-p_{c}\right) / d \\
d & =p_{c}-\frac{1}{4}\left(p_{l}+p_{r}+p_{u}+p_{d}\right)
\end{aligned}
$$

The pitch and yaw pressure-difference parameters, $C_{a}$ and $C_{b}$, were treated as independent variables; $\sigma, \beta$, and the total-pressure-correction parameter, $\mathrm{C}_{t}$, were treated as dependent variables. A surface-fitting routine was used to create interpolated and smoothed arrays based on the calibration data $\sigma\left(C_{a}, C_{b}\right), \beta\left(C_{a}, C_{b}\right)$, and $C_{t}\left(C_{a}, C_{b}\right)$. The effect of Mach number on the calibration data was found to be small, so the calibration data were assumed to be functions only of $C_{a}$ and $C_{b}$. Values of $C_{a}$ and $C_{b}$ were computed from the probe survey data, and an interpolation routine in the data reduction program was used to determine the corresponding values of $\sigma, \beta$, and $C_{t}$ from the calibration data arrays. The five-hole-probe data were reviewed to determine the applicability of the calibration data. Use of the calibration data implies that spatial variations in local flow-field properties are small relative to the probe height or width. This criterion is violated within the ramp boundary layer and in shock waves. In these instances, the impact pressure was assumed to be the maximum value measured by any of the probe ports, and the values of $\sigma$ and $\beta$ were discarded. A small number of total-pressure data points in any one survey include data reduced in this manner. The uncertainty of these data is estimated to be \pm 3 percent.

Conduction and radiation corrections were applied to the data obtained with the thermocouple probes, following the procedure described by Vas (ref. 15) using a datareduction program supplied by $C$. C. Horstman at NASA Ames. The conduction correction is a function of the Reynolds number based on the probe wire diameter and local flow conditions at the wire. The value of this Reynolds number remained above approximately 200 for the flow conditions of this experiment. In this Reynolds number range, the conduction correction function is not sensitive to variations in Reynolds number, so only a relatively crude estimate of the Reynolds number is needed.

\section{Accuracy}

The estimated probable uncertainties of pertinent recorded and calculated quantities are as follows: $\mathrm{M}_{\infty}, \mathrm{T}_{\mathrm{t}_{\infty}}, \mathrm{T}_{\mathrm{tj}}$, and $\mathrm{p}_{\mathrm{t}_{\infty}}, \pm 2$ percent; Re/m, \pm 8 percent; 
$\sigma, \pm 2$ deg; $\beta, \pm 3$ deg; probe $\mathrm{Pt}_{2}, \pm 1$ percent; model static pressures, $\pm 0.09 \mathrm{kPa}$; thermocouple probe temperature, \pm 2 percent at $T_{t_{\infty}} \pm 1$ percent at $T_{t j}$; probe location resolution, $\pm 0.15 \mathrm{~cm}$, position uncertainty, $\pm 0.25 \mathrm{~cm}$. These values were obtained from consultations with the staff of the 3.5-Foot Hypersonic Wind Tunnel, and from an error analysis of the data from this experiment.

\section{Test Conditions}

For the majority of runs, the model was mounted with its leading edge $3.8 \mathrm{~cm}$ downstream of the tunnel nozzle exit. A few runs were made, for the purpose of shadowgraph flow visualization of the leading-edge region, with the model installed with its leading edge $66 \mathrm{~cm}$ downstream of the tunnel nozzle exit. The forebody top surface was set at an angle of $-1.1 \mathrm{deg}$ (compression) with respect to the tunnel centerline. The model was set at this negative angle to increase the forebody top-surface pressure somewhat in order to counter the tendency of the top-surface flow on the wedge-shaped forebody to flow inward, which was reported in previous flat-plate studies with wedge-shaped leading edges (ref. 7). The oil-flow streaks on the forebody were streamwise so it was not necessary to run at a more negative angle of attack ( $-3 \mathrm{deg}$ was used in ref. 7 ).

The baseline test conditions established for this experiment are summarized in table 1 , which includes nominal values, variations among runs, and variations within runs. The axial Mach-number gradient was obtained from tunnel calibration data, and verified by data obtained from the model pitot tubes. The combustor exit Mach number and pressure ratio are representative of scramjet operation at the indicated value of free-stream Mach number. The jet was underexpanded at the cowl exit at the baseline jet pressure ratio of 310. Ramp pressure data were obtained at other pressure ratios and also at one-half the baseline free-stream total pressure.

Local Mach number on the forebody top surface was calculated from the free-stream Mach number, the tunnel total pressure, and the average of the forebody static pressures. The resulting value was 7.11 , which is in good agreement with the computed value corresponding to a 1.1-deg compression at the free-stream Mach number. A summary of forebody static pressure data including runto-run variations is presented in figure 17 . The computed value is included for comparison. Note that the run-to-run variations are on the order of \pm 0.25 percent of full scale of the low-range pressure transducers.

\section{RESULTS AND DISCUSSION}

\section{Oil-Flow Visualization}

Baseline test condition- Figure 18(a) shows results of an oil-flow test on the model cowl and ramp at the baseline jet-pressure ratio, $\mathrm{p}_{\mathrm{t} j} / \mathrm{p}_{\infty}$, of 310 at $\mathrm{p}_{\mathrm{t}_{\infty}}=6895 \mathrm{kPa}$ and $\mathrm{p}_{\mathrm{tj}}=374 \mathrm{kPa}$.

The oil-flow streaks that can be seen ahead of the cowl show that the flow into the jet is streamwise, as desired, at the model test angle of $-1.1 \mathrm{deg}$ (nose down). A transverse separation line is present on the upper surface of the cowl ahead of the trailing edge. The region of flow separation is indicated by the region of accumulated oil ahead of the trailing edge. This feature is consistent with the evidence of separation on the cowl that is seen in the shadowgraphs. The flow separation was expected because of the combination of curvature in the cowl exterior and the compression corner produced by the underexpanded jet.

The oil-flow pattern inside the cowl (not shown) was two-dimensional with no separation at the corners. The oil-flow pattern on the ramp is symmetrical with the centerline flow straight down the ramp. The surface flow direction turns outboard at the cowl trailing edge on each side. This outboard turning of the flow is a result of the lateral jet-flow expansion from the vertical cowl side trailing edge. The onset of outboard turning approaches the centerline as the distance down the ramp increases. (The white spots on the ramp appear to be small lumps of titanium-dioxide pigment.)

A symmetric pair of separation lines first occurs on each side of the ramp slightly outboard of the cowl exit and curves inboard, almost intersecting at the center of the ramp trailing edge. These separation lines are believed to be associated with the interaction of a shock wave, called the jet-plume internal shock wave (see the discussion of shadowgraph flow visualization), with the ramp boundary layer. The trajectory of this shock wave is undoubtedly influenced by the proximity of the model side edge. Outboard of these separation lines, the oil streaks flow inboard, indicating a vortical flow that probably fills in the flow around the ramp side edge. The side-edge effect is related to this particular model geometry. It appears that the aspect ratio selected for the nozzle was about minimum for the length of ramp chosen, since the separation lines for the jet extend the full length of the ramp before nearly meeting at the trailing edge.

Figure 18(b) shows an enlargement of the oil-flow photograph of the ramp in figure 18(a), with contour lines of constant flow angle superimposed. The outwardturning angles increase to more than $20 \mathrm{deg}$. The line for zero angle starts from the cowl trailing edge and sweeps 
inboard to the ramp centerline. This swept zero-angle line is related to the first-wave line of expansion of the lateral flow from the vertical trailing edge at the side of the cowl. Over the rearward two-thirds of the ramp, the zero-angle line is the ramp centerline. The flow angle increases with increasing spanwise and chordwise distance.

Figures 18(c) and 18(d) show the oil-flow pattern on the side of the model for full-length and closeup views, respectively, and figure 18(e) is a view of the oil-flow pattern from below. The oil streaks show that the flow around the lower model corner, from the high-pressure region on the lower surface to the lower-pressure regions on the side, does not result in significant upwash flow, but is nearly streamwise on the top half of the model. This is in contrast to subsonic flow, in which large upwash would occur. Disturbances on the lower half of the model are associated with the strut and do not appear to influence the ramp and jet flow field. A feature that appears to be a separation line originates at the jet exit and reaches the lower corner of the model upstream of the ramp trailing edge. This line is believed to be associated with the external jet-plume shock wave (seen in the shadowgraphs) that wraps around the side of the model.

Effects of jet-pressure ratio- Figures 19-21 show the results of oil-flow tests at off-design jet-pressure ratios $\left(\mathrm{p}_{\mathrm{tj}} / \mathrm{p}_{\infty}\right)$ of 154,234 , and 620 .

Figures 19(a)-19(e) show the results of an oil-flow test at a jet-pressure ratio of 154 (about one-half of the baseline value) at $\mathrm{p}_{\mathrm{t}_{\infty}}=6895 \mathrm{kPa}$ and $\mathrm{p}_{\mathrm{tj}}=186 \mathrm{kPa}$. The oil-flow pattern shows that the jet is separating in the cowl/ramp side comers. These flow disturbances are asymmetric and probably unsteady. The separated flow attaches on the ramp behind the heavy oil line and flows nearly straight to the ramp trailing edge. The extent of separation near the trailing edge of the cowl is less because of the reduced jet total pressure (fig. 19(d)). The jet-plume shock intersects the model side close to the ramp side edge (fig. 19(e)).

Figures 20(a)-20(d) show the results of an oil-flow test at a jet-pressure ratio of 234 (about $3 / 4$ of the baseline value) at $\mathrm{pt}_{\infty}=6895 \mathrm{kPa}$ and $\mathrm{ptj}_{\mathrm{tj}}=283 \mathrm{kPa}$. The cowl and side oil flows were obtained by applying two lateral and several vertical bands of oil. In figure 20 (a) the oil flow over the rear half of the ramp is not of good quality. However, near the cowl the oil-flow quality is sufficient to determine that the flow is attached, as can be seen in the closeup photograph, fig. 20(b). (The smeared oil in the left corner of the cowl is not part of the oil-flow pattern.) The right corner shows smooth, attached flow. The cowl closeup in figure 20(c) shows that the separated region ahead of the trailing edge is approximately the same length as for the baseline pressure ratio of 310 . The side view (fig. 20(d)) shows that the separation line for the jet-plume shock-wave/boundary-layer interaction is closer to the ramp edge than in the baseline case, as expected for the reduced blowing rate.

Figures 21(a)-21(c) show the results of an oil-flow test at an increase in jet-pressure ratio to 620 at $\mathrm{p}_{\mathrm{t}_{\infty}}=3447 \mathrm{kPa}$ and $\mathrm{p}_{\mathrm{tj}}=374 \mathrm{kPa}$. This test was made to determine whether the large increase in jet-pressure ratio would significantly affect the jet separation pattern on the ramp, perhaps moving the separation lines closer to the edges of the model. The photograph shows that the increase in jet-pressure ratio moved the separation lines out to the model edges on the forward part of the ramp, changing to about halfway to the edges on the rearward part. The streamwise separation distance near the trailing edge of the cowl increased because of the increased outward-flow-deflection angle at the higher pressure ratio (fig. 21(b)).

Figure 21(c) shows a side view of the model. The figure clearly shows the separation line, originating at the jet exit and reaching the lower corner of the model upstream of the ramp trailing edge, that is associated with the interaction of the jet-plume shock wave with the side of the model. This shock is farther forward of the similar line observed at the baseline jet-pressure ratio of 310 , as expected.

\section{Shadowgraph Flow Visualization}

The forebody and jet-plume flow field is clearly illuminated in the side-view shadowgraph photographs presented in figures 22-24 for both baseline and offdesign test conditions. Figure 22 presents shadowgraphs of the forebody at two Reynolds numbers. Figure 23 presents two shadowgraph photographs obtained at the baseline test conditions, one with the oil-flow (noninstrumented) ramp (fig. 23(a)), and one taken with the instrumented ramp (fig. 23(b)). Figure 24 presents several shadowgraphs taken at off-design test conditions, $79 \leq \mathrm{p}_{\mathrm{t} j} / \mathrm{p}_{\infty} \leq 620$.

Baseline test condition, forebody- Figure 22 shows shadowgraphs of the flow field over the forward part of the model for the unit Reynolds numbers of $14 \times 10^{6} / \mathrm{m}$ and $7.0 \times 10^{6} / \mathrm{m}$. The higher value corresponds to the baseline test conditions. There was some variation in Reynolds number between runs caused by variations in $T_{t_{\infty}}$; however, $p_{t_{\infty}}$ was closely controlled.

Features of the forebody flow field above the model and ahead of the jet plume include weak shock waves off the leading edge and boundary layer trips, transition in the forebody boundary layer, and boundary layer separation ahead of the jet plume. These forebody features will now be described; a description of the jet-plume flow field follows, in the next section.

Figure 22 shows that the forebody shock wave is weak above the top surface because the leading edge is 
relatively sharp and the angle of attack of the top surface is small. Weak shock waves occur off the boundary layer trips located $10.2 \mathrm{~cm}$ behind the leading edge. Results of a previous investigation (ref. 7) showed that identical trips did not adversely affect the downstream boundary layer characteristics at the same local Reynolds number, but caused earlier transition, as desired. The boundary layer trips were required because even the slight bluntness of the present model causes a profound increase in the length of laminar flow (refs. 6 and 7). It is important to know whether or not the forebody boundary layer is turbulent before it encounters the jet plume. The boundary layer on the top of the forebody is prominent in the shadowgraphs at the Reynolds numbers used in this test. The edge from the trips rearward is clearly highlighted by refracted light. The boundary layer and its edge look smooth directly behind the trips, but between the trips and the boundary layer rake it becomes ragged and looks turbulent. Actually, the complete transition process at Mach 7.3 takes place over an appreciable length that is almost equal to the length at the onset. Without further tests, it is not known what stage of the transition process corresponds to the onset of visible turbulence in the photograph. The appearance of transition onset for the baseline condition at $\operatorname{Re}_{\infty}=14 \times 10^{6} / \mathrm{m}$ in figure $22(\mathrm{a})$ is measured to be at $21 \mathrm{~cm}$ downstream of the model leading edge. This location is well forward of the cowl trailing edge, which was the objective of using the trips. The boundary layer thickness, as it appears in the shadowgraphs of figure 23 , grows to over $1.25 \mathrm{~cm}$ at the beginning of the cowl curvature.

Figure 23 includes two shadowgraphs of the aft part of the model which show the remainder of the flow field ahead of the jet plume. Figure 23(a) was obtained with the oil-flow ramp and figure 23(b) was obtained with the instrumented ramp. The shock wave that exists at the edge of the tunnel free-jet flow is seen near the top of the shadowgraphs. The tunnel jet flow expands slightly at Mach 7.3, and this shock wave exists where the outer part of the jet flow turns back slightly. A CFD study of this tunnel-jet expansion, resulting in a small axial-Machnumber gradient, concluded that its effect on the jet plume is negligible (ref. 3 ).

The forebody boundary layer thickens on the curved portion of the cowl and separates ahead of the cow] trailing edge, as expected, because of the compression corner caused by the plume shock wave and accentuated by the necessary external curvature of the cowl. The boundary layer passes through the jet-plume shock wave into the jet-plume shear layer.

Streamwise locations of the four probe survey stations are indicated by the vertical lines in figure 23(a); z-locations of the spanwise traverses are also shown.
Baseline test condition, jet plume- The shadowgraphs in figure 23 also show the jet plume and the interaction of the underexpanded jet with the external flow. Shadowgraph photographs were obtained with both the instrumented and the noninstrumented ramps. Features associated with the jet-plume flow field include expansion of the jet flow, the shear layer between the jet and the external flow, and the shock wave within the plume. These features are now described.

The jet-plume external shock wave is caused by the outward turning of the highly underexpanded jet flow as it leaves the cowl. The shape is dictated by the turning angle of the jet flow, which depends on the jet-to-ambient pressure ratio.

Underneath the jet-plume shock wave is the jetplume shear layer, which is formed by the difference in velocity between the jet and the external flow. The shear layer is prominent and highlighted above and below by refracted light, in a similar fashion to the edge of the boundary layer on the forebody. The shear layer can be seen to emanate from the cowl trailing edge as a thin, sharply defined layer that rapidly increases in thickness, then becomes diffused behind the ramp midpoint, and appears to be highly turbulent. The forebody boundary layer appears to merge with the shear layer above the cowl trailing edge and the separated-flow region. The forebody boundary layer probably has a significant effect on the subsequent development of the shear layer, and should be considered in attempts to compute this flow field.

An internal shock wave in the jet originates near the cowl trailing edge. It appears to be required in order to turn the flow parallel to the shear layer. This shock wave is believed to be analogous to the so-called "barrel shock" observed in underexpanded jets exhausting into a quiescent medium, and is also observed in jet- and rocket-exhaust flows.

As expected, there is a large expansion and turning of the flow as it leaves the cowl. The dark area near the cowl lip is possibly a region of high pressure and density gradients caused by the expansion. There is also a possibility that the dark area is created by light refracted off the cowl trailing edge, which may not be perfectly aligned with the shadowgraph light beam. There is also a small $(0.15 \mathrm{~cm})$ wake off the finite-thickness cowl trailing edge. The image lightens as the gradients decrease downstream. The expansion region from the cowl lip is bounded by the dark line of the internal shock and a white line, to be described next.

Under the expansion region emanating from the cowl trailing edge, there is a white line that also emanates from the cowl trailing edge and extends almost parallel to the ramp back to the boundary layer rake (seen in silhouette on the ramp surface). Apparently this line is the boundary 
between the expansion region influenced by waves originating from the cowl trailing edge and the expansion region emanating from the 20-deg radius at the ramp leading edge inside the cowl.

Between the white line and the ramp lies the expansion field that emanates from the 20 -deg radius at the ramp leading edge. Details of the expansion flow field will be discussed in a later section ("Sketches of Jet/External-Flow Interaction"). In this region, there can also be seen several weak shock waves radiating from inside the cowl, from the ramp leading-edge region. Most of these weak shocks (Mach waves) emanate from weak disturbances at the ramp and nozzle-block junction and at screw heads connecting the two model parts (although filler was used to cover the screw heads). The weak shock waves conveniently mark the wave angles of the expansion waves as the expansion waves intersect the white boundary line.

Just downstream of the cowl, a turbulent structure can be seen (more clearly in some photographs than others) which starts at the ramp surface and increases in thickness with increasing downstream distance. This turbulent structure is probably a side-edge vortex that is revealed in the oil-flow photographs described previously. These vortices fill in the flow between the side edge and the separation line.

Effects of jet-pressure ratio- Figures 24(a)-24(f) show the effects of varying the jet-pressure ratio, $\mathrm{p}_{\mathrm{tj}} / \mathrm{p}_{\infty}$, from 79 to 620 on the jet-plume flow field. Figures 24(a)-24(c) were obtained with the instrumented ramp at $\mathrm{p}_{\mathrm{t}_{\infty}}=6895 \mathrm{kPa}$ and $\mathrm{Re}_{\infty}=14 \times 10^{6} / \mathrm{m}$. Figures 24(d) and 24(e) were also obtained with the instrumented ramp, but at $\mathrm{p}_{\mathrm{t}_{\infty}}=3449 \mathrm{kPa}$ and $\mathrm{Re}_{\infty}=7 \times 10^{6} / \mathrm{m}$. Figure $24(\mathrm{f})$ is a shadowgraph obtained with the oil-flow ramp at $\mathrm{p}_{\mathrm{t}_{\infty}}=3449 \mathrm{kPa}$ and $\operatorname{Re}_{\infty}=7 \times 10^{6} / \mathrm{m}$

The internal shock wave, shear layer, and external shock wave are closest to the ramp in the immediate vicinity of the cowl trailing edge at the intermediate jetpressure ratio of 158 (fig. 24(c)) and move away from the ramp as the pressure ratio increases or decreases above or below this value. The oil-flow photographs probably explain the movement of the shocks and the shear layer away from the ramp in the immediate vicinity of the cowl exit as the jet-pressure ratio decreases below 158. At the lower jet-pressure ratio of 79 (and also at ratios as high as 158, fig. 24(c)) the nozzle flow is not full and flow separation occurs on the ramp at the cowl. However, the shadowgraph at the jet-pressure ratio of 79 (fig. 24(a)) does not show evidence of anything unusual in the jet flow. At twice the baseline jet-pressure ratio (fig. $24(\mathrm{e}), \mathrm{p}_{\mathrm{tj}} / \mathrm{p}_{\infty}=620$ ), the shocks and shear layer are forced outward.
The boundary-layer flow separation at the cowl trailing edge is greater at both low and high values of the jet-pressure ratio (figs. 24(a) and 24(f)) than at the baseline condition (fig. 23) because the initial jet-plume flow angles are larger.

Effect of Reynolds number-The principal effect of changing the free-stream Reynolds number at constant jet-pressure ratio (by changing the tunnel total pressure) is to change the location of boundary layer transition (fig. 22). Changing the Reynolds number does not noticeably affect the jet plume (constant $\mathrm{p}_{\mathrm{tj}} / \mathrm{p}_{\infty}$ ) nor the extent of forebody boundary-layer separation as long as the location of transition is far ahead of the cowl.

\section{Ramp Static-Pressure Distributions}

Ramp static-pressure distributions are presented in figure 25. Figure 25(a) shows the ramp surface-pressure distribution corresponding to the baseline test conditions. The distribution for the centerline, starting at the internal nozzle combustor station, is the most complete. The character of the centerline distribution is similar to that found in previous nozzle studies (refs. 16-18). The outboard pressure distributions show that the pressures decrease toward the model side edge as a result of the outward expanding flow. The pressures are equal on the two sides, indicating symmetrical flow.

A series of transverse ramp static-pressure distributions is presented in figure $25(\mathrm{~b})$. Data from two runs are superimposed, corresponding to $\mathrm{p}_{\mathrm{t} j} / \mathrm{p}_{\infty}=71.8$ and 603 , the limits of the range of pressure ratios explored in this study. The range represented by $p_{\infty}$ in . these coordinates is also shown. Note that the pressure distribution on most of the ramp is unaffected by variations in $\mathrm{p}_{\mathrm{tj}} / \mathrm{p}_{\infty}$. At the larger values of $\mathrm{x}$, an influence of pressure ratio appears on the sides, and apparently just reaches the centerline at $x=34.16 \mathrm{~cm}$. Only at the row of pressure orifices located farthest downstream, $\mathrm{x}=48.48 \mathrm{~cm}$, is the influence of variation in pressure present for all values of $y$.

The linear ordinates used in the preceding two plots de-emphasizes pressure variations at the downstream end of the ramp. Figure 25 (c) shows a comparison of data on the ramp centerline for the full range of pressure ratios using a logarithmic scale for the ordinate. Individual data points are omitted for clarity, and the range represented by $p_{\infty}$ is also shown. This comparison shows that the centerline pressure distribution is independent of pressure ratio for $\mathrm{x}<40 \mathrm{~cm}$. Farther downstream, the centerline pressure distribution is a function of pressure ratio, but the ramp pressure always expands below $\mathrm{p}_{\infty}$. 


\section{Boundary-Layer Rake Data}

A summary of boundary-layer rake data is given in table 2. Mach numbers were computed from rake pitot pressures and the local surface pressures by using the Rayleigh pitot formula with corrections for calorically imperfect gas effects (ref. 12). The forebody wall temperature was measured by a thermocouple installed at the forebody surface near the boundary layer rake. A value of $336 \mathrm{~K}$ was used for wall temperature in the forebody rake data reduction, which is the average of values obtained at the ends of several runs. The Crocco relation was used to compute the boundary layer totaltemperature distributions, as follows:

$$
\frac{\mathrm{u}}{\mathrm{u}_{\mathrm{e}}}=\frac{\mathrm{T}_{\mathrm{t}}-\mathrm{T}_{\mathrm{w}}}{\mathrm{T}_{\mathrm{te}}-\mathrm{T}_{\mathrm{w}}}
$$

The ramp boundary layers were assumed to be adiabatic with a recovery factor of 0.89 . This assumption was substantiated by measurements from thermocouples installed in the ramp surface.

The forward ramp rake is located $6.09 \mathrm{~cm}$ outboard of the ramp centerline (see fig. 5 and table 2). Streamwise rows of pressure taps are located near the rake spanwise station and on the opposite side at $y=6.88 \mathrm{~cm}$. The staticpressure data in figure 25 show that the rake is located in a significant cross-stream pressure gradient. The oil-flow photograph in figure 18(b) indicates that the surface flow direction at the rake location is greater than $20 \mathrm{deg}$ outboard. Data obtained with a five-hole probe at the boundary layer edge for the rake streamwise station but on the opposite side of the ramp indicated a yaw-plane flow angle of approximately $13 \mathrm{deg}$ outboard and a corresponding pitot-pressure error of 1 percent. Thus, a 1 percent correction (increase) was applied to pitot pressure data obtained from this forward ramp rake. The aft ramp rake is located only $2.86 \mathrm{~cm}$ outboard of the ramp centerline (see fig. 5 and table 2). The flow here is nearly streamwise (fig. 18(a)) and no correction was applied to the rake measurements.

Boundary-layer rake data (transformed by the Van Driest transformation (ref. 19)) are plotted in lawof-the-wall coordinates in figure 26. (See ref. 7 for a discussion of the method of presentation.) Values of skin friction used in computing the wall coordinates were obtained by the Clauser chart technique (ref. 8).

The range of $\operatorname{Re}_{\theta}$ for the forebody boundary layers is 3500 to 3700 . Values of the exponent, $n$, for the powerlaw velocity-profile representation

$$
\frac{\mathrm{u}}{\mathrm{u}_{\mathrm{e}}}=\left(\frac{\mathrm{z}}{\delta}\right)^{1 / \mathrm{n}}
$$

were found to be 12-14. A review of hypersonic boundary-layer data presented in reference 7 showed that in a plot of $n$ versus $\operatorname{Re}_{\theta}$ for $\operatorname{Re}_{\theta}<10^{4}$, the profile exponent shows considerable scatter and is considerably larger $(8<n<12)$ than the range of 6-7 which is typical of hypersonic turbulent boundary layers at higher Reynolds numbers. The present forebody boundary-layer data fall into this low-Reynolds-number category. Values of local skin-friction coefficient estimated from these data are $0.00127 \leq C_{f} \leq 0.00130$, which are somewhat greater than the value 0.0011 predicted by the Van Driest II theory (ref. 19). The momentum integral equation predicts that the boundary layer would grow by approximately a factor of two between the rake station and the beginning of the curved outer surface of the cowl, resulting in $\operatorname{Re}_{\theta} \approx 7400$ at this location. Since this value is still less than $10^{4}$, the entire turbulent portion of the forebody boundary layer is in the low-Reynoldsnumber range.

Boundary-layer momentum- and displacementthickness values obtained from the forward boundarylayer rake on the ramp from three runs at the baseline test conditions are compared with 2-D boundary-layer computations performed by the Cebeci-Smith method (ref. 20) in figure 27. The solid lines correspond to a computation in which transition was assumed to occur at the nozzle throat, and the dashed lines were obtained from a calculation in which transition was assumed to be at the combustor exit station, $x=0$. The data indicate that transition may have occurred in the vicinity of $x=0$. The significantly off-center location of the forward ramp rake and the 3-D nature of the flow near this rake make this conclusion somewhat tentative.

Velocity profile data from the aft ramp rake at two values of $\mathrm{p}_{\mathrm{t} j} / \mathrm{p}_{\infty}$ are presented in figure 28 . Comparisons among $p_{t j}$, the local surface pressure $p_{e}$, and the outerrake pitot pressure at this location are inconsistent with the assumption of isentropic flow from the jet plenum to the boundary layer edge at this location for all test conditions. Computation of $\mathrm{p}_{\mathrm{te}}$ from $\mathrm{p}_{\mathrm{e}}$ and the outer-rake pitot pressure (uncorrected for flow angle) results in $\mathrm{p}_{t e} \approx 0.42 \mathrm{p}_{\mathrm{tj}}$ at $\mathrm{p}_{\mathrm{tj}} / \mathrm{p}_{\infty}=312$. However, both the full profile and evidence from the oil-flow visualization photograph indicate that the rake was upstream of the jet plume internal shock wave at the baseline test condition. The distorted profile obtained at the lower pressure ratio is evidence that the rake pressures were influenced by the jet-plume internal shock wave at this test condition.

\section{Preston-Tube Data}

Table 3 presents skin-friction data obtained at the baseline test conditions from the three Preston tubes next 
to the forward ramp rake (fig. 5). The tube diameter selection and data reduction were made according to the recommendations of Hopkins and Keener (ref. 9). The objective of using the three tubes was to ensure that the range of the calibration was covered, thus providing a self-checking feature. The calculated skin frictions from the three tubes should be in agreement if the tubes are not too small or too large. For these data, the three tube diameters were about 31,42 , and 62 percent of the measured boundary-layer thickness. The smallest tube was the closest to the recommended size of $0.25 \delta$ (ref. 9). Since the Preston tubes were located closer to the model centerline than was the forward rake, the Preston-tube data were influenced less than the rake data by outboard flow. The values of $\mathrm{C}_{\mathrm{f}}$ in table 3 for each of the Preston tubes are in acceptable agreement, although the smaller tube gave slightly smaller values. Values of $C_{f}$ obtained from the Preston tubes differ from those obtained by the rake because the rake is located in a region of crossstream gradients and three-dimensional flow, and also because the rake is in a region of significantly lower static pressure. If rake pitot pressure data obtained from the tube near the wall are reduced using the Preston-tube data correlation, the results agree with values of $C_{f}$ derived by the Clauser method within 3 percent. Values of $C_{f}$ obtained from values of $\operatorname{Re}_{\theta}$ measured by the rakes and the Van Driest $\Pi$ theory are $0.00156 \leq \mathrm{C}_{\mathrm{f}} \leq 0.00193$, somewhat smaller than the values derived by the Clauser method (table 2).

\section{Five-Hole-Probe and Thermocouple-Probe Data}

Composite views- Figure 29 consists of two composite views of the jet-plume/external- flow interaction along the model centerline obtained from fivehole-probe and thermocouple-probe data. The data of figure 29(a) were obtained along the model centerline ( $x-z$ plane), and those of figure 29(b) were obtained from four spanwise traverses ( $x-y$ plane).

Features of the model geometry shown here include the cowl external contour, the jet flow passage beginning at the combustor exit station, and the ramp surface. Probe data are shown for the four axial stations surveyed in this experiment. The solid lines represent distributions of impact pressure, $\mathrm{p}_{\mathrm{t} 2}$, the local pitot pressure downstream of a normal shock corrected for the effect of misalignment between the probe axis and the local velocity vector. The thermocouple-probe data (dotted lines) are presented as the excess of the local total temperature above the value in the jet normalized by the overall temperature differ ence, $\left(T_{\mathfrak{t}}-T_{t j}\right) /\left(T_{1 \infty}-T_{t j}\right)$. Scales for these quantities are given in the inset at the top of the figure. Individual data points have been omitted from this figure for clarity. These data will be presented in greater detail in subsequent figures.

The $\mathrm{p}_{12}$ survey obtained at $\mathrm{x}=10.29 \mathrm{~cm}$ (fig. 29(a)) shows the outer portion of the ramp boundary layer, the variation within the jet resulting from the variation in local Mach number, the impact-pressure defect associated with the separated forebody boundary layer, and variations presumably associated with disturbances from the boundary layer trips and the leading-edge shock wave. No total-temperature data were obtained at this station.

Both impact-pressure and total-temperature distributions are presented at the three downstream stations, $x=14.78,19.84$, and $40.08 \mathrm{~cm}$. (The limited extent of the data corresponding to $x=14.78 \mathrm{~cm}$ is the result of a premature tunnel shut-down.) The value of obtaining both pressure- and temperature-probe data is illustrated by this figure, since it emphasizes the degree to which the two types of probes respond to different flowfield features. Discontinuities in $p_{12}$ caused by the shock wave within the plume and the external shock wave are evident in the profiles obtained at the three downstream stations (arrows). The locations and thicknesses of the shear layer between the jet and the external flow are clearly shown by the total-temperature distributions. A comparison of the impact-pressure and totaltemperature data shows that the aspects of the shear layer, originating with (a) the forebody boundary layer and its separation and (b) the interaction between the jet and the external flow, are independently identifiable, even at $x=40.08 \mathrm{~cm}$.

The character of the impact-pressure distribution is similar at the three downstream stations, showing a local minimum between the shocks in each case. These results suggest a degree of similarity of the impact-pressure distributions, if the separation between the shocks were used as a length scale and the local maximum impactpressure difference were used to scale the impact-pressure data. Note that there is no indication of shock waves in the total-temperature distributions, even though the local flow properties and the associated conduction corrections change significantly as the shocks are traversed.

At $x=14.78$ and $19.84 \mathrm{~cm}$, the minimum in the distribution of $\mathrm{p}_{\mathrm{t} 2}$ corresponds approximately with the center of the shear layer as indicated by the total temperature distribution. At $x=40.08 \mathrm{~cm}$, however, this feature of the impact-pressure distribution corresponds more nearly to the outer edge of the shear layer as indicated by the temperature profile. At this streamwise station, two sets of temperature-profile data are presented. These data are identical, except for an apparent z-position offset, and show two distinct layers with an intermediate region of approximately constant total temperature (fig. 29(a)). This type of temperature distribution is different from that obtained at the two upstream stations, 
and also different from all of the temperature distributions obtained from cross-stream ( $z=$ constant) surveys, to be presented later. The cause of this unusual temperature distribution in the $x-z$ plane at $x=40.08 \mathrm{~cm}$ is not known.

Figure 29(b) gives the corresponding plan view of the flow field. These traverses do not lie in the same plane, but were obtained at roughly comparable distances from the ramp surface; the data for $x=19.84$ and $40.08 \mathrm{~cm}$ correspond to the larger of the two $\mathrm{z}$ values for which data were obtained at these streamwise stations. The orientation of figure 29(b) is a mirror image of the actual experimental arrangement (positive $y$ is to the right, facing in the positive $x$ direction), and was chosen to facilitate comparison with the data of figure 29(a).

Many features of figure 29(a) are also present in figure 29(b): the separated boundary layer from the external surface of the cowl, the internal and external shock waves, and the proximity of the shear-layer location indicated by the total-temperature profile to the minima in the impact-pressure distributions. In the plan view, the jet impact-pressure profile is uniform at $x=10.29 \mathrm{~cm}$. Profiles of $p_{t 2}$ at the next two stations downstream clearly show the progress of the cowl trailing-edge expansion. Both the spanwise pressure profile and the temperature profile for $x=40.08 \mathrm{~cm}$ are significantly different from their counterparts in figure 29(a). The spanwise temperature profile does not show a two-layer structure. The internal shock wave is near the model centerline in the spanwise pressure profile, and the external shock wave does not appear as a sharp discontinuity.

Figures 30-33 show detailed distributions of impact pressure, pitch- and yaw-plane inclination angles, and total temperature. Both vertical $(y=$ constant $)$ and spanwise $(z=$ constant) surveys are included, and individual data points are shown. Complete temperature profiles are presented in physical coordinates.

Detailed surveys at $x=10.29 \mathbf{c m}$ - Profiles of $p_{t 2}$ and $\sigma$ obtained at $x=10.29 \mathrm{~cm}$ and $y=0$ are presented in figure 30(a). The impact-pressure data are also included in figure 29 , and the key features of this profile have already been discussed. Arrows indicate the ramp surface location in this and subsequent similar figures. The three data points nearest the surface in the $p_{t 2}$ profile are within the boundary layer. A dense spacing of data points was chosen near $z=0$ in an attempt to resolve the remnants of the cowl inner-surface boundary layer. Impact-pressure data for $-16<z<-7 \mathrm{~cm}$ lie between the separated forebody boundary layer and the shock from the leading edge. Variations in impact pressure in this region are approximately twice as large as variations measured at a single cross section in the tunnel calibration data, and are therefore believed to originate with the model. The outermost point in this profile (which consists of two data points) lies in the undisturbed test-section flow, above the leading-edge shock wave. These data and others like it were used in establishing the accuracy of the five-hole probe data.

Within the jet flow, the pitch-plane flow inclination profile varies in a piecewise linear fashion from near $20 \mathrm{deg}$ at the ramp boundary-layer edge to zero (one point shows a negative value) near $z=0$. The positive values of $\sigma$ in the viscous layer, $\mathrm{z}<0$, are consistent with the slope of the cowl outer surface. Above the viscous region, $\sigma \approx 0$, as expected.

Figure 30(b) presents $p_{t 2}, \sigma$, and $\beta$ distributions for $y=6.35 \mathrm{~cm}$. Results from two runs are presented-one that includes only data obtained within the jet. This run was terminated by a premature tunnel shut-down. There is a slight difference in $\mathrm{p}_{\mathrm{tj}}$ between the two runs, which is reflected in minor differences in the $\mathrm{P}_{\mathrm{t} 2}$ distribution, but repeatability of $\sigma$ and $\beta$ is good. Profiles of $p_{12}$ and $\sigma$ are similar to those obtained at $y=0$. The distribution of $\beta$ shows near-zero values throughout most of the profile, except near the ramp surface, where a slight inboard flow inclination is present.

Results of two spanwise five-hole-probe traverses at $\mathrm{x}=10.29 \mathrm{~cm}$ and $\mathrm{z}=2.54 \mathrm{~cm}$ are presented in figure 30(c). Flow properties are approximately constant within the jet $(\sigma \approx 13 \mathrm{deg}$ and $\beta \approx 0)$. The impact pressure distribution outboard of the cowl is similar to the profile obtained above the cowl (fig. 30(a)). The distributions of $p_{12}$ and $\beta$ show evidence of three weak shock waves in the external flow. The data points for $z>27.4 \mathrm{~cm}$ lie in the undisturbed test-section flow, outside of the model shock system. Values of $\sigma$ are small, except for a region of upward flow in the viscous region coming from the cowl outer surface. Differences in the $\sigma$ values between the two runs are most evident in this upflow region. However, the run corresponding to $\mathrm{p}_{\mathrm{tj}} / \mathrm{p}_{\infty}=315$ (triangular symbols) consists of a finely spaced set of data in which the probe moved in the positive y direction, followed by a sparse distribution of points as the probe returned to the model centerline at the end of the run. Data obtained in the upflow region just outboard of the cowl during the return path are in excellent agreement with data obtained at the same locations from the other run (circular symbols). A thin region of outboard turning is evident in the $\beta$ profile at the edge of the jet. This is qualitatively correct, since the flow is underexpanded, but may be partly a result of significant changes in flow properties near the cowl trailing edge at a distance on the order of the probe width. A region of inboard flow exists in the viscous region at the cowl outer surface, which is consistent with the cowl geometry.

Detailed surveys at $x=14.78 \mathrm{~cm}$ - Profiles of $\mathrm{P}_{\mathrm{t} 2}, \sigma$, and $\mathrm{T}_{\mathrm{t}}$ at $\mathrm{y}=0$ and $\mathrm{x}=14.78 \mathrm{~cm}$ are presented in 
figure 31(a). The impact-pressure and temperature data are also presented in the composite view in figure 29 . The impact-pressure data show a variation within the jet resulting from the Mach number variation, the discontinuities at the shock waves, the local minimum at the shear layer, and the gradient associated with the separated forcbody boundary layer. The lower portion of the profile will be reviewed later in connection with the discussion of the cowl exit expansion. The upper portion of the $\sigma$ profile shows evidence of the shock waves and variation within the shear layer. The total temperature distribution is constant within the jet, increases monotonically within the shear layer, and had just reached the test-section total temperature when the run was prematurely terminated.

Results of a spanwise survey at $x=14.78 \mathrm{~cm}$ and $\mathrm{z}=2.54 \mathrm{~cm}$ are presented in figure 31 (b). Profiles of $\mathrm{p}_{\mathrm{t} 2}$, $\sigma, \beta$, and $T_{t}$ are presented. These data were obtained at the same $z$ location as the data presented in figure 30(c), and the profiles show many similar features. The value of $\sigma$ is approximately constant within the jet flow, and $p_{t 2}$ and $\beta$ are approximately constant in the central region $(y<5.8 \mathrm{~cm})$ and then vary linearly until the internal jet shock is reached. The center is a region of 2-D expansion, influenced only by waves from the ramp and the inner surface of the cowl. The linear region is influenced by expansion waves from the cowl side edge. Evidence of the inner and outer shock waves and the shear layer is present in the distributions of $p_{12}$ and $\beta$, and indications of weak shock waves previously associated with the model flow field immediately downstream of the cowl exit are also present in the $p_{12}$ distribution. The wake of the cowl and upflow in the viscous region just outboard of the cowl can be seen in the $\sigma$ distribution. The total-temperature distribution was prematurely terminated by a broken wire before the outer edge of the shear layer was reached; data from the support temperature junction allows the position of the shear-layer outer edge to be estimated.

Detailed surveys at $x=19.84 \mathrm{~cm}$ - Distributions of $p_{t 2}, \sigma$, and $T_{t}$ at $x=19.84 \mathrm{~cm}$ and $y=0(y=3.2 \mathrm{~cm}$ for the $T_{t}$ distribution) are presented in figure 32(a). A single $p_{12}$ profile and a normalized version of the temperature data were presented and discussed in connection with figure 29 . The good repeatability of the $p_{t 2}$ distribution and the slightly poorer repeatability of the $\sigma$ distribution result from the fact that impact pressure involves a small correction to a primary measured quantity, but the flowdirection angle depends on the difference between two measurements of similar magnitude. These results show features that are similar to those observed in the profiles obtained at $x=14.78$. The two-slope nature of the $\sigma$ profile within the jet flow is still apparent, as is a local maximum in the profile that coincides with the local minimum in the impact pressure.
Figure 32(b) presents five-hole-probe data at the same streamwise station as the previous figure, but near the side of the model at $y=7.62 \mathrm{~cm}$. The qualitative features of the $p_{12}$ and $\sigma$ profiles are similar. The maximum value of the $p_{12}$ profile within the jet is smaller than the value measured at $y=0$ because of the expansion from the cowl side edge. This expansion is evident in the $\beta$ profile, which shows approximately $13 \mathrm{deg}$ outboard flow in the lower portion of the jet and minor variations in the shock/shear-layer region and becomes more streamwise with decreasing values of $\mathrm{z}$. No totaltemperature data were obtained at this survey station.

Figure 32(c) shows data from a five-hole probe survey at $x=19.84 \mathrm{~cm}$ and $y=11.43 \mathrm{~cm}$, which is $1.75 \mathrm{~cm}$ outboard of the model side. The lower portions of the profiles are characterized by low values of $\mathrm{p}_{\mathfrak{t} 2}$, slightly upward and inboard flow direction, and no discontinuities. The vicinities of the internal shock, the shear layer, and the external shock show small variations in $\mathrm{P} 2 \mathrm{2}$ and significant downward and outboard flow direction. At increasingly negative values of $z$, the impact pressure increases and the flow direction becomes slightly upward, with a near-zero value of $\beta$. The uppermost point in the profile shows a much larger value of impact pressure than the remainder of the profile--a value that indicates that the probe is within the shock system originating at the model forebody.

Figures 32(d) and 32(e) present data from two spanwise five-hole-probe surveys at $x=19.84 \mathrm{~cm}$ for two vertical locations, $z=1.77 \mathrm{~cm}$ and $5.58 \mathrm{~cm}$. Two runs with the five-hole probe and a total-temperature survey are included for the lower location (fig. 32(e)). The repeat runs at $\mathrm{z}=5.58 \mathrm{~cm}$ show the same general characteristics as the earlier repeat runs, in that impact-pressure data show less run-to-run variation than do flow-angle data. Comparison of these figures with the previous spanwise profiles shows the streamwise evolution of the jet-plume expansion, shock waves, and forebody flow field. The data in figure 31 (b) at $x=14.78 \mathrm{~cm}$ and similar data at $19.48 \mathrm{~cm}$ show a central region of the jet with constant properties and the influence of the cowl side-edge expansion, resulting in decreasing $p_{12}$ and increasing outboard flow direction with increasing $y$. The internal shock, the shear layer, and the external shock are most evident in the $\mathrm{p}_{\mathrm{t} 2}$ and $\sigma$ distributions. The pitch-plane flow inclination angle is small outboard of the external shock at both $\mathrm{z}$ locations and within the jet at $\mathrm{z}=1.77 \mathrm{~cm}$. However, the flow within the jet at $z=5.58 \mathrm{~cm}$ shows a significant downward velocity component resulting from increased proximity to the ramp. The total-temperature distribution at $\mathrm{z}=5.58 \mathrm{~cm}$ shows the usual two constant levels with a monotonic variation between. The shear layer indicated by the total-temperature distribution also 
coincides with the local minimum in the impact-pressure distribution, as observed previously.

Detailed surveys at $x=\mathbf{4 0 . 0 8} \mathbf{c m}-$ Data are presented in figure 33 (a) that correspond to $x=40.08 \mathrm{~cm}$ and $y=0$. The impact pressure and total-temperature data are included in figure 29; their primary features were discussed previously. Results from four five-hole probe surveys are included. The linear variation in $p_{t} 2$ and $\sigma$ within the jet is a prominent feature. Discontinuities in $p_{\mathrm{t} 2}, \sigma$, and $\beta$ at the shock waves, and variations associated with the shear layer are clearly evident. The significant outboard bias in the $\boldsymbol{\beta}$ profile is surprising, but is repeatable. The small values of $\beta$ observed at $y=0$ at the stations located farther upstream do not indicate a trend that would lead to the outboard flow shown in figure 33(a). The non-monotonic behavior and apparent $\mathrm{z}$-position shift in the total-temperature profiles shown in figure 33(a) were discussed in connection with figure 29. In addition, figure 33(a) shows a small difference in tunnel total temperature between the two runs presented.

Results of a five-hole-probe survey at the same streamwise station, but at $y=6.35 \mathrm{~cm}$, are presented in figure 33(b). The location of the total-temperature profile obtained during this run is $y=9.55 \mathrm{~cm}$. The impact pressure distribution shows lower values and less linear variation than the distribution obtained at $y=0$. For $z<0$, the differences between the $y=0$ profiles and these outboard profiles are substantial. The latter show no clear evidence of the internal shock in the $p_{t 2}, \sigma$, or $\beta$ results. The $p_{t 2}$ distribution in the shear layer is qualitatively different from that in the centerline profile, exhibiting a broad local minimum where the centerline data show a local maximum. There is also evidence of two external shocks in the $p_{12}$ distribution. The variations of $\sigma$ and $\beta$ at $y=6.35 \mathrm{~cm}$ are related to, but less abrupt than, variations in the corresponding profiles obtained at $y=0$.

Since the total-temperature probe is outboard of the five-hole probe, the total-temperature profile of figure 33(b) is only $0.13 \mathrm{~cm}$ inboard of the side of the model. This total-temperature survey shows that the jet flow does not cover the ramp at this streamwise station, but flow from the jet is present above the ramp. Two outer shear-layer edges are present in this profile, and the minimum temperature measured in this profile is only slightly greater than the jet total temperature, indicating that this profile is approximately tangent to the inner edge of the shear layer.

Figures 33(c) and 33(d) show results of spanwise five-hole- and thermocouple-probe surveys at $\mathrm{x}=40.08 \mathrm{~cm}$ for two $\mathrm{z}$ locations, $\mathrm{z}=6.85 \mathrm{~cm}$ and $11.67 \mathrm{~cm}$, respectively. Values of $\sigma$ are small at $z=6.85 \mathrm{~cm}$, but significant downward flow within the jet is evident nearer the ramp at $z=11.67 \mathrm{~cm}$.
Discontinuities in $\sigma$ and $\beta$ are more abrupt in the lower profiles $(z=11.67 \mathrm{~cm})$. The profiles of $p_{t 2}, \beta$, and $T_{t}$ at these two vertical locations show qualitative similarities, but corresponding features occur nearer the centerline in the lower profiles. The strength of the external shock, as indicated by the change in impact pressure, is approximately the same at the two vertical locations as it is in the vertical ( $y=$ constant) traverses. In both spanwise traverses, the center of the shear layer indicated by the total-temperature distribution is near the point at which the impact-pressure distribution begins to increase rapidly with increasing spanwise distance. Both of these spanwise total-temperature profiles show monotonic behavior, with no evidence of the additional structure observed in the vertical profiles at $y=3.2 \mathrm{~cm}$. The quantity plotted in figure 33(c) is actually the probe support temperature. The primary sensor wire had broken on a previous run, and an undamaged probe was not available for this run. Comparisons of the support temperature and the primary sensor junction temperature, to be presented later, show that the normalized support-temperature distribution is sufficiently close to the corrected center-junction temperature to be a less accurate, but useful, indication of the shear layer profile.

Probe and shadowgraph data comparisonsFigure 34 is a comparison of locations of the jet-plume internal and external shock waves determined by measurements from a shadowgraph photograph (fig. 23(b)) with those from five-hole-probe data. Vertical distances were measured from the forebody upper surface; horizontal distances were measured from the cowl exit station. Lengths measured from the photograph were converted to physical lengths by multiplying them by the ratio of the actual model height, $25.4 \mathrm{~cm}$, to the height of the model image. This procedure produced reasonable agreement between the image of the ramp surface and its known location, but significant differences are indicated between the shock locations observed in the shadowgraph and those from the probe data. Effects of errors related to the shadowgraph were explored, such as differences between shadowgraphs and errors in the apparent locations of model surfaces caused by imprecise shadowgraph alignment. The most likely error in shock position indicated by the probe data is believed to be probe support deflection caused by aerodynamic loading. The loading on the probe holder and support in the vicinity of the shocks would produce an upward deflection. Corrections for this type of probe support deflection would be negative increments in $\mathrm{z}$, which would increase the discrepancies between the two types of data. No satisfactory explanation for the observed discrepancies has been found. 


\section{Sketches of the Jet/External-Flow Interaction}

Figure 35 shows a vertical cross section of the model and jet plume at the plane of symmetry, including a sketch of the nozzle internal contour. Wave lines, representing expansion waves, emanate from the 20 -deg radius beginning at the end of the internal nozzle (the combustor exit station) as the jet expands onto the ramp. The first three wave lines in the sketch intersect the inside of the cowl, while downstream wave lines exit the cowl and interact with the expansion field beginning at the cowl trailing edge and the shear layer.

The white line that appears in shadowgraph photographs (fig. 23) beginning at the cowl trailing edge and extending almost parallel to the ramp is believed to be the first expansion wave from the cowl trailing edge. This conclusion is supported by the pitch-plane flowangle distributions obtained from the five-hole-probe surveys. For example, see figure 31(a). The initial change in slope of the pitch-plane angle distribution within the jet flow in figure 31 (a) occurs at $z=2.4 \mathrm{~cm}$, which is in good agreement with the location of the white line at this streamwise station from the shadowgraph photograph of figure 23 , and is also in good agreement with the location of the intersection of a Mach wave corresponding to Mach 2.2 extended from the cowl trailing edge.

In figure 35 , it can be seen that the interaction of the expansion waves from both the ramp leading-edge region and the cowl trailing edge causes them to bend away from the jet-flow primary direction. The interaction of the ramp leading-edge expansion waves with the shear layer causes the shear layer to bend toward the ramp. This effect demonstrates the importance of the relationship of the cowl trailing edge to the ramp leading edge and its effect on the shape of the jet plume where it leaves the cowl trailing edge.

The extent of the region where the flow over the ramp is independent of the details of the interaction with the external flow is determined by the distance required for expansion waves from the cowl trailing edge to reach the ramp. The white line conservatively represents the boundary of the region of isolation, although the isolated region also includes the region of expansion of the flow from the cowl trailing edge before the expansion waves reflect back to the ramp. Thus, much of the forward ramp surface flow is independent of the flow characteristics at the edge of the jet, as indicated by the static-pressure distributions presented in figure 25 .

The remainder of the sketch (fig. 35) depicts the shadowgraph results: inner and outer shock waves, jet shear layer, forebody boundary layer, and separation near the cowl trailing edge. Flow streamlines are also shown, which depict the inner flow turning down the ramp and the outer flow turning outward and then turning back slightly through the internal shock wave.

Figure 36 is a sketch of a horizontal cross-sectional view through the jet. The lateral flow to the side must be similar to the centerline vertical flow (fig. 35) except that there are no expansion waves from the ramp leading edge in the horizontal plane. The flow is streamwise from the cowl until it encounters the lateral expansion waves from the cowl vertical trailing edge and turns outward. The boundary for the outward expansion must be similar to the white boundary line in the vertical plane. This boundary must curve laterally within the jet plume and end at the ramp. At this boundary, the flow begins to turn outward from the center of the jet plume. In the oil-flowangle sketch in figure $18(\mathrm{~b})$, the contour where the flow begins to turn outward (the zero-angle line) must be this boundary, the outward flow being associated with the expansion region from the cowl trailing edge. Within this boundary is a conical-shaped region where the flow is expanding two-dimensionally as a result of the 20 -deg turning angle for the ramp.

The sketch in figure 37 shows a transverse cross section near the cowl where 2-D ramp flow exists over part of the ramp span, outboard of which the flow turns outward. A boundary area can be seen separating the two previously discussed regions of expanding flow: the inner region of 2-D expanding flow from the ramp leading edge and the outer region of 3-D expanding flow from the cowl trailing edge. Downstream of the conical line for zero flow angle (fig. 18(b)), the flow expands three-dimensionally outward from the center of the jet plume. The jet does not expand smoothly to the side edge of the ramp, but separates (S) because of the influences of the model side edge and the internal shock within the jet. The shear layer extends above and outboard of the cowl location, but it is likely that the shear layer merges with the corner flow. The inner shock wave lies inside the shear layer and probably induces the jet-flow separation on the ramp. According to the transverse components of the oil streaks outside of the separation line, vortices must exist on each side edge of the model, emanating from the separation lines $(\mathrm{S})$ on the model side and ramp surfaces (as depicted in fig. 36), to accommodate the corner flow. The outer shock wave surrounds the jet plume, which expands upward and outward from the cowl trailing edge, and ends at the model side where the oil-flow-separation line appears.

Shock-wave and shear-layer locations obtained from the probe data are shown in cross-section views in figure 38 for the three downstream survey locations. Few surveys were obtained at $x=14.78 \mathrm{~cm}$, and the data are insufficient to allow sketching the intermediate locations of features with reasonable confidence. More data were obtained at $x=19.84$ and $40.08 \mathrm{~cm}$, however, and 
shock-wave and shear-layer locations have been sketched to aid in interpretation of the data. Estimates of ramp boundary-layer thicknesses obtained from the rake data are also included at these downstream stations. The shear layer, as indicated by the temperature-probe data, nearly fills the region between the internal and external plume shocks at the two upstream stations, but these features become more widely separated at $x=40.08 \mathrm{~cm}$. The shape of the internal shock wave changes significantly between the two downstream stations, and the shear layer is clearly seen to approach the ramp inboard of the ramp side edge in figure 38(c).

\section{Shear-Layer Profiles}

Figure 39 includes plots of sensor and support temperature distributions through the shear layers, plotted as the normalized temperature difference,

$$
\frac{T-T_{t j}}{T_{t \infty o}-T_{t j}}
$$

These plots are scaled to show the shear-layer shapes more clearly than those presented previously, and they also show the degree to which the normalized support temperature distribution approximates the normalized sensor temperature distribution.

Shear-layer thickness data obtained from the plots of normalized temperature distribution are shown in figure 40. The shear-layer inner and outer edges are defined as follows:

$$
\frac{T_{t}-T_{t j}}{T_{t \infty}-T_{t j}}=0.05,0.95
$$

At the two upstream stations, the shear layers are seen to be significantly thicker at the sides than near the centerline, but this trend is reversed at the downstream station. The flow at $x=40.08 \mathrm{~cm}$ is highly $3-D$, and the shear-layer thickness results can perhaps best be interpreted by reference to the cross-section view, figure 38(c).

\section{CONCLUDING REMARKS}

Data were obtained from an experiment conducted with a generic nozzle/afterbody model in the Ames 3.5-Foot Hypersonic Wind Tunnel to experimentally characterize the flow field created by the interaction of a SERN flow with a hypersonic external stream. The model design and test planning were performed in close cooperation with members of the Ames NASP CFD team. This paper has presented experimental results consisting of oil-flow and shadowgraph flow-visualization photographs, afterbody surface-pressure distributions, boundary-layer rake measurements, and five-hole- and thermocouple-probe surveys.

The design, construction, and operation of the nozzle/afterbody model was successful. The internal nozzle gave the desired flow to the cowl and afterbody sections. Surface oil-flow patterns show where the jetplume flow is attached to the afterbody surface at jet pressure ratios (ratio of jet total pressure to free-stream static pressure) of 150 or more. The oil streaks show the region of large outward turning of the underexpanded jet just behind the cowl, giving a highly 3-D boundary layer. The oil flow also shows the pattern of lines where the jet flow separates from the ramp, apparently as a result of interaction of the jet-plume internal shock wave with the ramp boundary layer. The flow is entirely attached to the ramp at the cowl exit station, and the separation lines approach the model centerline near the afterbody trailing edge. Between the jet-separation lines and the afterbody side edge is a vortex flow field that accommodates the corner flow to the main flow field. The jet plume is clearly illuminated in shadowgraph photographs, which show the flow-field characteristics of the jet plume and the forebody. Features associated with the flow ahead of the jet plume are (1) shock waves that emanate from the model leading edge and from the boundary layer trips; (2) a turbulent boundary layer on the forebody, starting ahead of the forebody midsection; and (3) boundary layer separation slightly forward of the cowl trailing edge, caused by the effective compression corner produced by the curvature of the cowl and the large expansion angle of the jet. Features associated with the jet plume include (1) the shock wave in the external flow that results from the expansion of the jet flow; (2) the shear layer between the jet and the external flow; (3) the shock wave within the plume; and (4) the boundary within the plume between two expansion regions: (a) the two-dimensional, 20-deg expansion at the ramp leading edge and (b) the outward expansion of the flow from the cowl trailing edge. Boundary-layer rake data indicate that the turbulent portion of the forebody boundary layer is of the low Reynolds-number type. Ramp rake data indicate that boundary layer transition may have occurred near the combustor exit station at the baseline test conditions. This conclusion is tentative, however, and future experiments, such as boundary layer surveys at the cowl exit station, would aid in determining the location of transition for the internal nozzle and ramp flow field.

The static-pressure distribution normalized by the jet total pressure is independent of the overall pressure ratio 
for a major portion of the ramp surface. Five-hole- and thermocouple-probe surveys were conducted at four streamwise stations. The impact-pressure distributions are complex as a result of multiple shock waves and the large differences in both reservoir pressure and temperature between the jet and the external flow. A comparison of the impact-pressure and total-temperature data shows that aspects of the shear layer that originate with the fore body boundary layer and the interaction with the jet and the external flow are independently identifiable for a considerable distance downstream of the cowl exit Iocation. Cross-section views of shock-wave and shearlayer locations constructed from the probe data show that the flow is highly 3-D throughout the region of investigation. Shear-layer thicknesses obtained from thermocouple-probe surveys show significant differences between vertical and spanwise traverses.

\section{TABULATED DATA}

Tabulated data and detailed model geometry from this experiment can be obtained by contacting the Reacting Flow Environments Branch, NASA Ames Research Center. 


\section{APPENDIX A-SIMULATION}

The present experiment is part of a series of experiments that are intended to provide information on SERN flow fields for the NASP application. The testsection environment available in the 3.5-Foot Hypersonic Wind Tunnel limited the present experiment to the study of flows of perfect gases with nearly constant properties.

The requirements for dynamic and thermal similarity between a flight condition and a wind-tunnel test of a powered model are obtained by nondimensionalization of the Navier-Stokes equations and boundary conditions. If consideration is restricted to flows of perfect gases, and if molecular diffusion at the shear layers between the jet and the external flow is neglected, this procedure leads to the following simulation requirements:

(1) geometric similarity

(2) duplication of $M_{\infty}, \operatorname{Re}_{\infty}, \gamma_{\infty}, T_{w} / T_{\infty}(x / L, y / L, z / L)$

(3) duplication of $\gamma_{j}, \mathrm{p}_{\mathrm{j}} / \mathrm{p}_{\infty}$, and $\left(\mathrm{T}_{\mathrm{j}} M_{\infty}\right) /\left(\mathrm{T}_{\infty} M_{\mathrm{j}}\right)$

Values of the test-section Mach number and Reynolds number that can be achieved in the 3.5-foot wind tunnel are representative of points on a NASP-type trajectory. In addition, simulation of the Reynolds number and the wall temperature distribution is not critical for flows in which viscous-inviscid interaction, wall boundary-layer transition, and separation do not play an important role. It is difficult to satisfy the requirements pertaining to the jet flow in a hypersonic wind-tunnel test of a NASP-type vehicle, since the jet gas must have a high temperature (or a low molecular weight) and a specific-heat ratio of somewhat less than 1.4. For example, if testing is conducted at $\mathrm{M}_{\infty}=7.3$ and $\mathrm{T}_{\mathrm{t}_{\infty}}=830 \mathrm{~K}$, which is approximately the minimum value that will prevent condensation in the test section, then for $M_{\mathrm{j}} \approx 29, \mathrm{~T}_{\mathrm{tj}} \approx 1900 \mathrm{~K}$ is needed to satisfy the above requirements. Testing with jet gases in this temperature range would have greatly increased the cost and complexity of the test, and would have precluded obtaining data of the desired detail and accuracy. The parameter $\left(\mathrm{T}_{\mathrm{j}} M_{\infty}\right) /\left(\mathrm{T}_{\infty} M_{\mathrm{j}}\right)$ can be simulated at a low jet-stagnation temperature with gas mixtures such as helium-argon, but at the price of a large mismatch in $\gamma_{j}$.

Results of previous studies - for example, Cubbage et al. (ref. 16) - have indicated that important features of SERN flows can be duplicated without simulating the dynamics of the shear layer between the jet and the external flow. If the requirement for simulating the velocity and density ratios across the shear layer are omitted, then parameters related to jet flow simulation reduce to the jet specific-heat ratio and jet pressure ratio $\mathrm{p}_{\mathrm{j}} / \mathrm{p}_{\infty}$. Computations performed by Oman et al. (ref. 17) for a scramjet operating at $M_{\infty}=8$ indicate that the $\gamma$ of the combustion products would vary significantly during the expansion process, and that both the average value of $\gamma$ and its variation would have a large effect on a SERN pressure distribution. It was concluded that the use of a simulant gas mixture, such as argon and Freon, could closely approximate the variation of $\gamma$ in the jet plume (see Pittman, ref. 18). Moderate heating of the argon-Freon mixture used in the study of reference 18 was needed to avoid condensation in the plume. It may not be possible to use gaseous mixtures containing Freon compounds in future tests because of the apparent environmental hazard associated with release of these compounds into the atmosphere. The use of this technique with large wind tunnel models and at higher free-stream Mach numbers presents formidable practical problems, because of the need for safe gas mixtures and elevated temperatures.

The current test plan included significant departures from the previously discussed simulation requirements. First, the geometry is highly simplified, and second, the jet gas is cold air. As a result, the jet temperature and velocity are low, and the density high, relative to flight simulation requirements. The jet specific-heat ratio is 1.4 , higher than the value expected for a flight vehicle. These departures are justified by the argument that the primary test objective was to obtain data for CFD code validation. 



\section{APPENDIX B-DESIGN OF INTERNAL NOZZLES}

The initial design of the inviscid nozzle contours was performed by a method developed by Foelsch (ref. 21). The nozzle flow up to the inflection point on the nozzle wall is approximated as a source flow; downstream of the source-flow region, the flow is a simple-wave flow, and the calculation is exact. A cubic curve is used to connect the nozzle throat to the inflection point. The slope of the wall at the inflection point can be chosen arbitrarily, up to a maximum value of one-half of the Prandtl-Meyer angle corresponding to the chosen exit Mach number, $v_{\mathrm{e}}$.

Because of the source-flow approximation in the Foelsch method, the flow quality of the computed contours was checked by performing computations with the SAIC SCHNOZ code (ref. 22). For internal-flow computations, the SCHNOZ code solves the Euler equations in a spatially marching fashion. The $\mathrm{SCHNOZ}$ calculations were started in the nozzles at a Mach number of 1.01 and the corresponding area ratio of 1.000083 . The Mach 1.01 contour was assumed to be straight. A grid of 41 mesh points in the cross-stream direction was used; the SCHNOZ code controls the streamwise mesh spacing, which was relatively fine near the throat and became coarser with increasing downstream distance. The total number of streamwise stations ranged from 260 to 511 .

A series of numerical experiments showed that the uniformity of the flow at the nozzle exit increased with decreasing values of the design parameter $\theta_{i} / v_{e}$, the ratio of the nozzle slope at the inflection point to the Prandtl-Meyer angle. The indicated values were chosen based on a compromise between nozzle flow uniformity and nozzle length (increasing the nozzle length increases the boundary-layer growth, particularly in the supersonic region).

Nozzles with nominal exit Mach numbers of 1.4, $1.75,2.60$, and 3.40 were designed. These Mach numbers are representative of combustor-exit Mach numbers corresponding to free-stream Mach numbers of 5.3, 7.3, 10.3 , and 14 , respectively-the Mach numbers available (or projected) for operation of the 3.5-Foot Hypersonic Wind Tunnel.

The nozzles were designed to a fixed overall length of $16.180 \mathrm{~cm}$ and an exit height of $2.032 \mathrm{~cm}$. Definitions of the lengths of the expansion section, the contraction, and the contraction extension, as well as the coordinate systems $x_{n}, y_{n}$ and $x_{p}, y_{p}$, are given in figure 41 .

Contraction extensions were used for the Mach 1.4 and 1.75 nozzles. Since the area ratios corresponding to these Mach numbers are so low, nozzles designed for these Mach numbers are sensitive to boundary-layer displacement effects. The contraction extension was used to accommodate the constraints imposed by the nozzle block design and to minimize the length of the supersonic portion of the nozzle. The value of approximately $2.64 \mathrm{~cm}$ for the height of the constant-area duct was chosen to provide a minimum thickness of $0.64 \mathrm{~cm}$ of nozzle-block material in the contraction region. The inlet portion of the contraction extension was chosen to be a circle-arc tangent to the plenum chamber and to the straight section of the contraction extension.

Boundary-layer calculations were performed for each of the nozzle contours, using the 2-D finitedifference code of Cebeci et al. (ref. 20). Pressure distributions were obtained from the SCHNOZ code in the supersonic region, and 1-D computations were used to estimate the pressure distribution upstream of the throat. The boundary layer computations were started at the upstream corner of the nozzle block, and the boundary layer was assumed to be turbulent. The reservoir conditions used to compute the Reynolds number were chosen to be representative of experimental conditions. Displacement-thickness distributions were computed for both the curved and straight (cowl side) nozzle walls, summed, and subtracted from the curved surface in the direction normal to the original surface. Since this resulted in a contour with an exit height greater than the target design value, the nozzles were then rescaled with an increased contraction length, in order to maintain a fixed overall length. Linear scaling was used for the inviscid region, and the boundary layer growth was assumed to be proportional to the 0.8 power of the axial dimension. A complete recalculation was performed for the Mach 2.6 and 3.4 nozzles. A check of the scaling procedure with a repeated calculation indicated a maximum error of 0.4 percent in displacement thickness.

An end-to-end check of the Mach 1.75 nozzle was performed prior to its construction with the 2-D, Reynolds-averaged, Navier-Stokes Code FANSI (ref. 23). Results of this calculation showed that the primary source of nonuniformity in the nozzle-exit plane flow was introduced by the contraction. A velocity gradient existed at the geometric throat such that the boundary-layer edge velocity at the curved surface was approximately 10 per cent greater than the corresponding velocity along the straight surface. This nonuniformity was reduced to 1 percent in the subsequent supersonic nozzle expansion.

Tabulated nozzle data are included in the data files associated with this study. These tabulations include nozzle profile coordinates in the $x_{p}, y_{p}$ system (see fig. 41); the values DC1 and DS1, the displacement thickness distributions computed for the curved and straight surfaces, respectively; the sum of the thicknesses 
resolved in the direction normal to the surface, DT; and the first and second differences of the corrected ordinates, FP and FPP.
Figure 42 shows the final nozzle contours and the computed displacement-thickness distributions for $\mathrm{M}_{\mathrm{j}}=1.40,1.74,2.60$, and 3.40. 


\section{REFERENCES}

1. Marvin, J. G.: CFD Validation Experiments for Hypersonic Flows. AIAA Paper 92-4024, July 1992.

2. Ruffin, S. M.; Venkatapathy, E.; Keener, E. R.; and Nagaraj, N.: Computational Design Aspects of a NASP Nozzle/Afterbody Experiment. AIAA Paper 89-0446, Jan. 1989.

3. Ruffin, S. M.; Venkatapathy, E.; Keener, E. R.; Spaid, F. W.; and Lee, S. H.: Single Expansion Ramp Nozzle Simulations. AIAA Paper 92-0387, Jan. 1992.

4. Spaid, F. W.; and Keener, E. R.: Experimental Results for a Hypersonic Nozzle/Afterbody Flow Field, AIAA Paper 92-3915, July 1992.

5. Spaid, F. W.; and Keener, E. R.: Hypersonic Nozzle/Afterbody CFD Code Validation Part I: Experimental Measurements. AIAA Paper 93-0607, Jan. 1993.

6. Hopkins, E. J.; Keener, E. R.; and Louie, P. T.: Direct Measurements of Turbulent Skin Friction on a Nonadiabatic Flat Plate at Mach Number 6.5 and Comparisons With Eight Theories. NASA TN D-5675, Feb. 1970.

7. Keener, E. R.; and Hopkins, Edward J.: Turbulent Boundary-Layer Velocity Profiles on a Nonadiabatic Flat Plate at Mach Number 6.5. NASA TN D-6907, Aug. 1972.

8. Clauser, F. H.: Turbulent Boundary Layers in Adverse Pressure Gradients. J. Aeronaut. Sci., vol. 21, Feb. 1954, pp. 91-108.

9. Hopkins, E. J.; and Keener, E. R.: Study of Surface Pitots for Measuring Turbulent Skin Friction at Supersonic Mach Numbers-Adiabatic Wall. NASA TN D-3478, July 1966.

10. Keener, E. R.; and Hopkins, E. J.: Use of Preston Tubes for Measuring Hypersonic Turbulent Skin Friction. NASA TN D-5544, Nov. 1969.

11. Kussoy, M. I.; Horstman, C. C.; and Acharya, M.: An Experimental Documentation of Pressure Gradient and Reynolds Number Effects on Compressible Turbulent Boundary Layers. NASA TM-78488, June 1978.
12. Ames Research Staff: Equations, Tables, and Charts for Compressible Flow. NACA Rept. 1135 , 1953.

13. Bertram, M. H.: Comment on "Viscosity of Air." J. Spacecraft and Rockets, vol. 4, no. 2, Feb. 1967, pp. 287-288.

14. Dudzinski, T. J.; and Krause, L. N.: Flow Direction Measurement with Fixed-Position Probes. NASA TM X-1904, 1969.

15. Vas, I. E.: Flowfield Measurements Using a Total Temperature Probe at Hypersonic Speeds. AIAA J., vol. 10, no. 3, March 1972, pp. 317-323.

16. Cubbage, J. M.; Talcott, N. A., Jr.; and Hunt, J. L.: Scramjet Exhaust Simulation Technique for Hypersonic Aircraft Nozzle Design and Aerodynamic Tests. AIAA Paper 77-82, Jan. 1977.

17. Oman, R. A.; Foreman, K. M.; Leng, J.; and Hopkins, H. B.: Simulation of Hypersonic Scramjet Exhaust, NASA CR-2494, March 1975.

18. Pittman, J. L.: A Mach 6 External Nozzle Experiment with Argon-Freon Exhaust Simulation. SAE Paper 892315, Sept. 1989.

19. Van Driest, E. R.: Turbulent Boundary Layer in Compressible Fluids. J. Aeronaut. Soc., vol. 18, no. 3, March 1951, pp. 145-160.

20. Cebeci, T.; and Smith, A. M. O.: Analysis of Turbulent Boundary Layers. Academic Press, New York, 1974, p. 258.

21. Foelsch, K.: A New Method of Designing TwoDimensional Laval Nozzles for a Parallel and Uniform Jet. North American Aviation Report NA-46-235, March 1946.

22. Wolf, D. E.: Overview of Hypersonic Scramjet Nozzle Code SCHNOZ. Science Applications International Corporation, SAIC/PR TP-91, 1986.

23. Bush, R. H.; Vogel, P. G.; Norby, W. P.; and Haeffele, B. A.: Two-Dimensional Numerical Analysis for Inlets at Subsonic Through Hypersonic Speeds. AIAA Paper 87-1751, June 1987. 
Table 1. Baseline test conditions and variations

\begin{tabular}{lccc}
\hline \hline & Nominal values & Variation among runs & Variation during a run \\
\hline $\mathrm{M}_{\infty}$ & 7.33 & \pm 0.025 & \\
$\left(\mathrm{dM}_{\infty}\right) /(\mathrm{dx})$ & $0.177 / \mathrm{m}$ & & \\
$\mathrm{Pt}_{\infty}$ & $6,895 \mathrm{kPa}$ & $\pm 0.2 \%$ & $\pm 0.1 \%$ \\
$\mathrm{~T}_{\mathrm{t}_{\infty}}$ & $828 \mathrm{~K}$ & $\pm 5.8 \%$ & $\pm 1 \%$ \\
$\mathrm{Re}_{\infty}$ & $14 \times 10^{6} / \mathrm{m}$ & & \\
$\mathrm{p}_{\mathrm{tj}}$ & $374 \mathrm{kPa}$ & $\pm 3 \%$ & $\pm 1 \%$ \\
$\mathrm{~T}_{\mathrm{tj}}$ & $272 \mathrm{~K}$ & $\pm 2 \%$ & $\pm 2 \%$ \\
$\mathrm{P}_{\mathrm{j} j} / \mathrm{p}_{\infty}$ & 310 & $\pm 3 \%$ & $\pm 1 \%$ \\
$\mathrm{Mj}$ & 1.74 & \pm 0.005 & \pm 0.005 \\
\hline \hline
\end{tabular}

Table 2. Boundary-layer rake data summary

\begin{tabular}{ccccccc}
\hline \hline $\mathrm{P}_{\mathrm{tj}} / \mathrm{P}_{\infty}$ & $\delta^{*}$ & $\theta$ & $\mathrm{Re}_{\theta}$ & $\mathrm{H}$ & $\mathrm{C}_{\mathrm{f}}$ & \\
\hline- & 0.367 & 0.0236 & 3,740 & 15.53 & 0.00127 & No. 1-forebody rake \\
- & 0.335 & 0.0244 & 3,533 & 13.71 & 0.00130 & $\mathrm{x}=-36.5$ \\
& & & & & & $\mathrm{y}=-3.96$ \\
312 & 0.178 & 0.0272 & 6,230 & 6.53 & 0.00193 & No. 2-forward ramp rake \\
197 & 0.181 & 0.0278 & 3,853 & 6.49 & 0.00204 & $\mathrm{x}=19.84$ \\
118 & 0.188 & 0.0282 & 2,278 & 6.66 & 0.00248 & $\mathrm{y}=-6.086$ \\
316 & 0.152 & 0.0233 & 5,181 & 6.53 & 0.00202 & \\
307 & 0.164 & 0.0249 & 5,462 & 6.58 & 0.00201 & \\
312 & 0.343 & 0.0366 & 2,470 & 9.40 & 0.00221 & No. 3-aft ramp rake \\
197 & 0.436 & 0.0545 & 2,296 & 7.99 & - & $\mathrm{x}=49.58$ \\
316 & 0.342 & 0.0378 & 1,954 & 9.06 & 0.00241 & $\mathrm{y}=-2.86$ \\
307 & 0.375 & 0.0400 & 1,575 & 9.38 & 0.00227 & \\
\hline \hline
\end{tabular}

Baseline test conditions

Nominal $\mathrm{pt}_{\infty}=6,895 \mathrm{kPa}$

Nominal $\mathrm{T}_{\mathrm{t}_{\infty}}=828 \mathrm{~K}$

Dimensions in $\mathrm{cm}$

Table 3. Preston-tube skin-friction data at baseline test conditions, $x=19.84 \mathrm{~cm}$

\begin{tabular}{ccccc}
\hline \hline $\mathrm{P}_{\mathrm{tj}} / \mathrm{p}_{\infty}$ & $\mathrm{d}$ & $\mathrm{y}$ & $\mathrm{du}_{\tau} / \mathrm{v}_{\mathrm{W}}$ & $\mathrm{C}_{\mathrm{f}}$ \\
\hline 316 & 0.157 & -1.745 & 180 & 0.00130 \\
& 0.239 & -2.380 & 272 & 0.00147 \\
& 0.318 & -3.015 & 363 & 0.00146 \\
307 & 0.157 & -1.745 & 179 & 0.00133 \\
& 0.239 & -2.380 & 272 & 0.00144 \\
& 0.318 & -3.015 & 362 & 0.00137 \\
\hline \hline
\end{tabular}

Dimensions in $\mathrm{cm}$ 


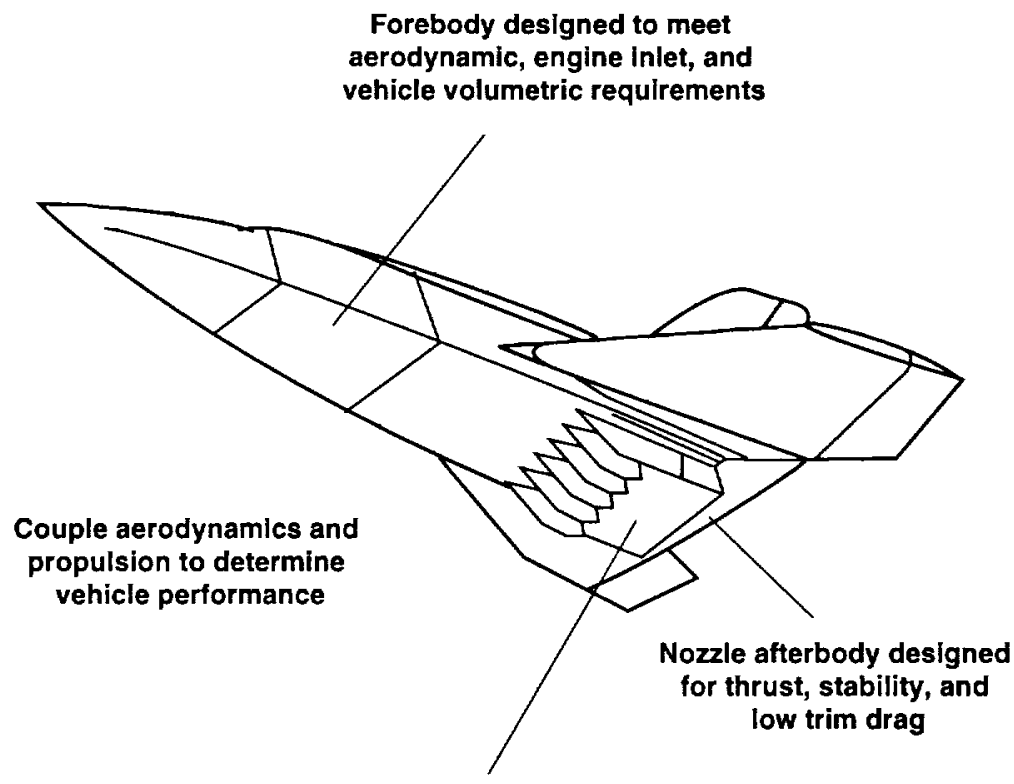

Scramjets sized to meet

mission requirements

Figure 1. Elements of engine-aiframe integration design criteria for hypersonic flight.

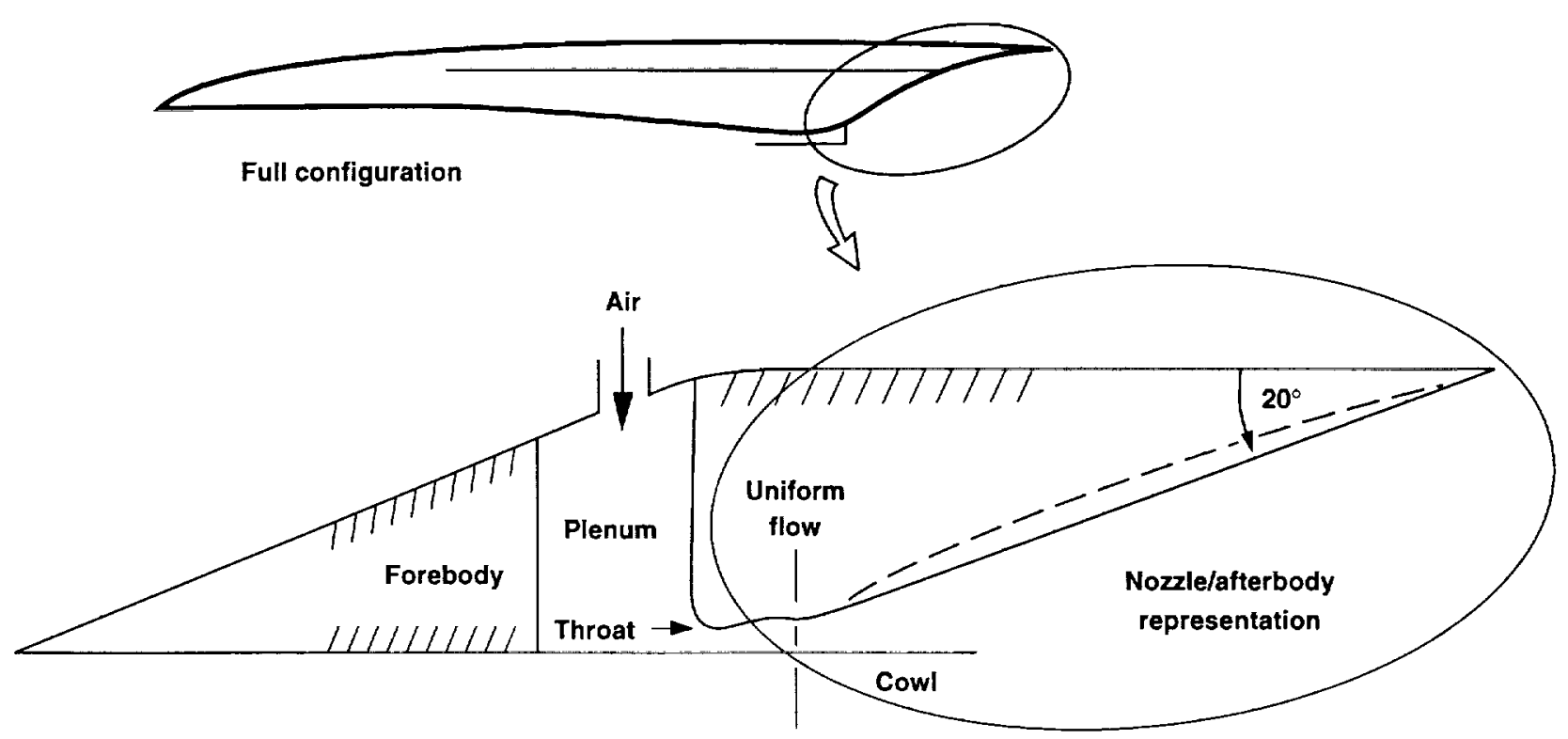

Figure 2. Development of nozzle/afterbody (SERN) model approach. 


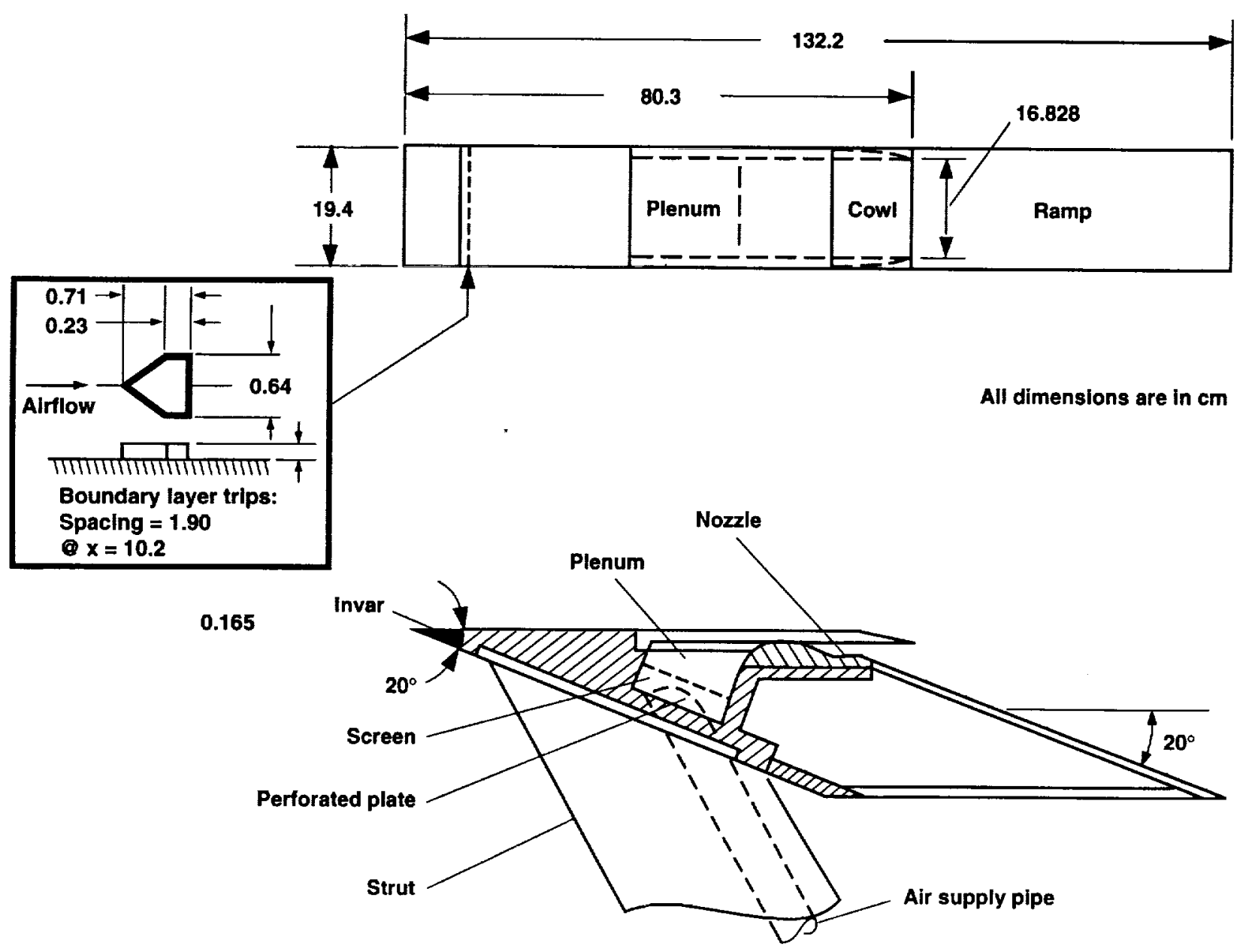

Figure 3. Schematic of hypersonic SERN model. (a) Basic model and boundary-layer trip detail. 


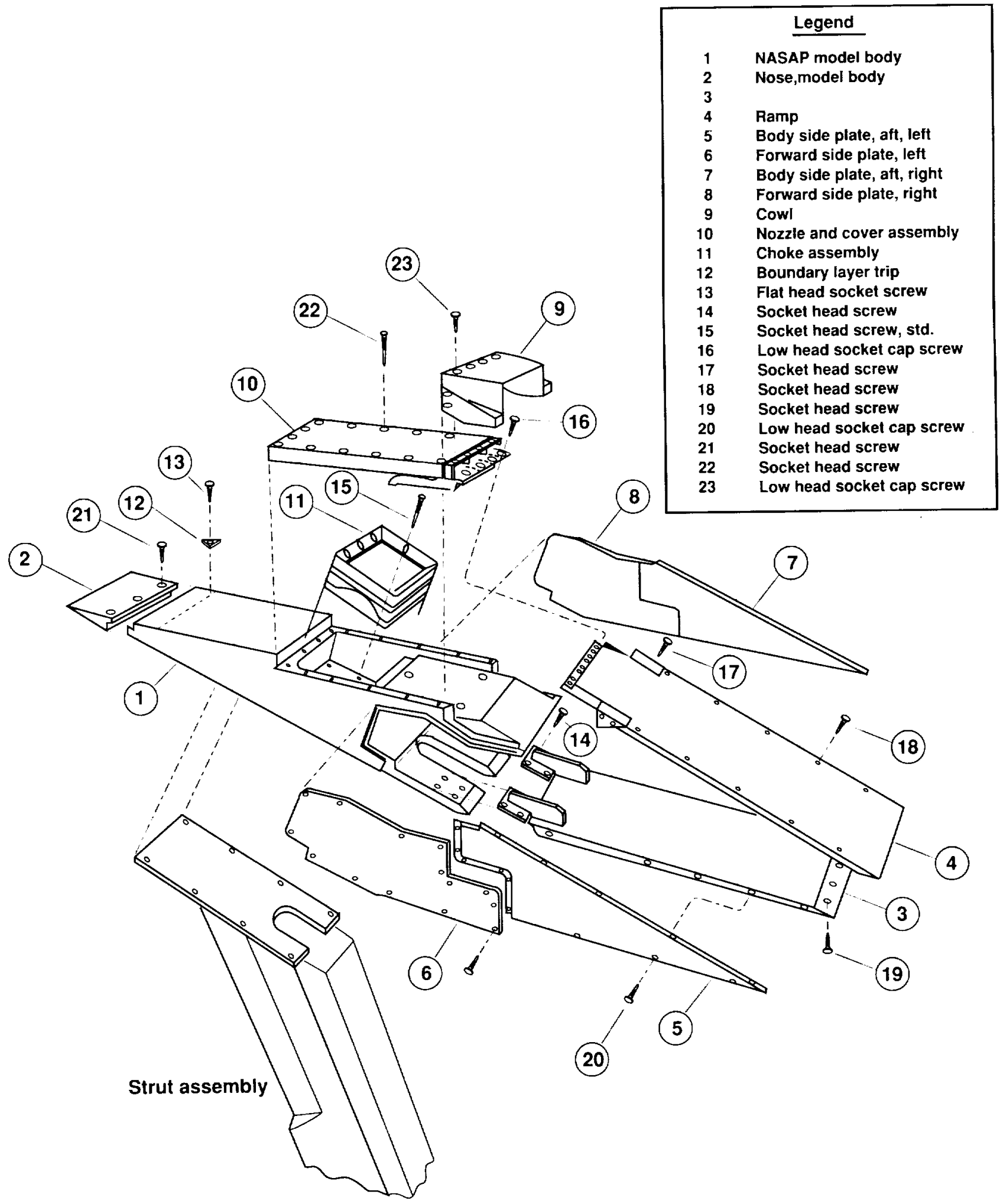

Figure 3. Continued. (b) Exploded view. 


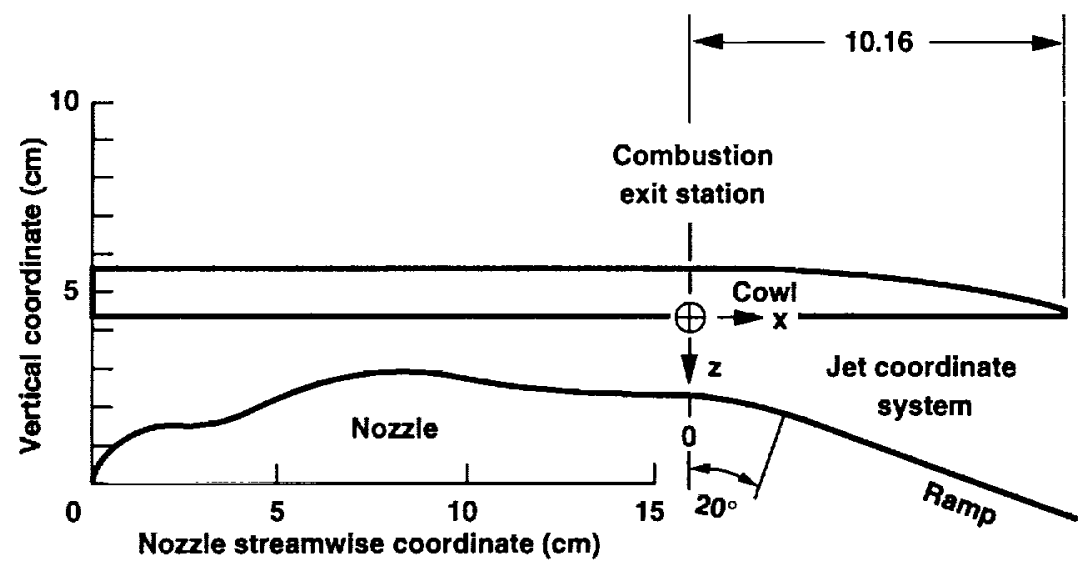

All dimensions are in $\mathrm{cm}$

Figure 3. Continued. (c) Internal nozzle geometry.

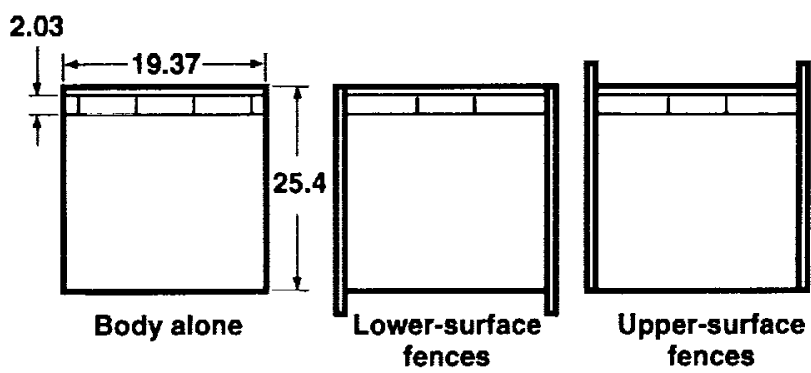

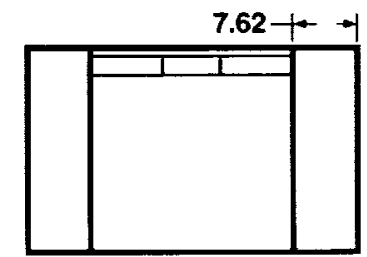

Side extensions

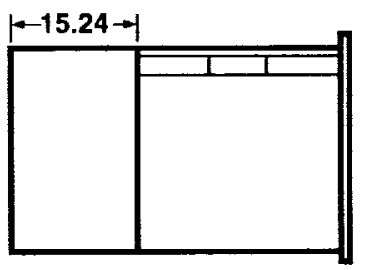

Semispan model; fence and side extension

All dimensions are in $\mathrm{cm}$

Figure 3. Continued. (d) Aternate configuration, rear views. 


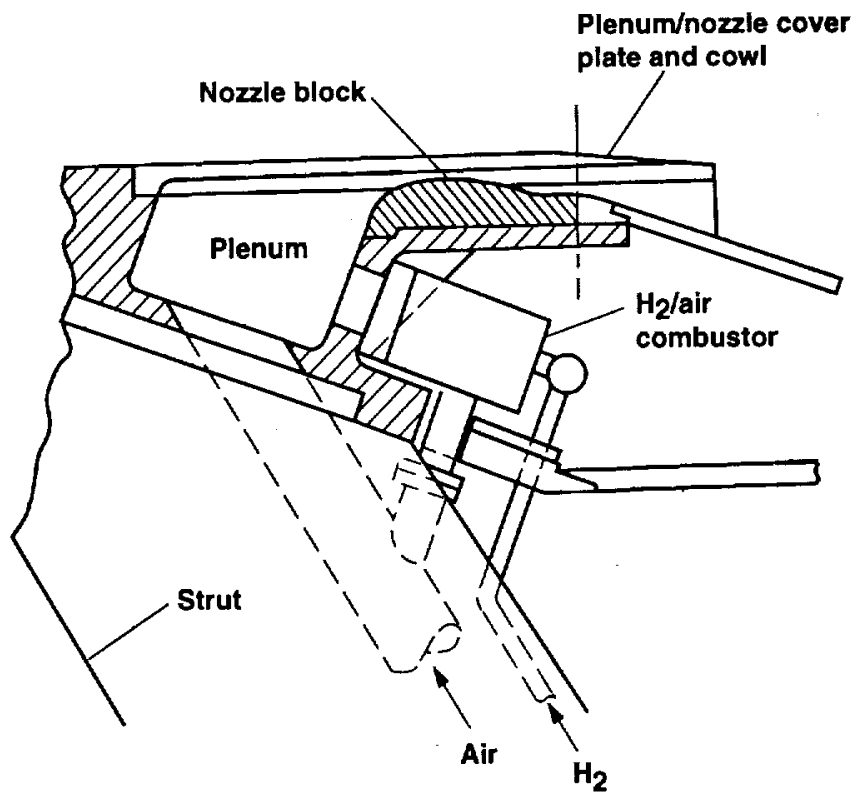

Figure 3. Concluded. (e) Hydrogen/air combustor installation.

29 


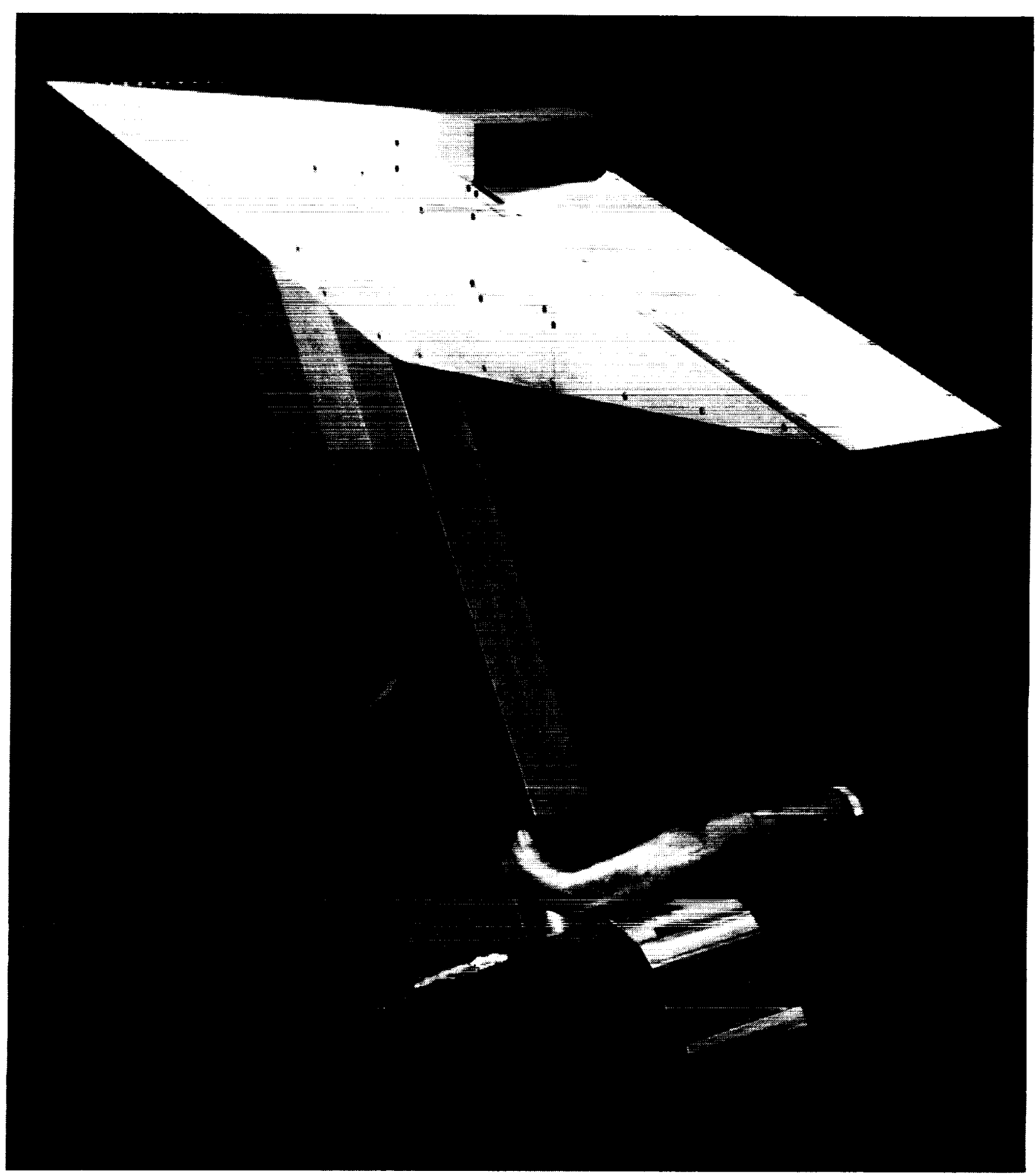

Figure 4. Hypersonic SERN model. (a) 3/4 rear view of model and strut. 


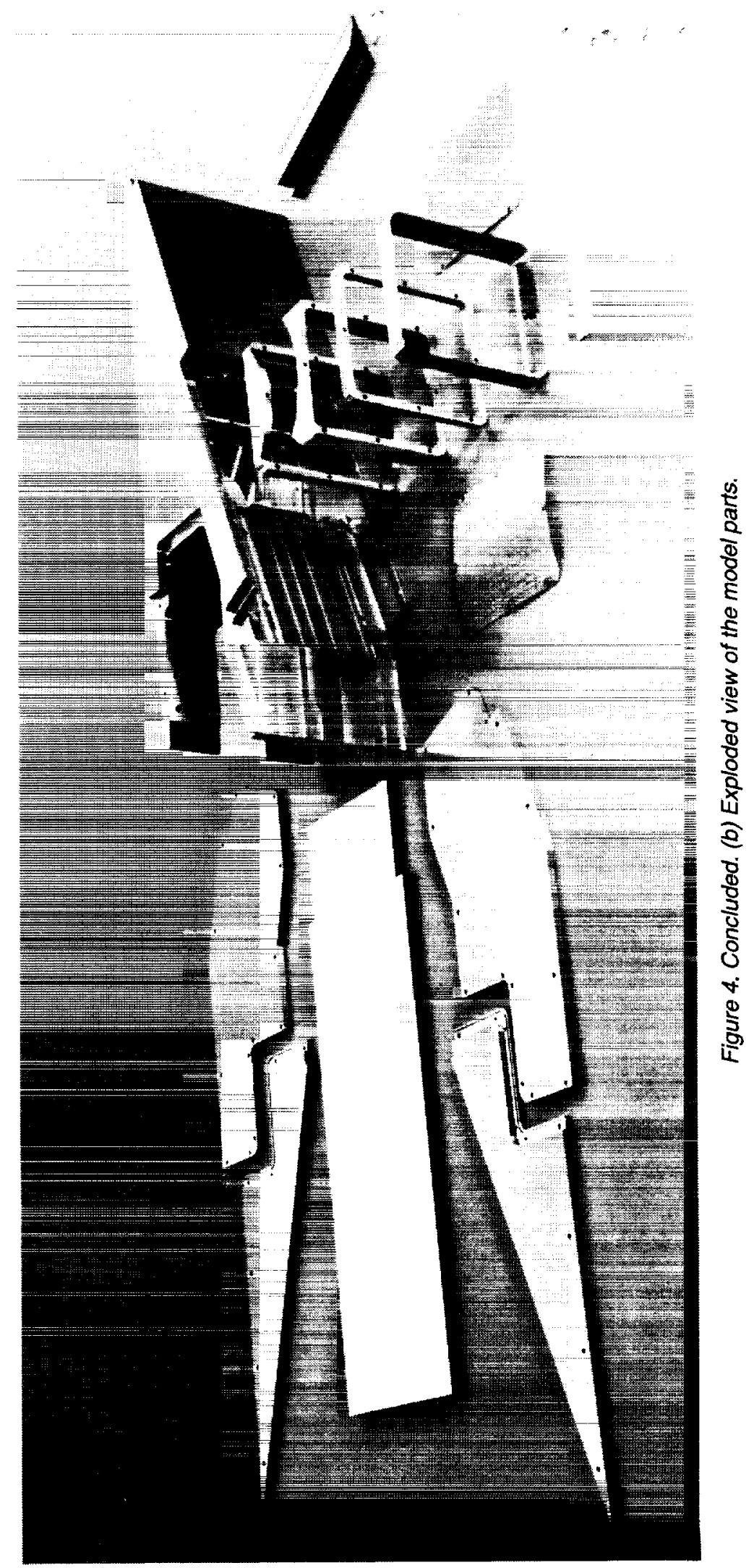




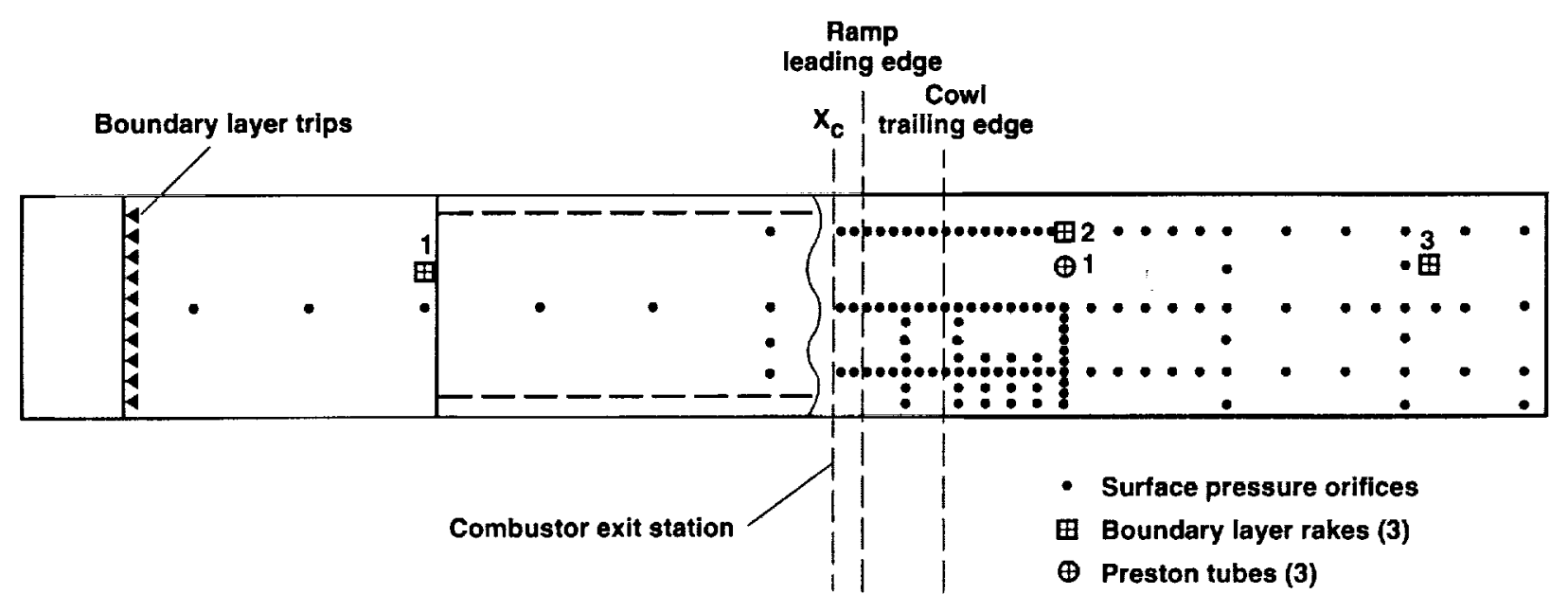

Figure 5. Plan view of model instrumentation locations. 


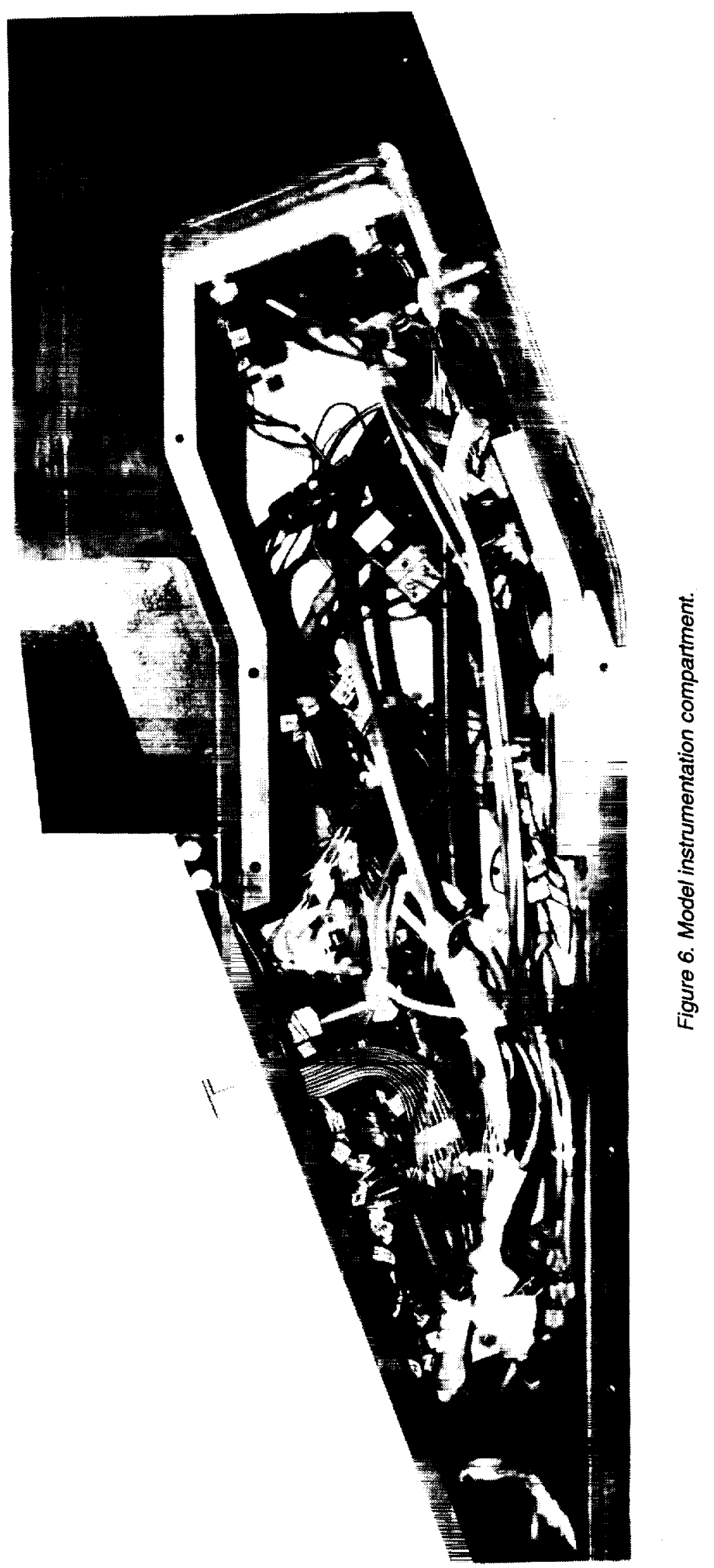


Probe heights $(\mathrm{cm})$

\begin{tabular}{|c|c|r|}
\hline \multicolumn{3}{|c|}{ Aake } \\
\hline 1 & 2 & \multicolumn{1}{c|}{3} \\
\hline 2.540 & 2.540 & 2.540 \\
.000 & .800 & 1.25 \\
.699 & .660 & 1.08 \\
.597 & .546 & .902 \\
.500 & .483 & .742 \\
.394 & .381 & .584 \\
.279 & .254 & .406 \\
.165 & .160 & .267 \\
.058 & .051 & .102 \\
\hline
\end{tabular}

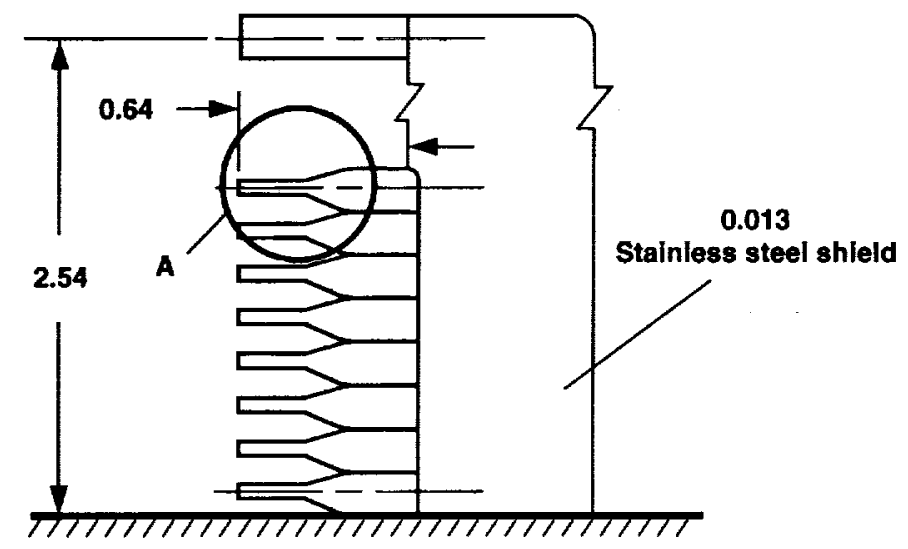

All dimensions are in $\mathrm{cm}$

$0.157 \mathrm{~cm}$ O.D.

(or smaller)

Stainless steel tube

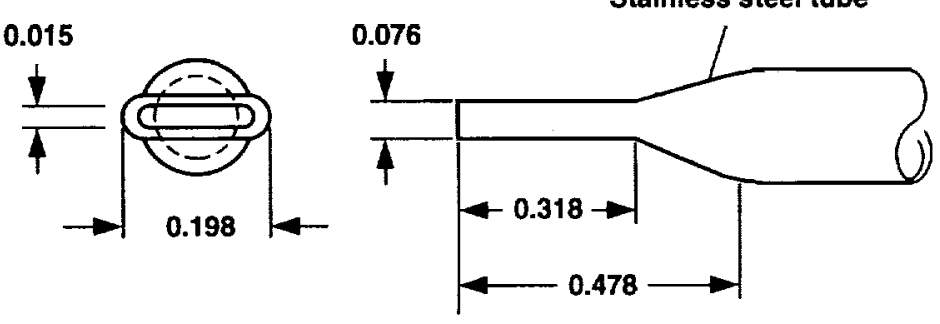

Detail of area "A" above

Figure 7. Dimensions of boundary layer rakes. 


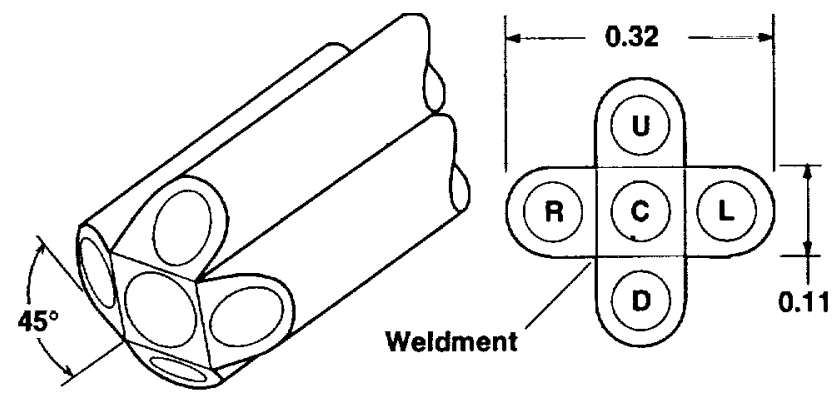

Front View

Dimensions are in $\mathrm{cm}$

Figure 8. Jet-plume traversing-unit probes. (a) Five-hole-probe tip.

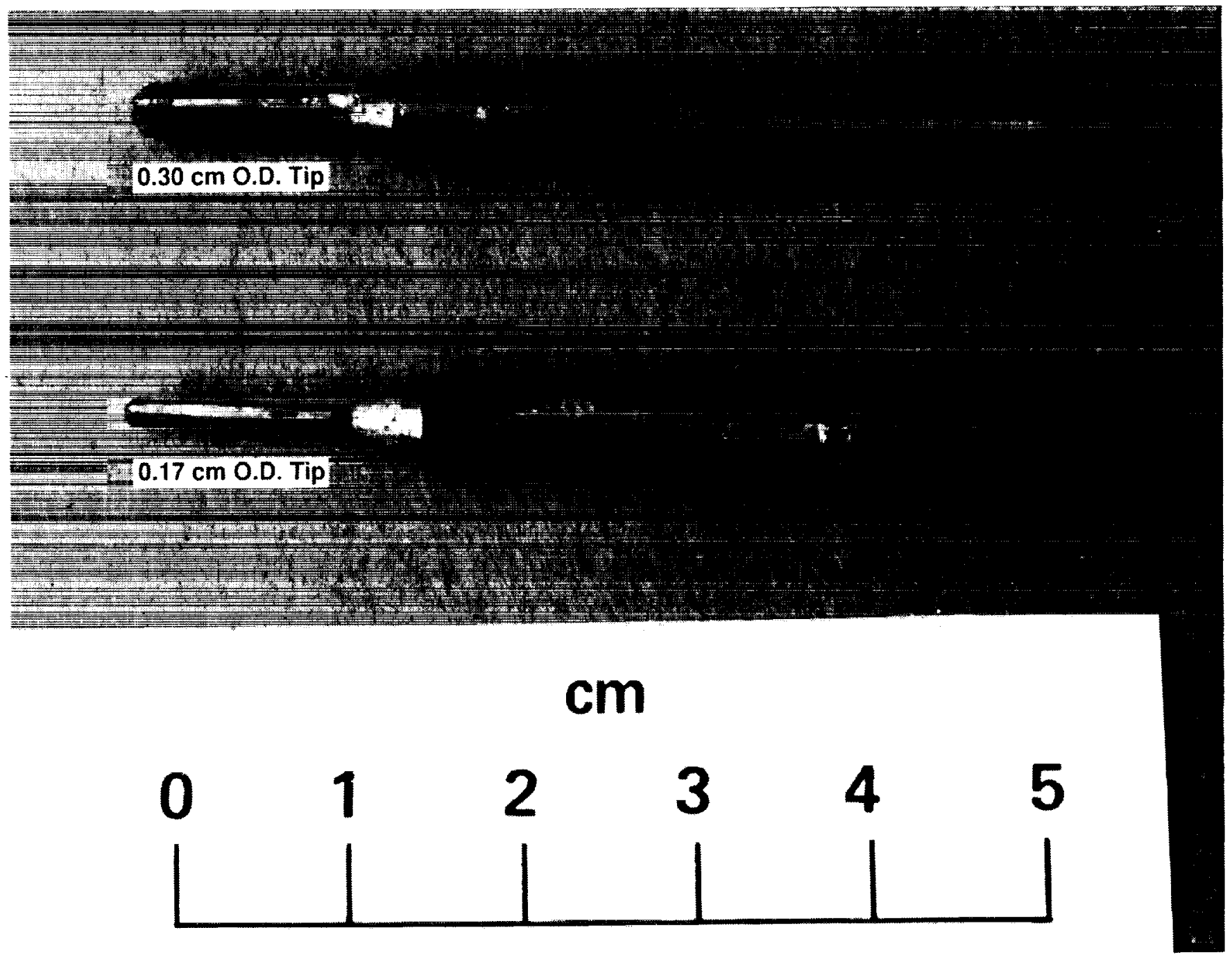

Figure 8. Continued. (b) Five-hole probes, top view. 


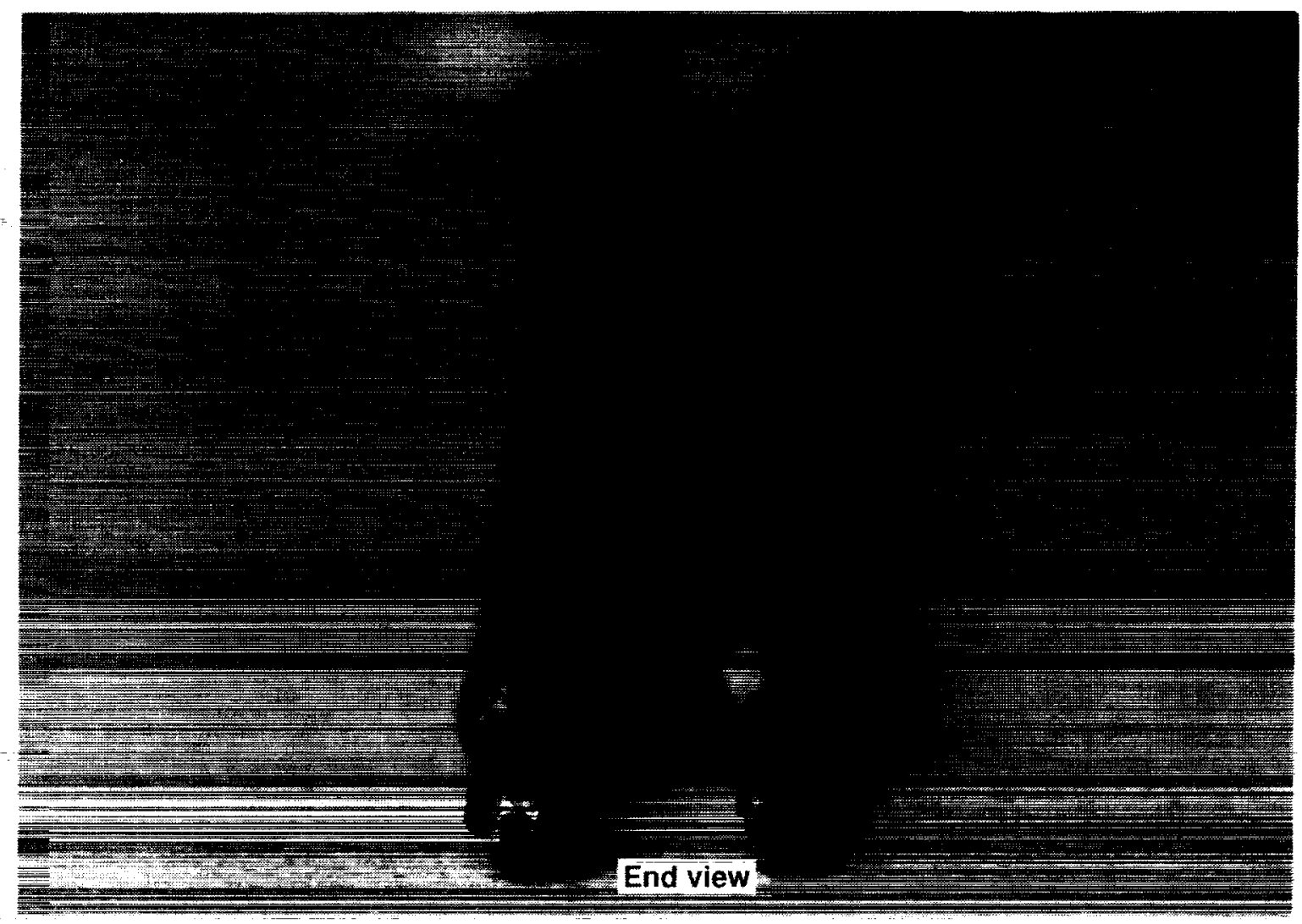

Figure 8. Continued. (c) Five-hole probes, end view.

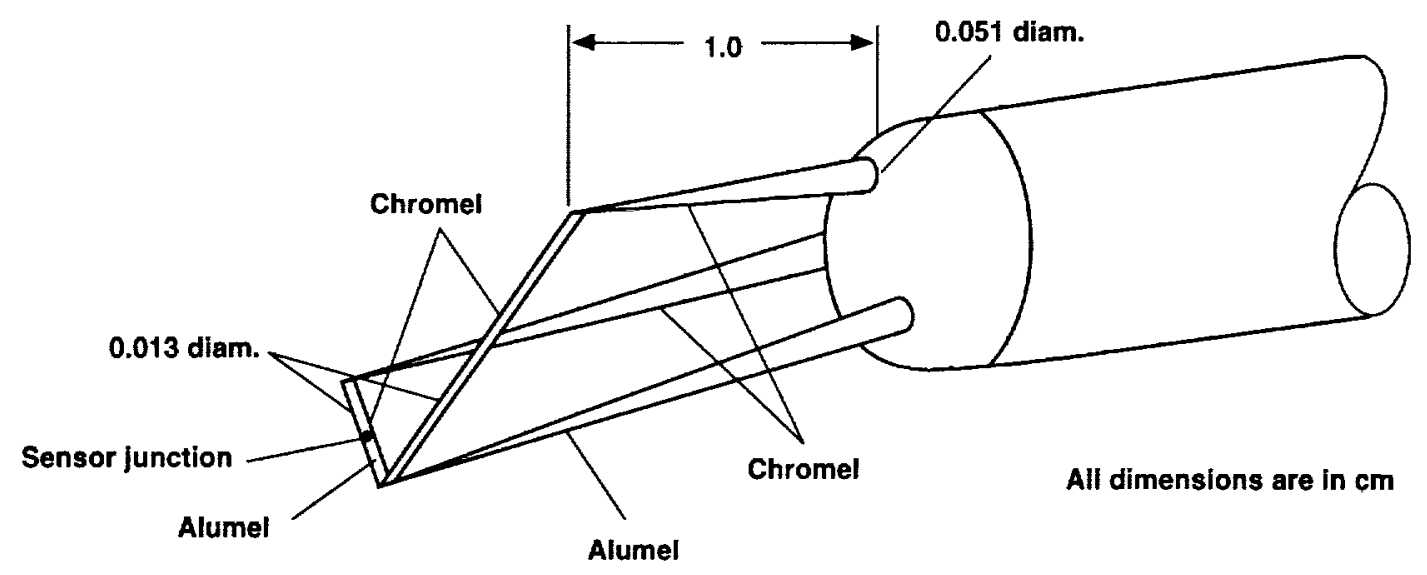

Figure 8. Concluded. (d) Thermocouple probe. 

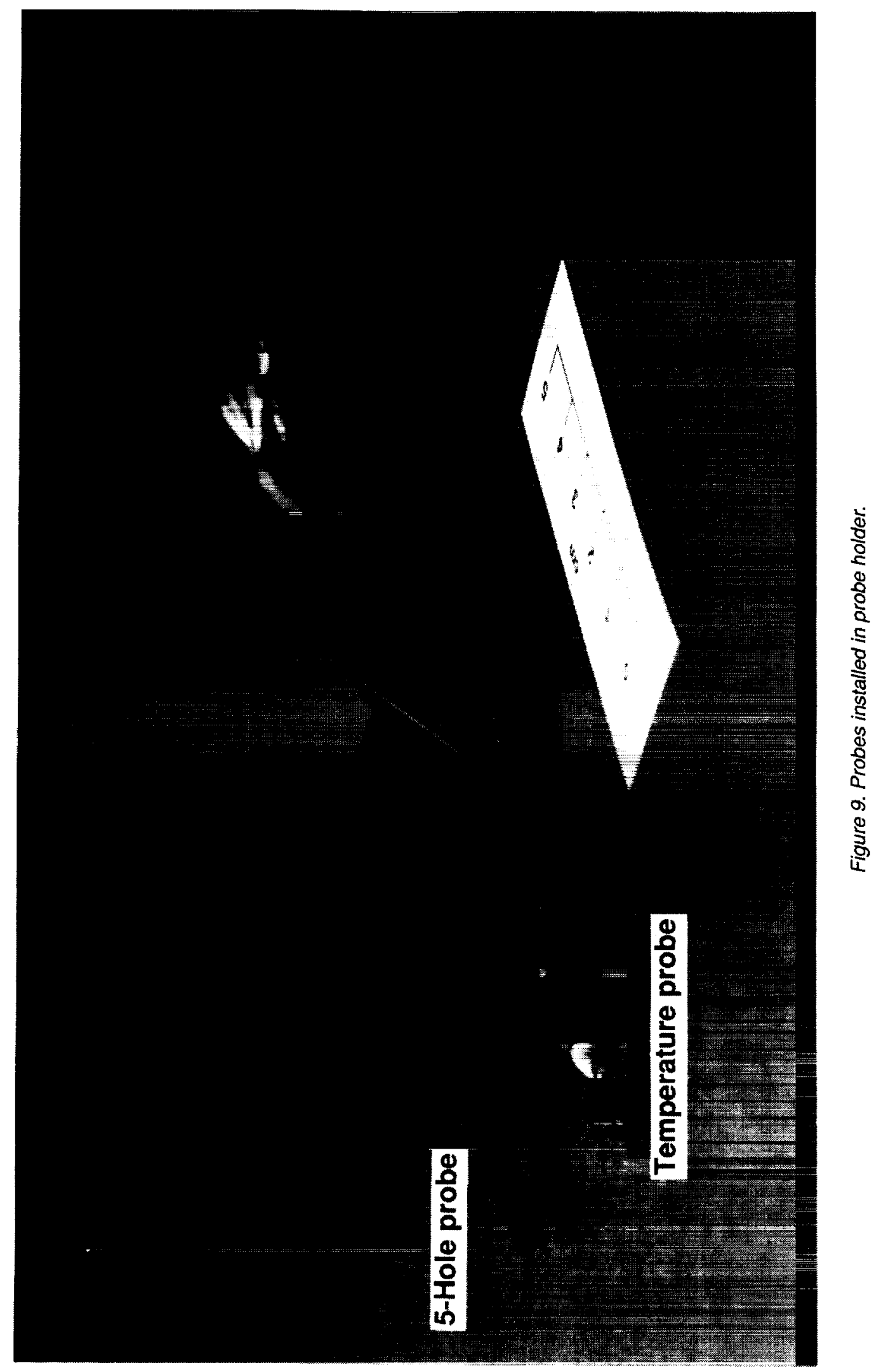


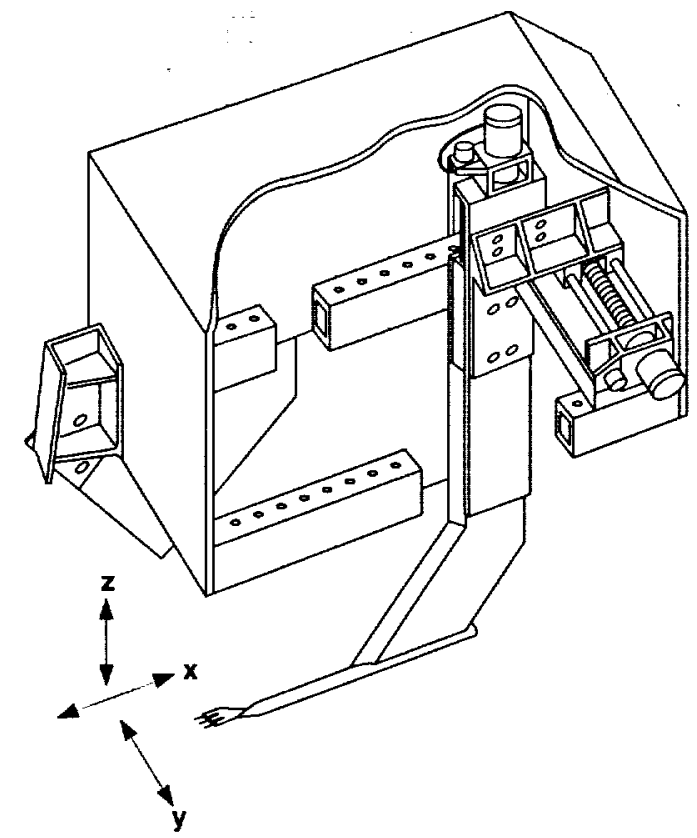

Figure 10. Schematic of jet-plume traversing unit.

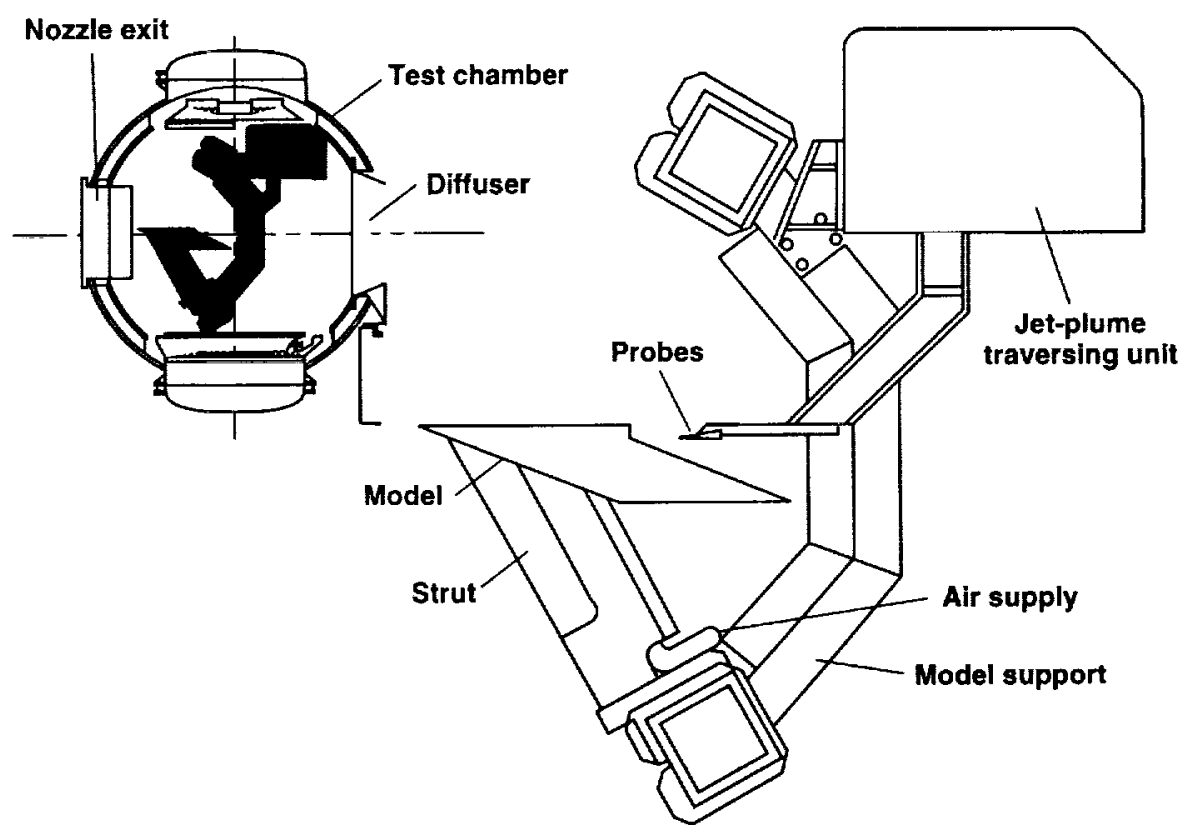

Figure 11. Schematic of model and jet-plume traversing unit installed in the test section of the Ames 3.5-Foot Hypersonic Wind Tunnel. 
ORIGINAL PAGE

BLACK ANO WHITE P'HOTOGRAFr

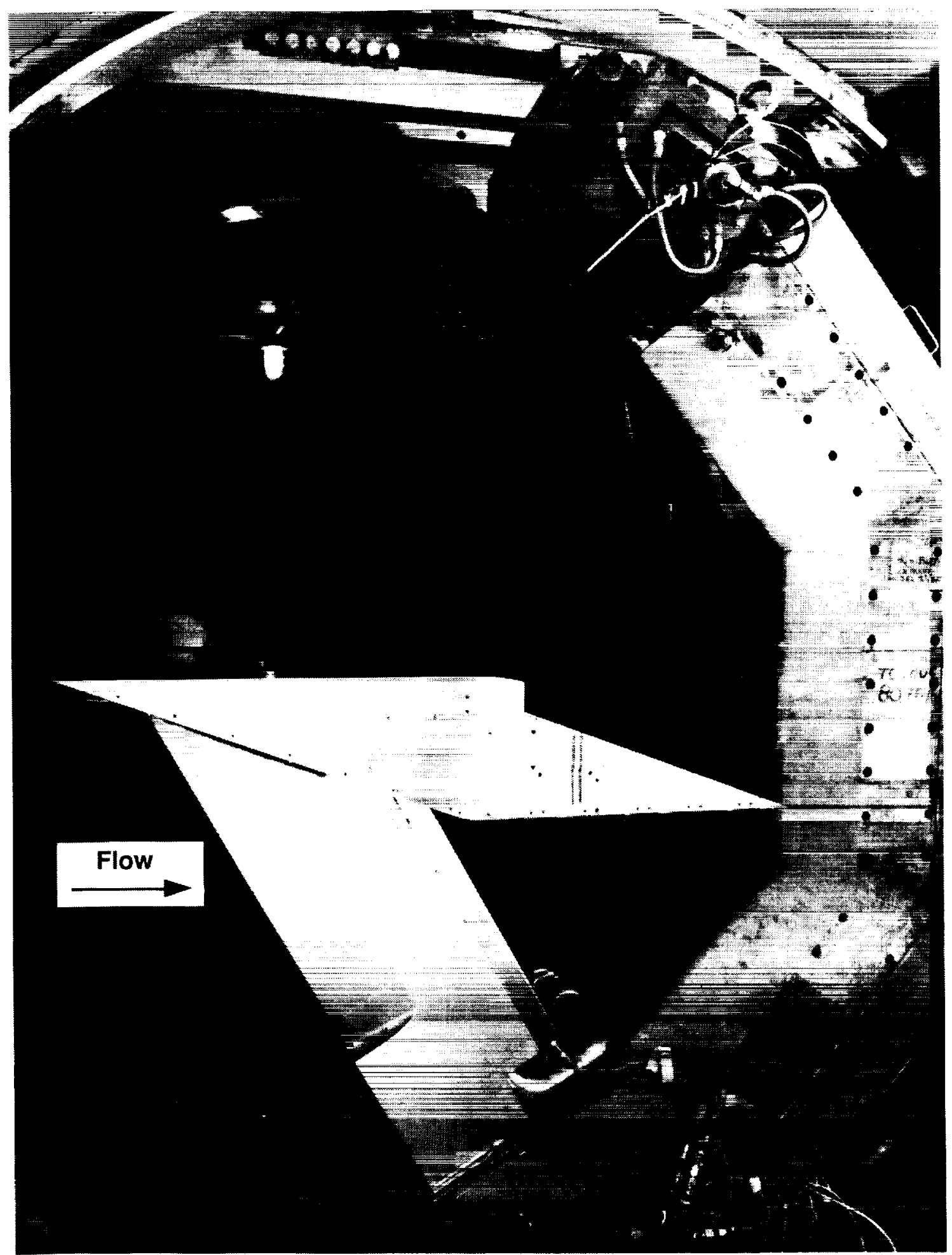

Figure 12. Model-alone installation in the Ames 3.5-Foot Hypersonic Wind Tunnel. (a) Side view. 


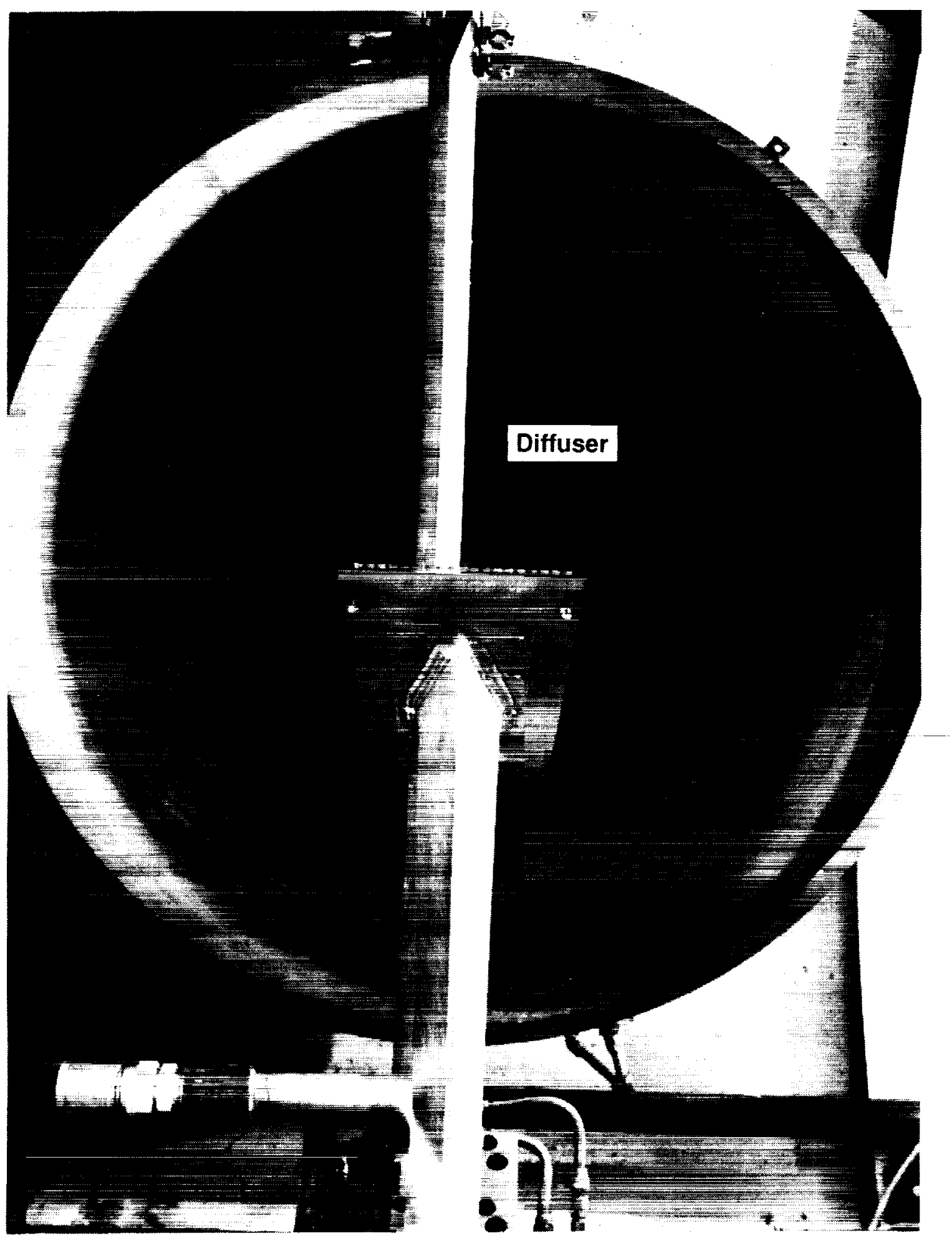

Figure 12. Concluded. (b) Front view. 
OFIGINAL PAGE

BLACK AIIC WHITE PHOTOGRAPH

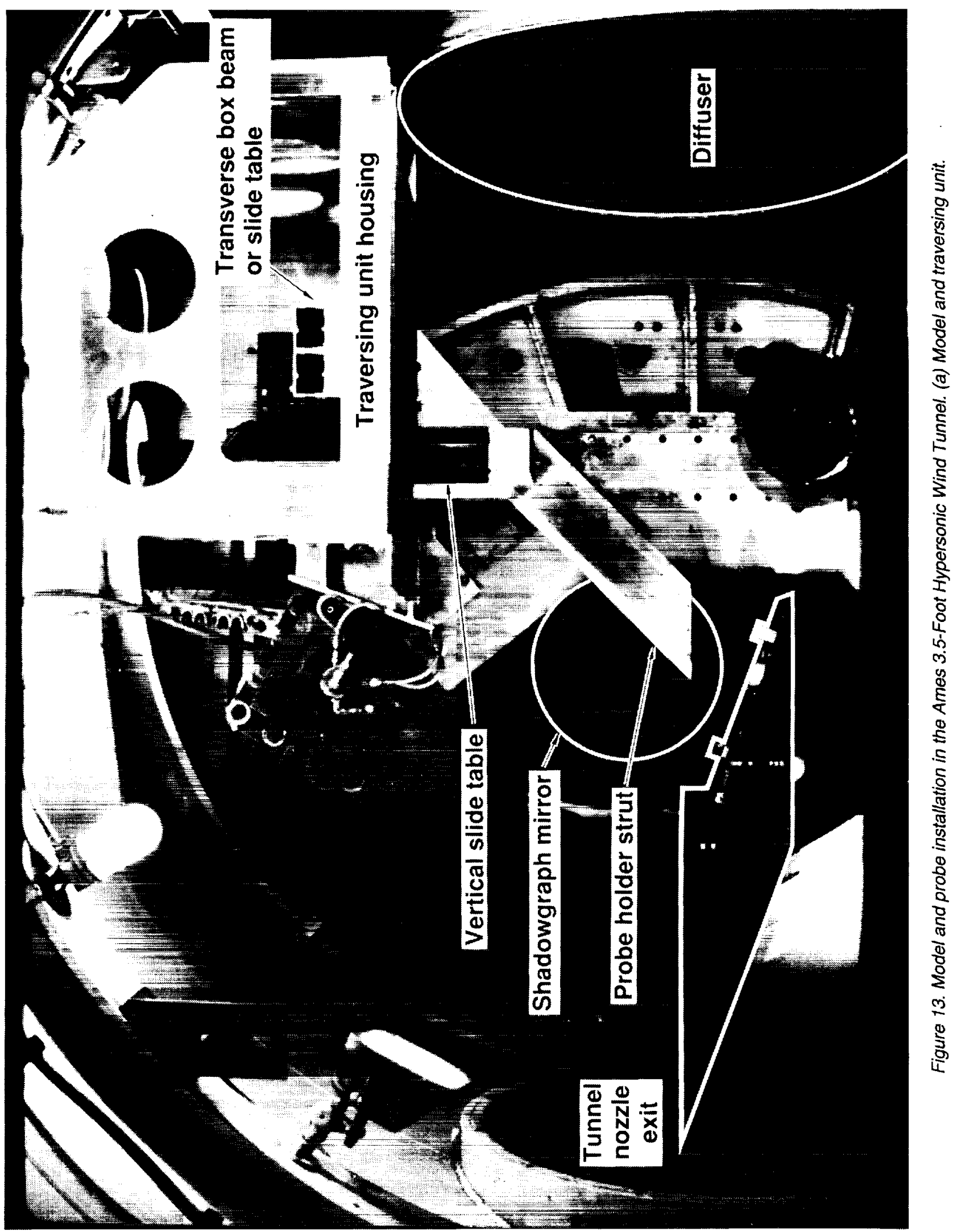




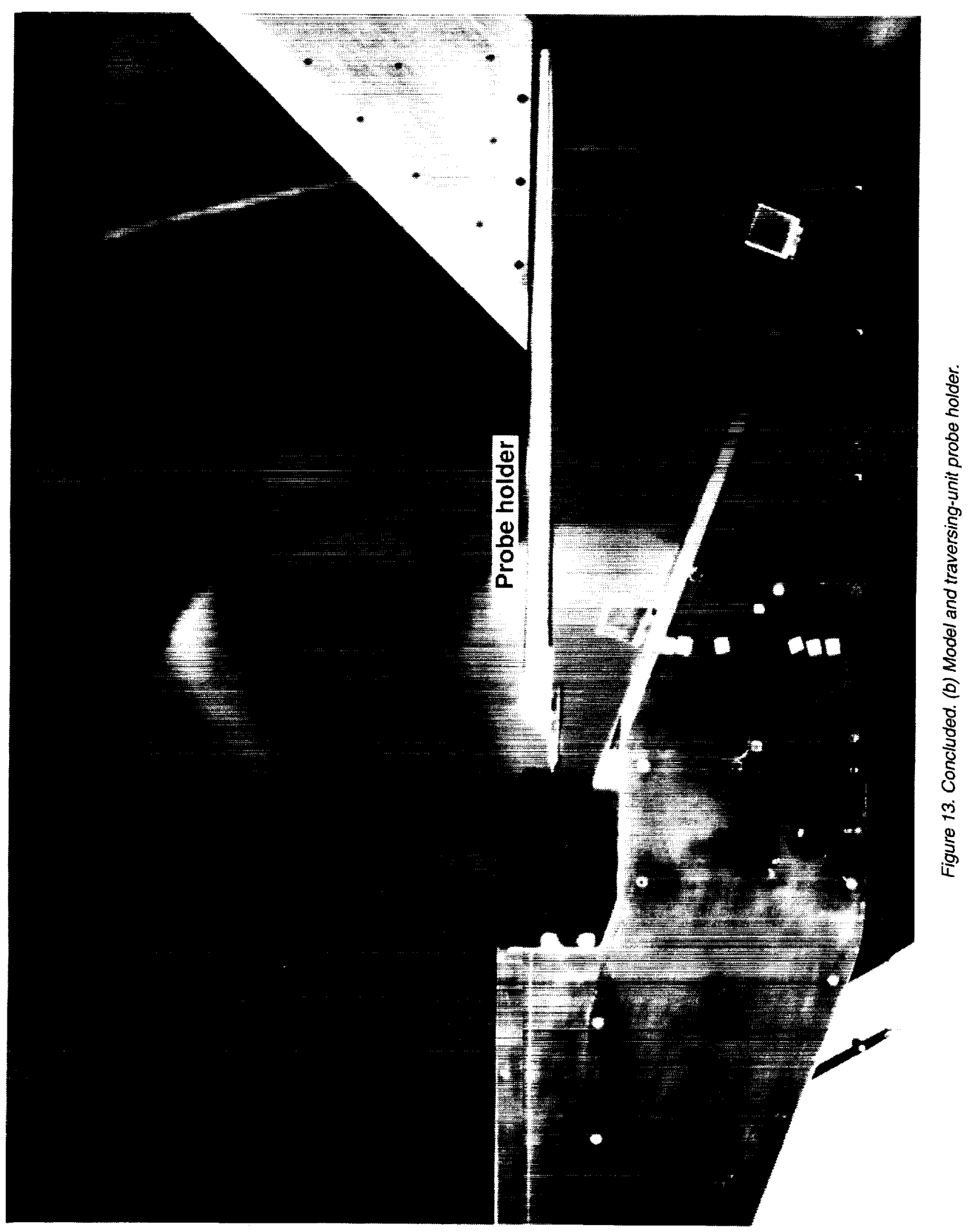


ORTINAL PAGE

BLACK AND WHITS PHOTOGRAPH

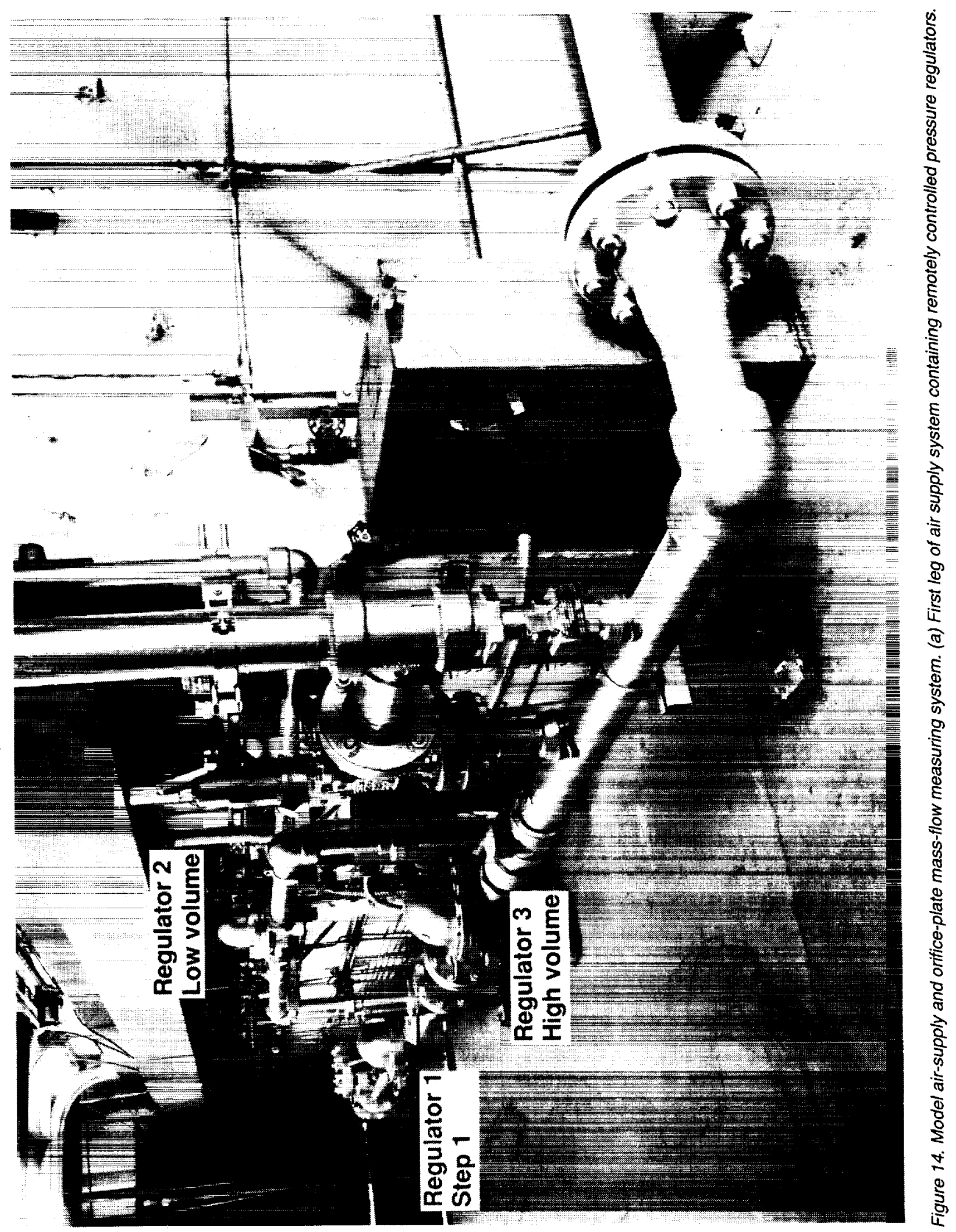




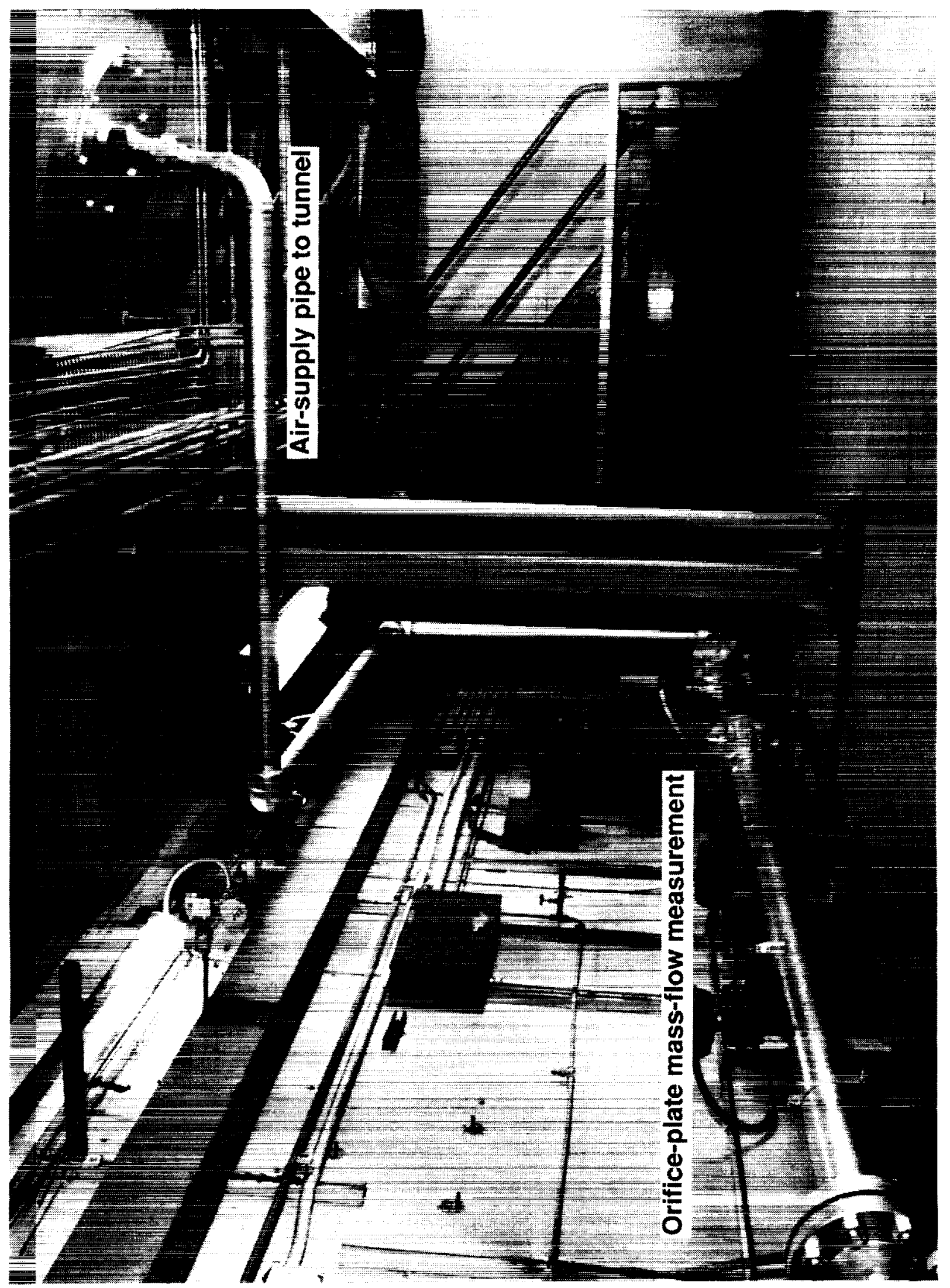

造 


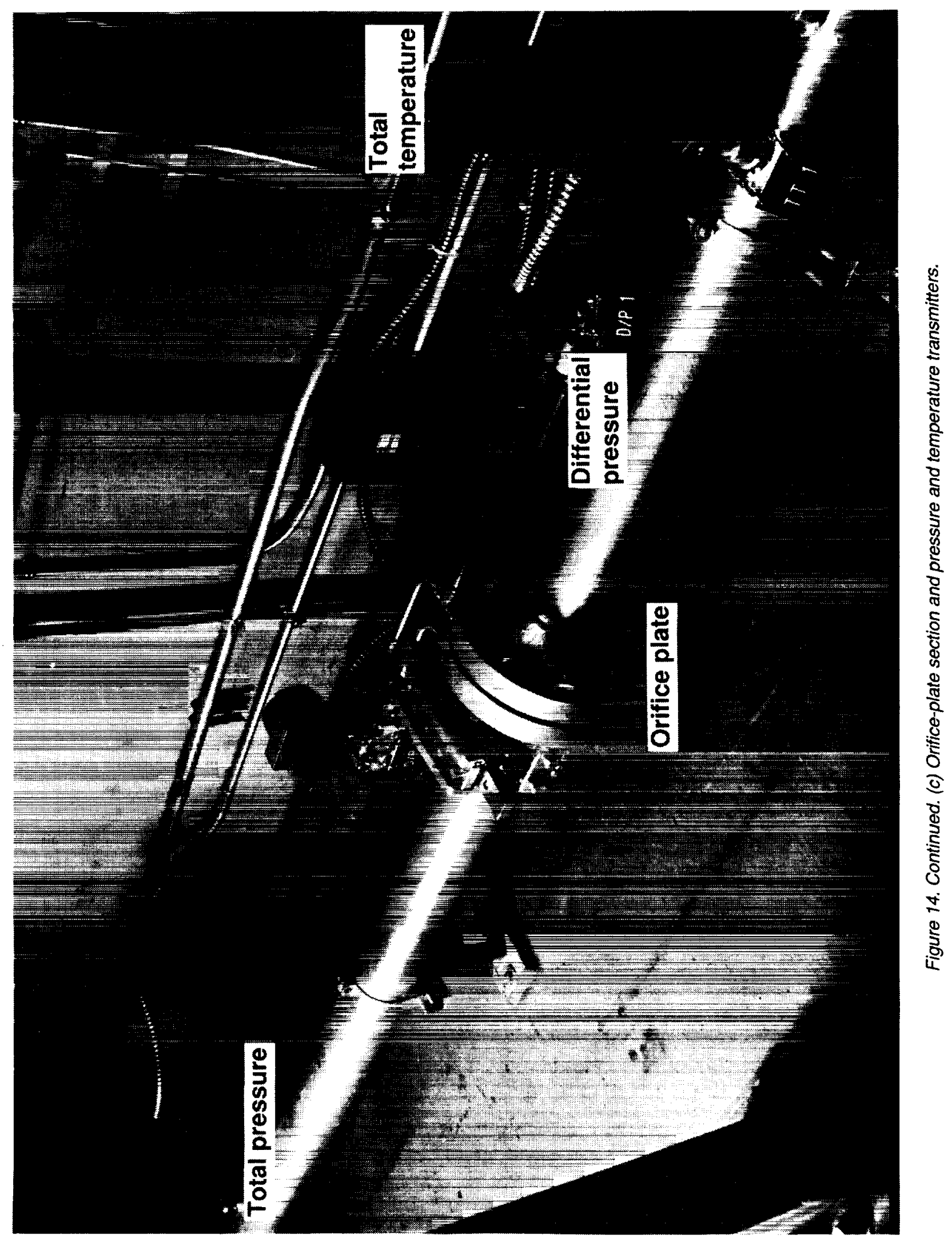

GRIONAL PAOE

BLACK AND WHITE PHOTOGRAPH 


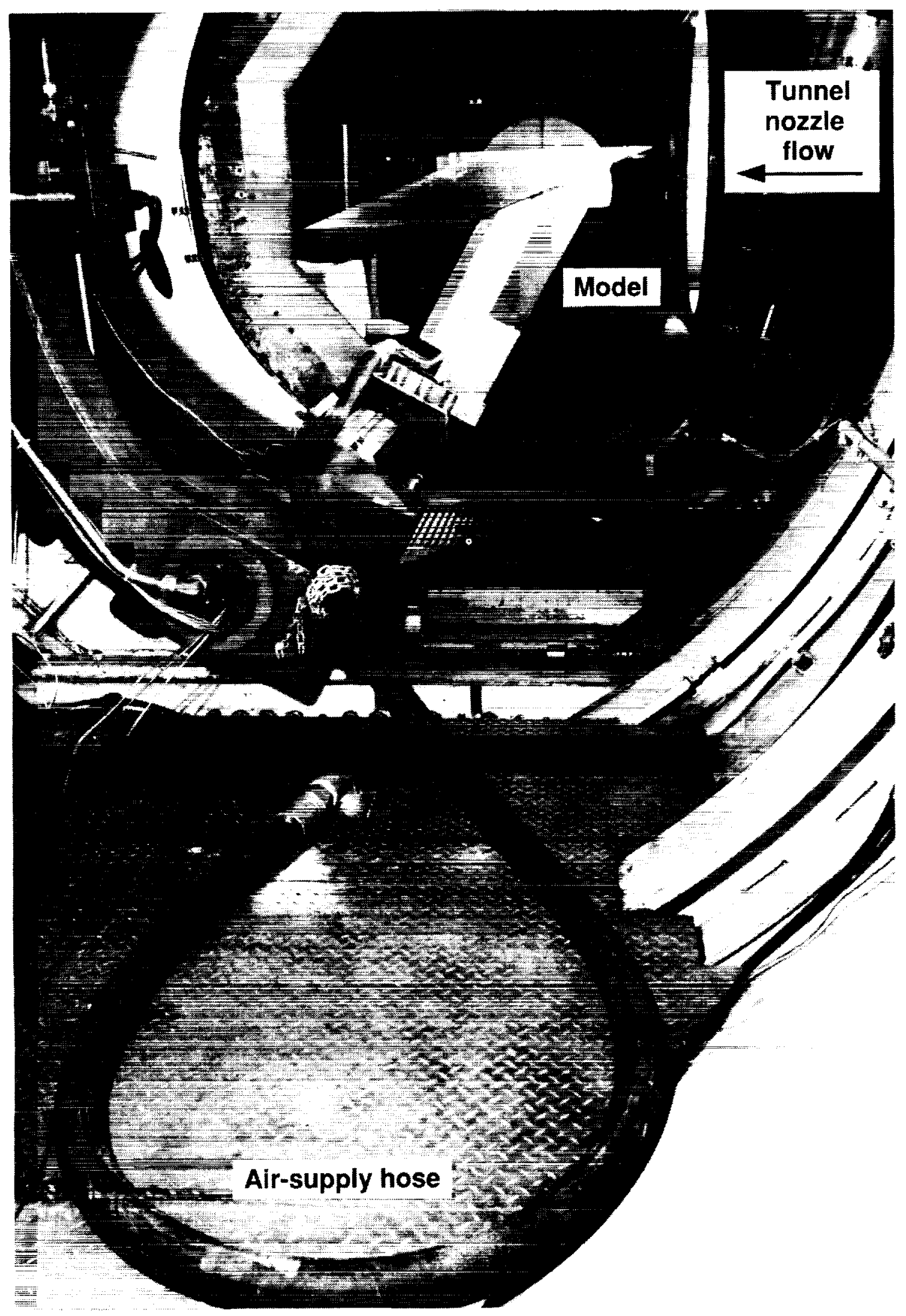

Figure 14. Concluded. (d) Model and air supply pipe. 


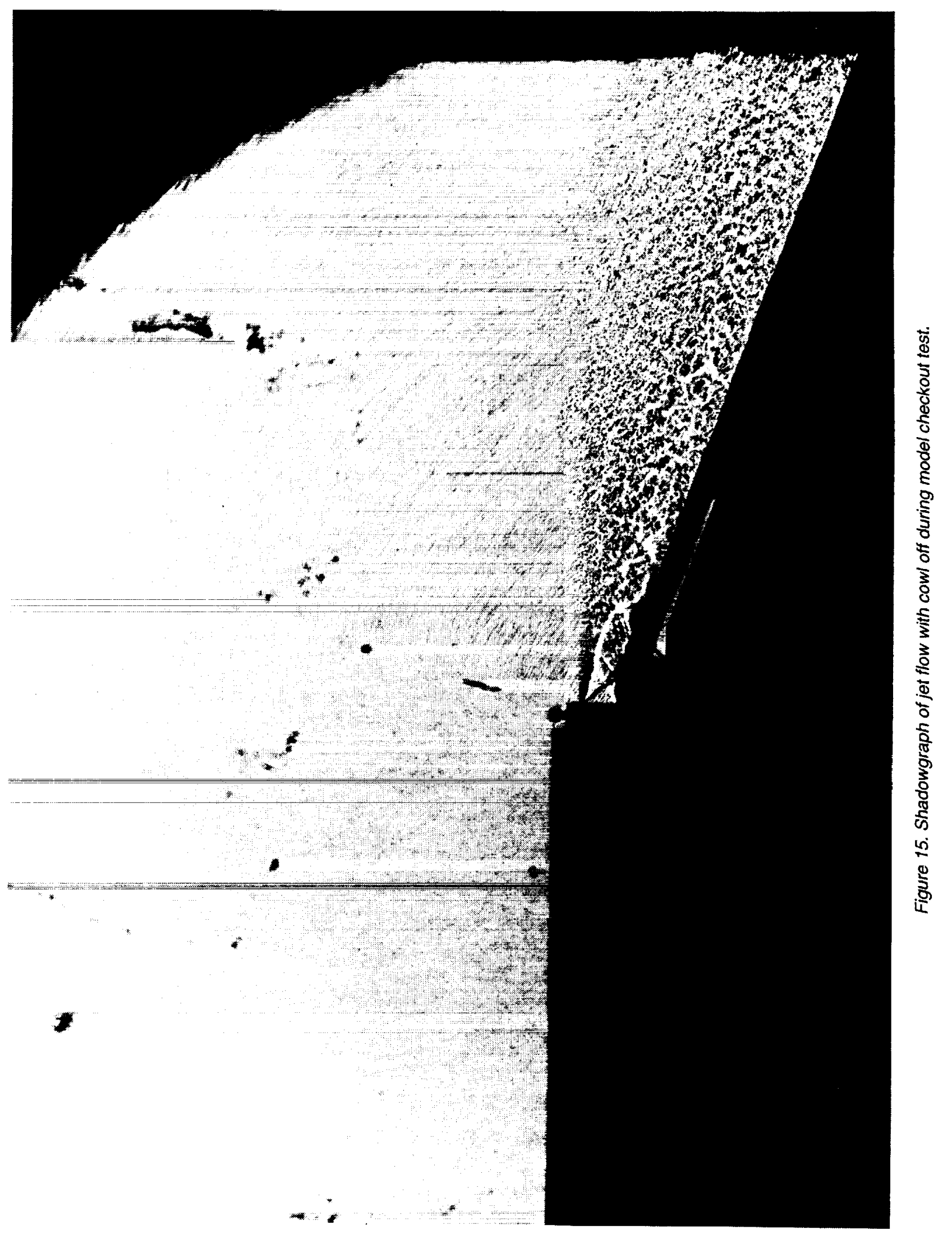




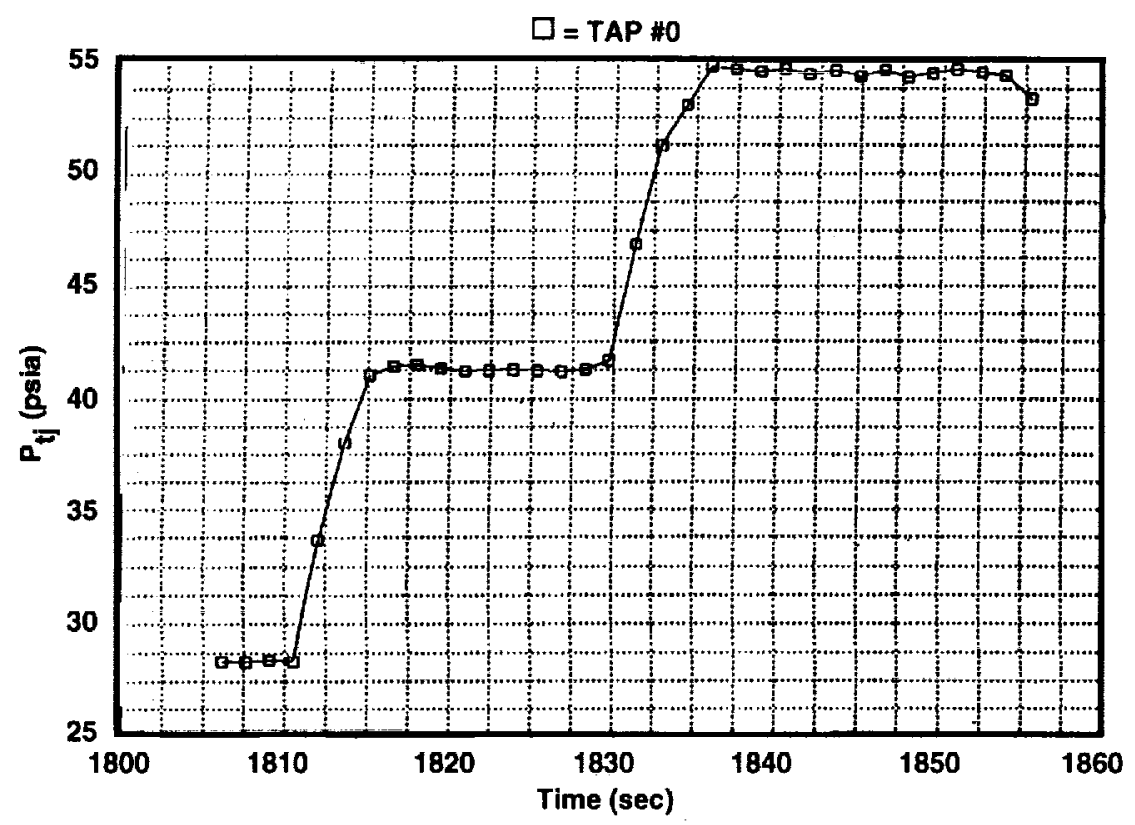

Figure 16. Samples of data obtained during a run in which three values of jet total pressure were set. (a) Jet total pressure.

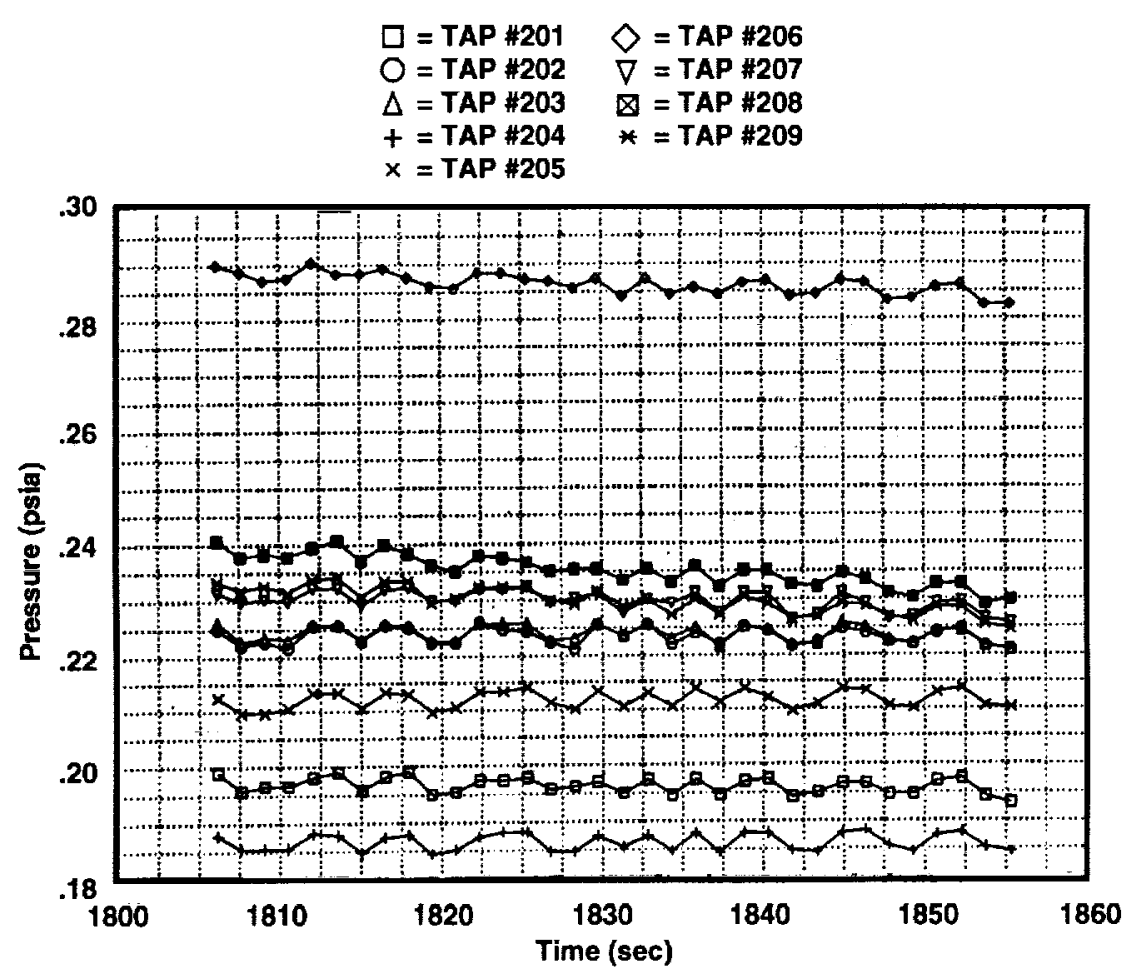

Figure 16. Continued. (b) Forebody static pressures. 


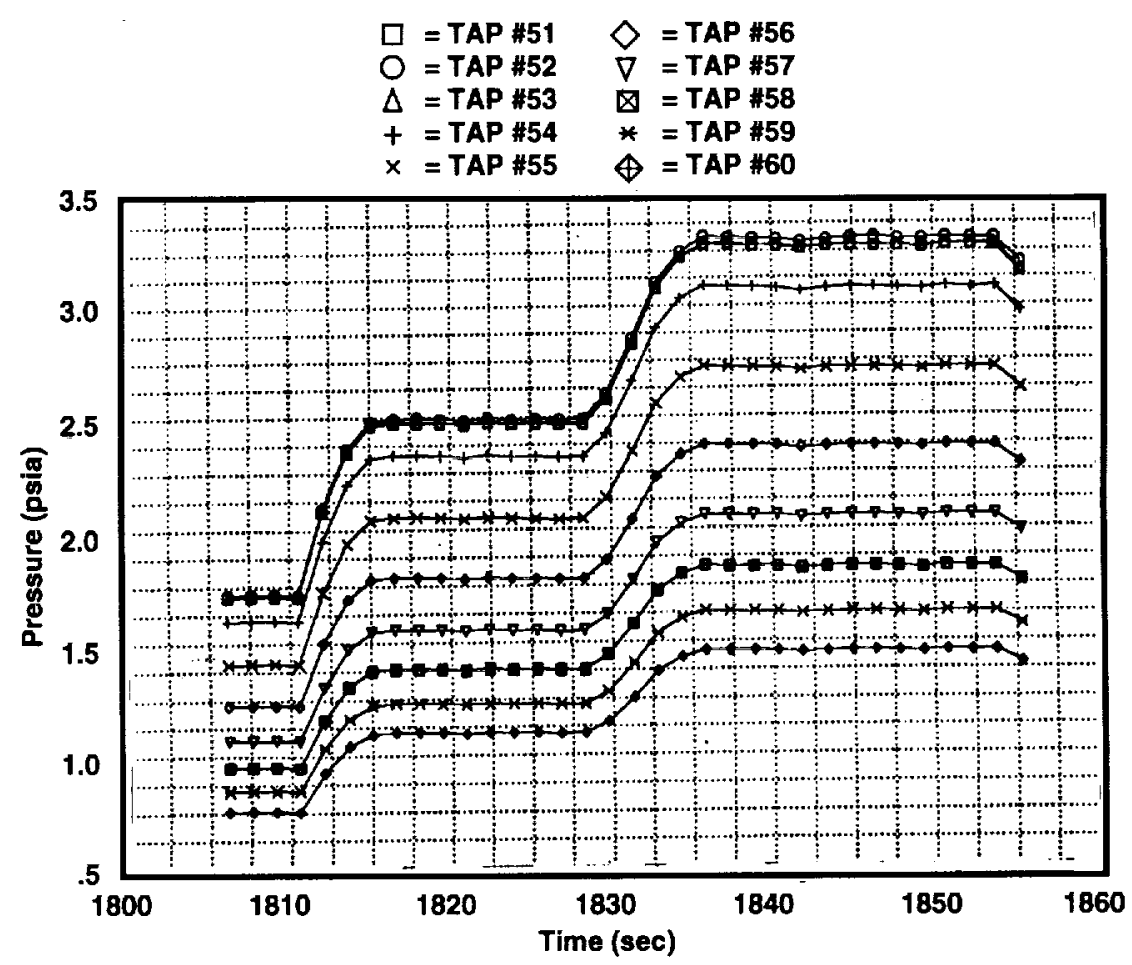

Figure 16. Continued. (c) Ramp centerline static pressures.

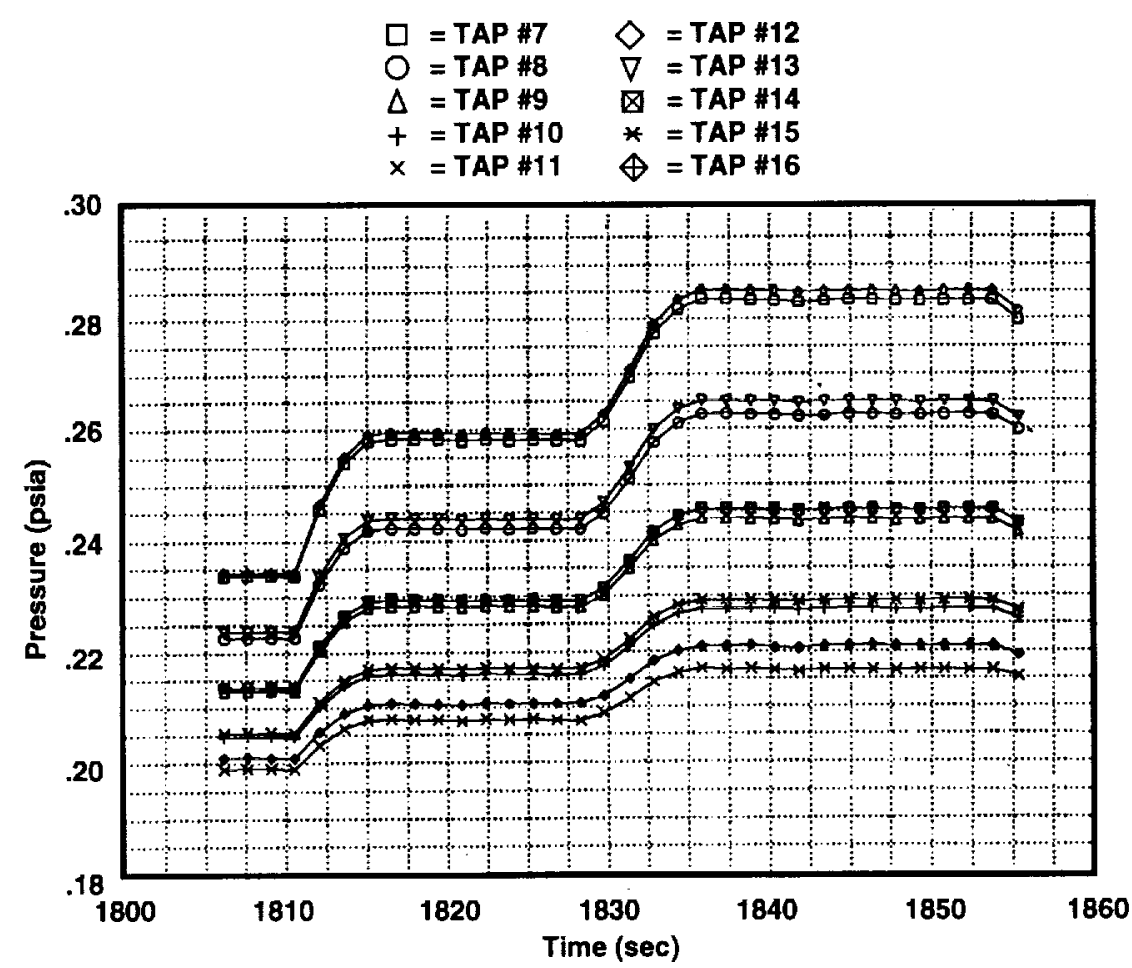

Figure 16. Continued. (d) Static pressures at combustor exit station and transition radius to ramp. 


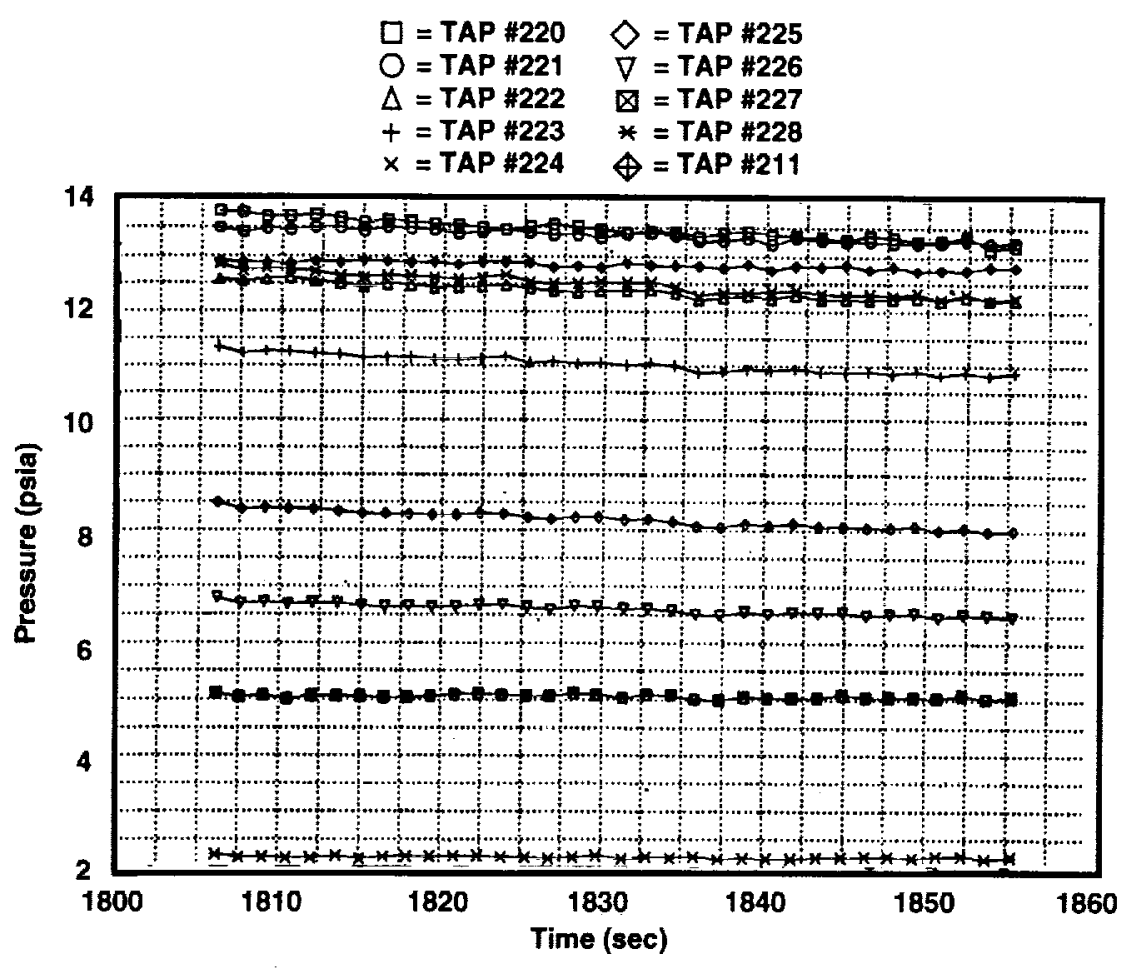

Figure 16. Continued. (e) Forebody boundary-layer rake.

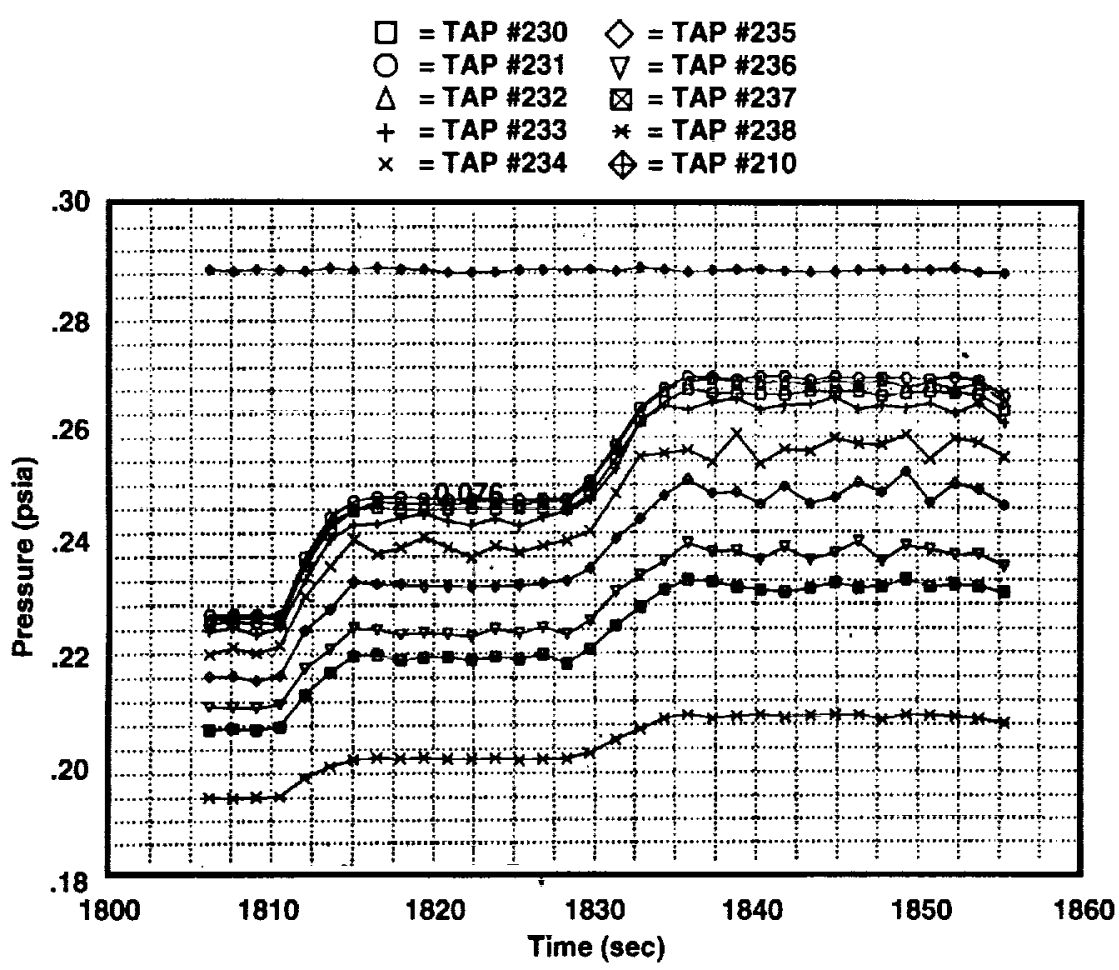

Figure 16. Continued. (f) Forward ramp boundary-layer rake. 


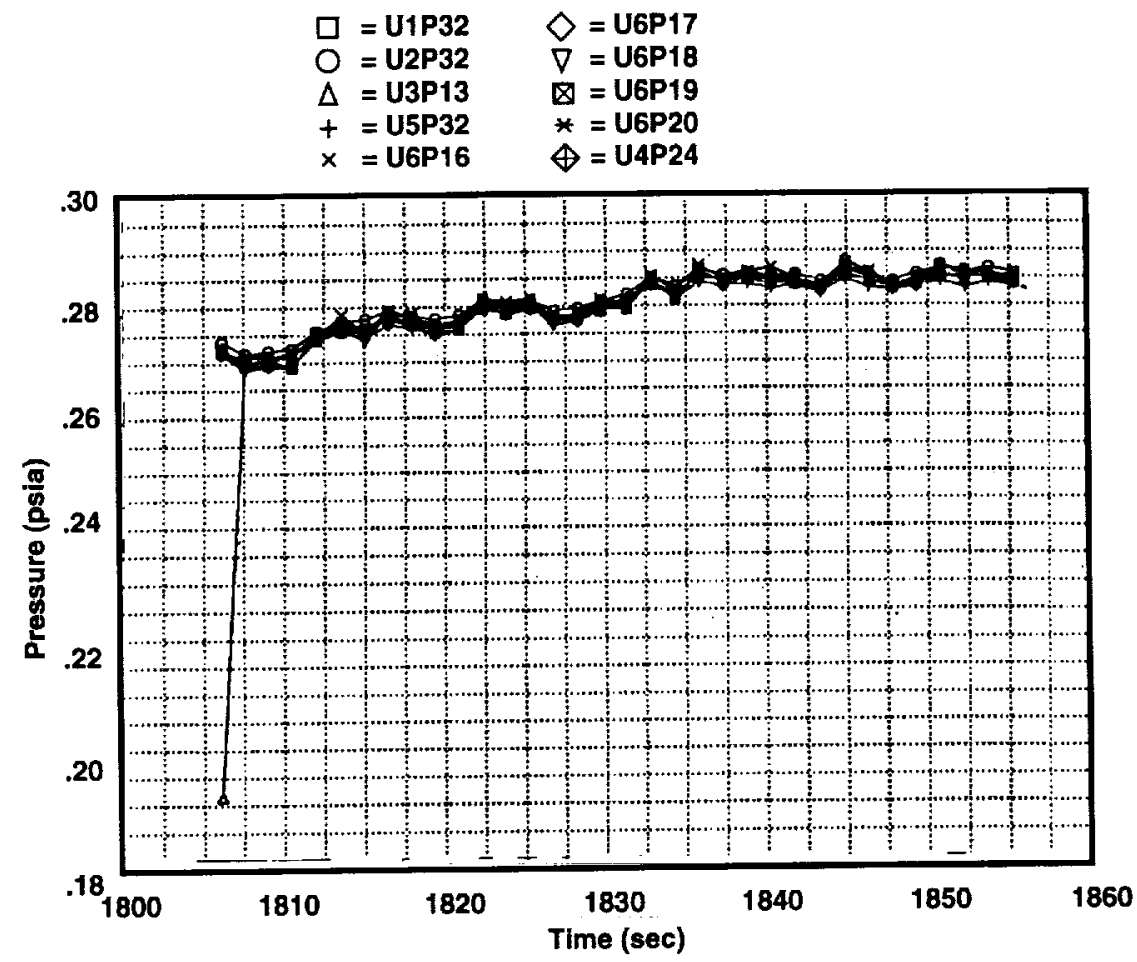

Figure 16. Concluded. (g) Transducers open to model internal pressure.
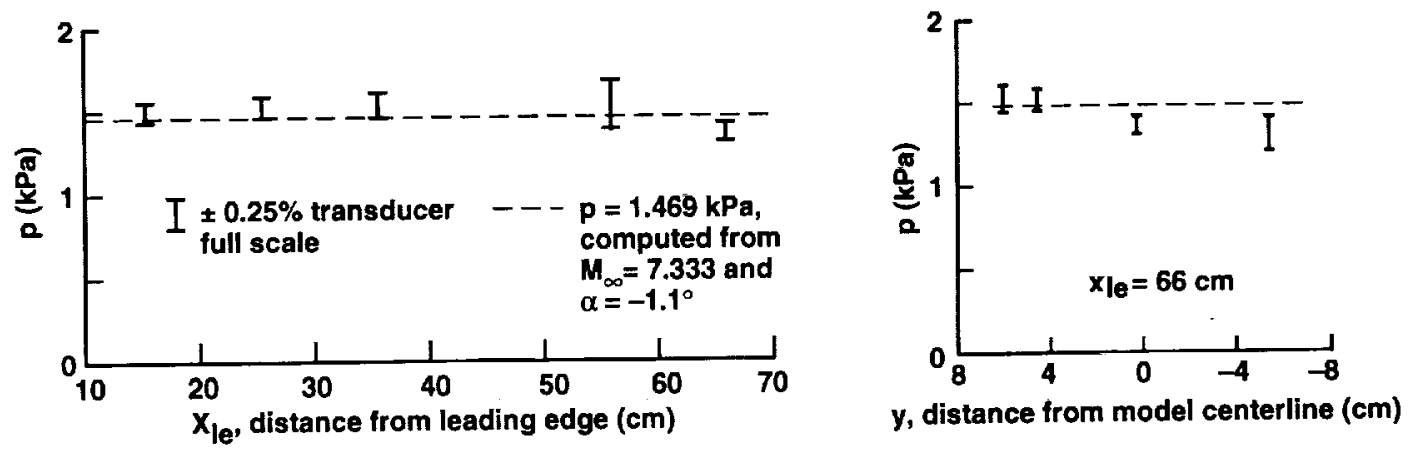

Figure 17. Forebody static pressure distributions. 


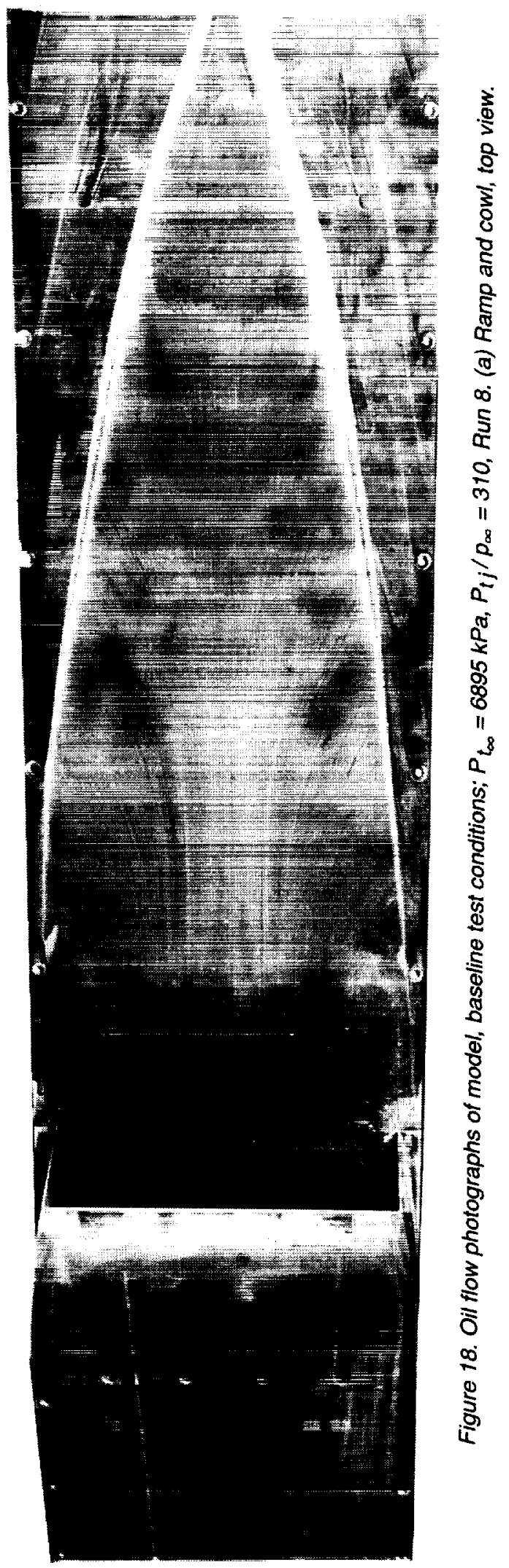




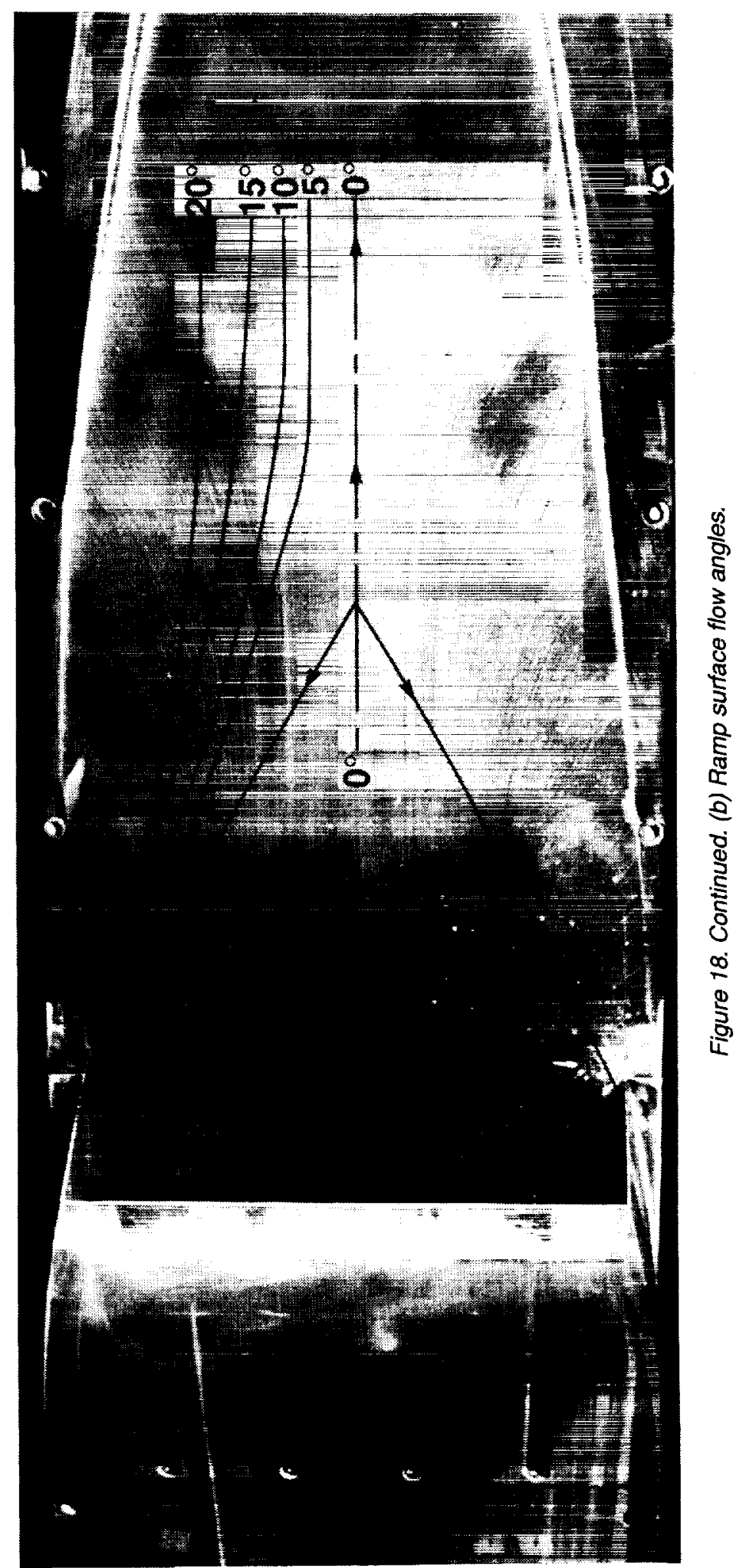



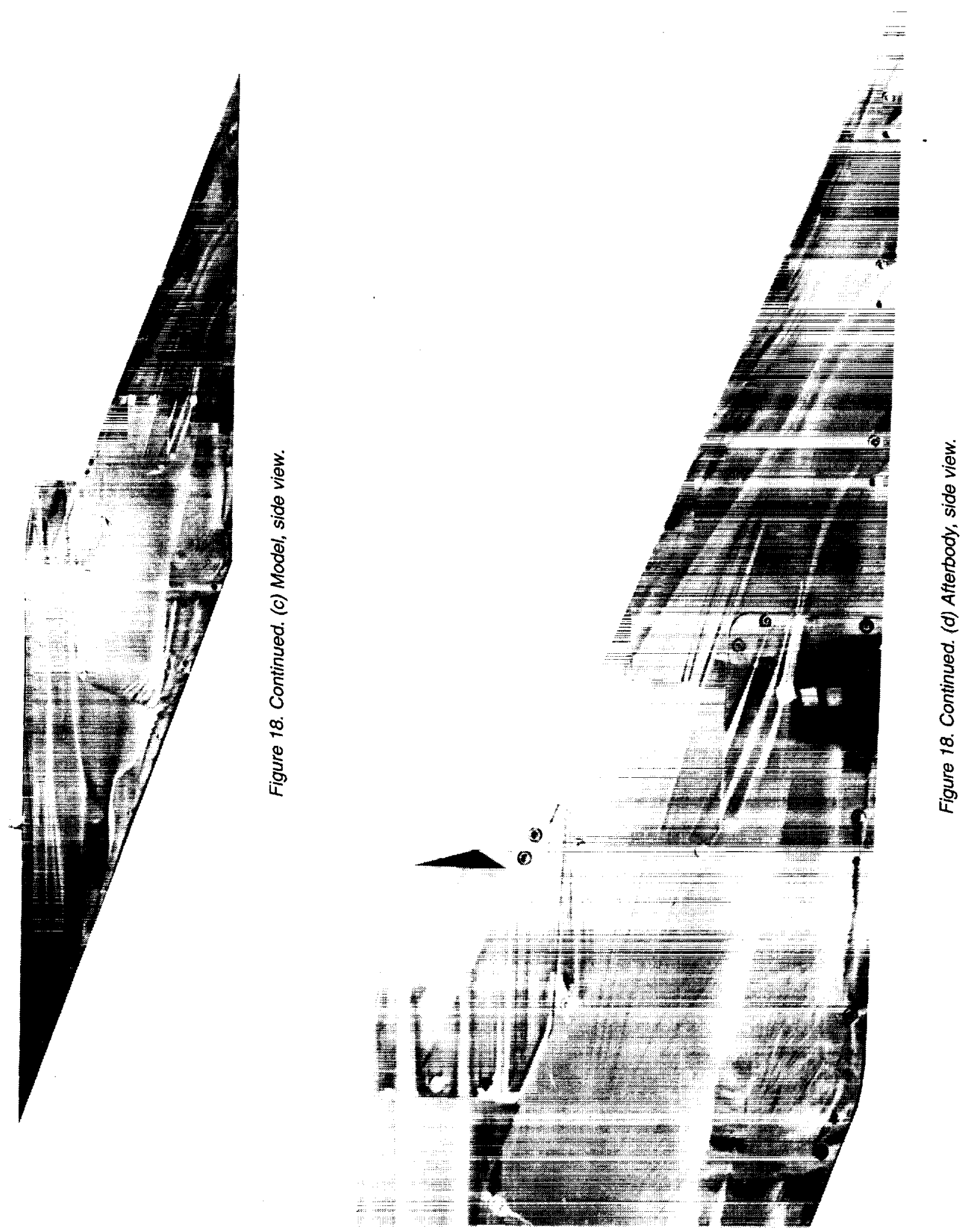


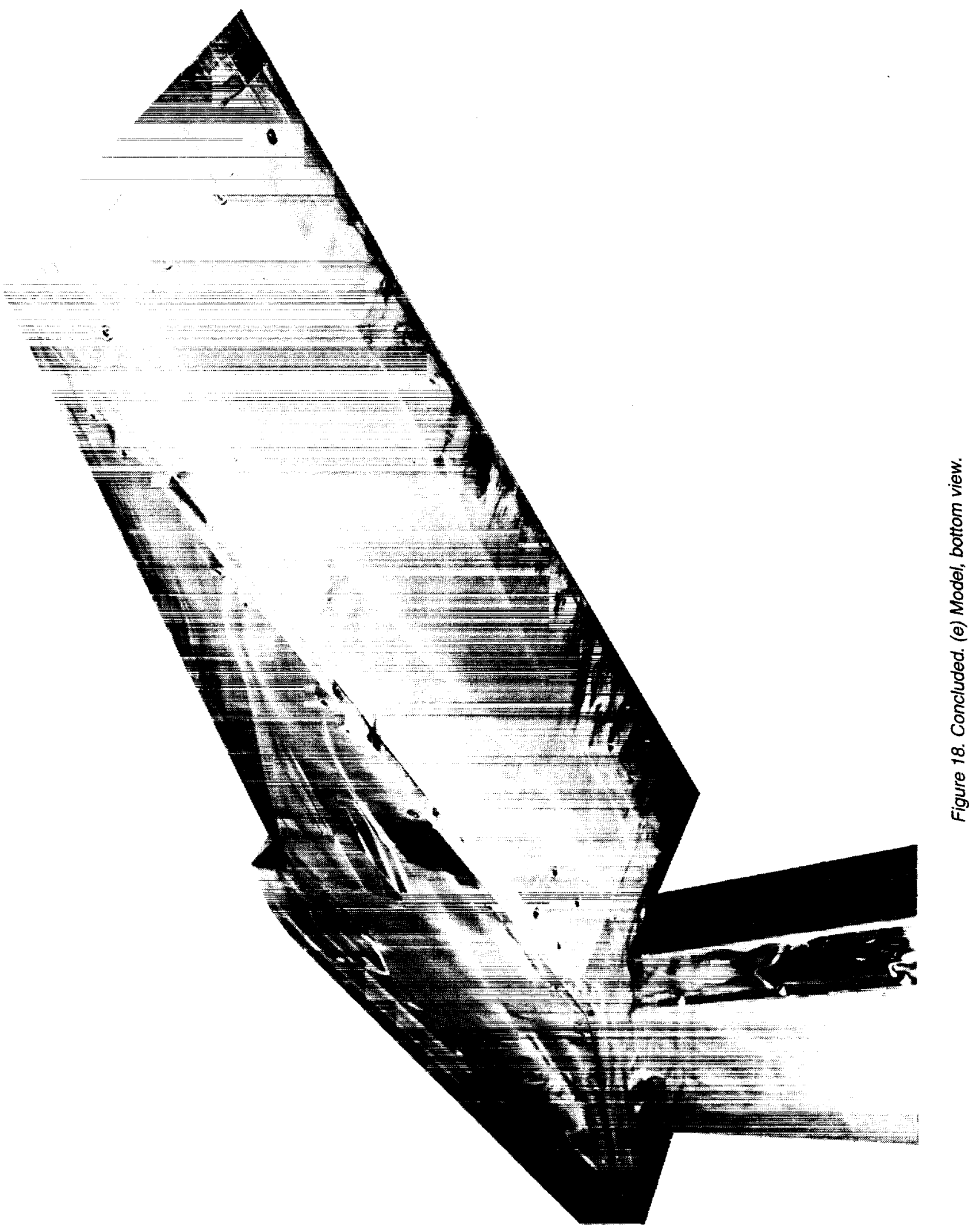




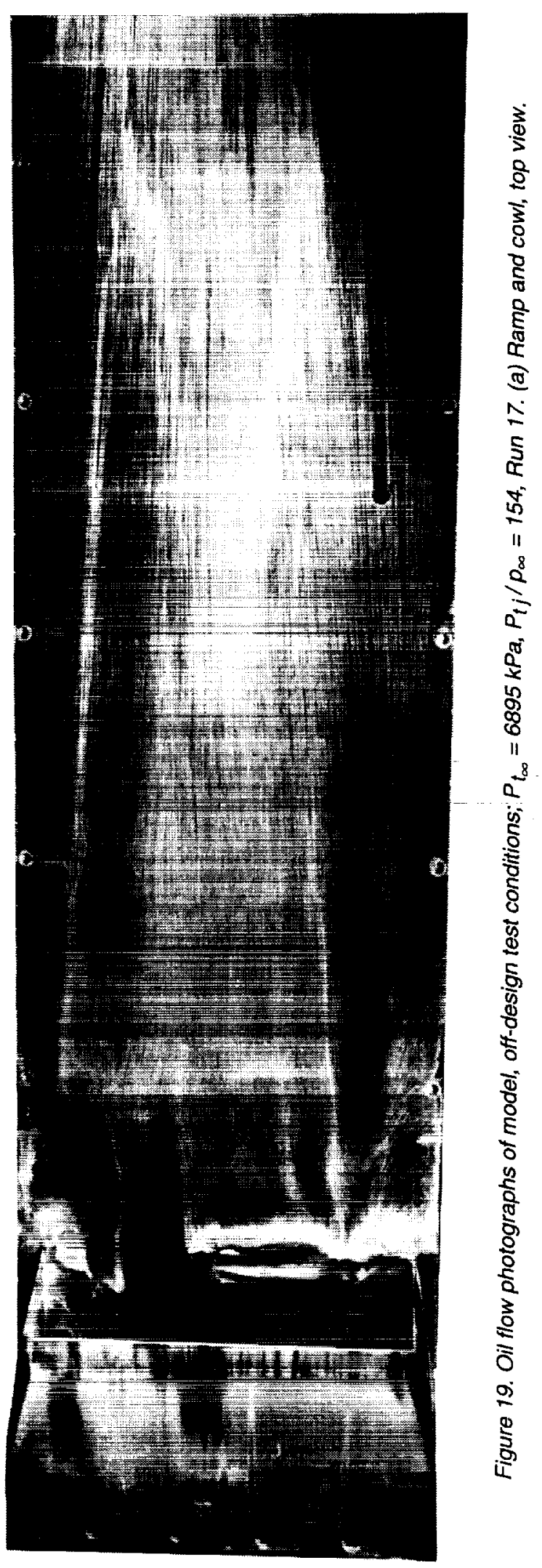




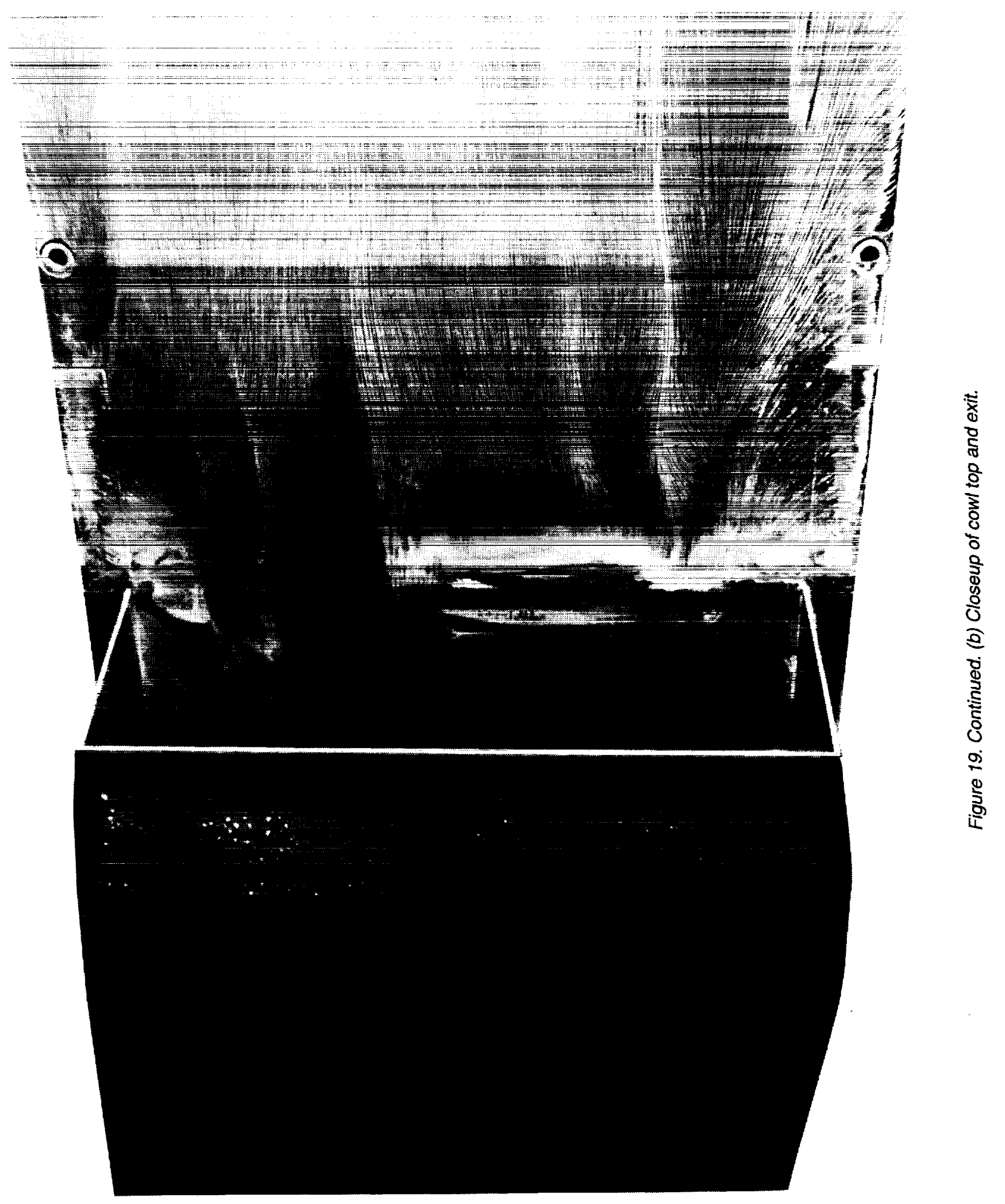




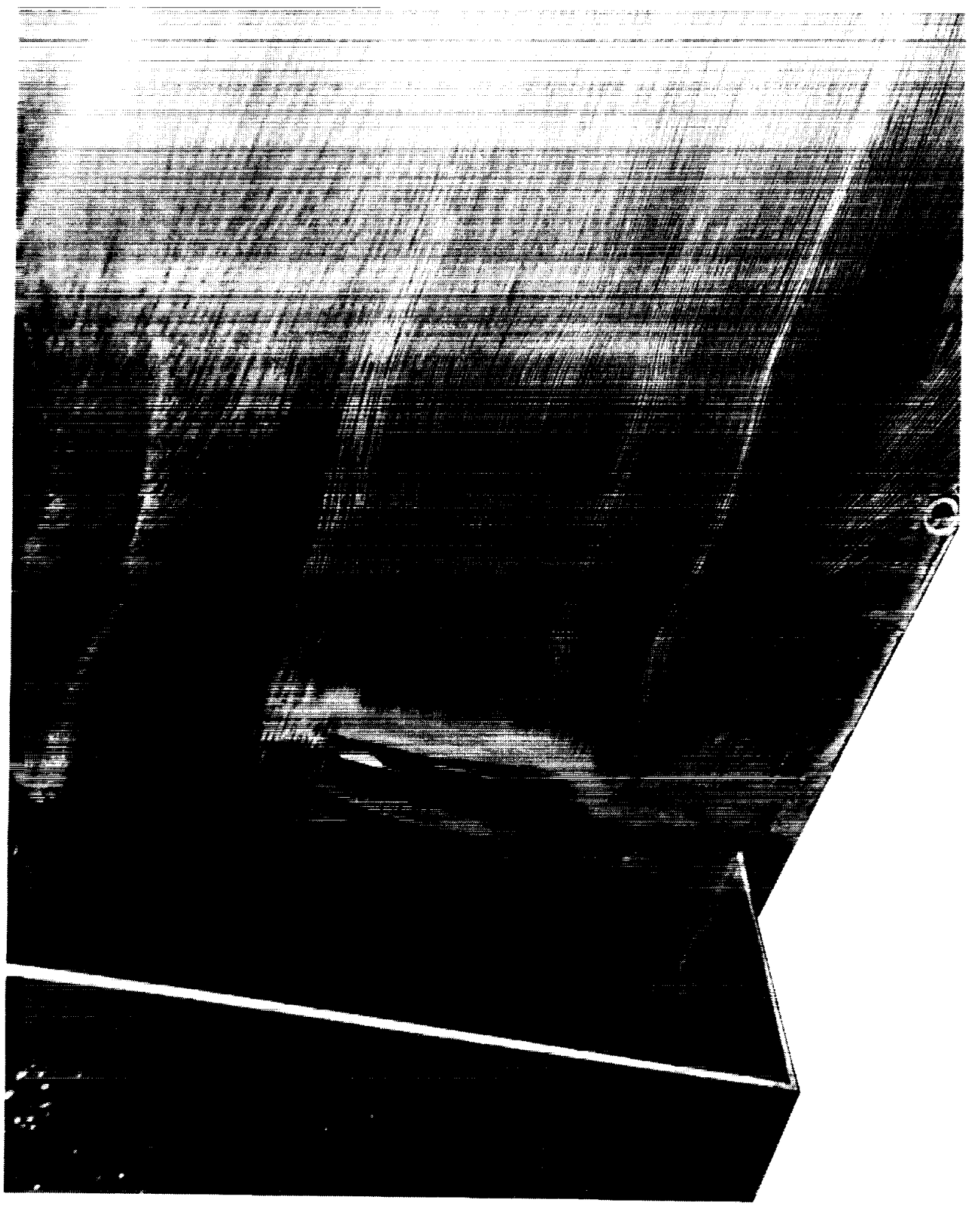




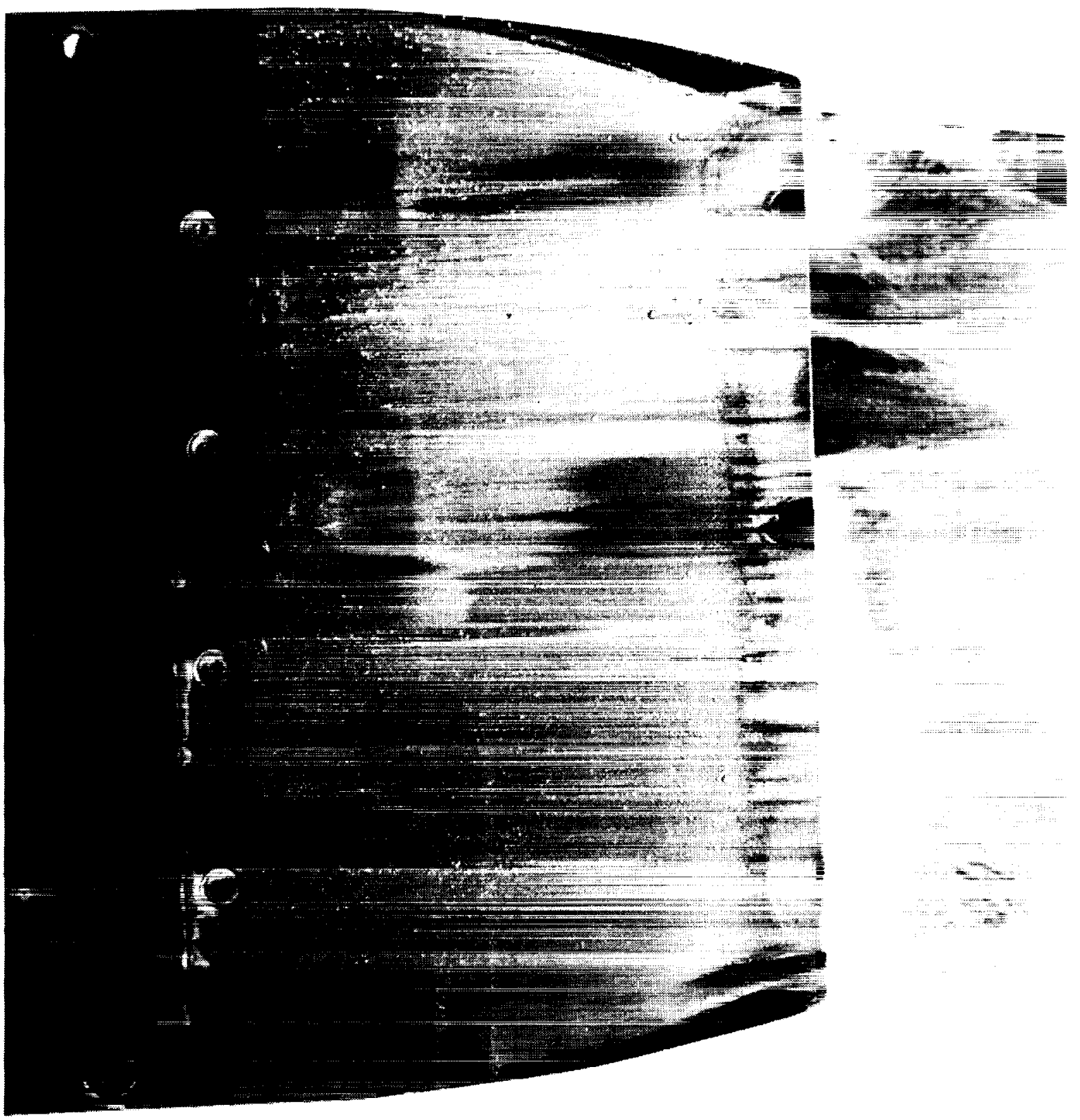

Figure 19. Continued. (d) Closeup of cowl top. 


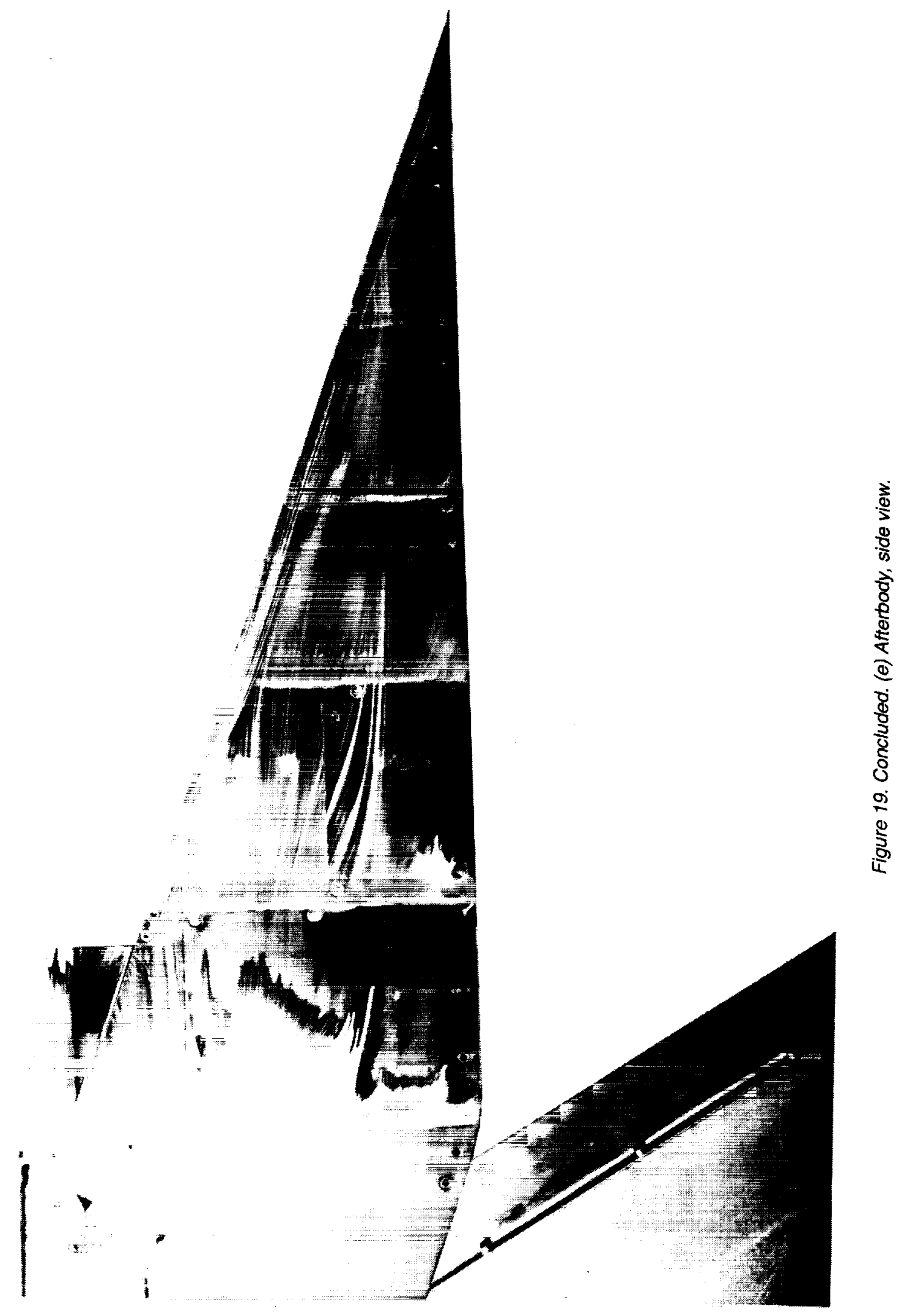




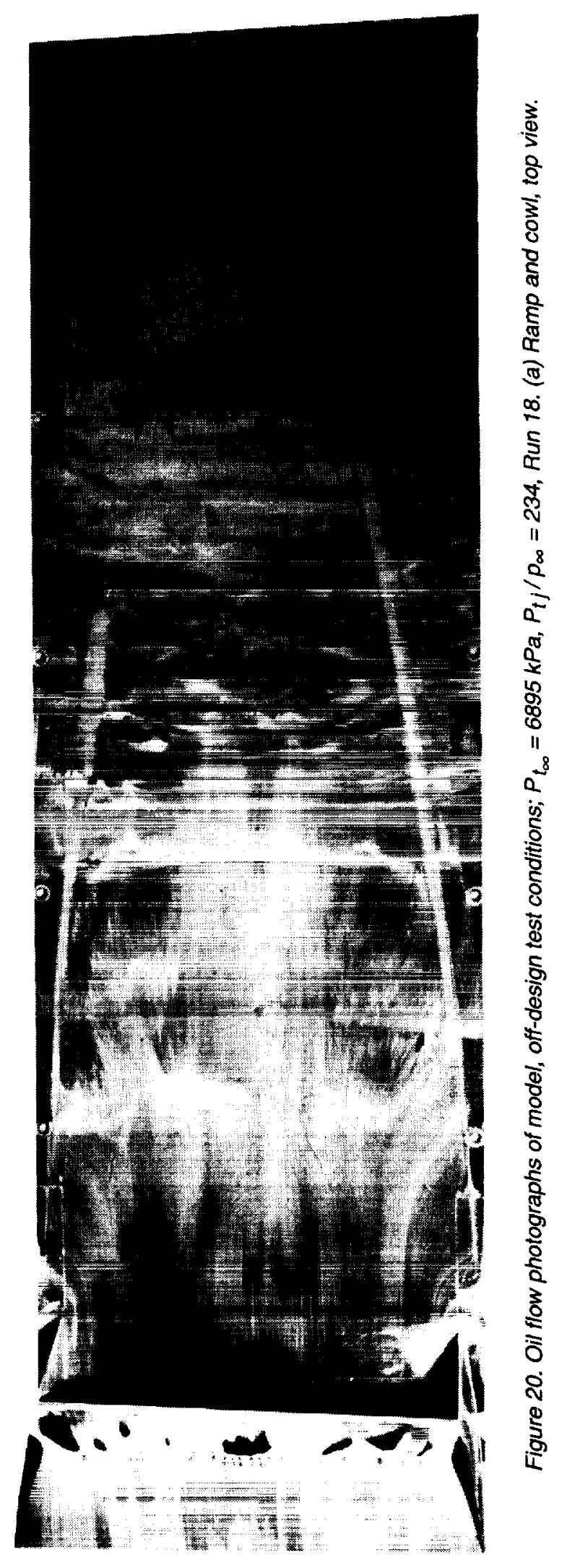




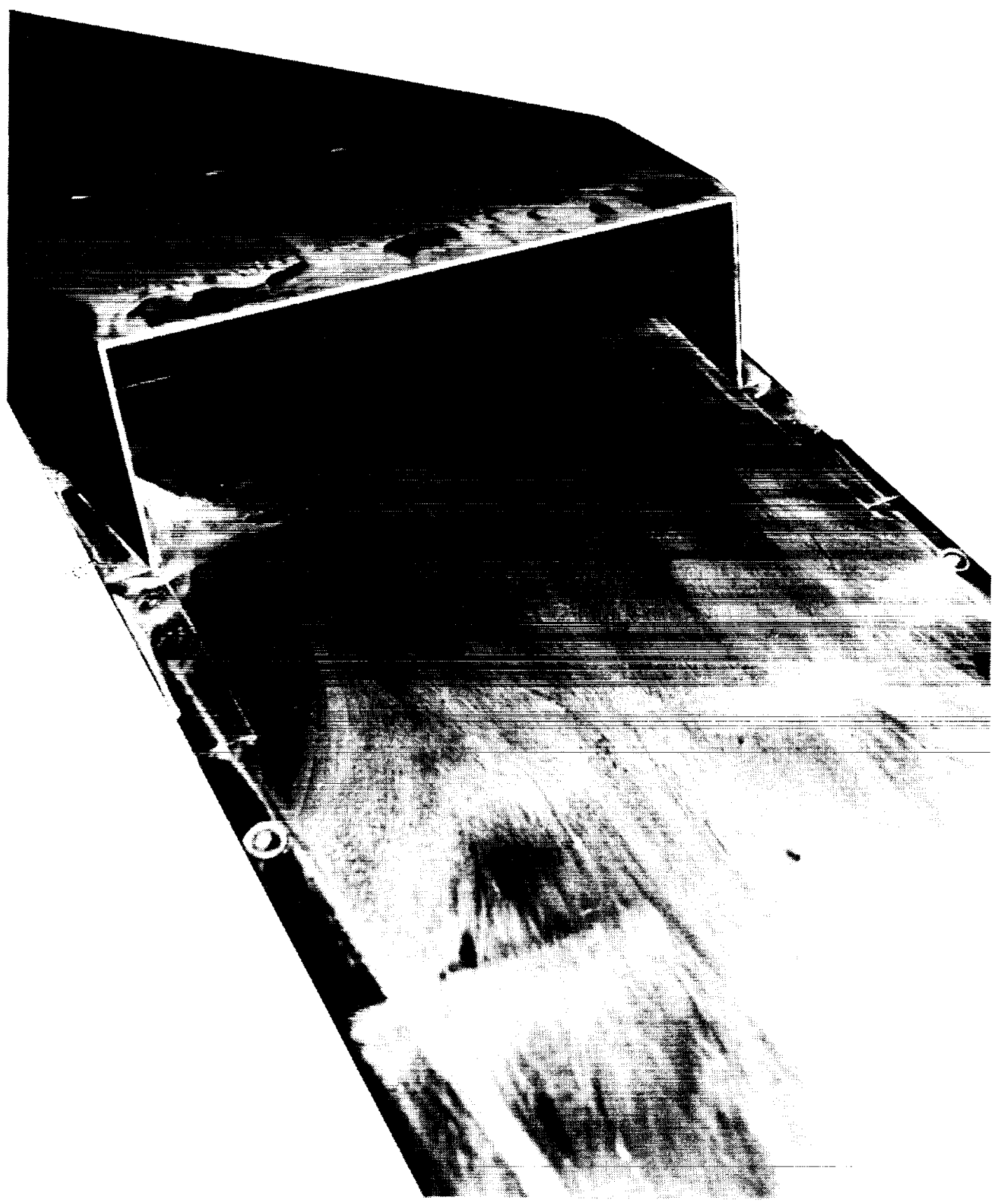

Figure 20. Continued. (b) Closeup of cowl top and exit. 


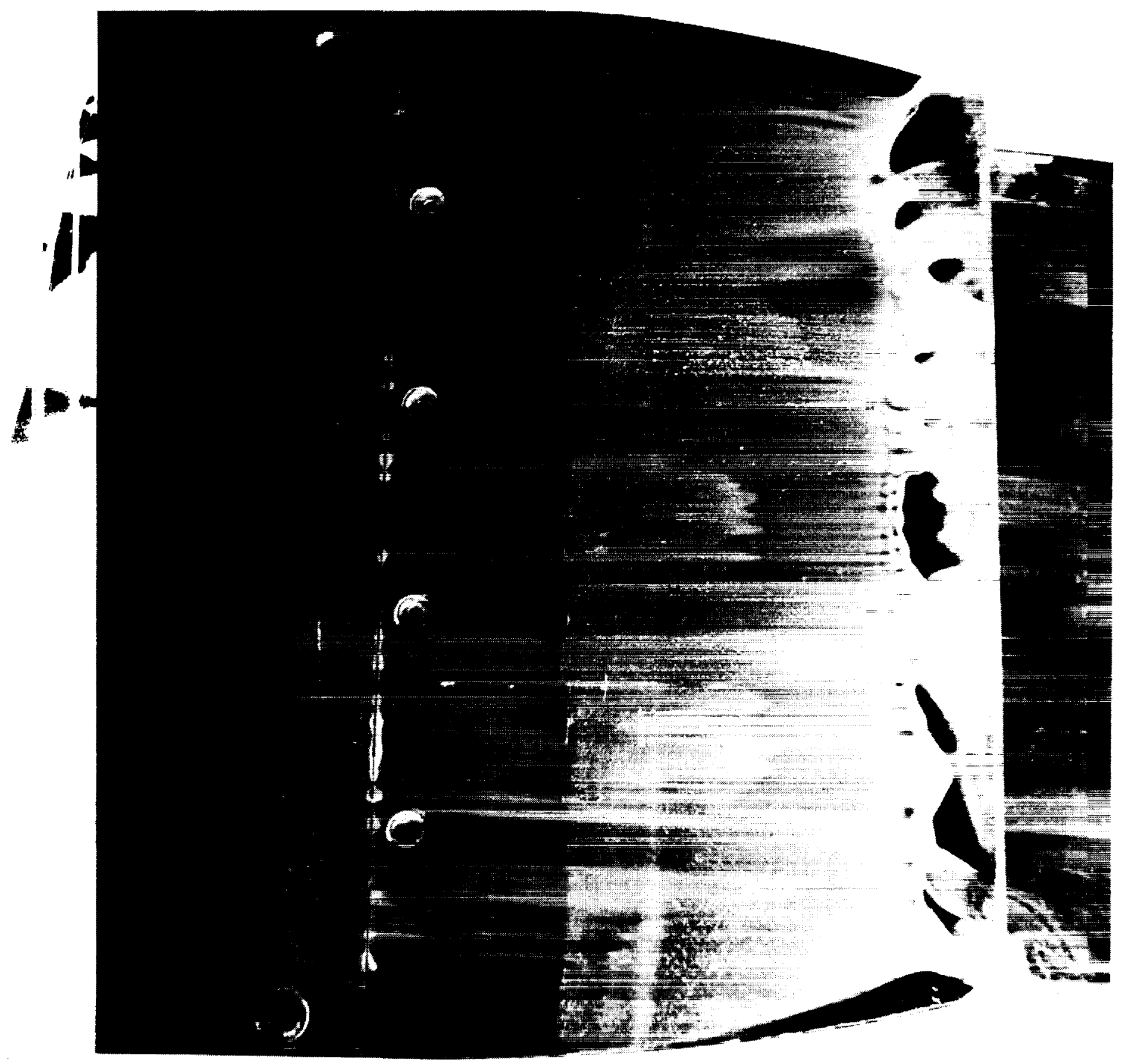

Figure 20. Continued. (c) Closeup of cowl top. 


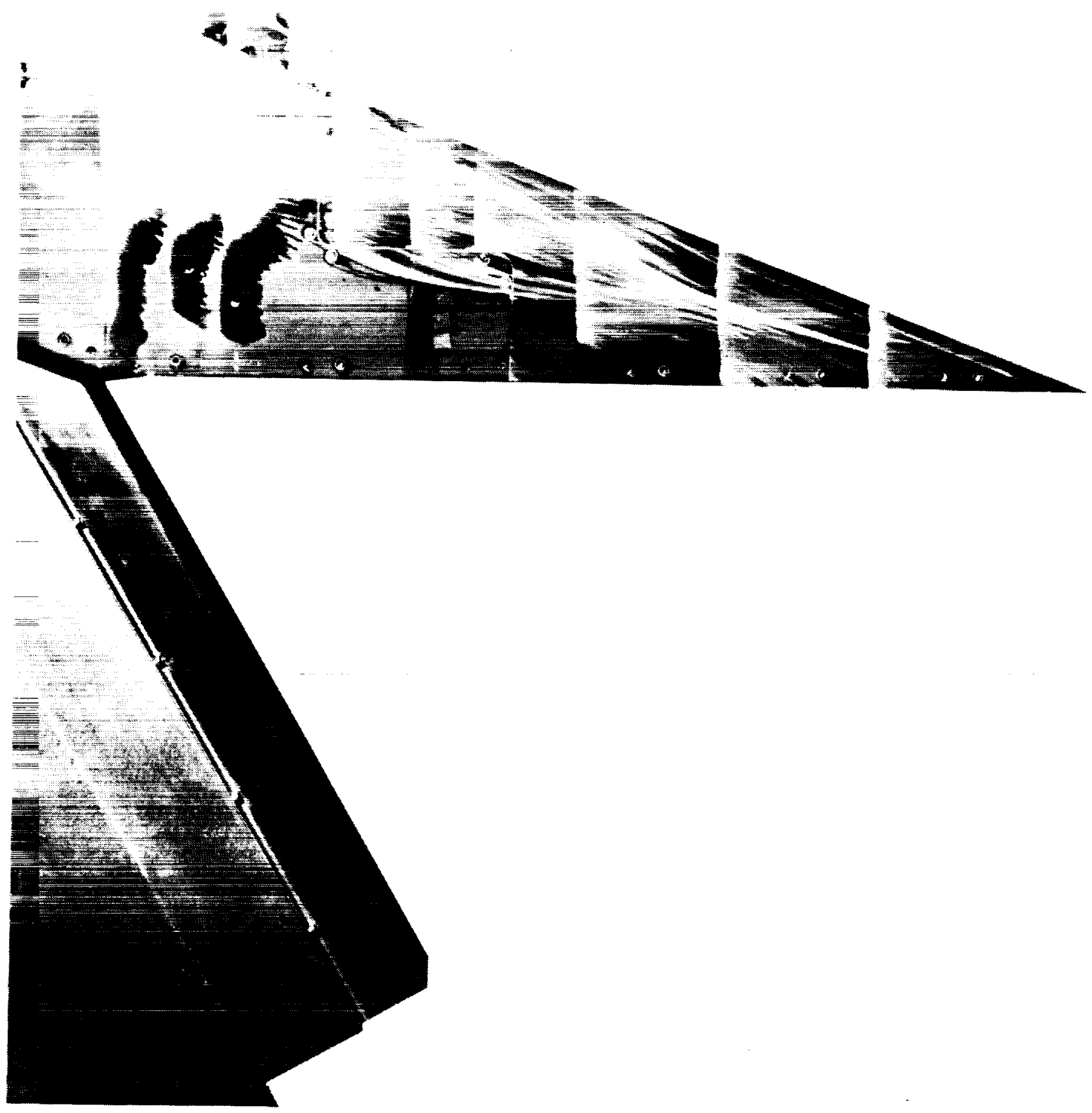

Figure 20. Concluded. (d) Afterbody, side view. 


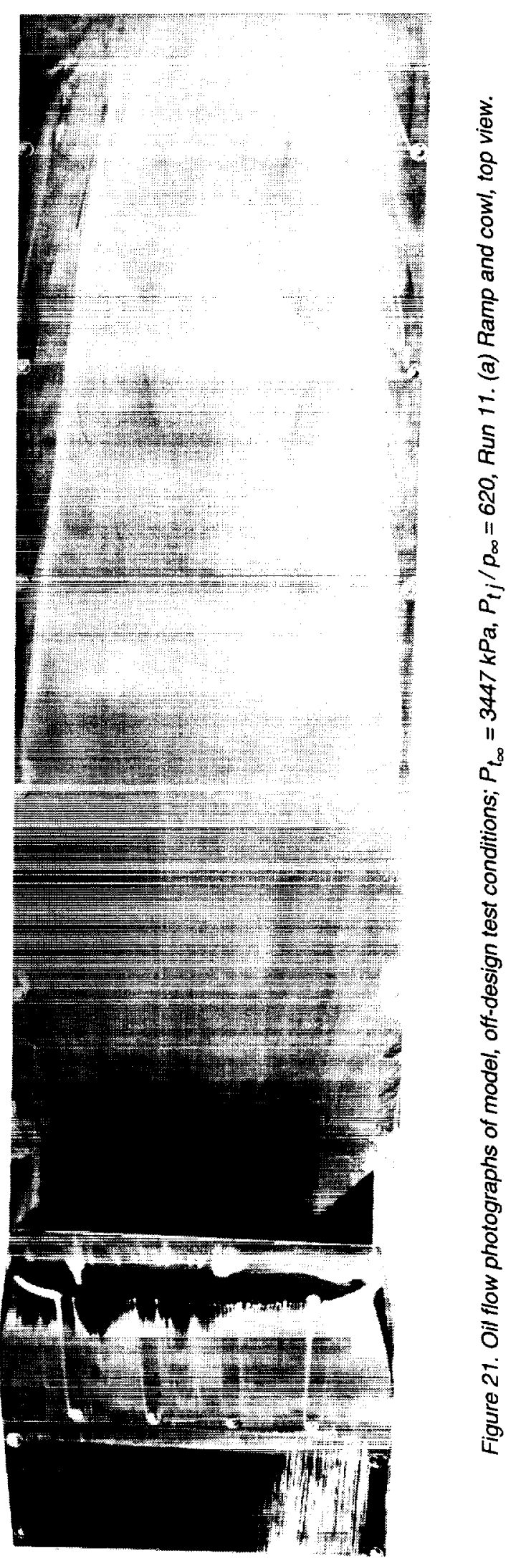




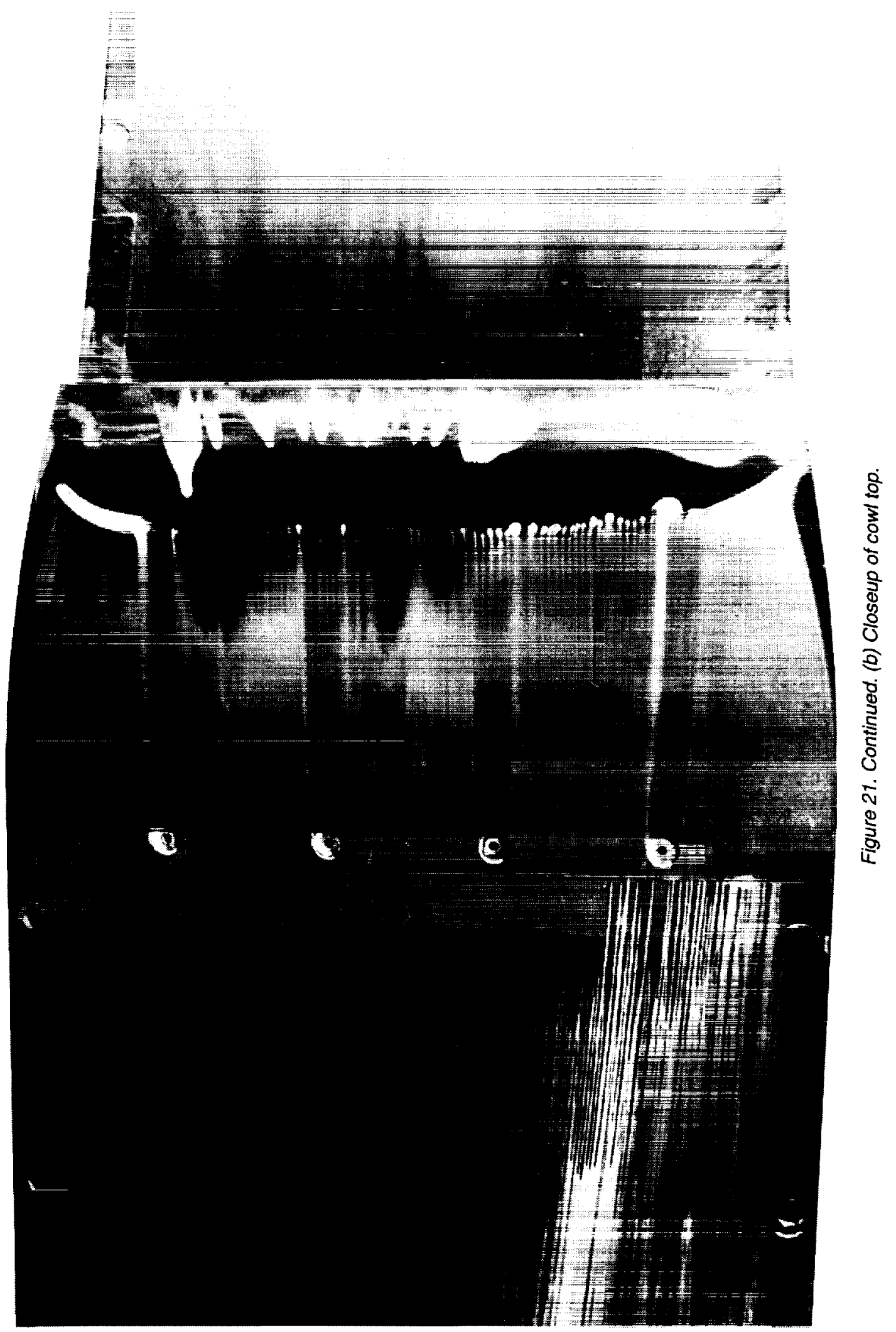




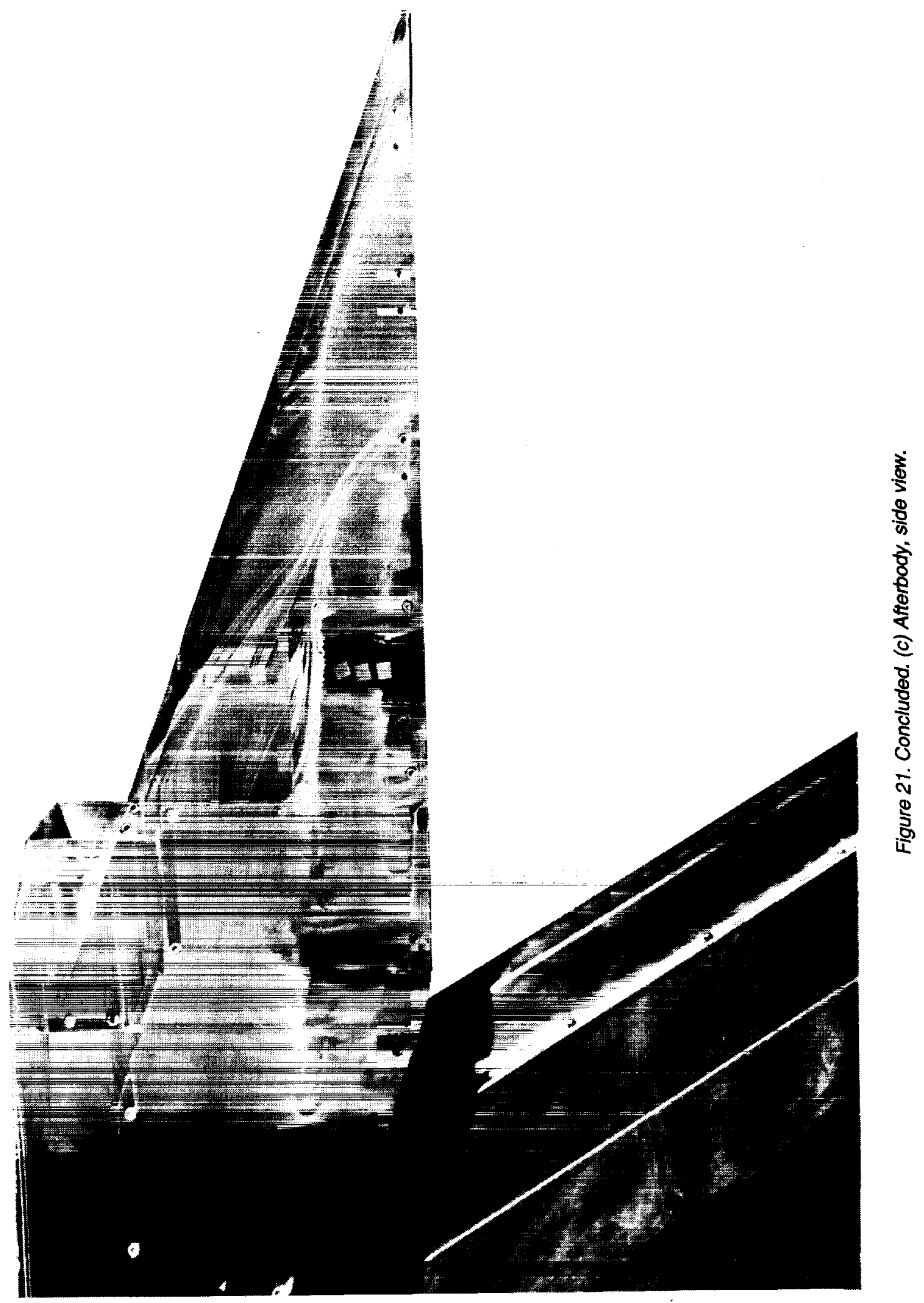




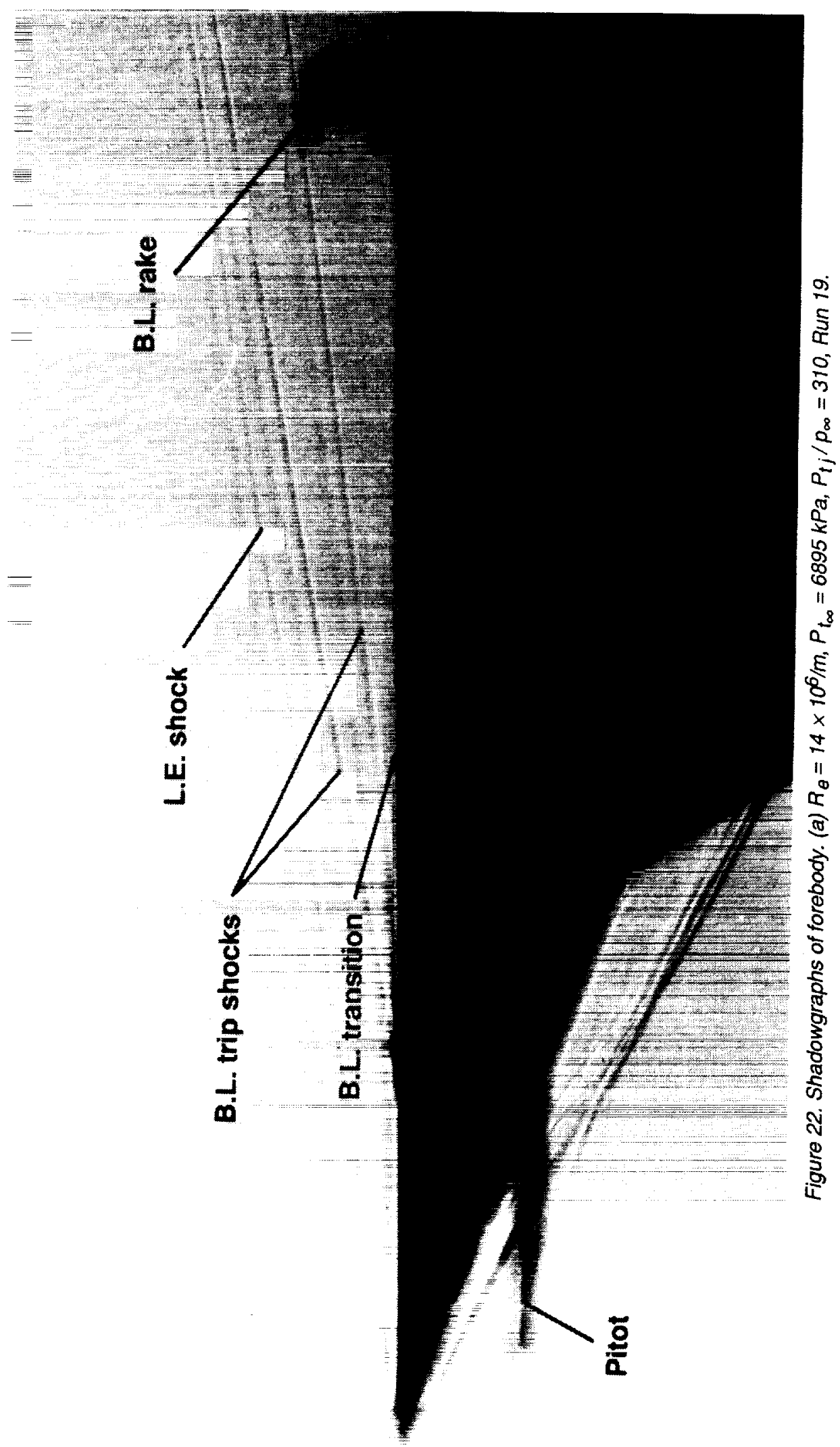




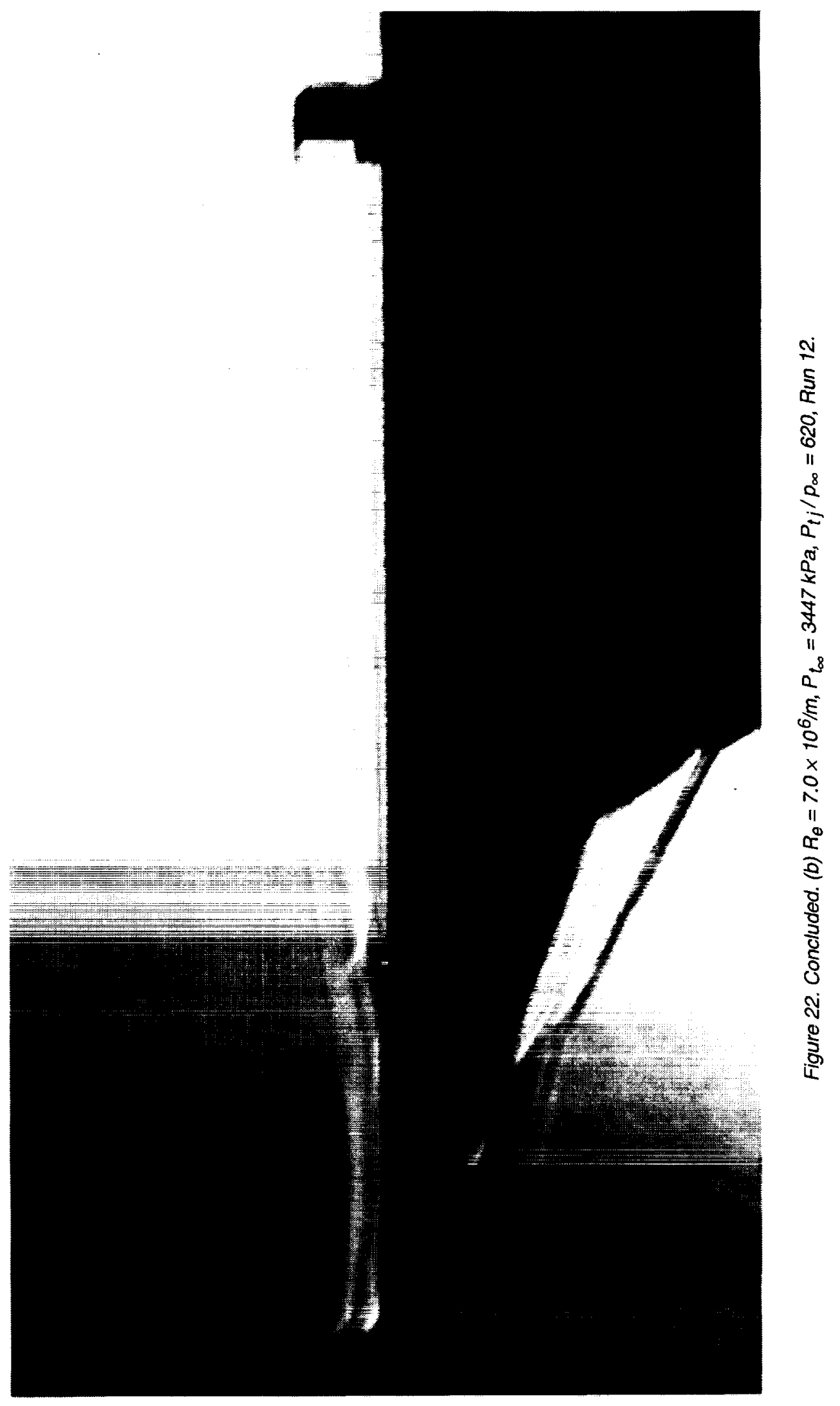




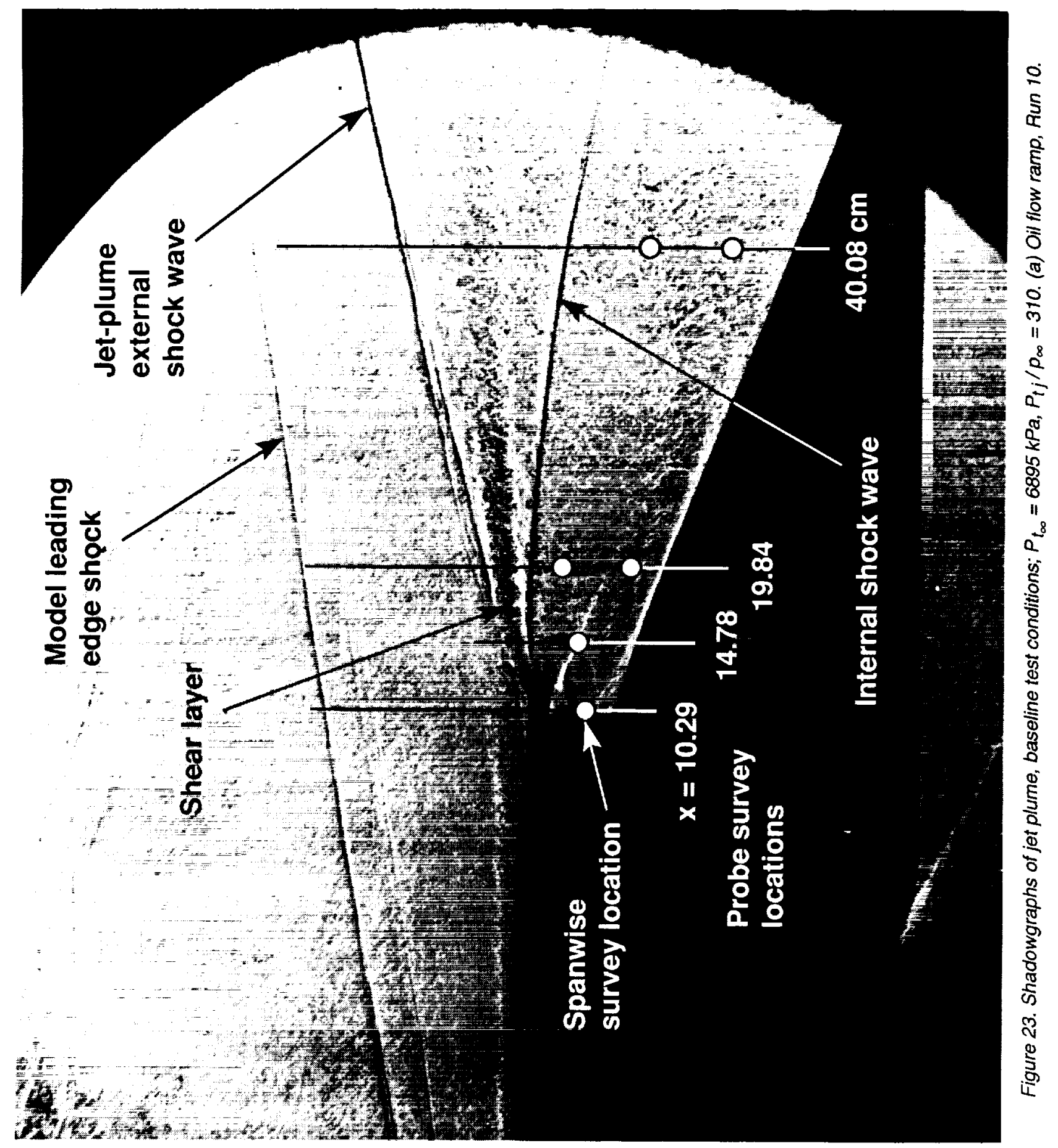




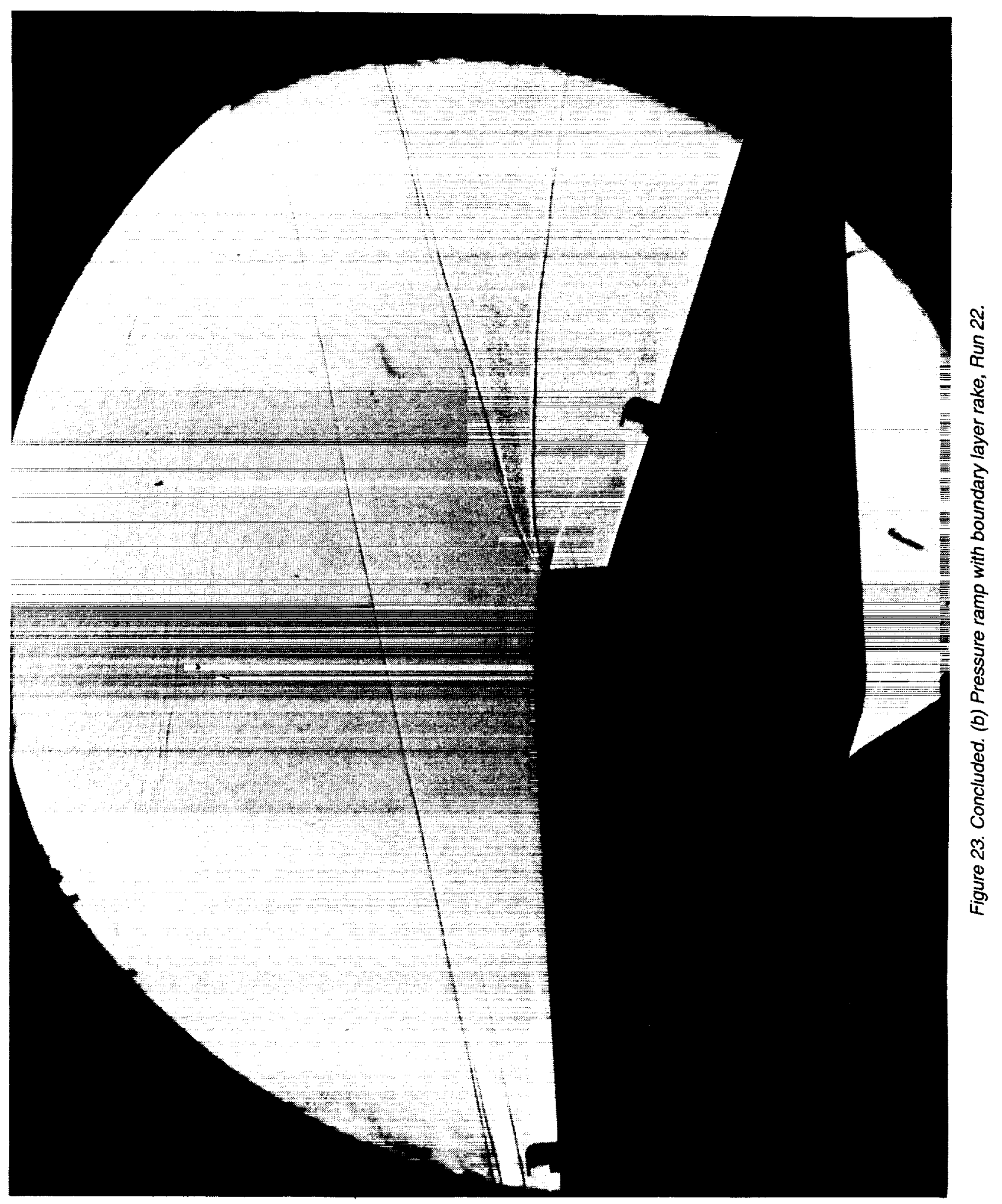




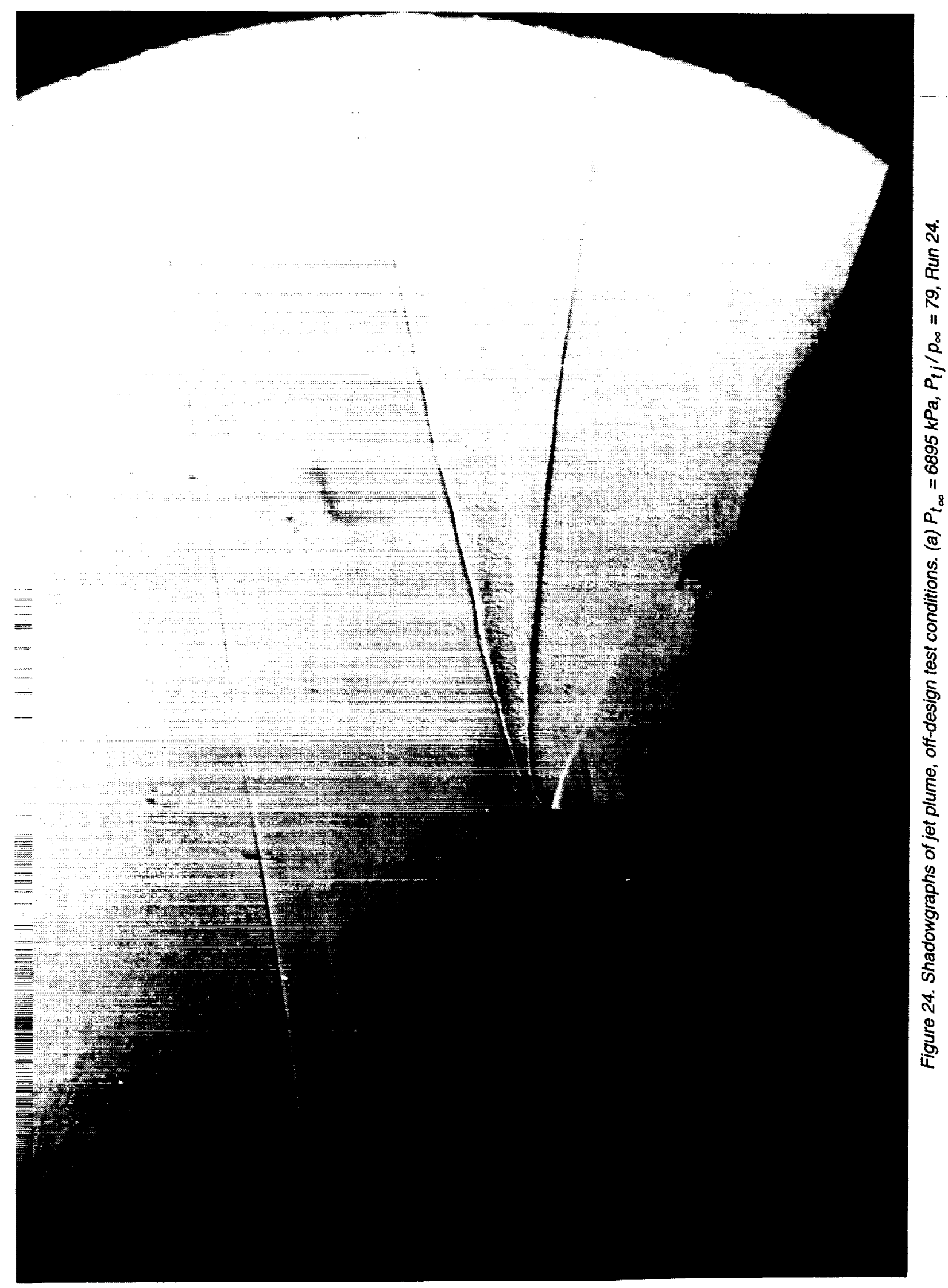




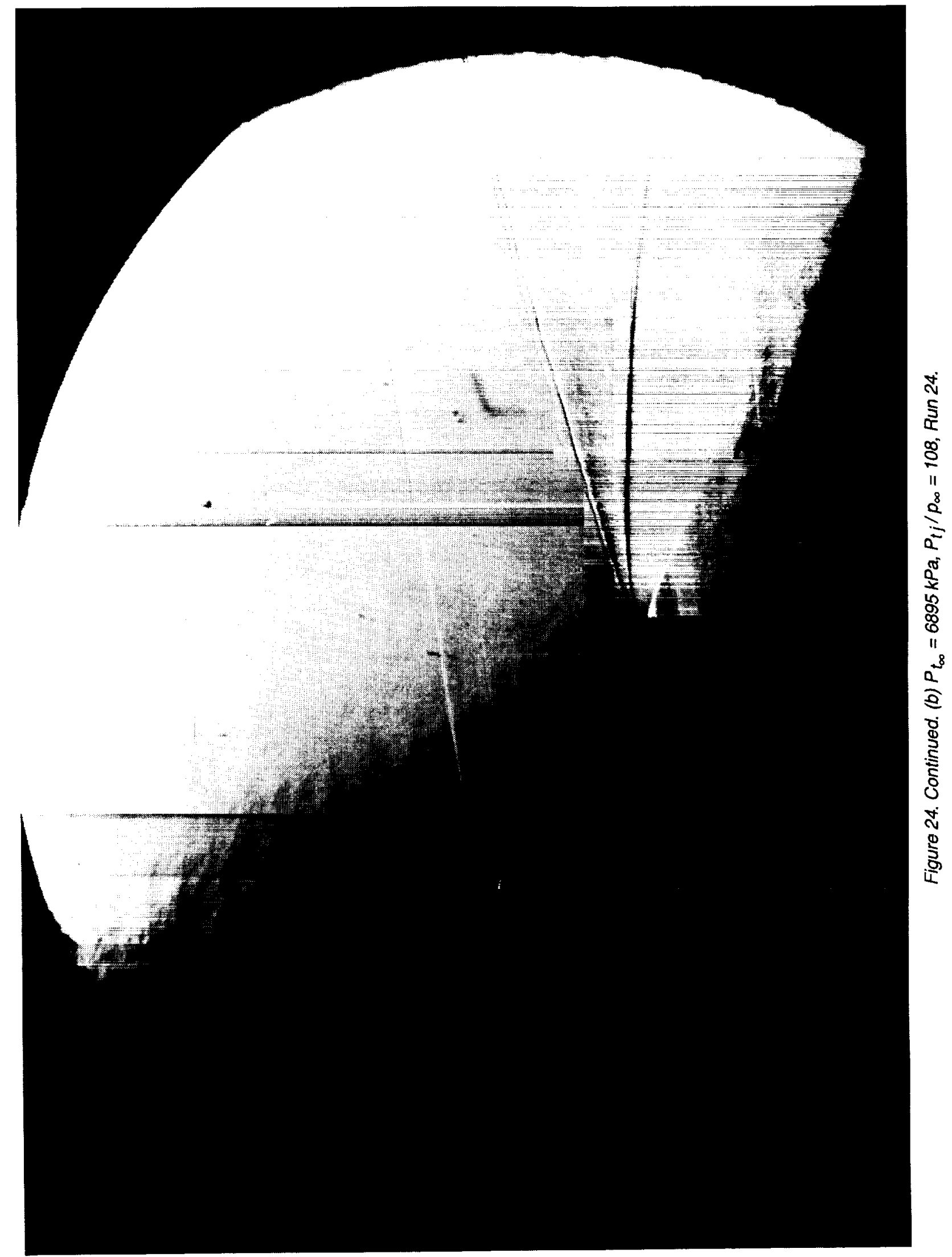




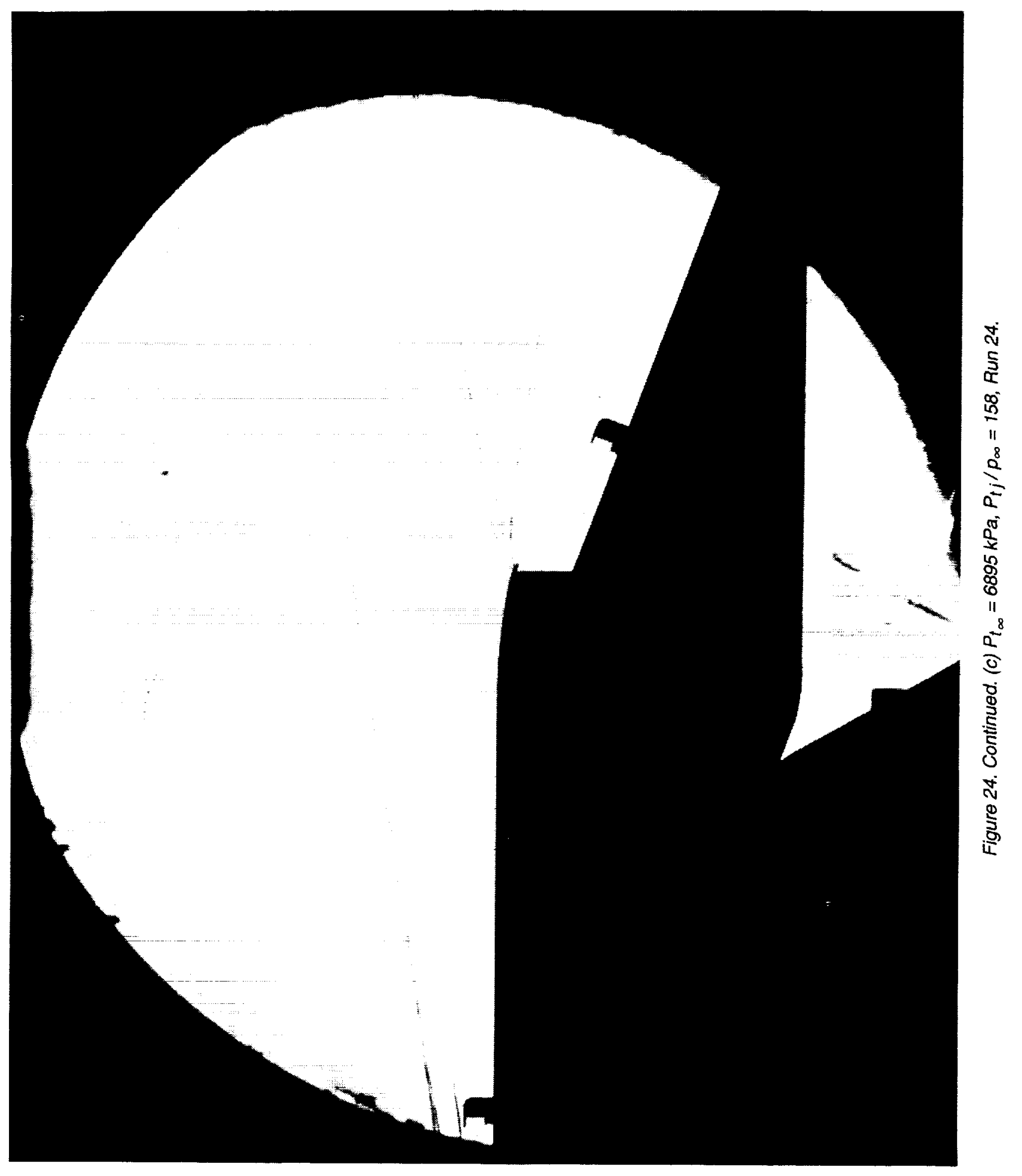




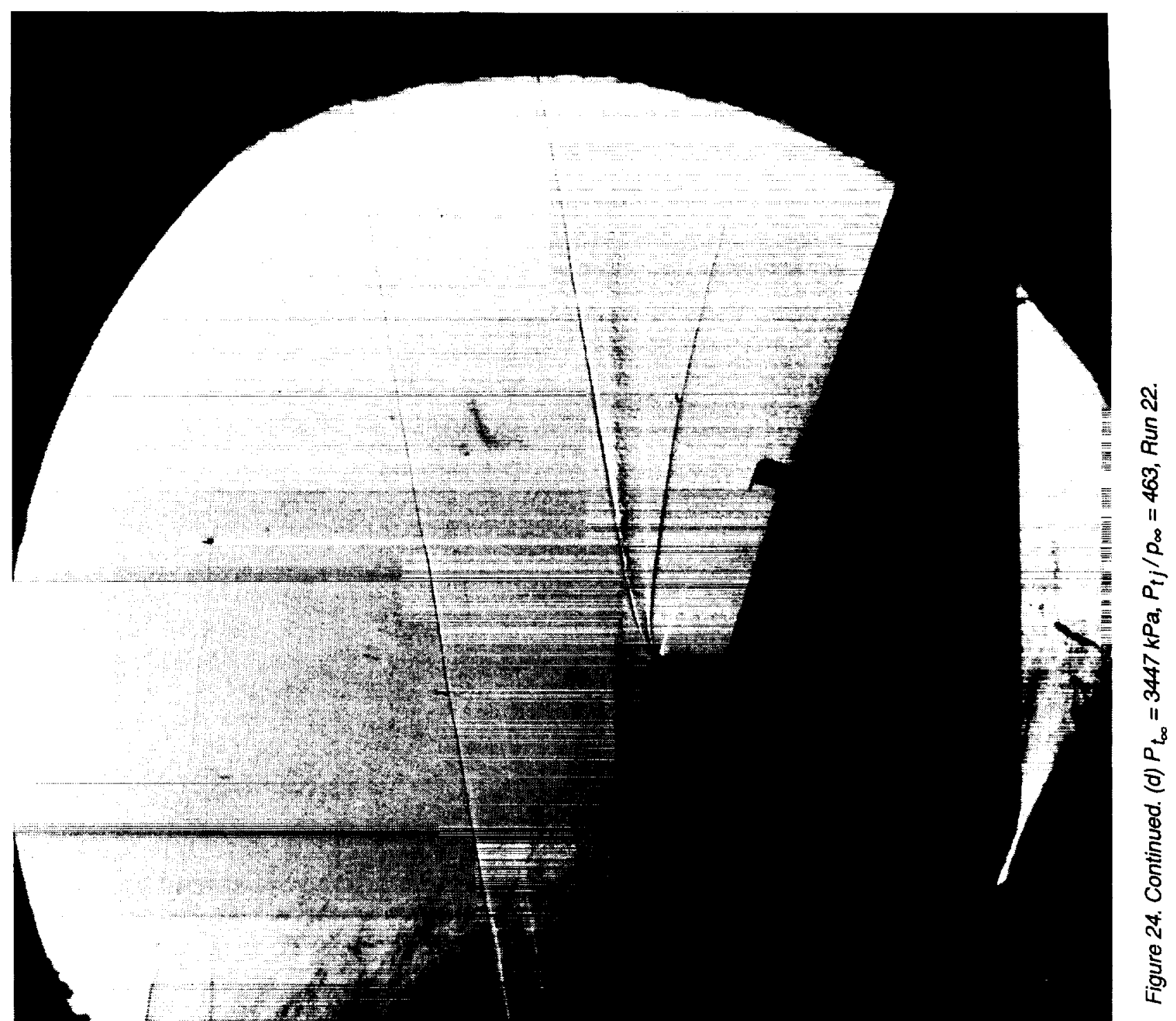




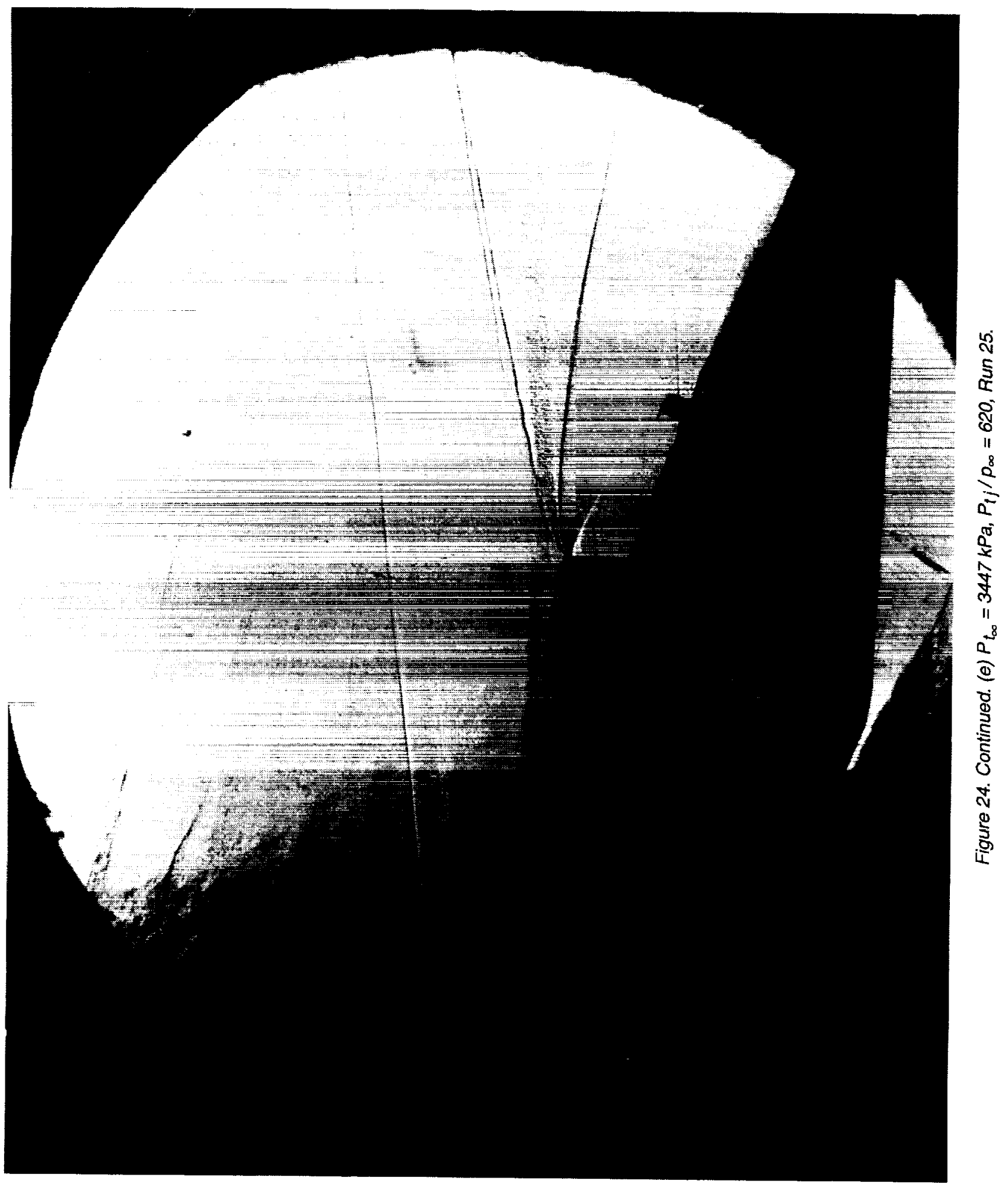




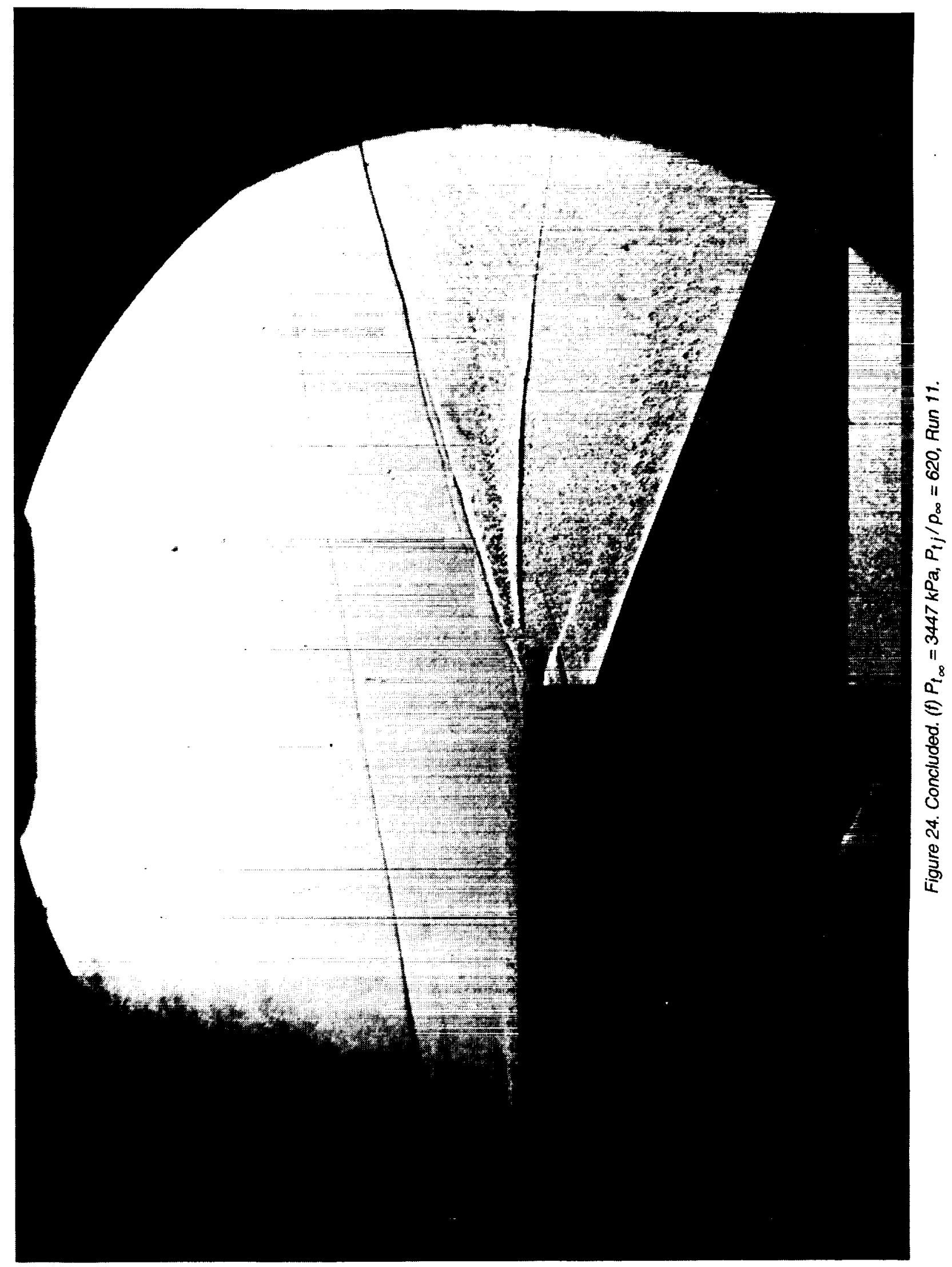



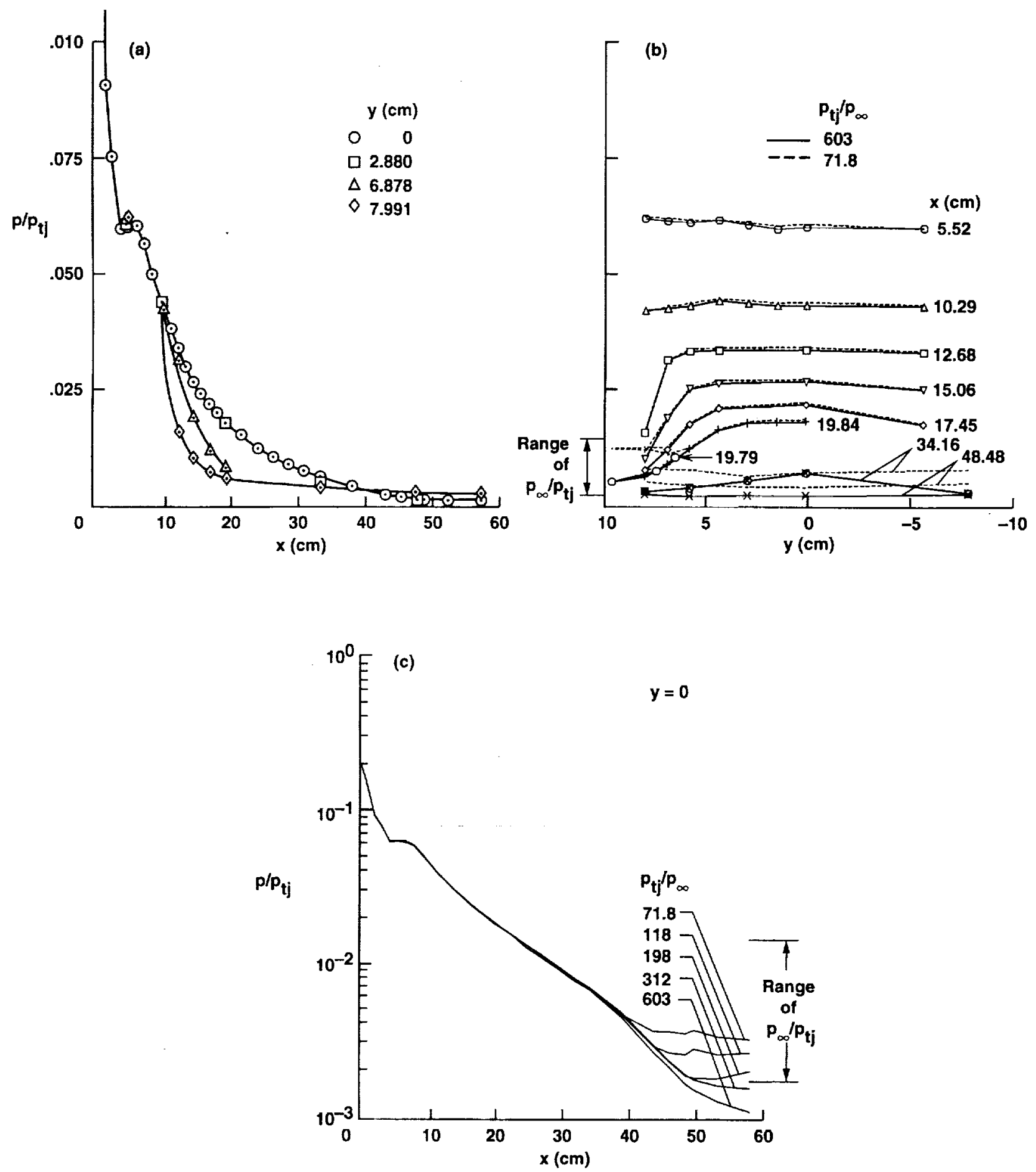

Figure 25. Ramp static pressure distributions. (a) Streamwise distributions at various values of $y$, baseline conditions; (b) transverse distributions at the maximum and minimum pressure ratios; (c) streamwise centerline distributions for various pressure ratios. 


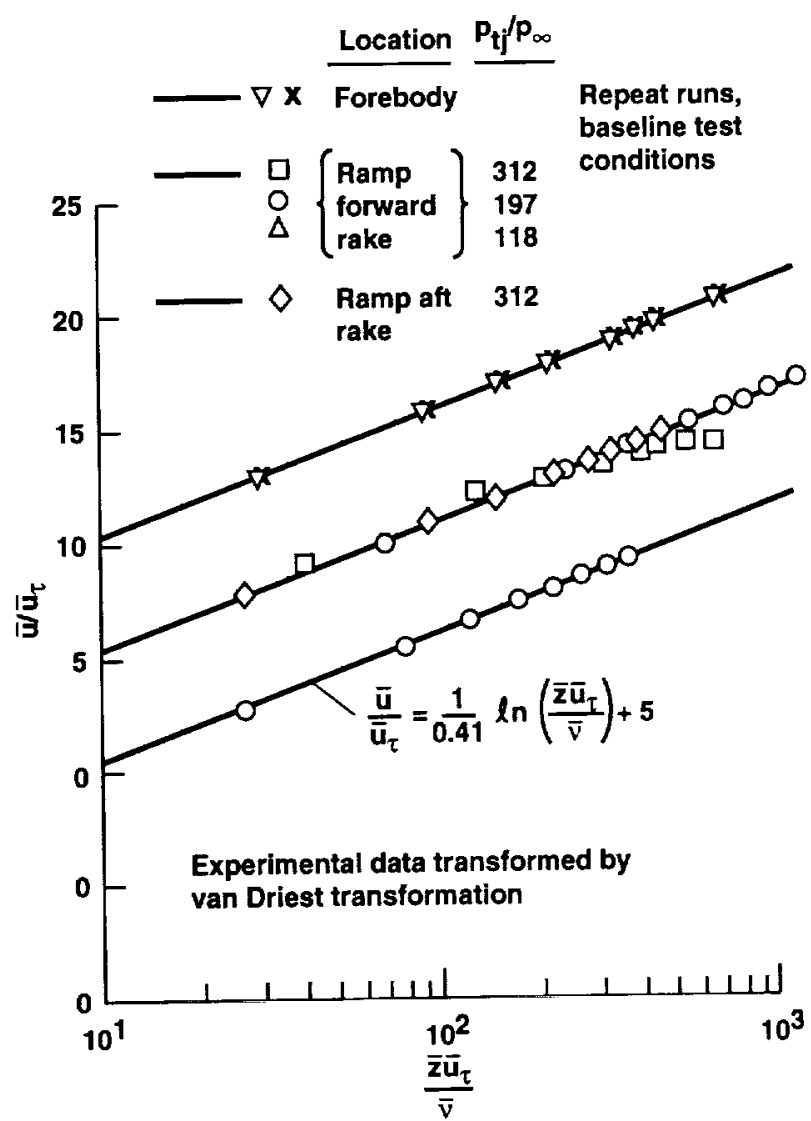

Figure 26. Forebody and ramp boundary-layer rake data in law-of-the-wall coordinates.

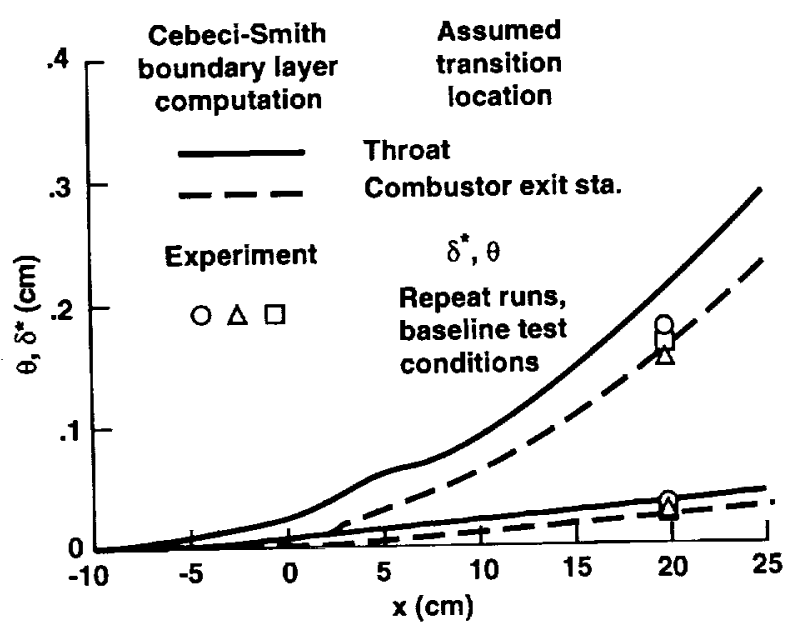

Figure 27. Ramp boundary-layer integral properties compared with 2-D boundary layer calculations.

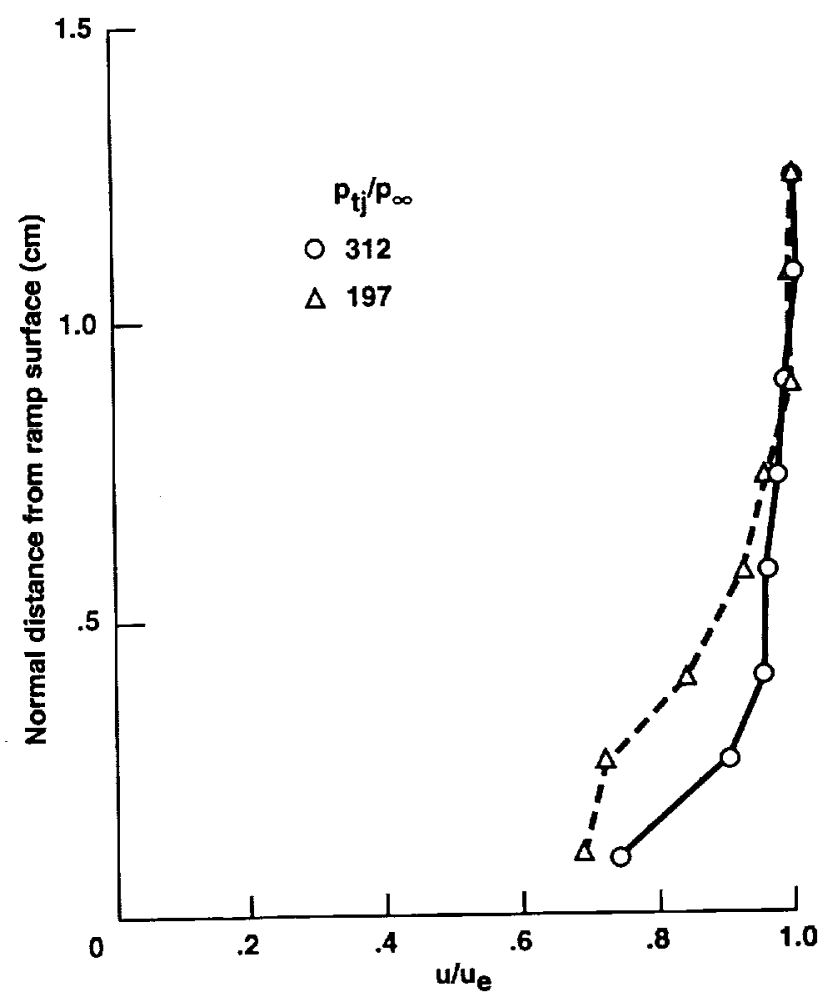

Figure 28. Boundary layer profiles from aft ramp rake. 


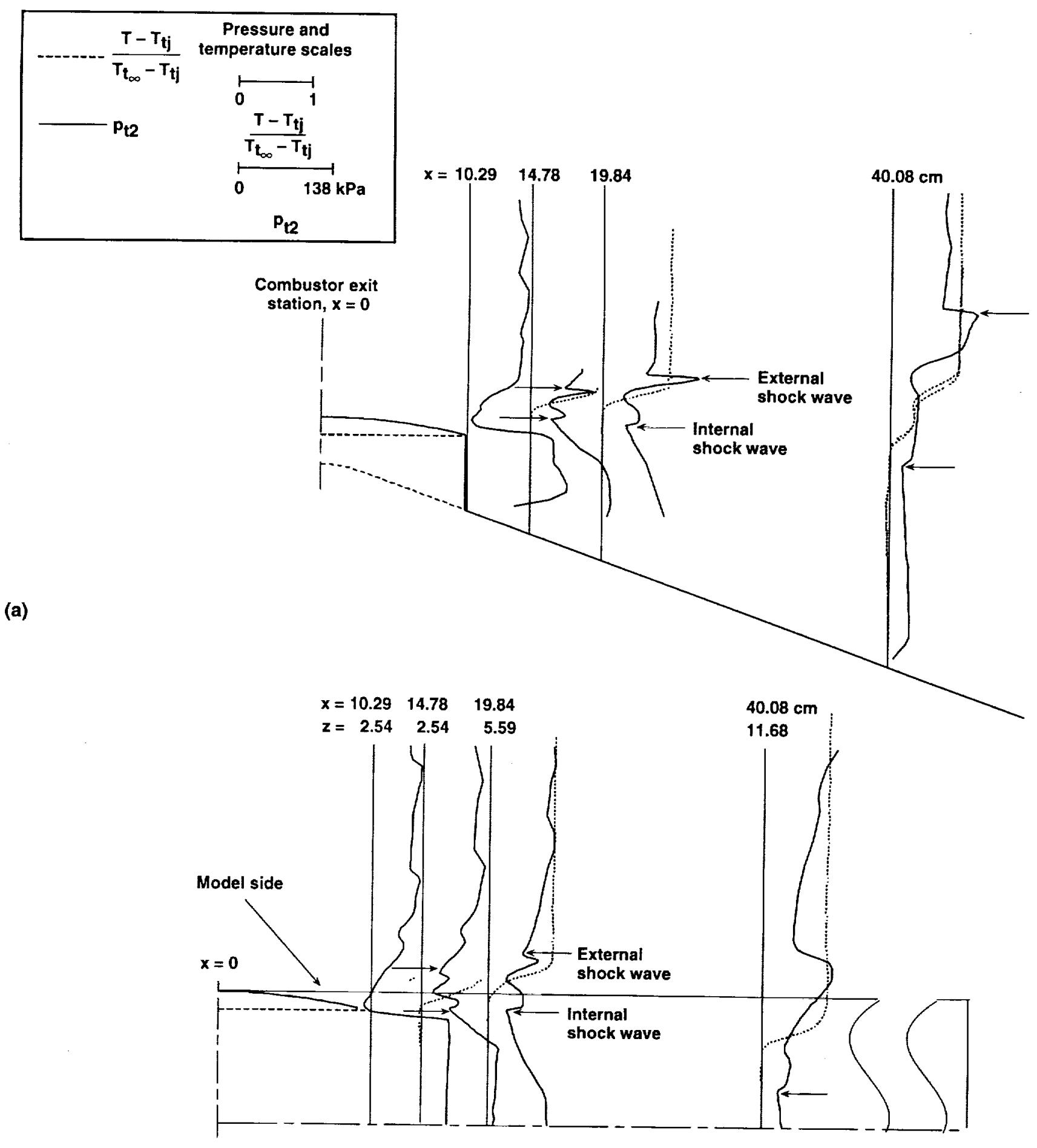

(b)

Figure 29. Composite view of impact-pressure and total-temperature distributions, baseline test conditions. (a) Surveys in symmetry plane, $y=0$; (b) spanwise surveys, $2.5<z<11.7$. 


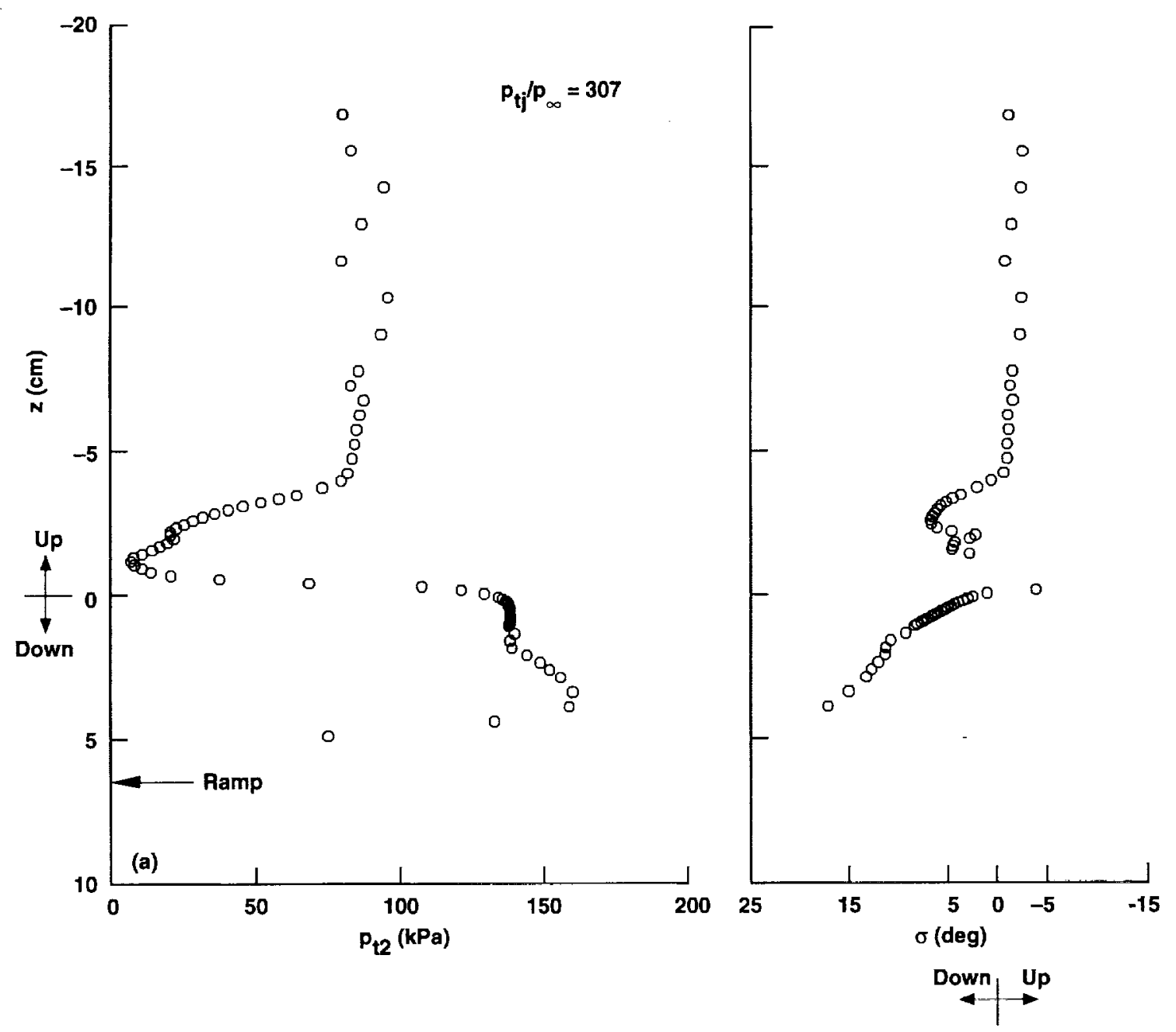

Figure 30. Impact-pressure and flow-angle data at cowl exit station, $x=10.29 \mathrm{~cm}$, baseline test conditions. (a) $P_{t 2}$ and $\sigma$ distributions at $y=0$. 


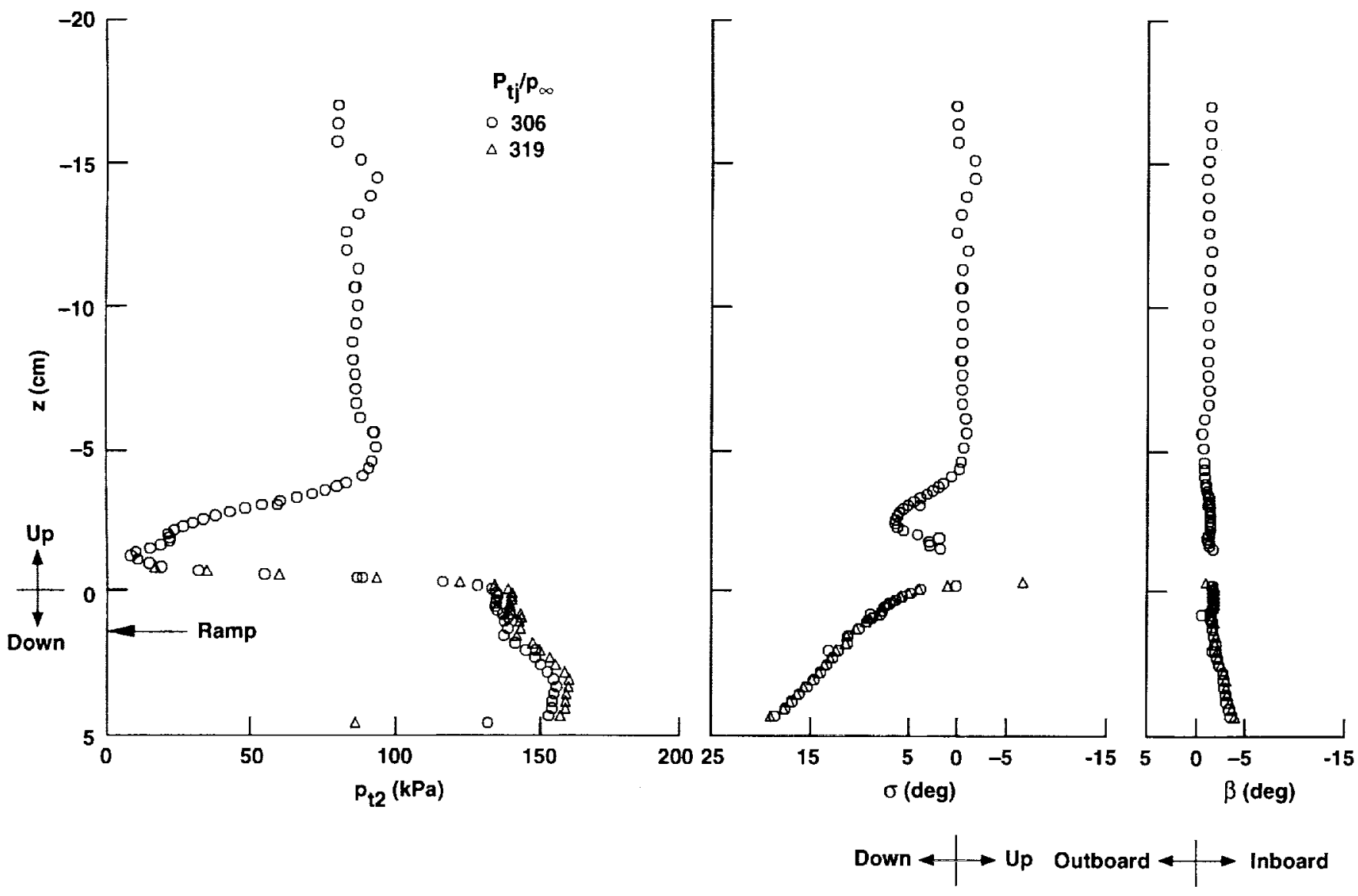

Figure 30. Continued. (b) $P_{t 2}, \sigma$, and $\beta$ distributions at $y=6.35 \mathrm{~cm}$. 

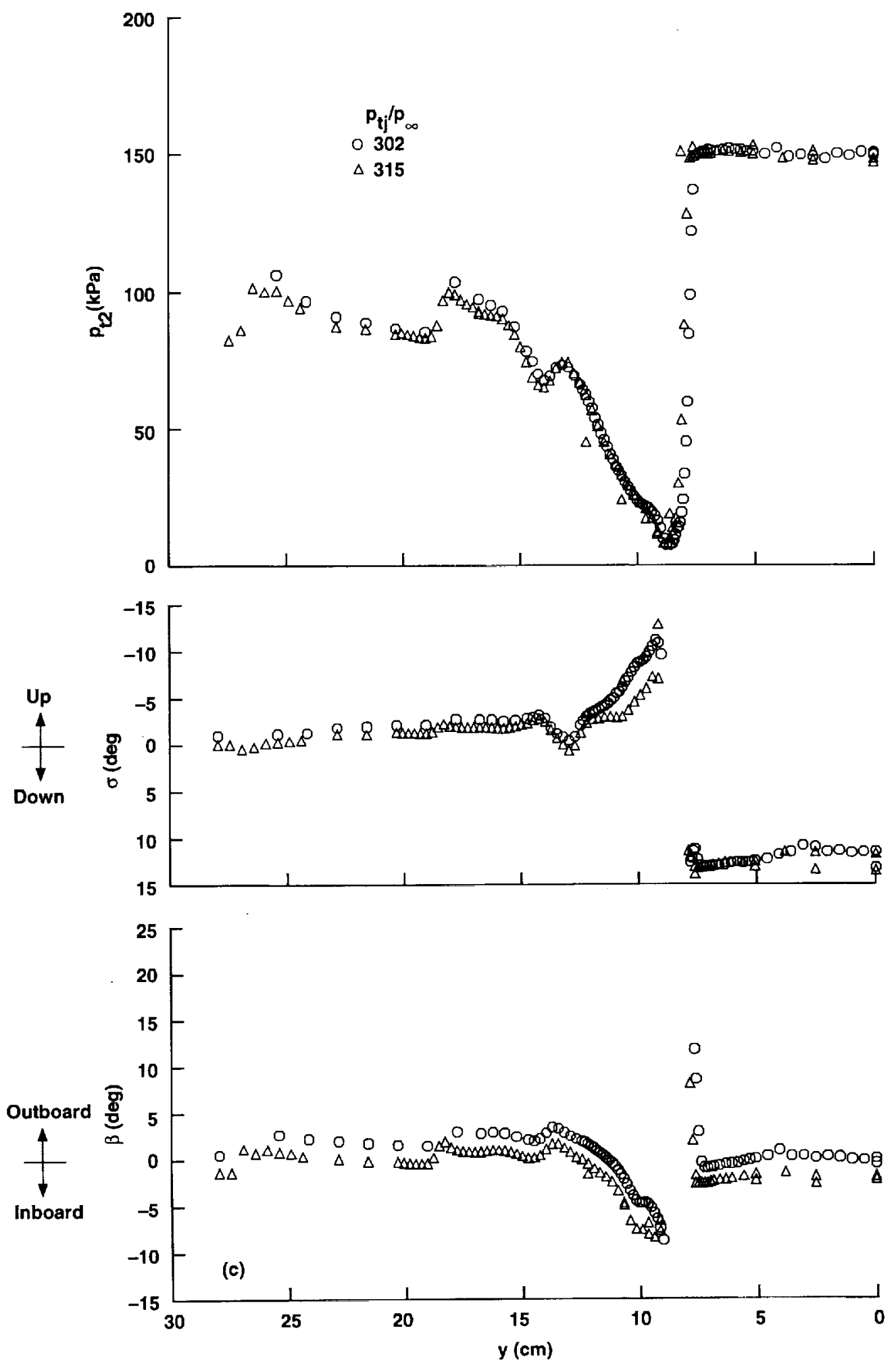

Figure 30. Concluded. (c) $P_{\uparrow 2}, \sigma$, and $\beta$ distributions at $z=2.54 \mathrm{~cm}$. 

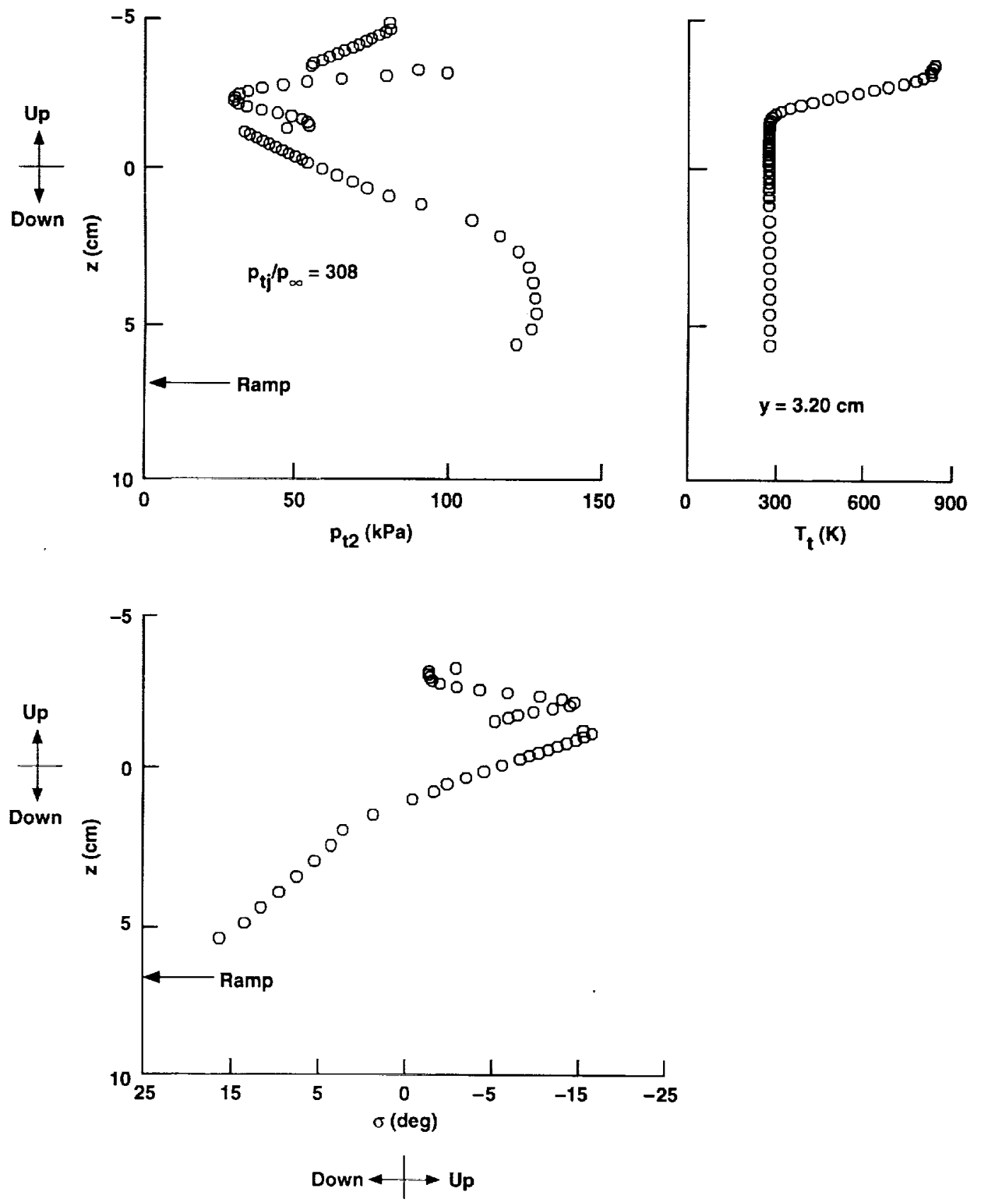

Figure 31. Impact-pressure, flow-angle, and total-temperature data at $x=14.78 \mathrm{~cm}$, baseline test conditions. (a) $P_{t 2}$ and $\sigma$ distributions at $y=0$. 

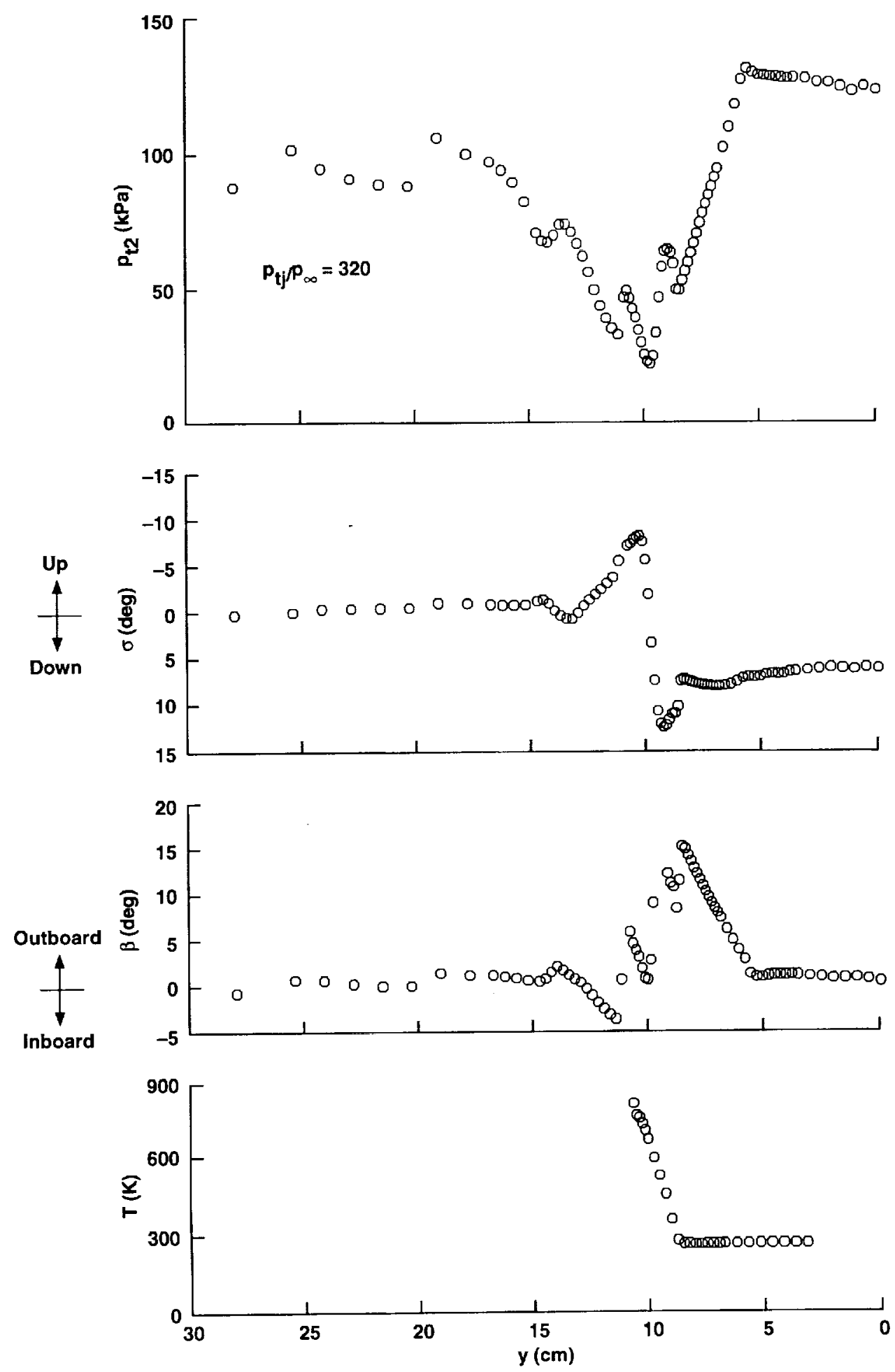

Figure 31. Concluded. (b) $P_{t 2}, \sigma, \beta$, and $T_{t}$ distributions at $z=2.54 \mathrm{~cm}$. 

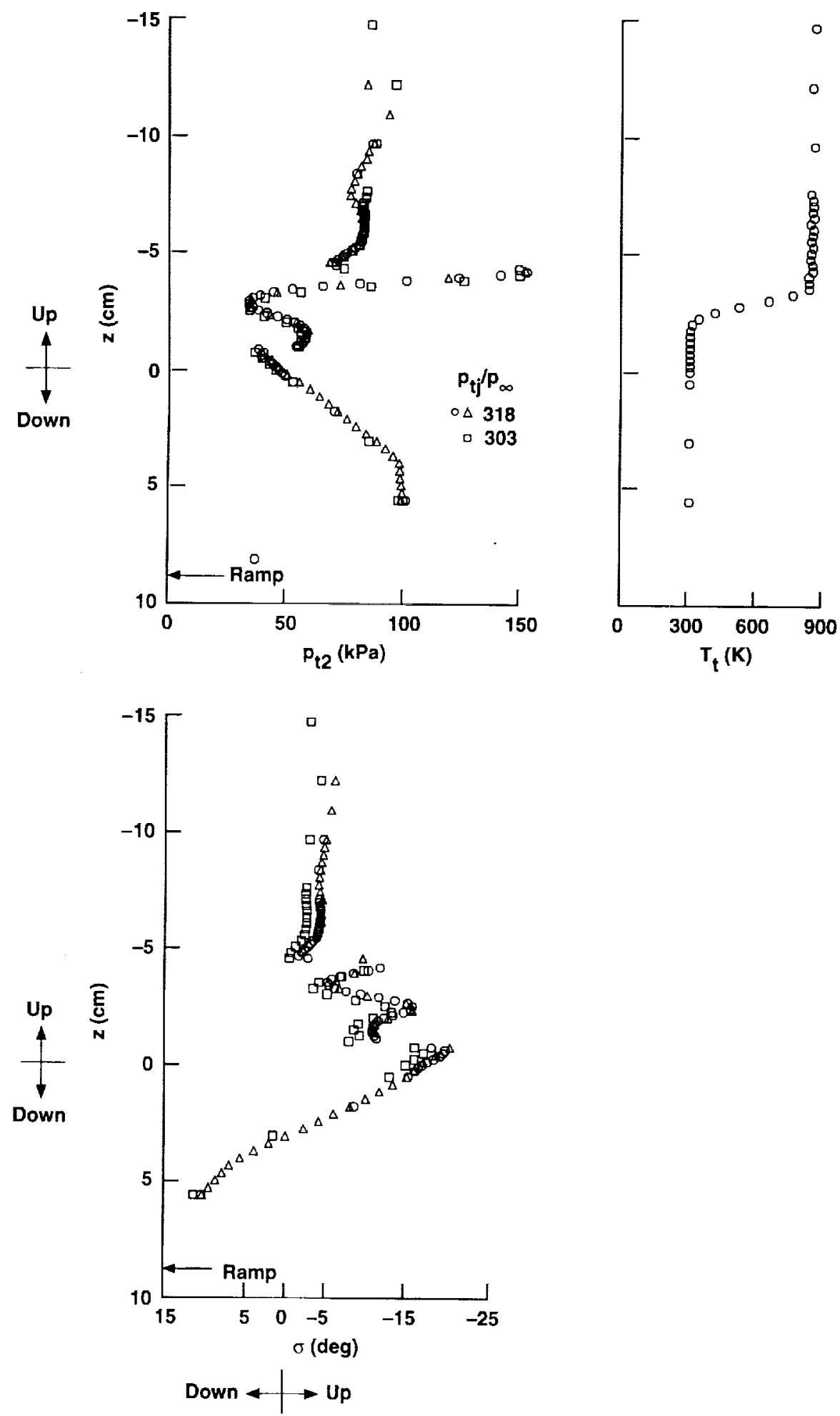

Figure 32. Impact-pressure, flow-angle, and total-temperature data at $x=19.84 \mathrm{~cm}$, baseline test conditions. (a) $P_{t 2}$ and $\sigma$ distributions at $y=0 ; T_{t}$ distribution at $y=3.20 \mathrm{~cm}$. 


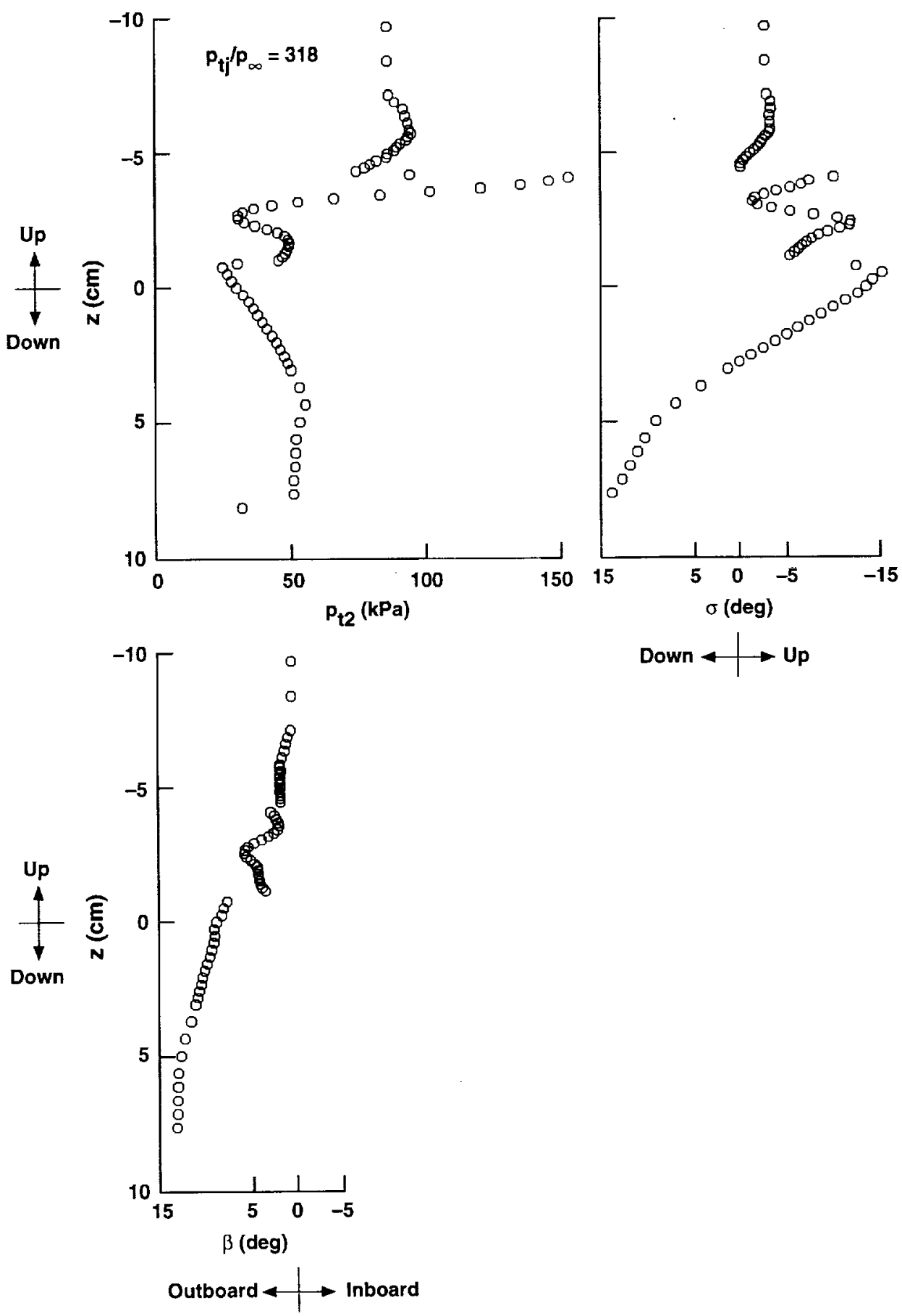

Figure 32. Continued. (b) $P_{t 2}, \sigma$, and $\beta$ distributions at $y=7.62 \mathrm{~cm}$. 

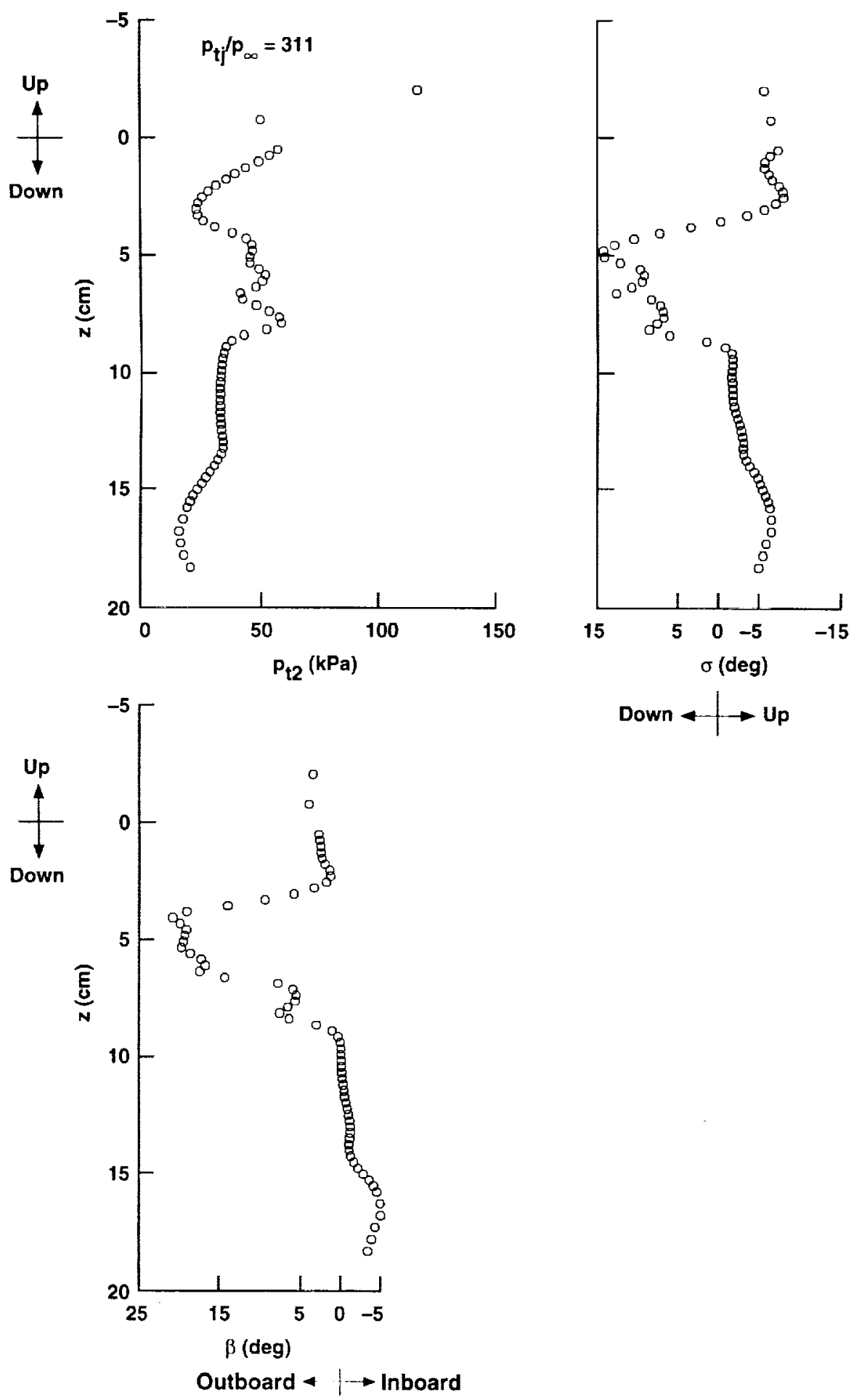

Figure 32. Continued. (c) $P_{t 2}, \sigma$, and $\beta$ distributions at $y=11.43 \mathrm{~cm}$. 

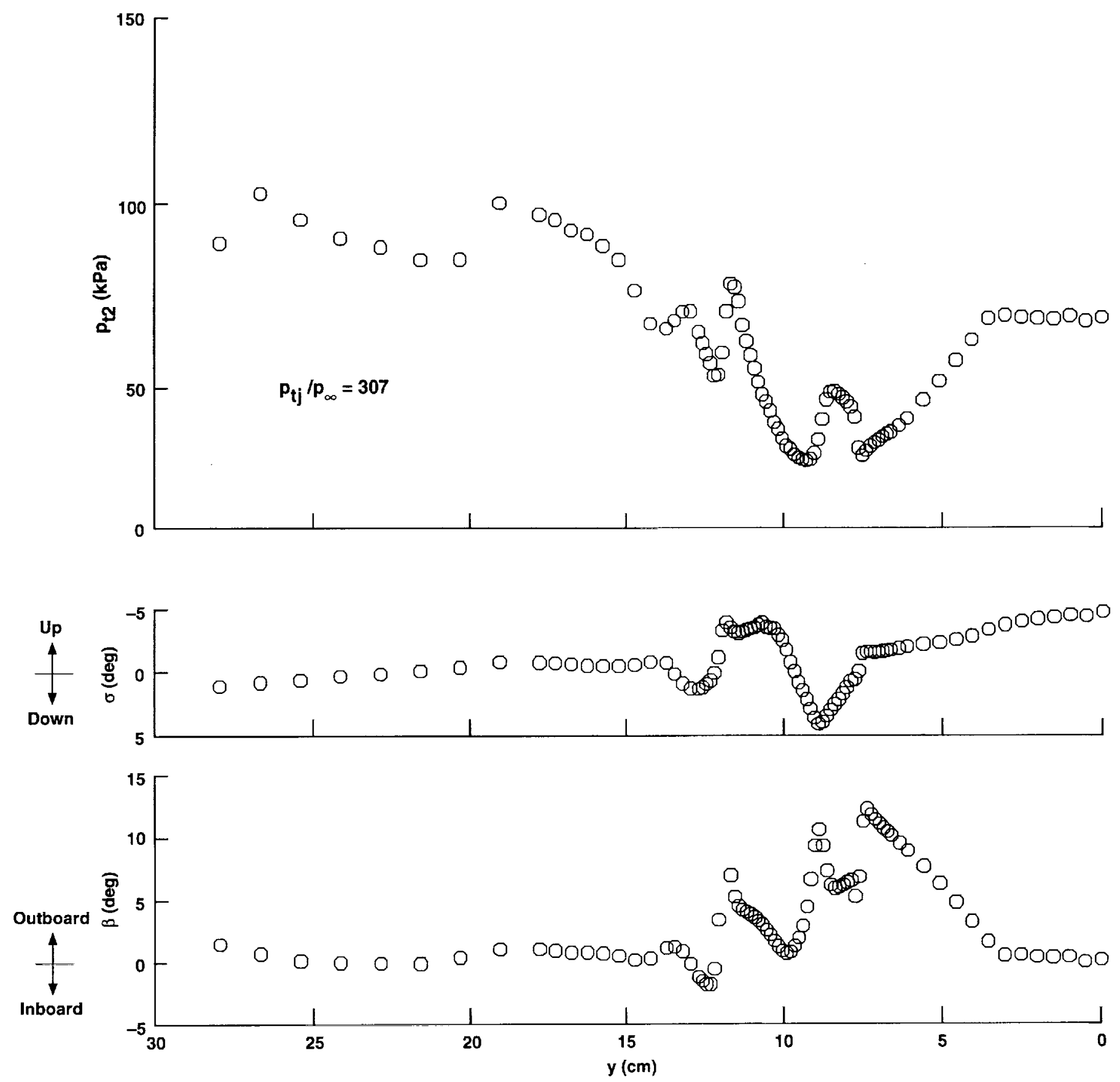

Figure 32. Continued. (d) $P_{t 2}, \sigma$, and $\beta$ distributions at $z=1.77 \mathrm{~cm}$. 


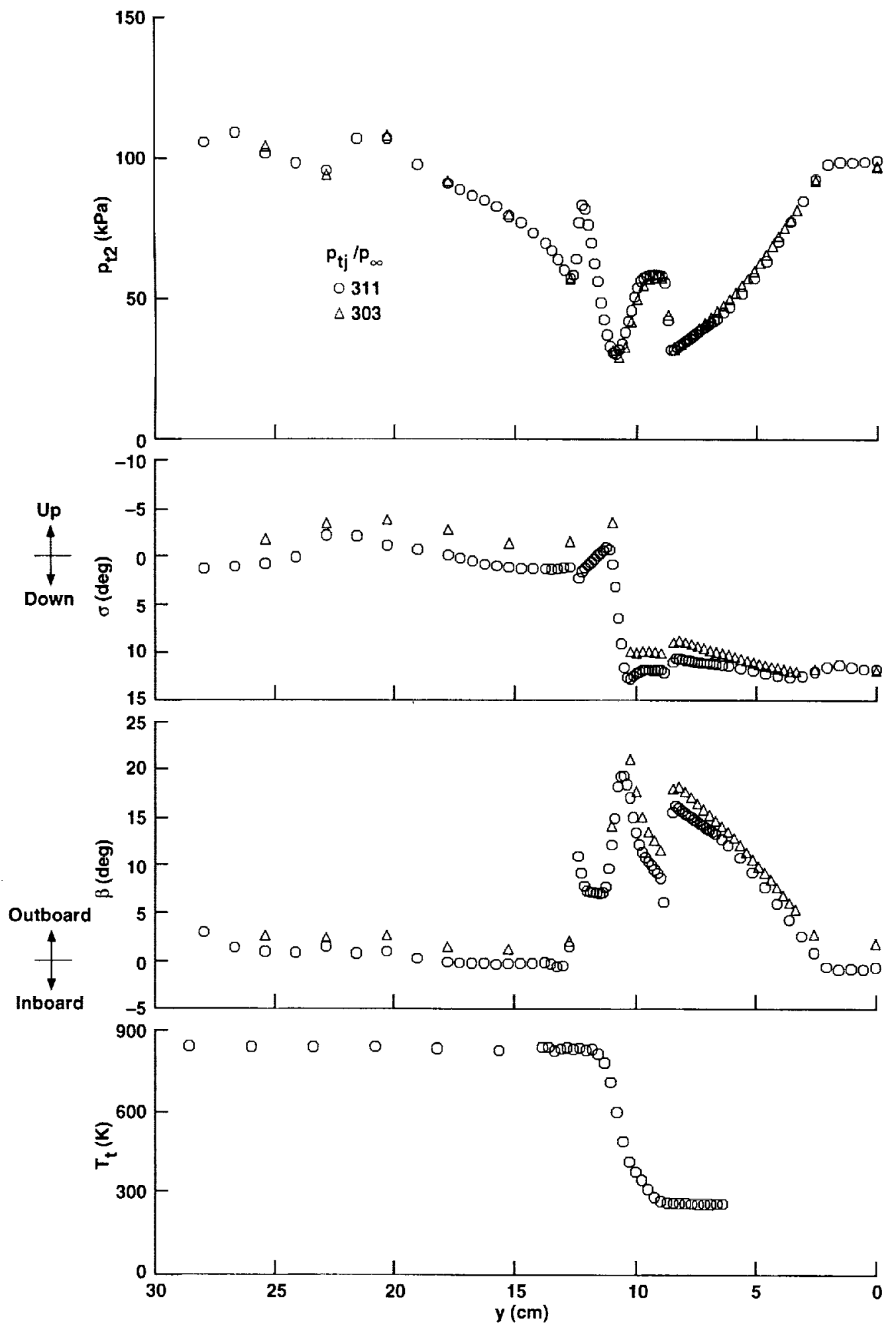

Figure 32. Concluded. (e) $P_{t 2}, \sigma, \beta$, and $T_{t}$ distributions at $z=5.58 \mathrm{~cm}$. 

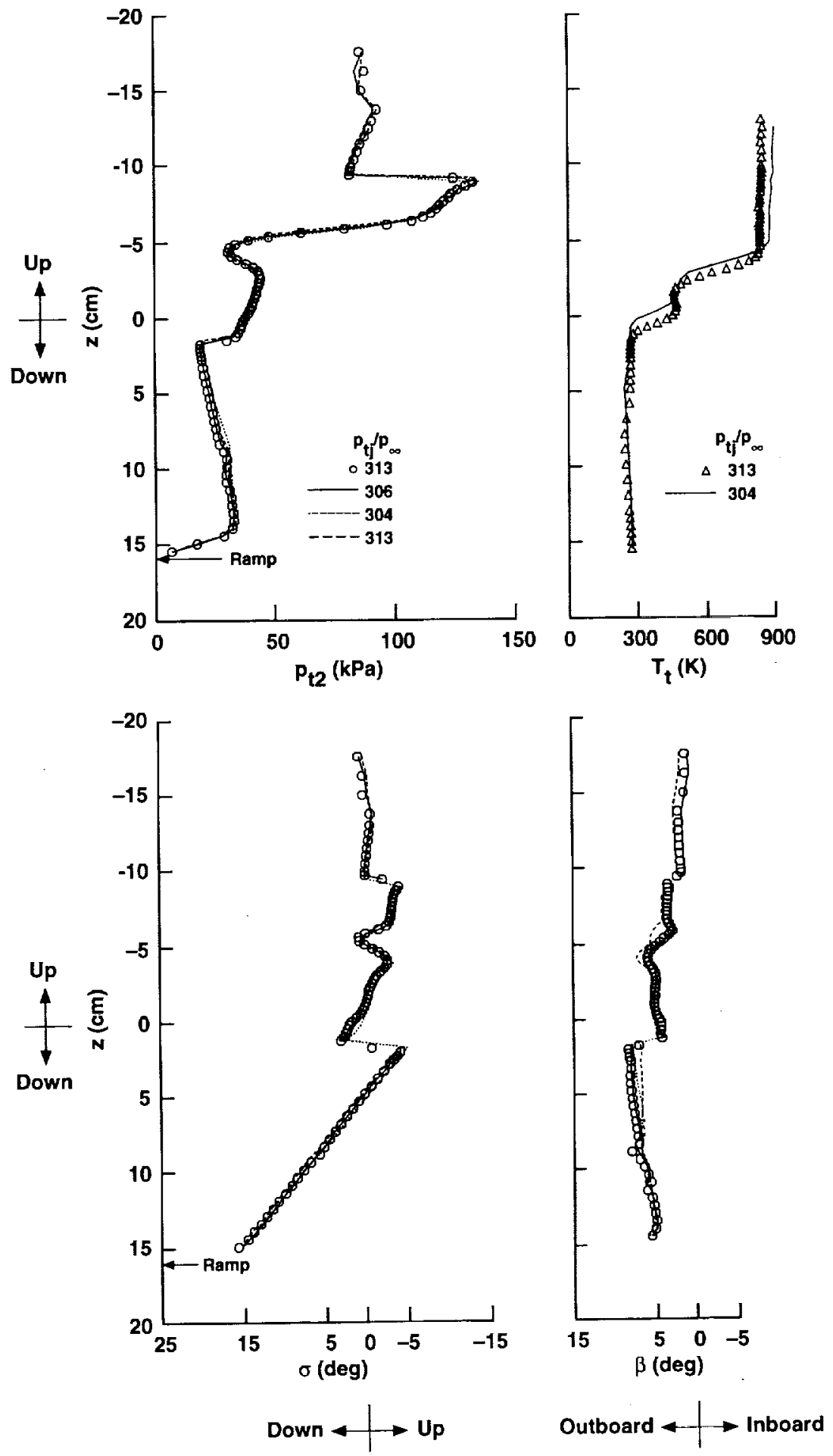

Figure 33. Impact-pressure, flow-angle, and total-temperature data at $x=40.08 \mathrm{~cm}$, baseline test conditions. (a) $P_{t 2}, \sigma, \beta$, and $T_{t}$ distributions at $y=0 ; T_{t}$ distribution at $y=3.20 \mathrm{~cm}$. 

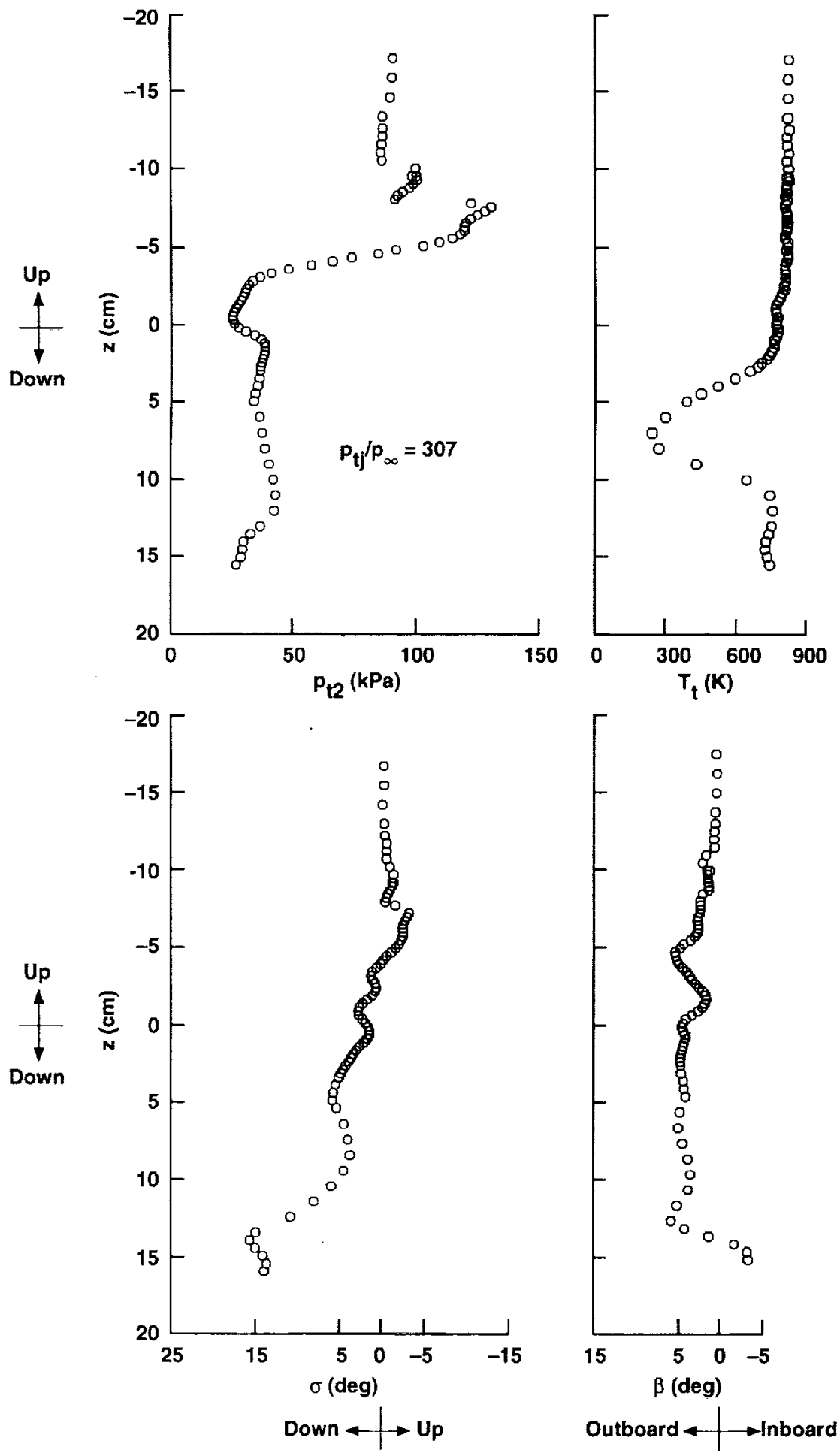

Figure 33. Continued. (b) $P_{t 2}, \sigma$, and $\beta$ distributions at $y=6.35 \mathrm{~cm}$; $T_{t}$ distribution at $y=9.55 \mathrm{~cm}$. 

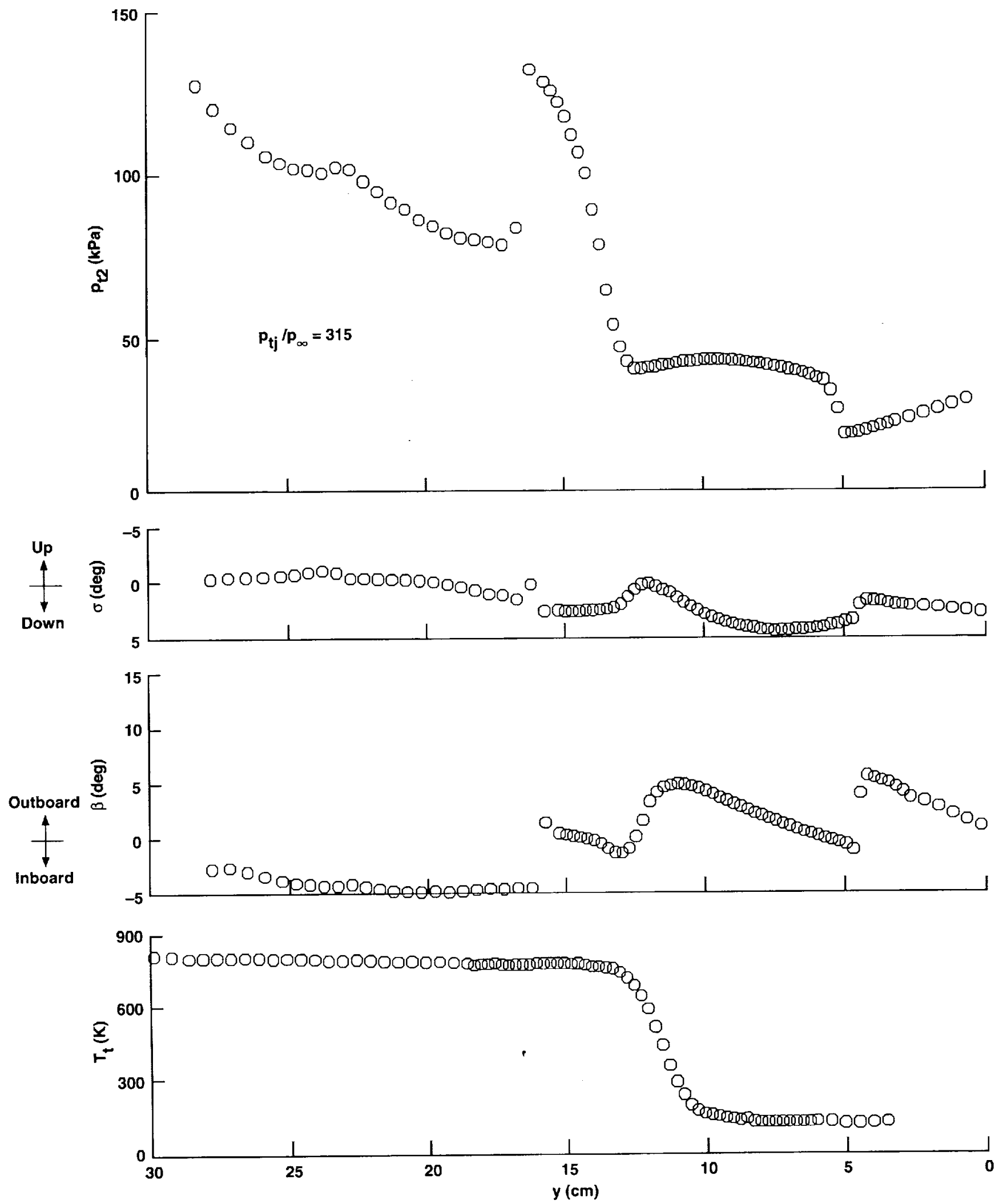

Figure 33. Continued. (c) $P_{t 2}, \sigma, \beta$, and $T_{f}$ distributions at $z=6.85 \mathrm{~cm}$. 


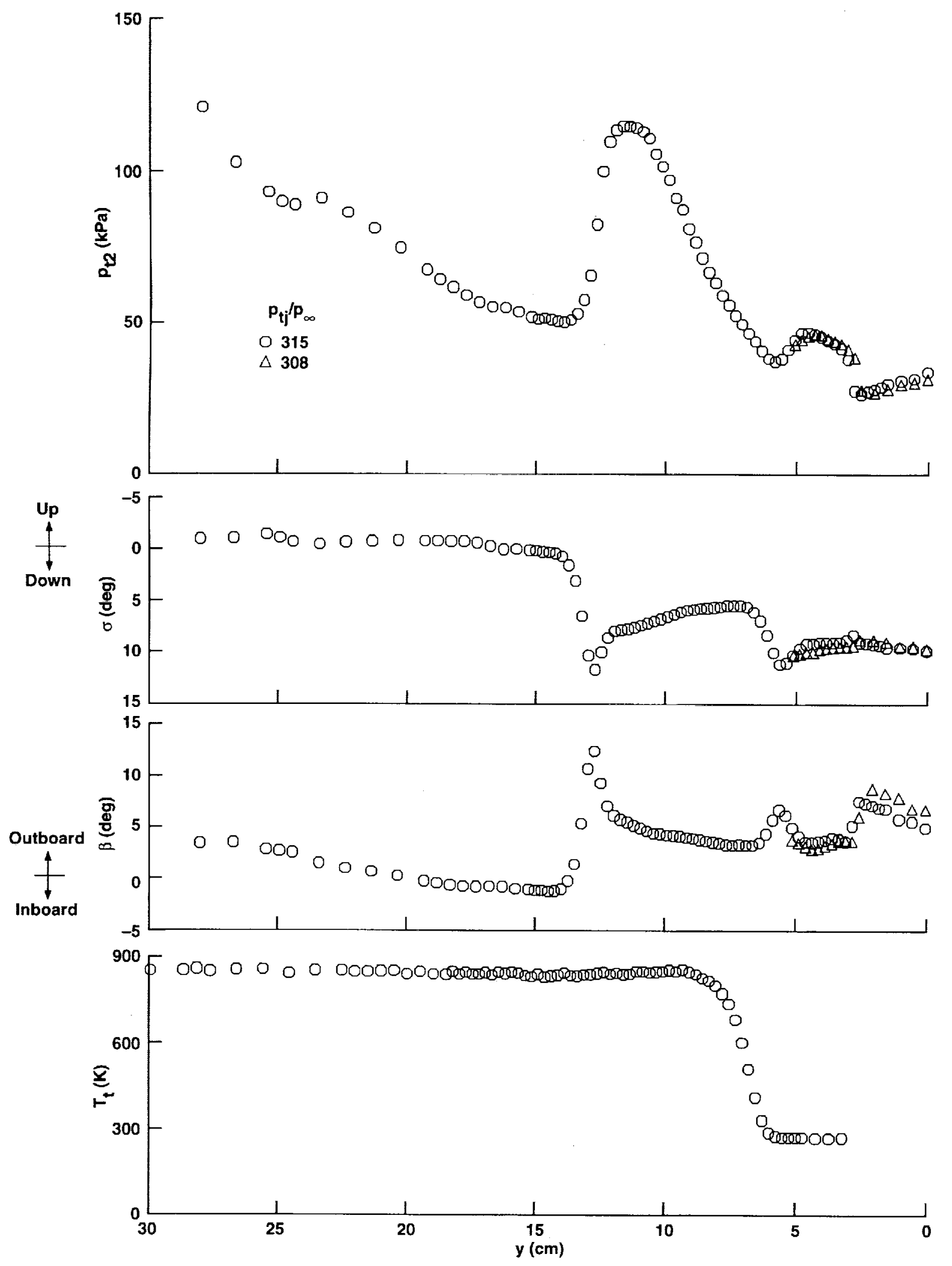

Figure 33. Concluded. (d) $P_{t 2}, \sigma, \beta$, and $T_{t}$ distributions at $z=11.67 \mathrm{~cm}$. 


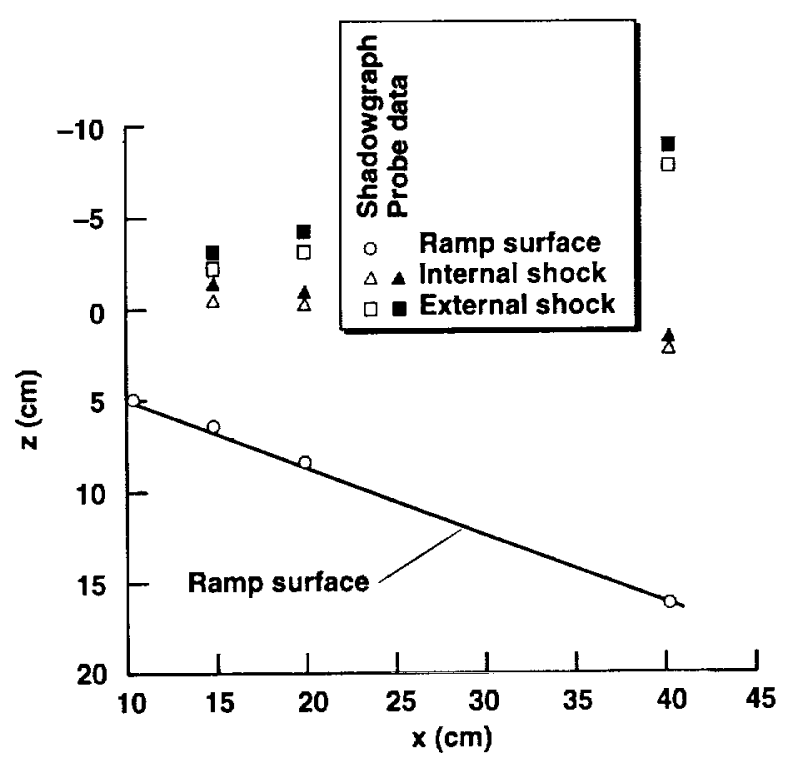

Figure 34. Shock-wave and ramp-surface locations obtained from a shadowgraph photograph and probe data.

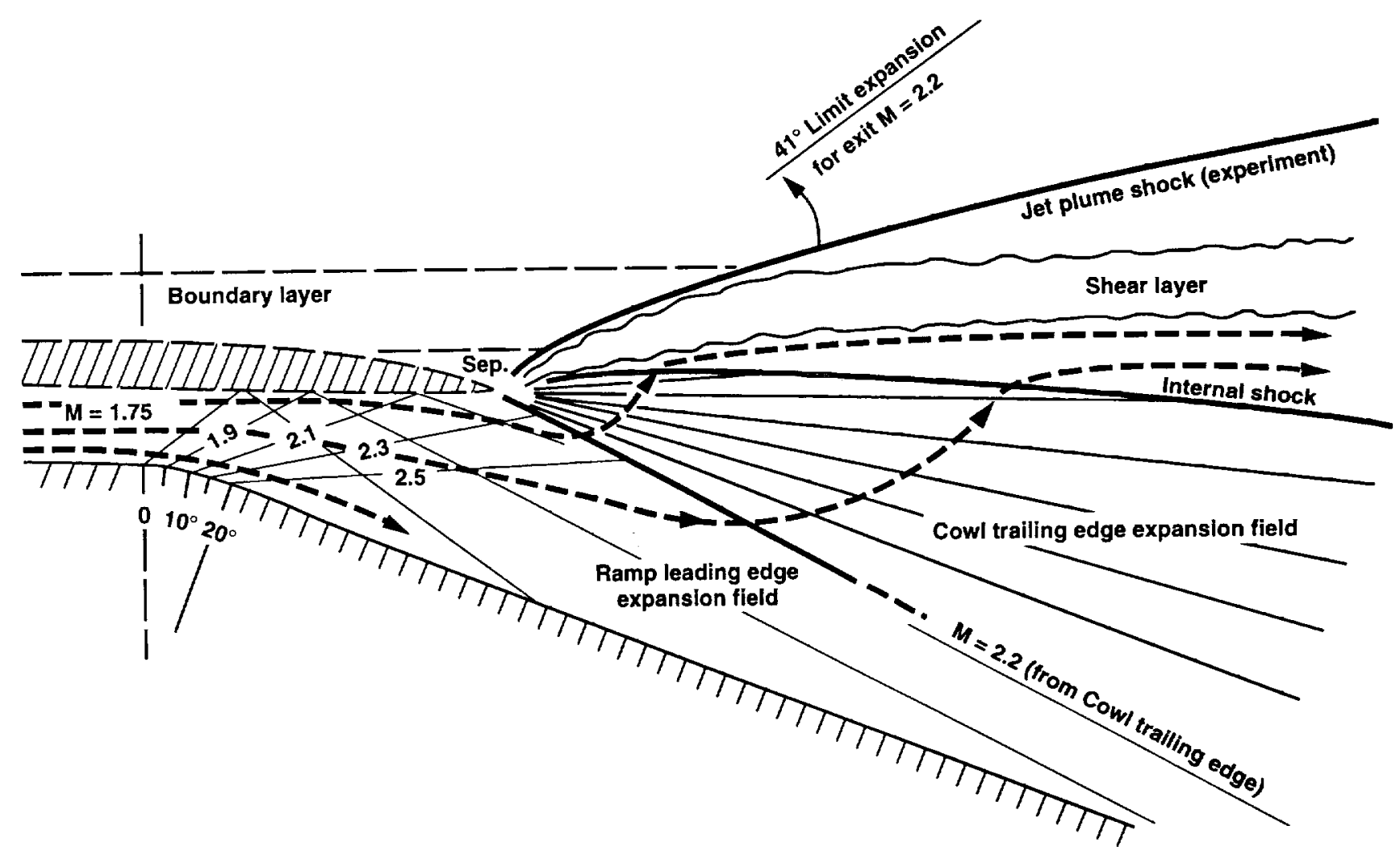

Figure 35. Schematic of vertical cross section and wave diagram of internal nozzle, cowl, and jet plume at baseline test conditions. 


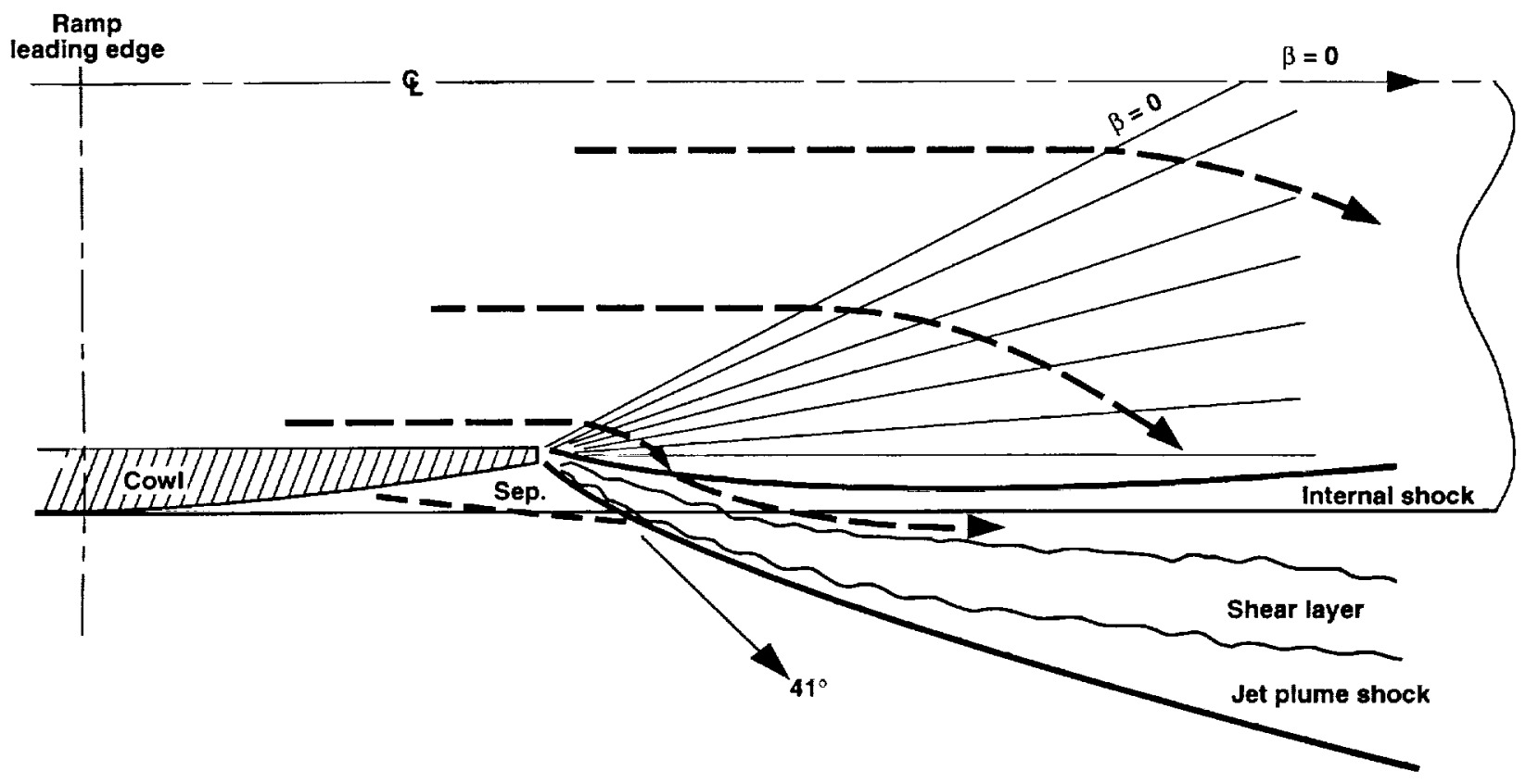

Figure 36. Schematic of horizontal cross section (planform) and wave diagram of cowl and jet plume at baseline test conditions.

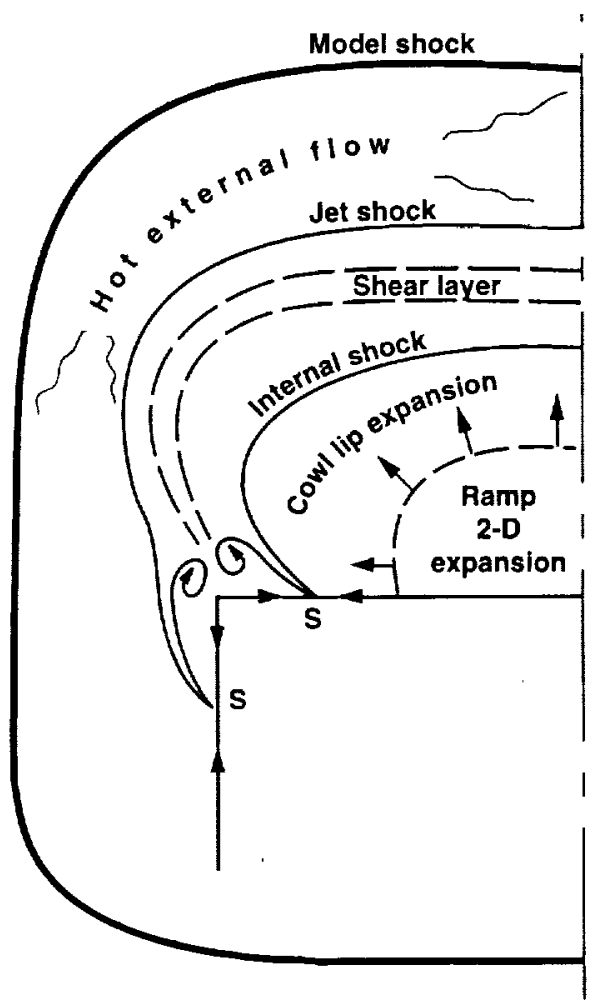

Figure 37. Schematic of transverse cross section of jet plume. 

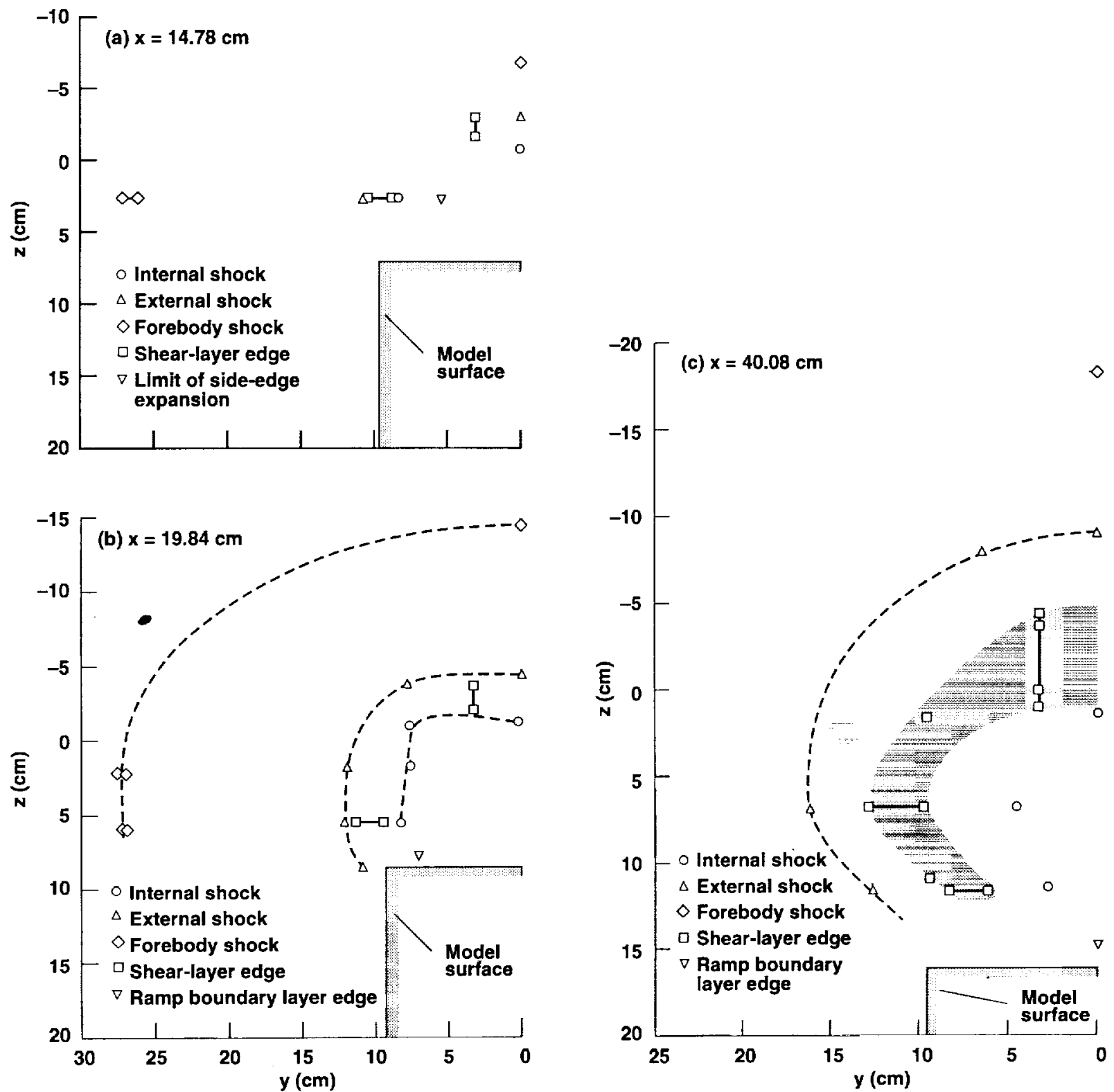

Figure 38. Shock-wave and shear-layer locations obtained from probe data, baseline test conditions. (a) $x=14.78 \mathrm{~cm}$; (b) $x=19.84 \mathrm{~cm}$; (c) $x=40.08 \mathrm{~cm}$. 

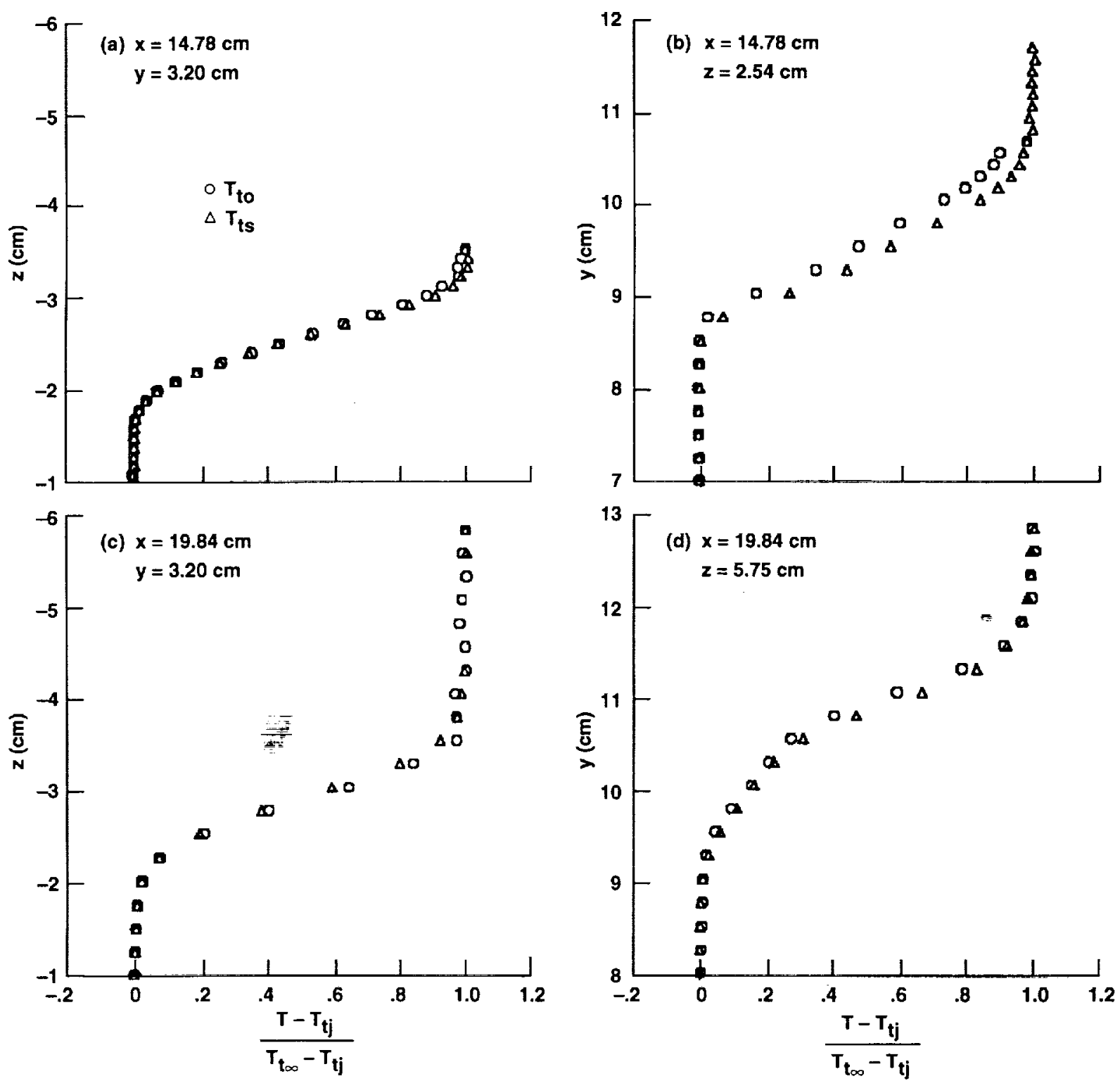

Figure 39. Normalized sensor and support temperature distributions, baseline test conditions. (a) $x=14.78 \mathrm{~cm}$, $y=3.20 \mathrm{~cm}$; (b) $x=14.78 \mathrm{~cm}, z=2.54 \mathrm{~cm}$; (c) $x=19.84 \mathrm{~cm}, y=3.20 \mathrm{~cm}$; (d) $x=19.84 \mathrm{~cm}, z=5.75 \mathrm{~cm}$. 

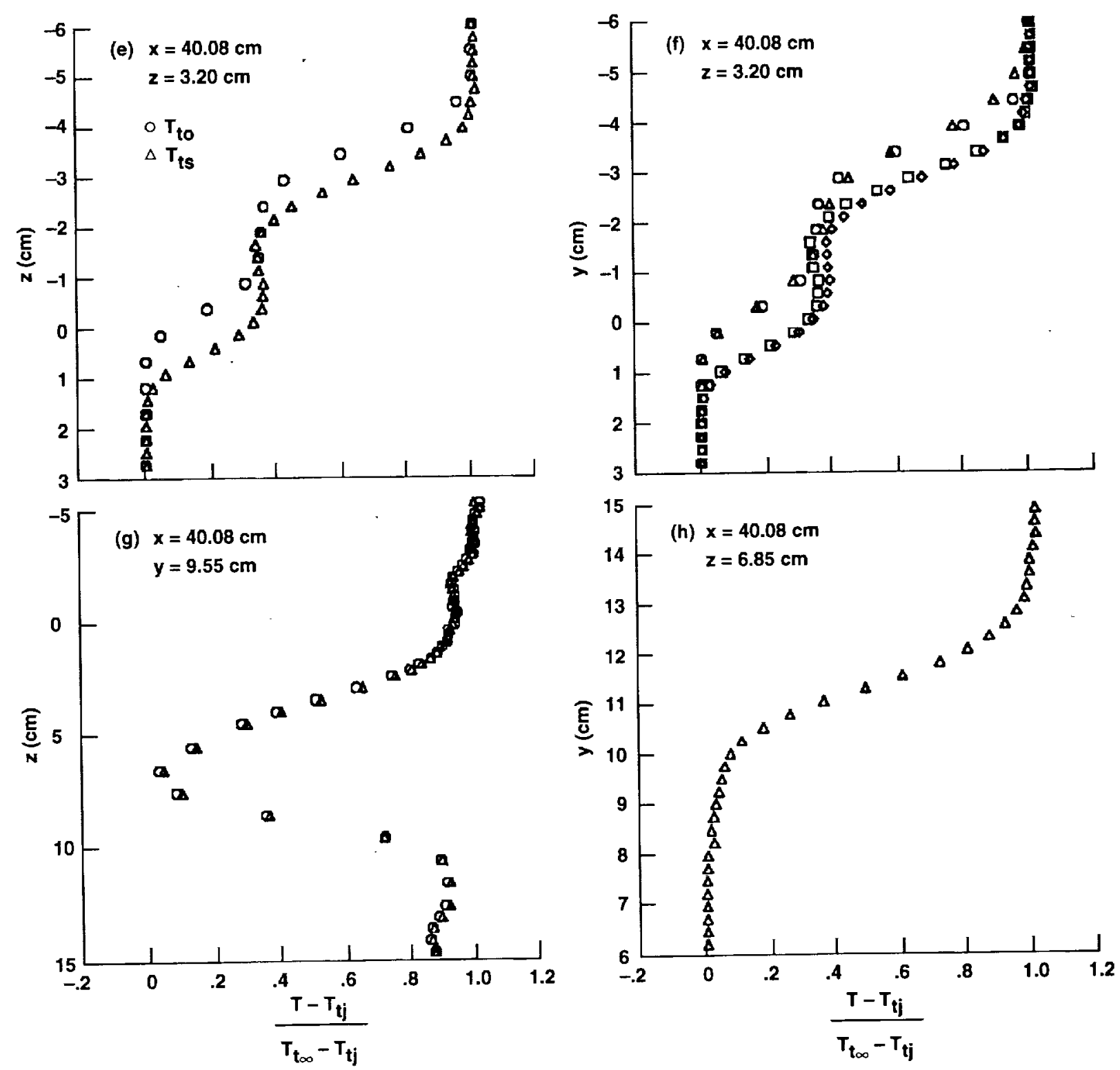

Figure 39. Continued. (e) $x=40.08 \mathrm{~cm}, z=3.20 \mathrm{~cm} ;(f) x=40.08 \mathrm{~cm}, y=3.20 \mathrm{~cm} ;(g) x=40.08 \mathrm{~cm}, y=9.55 \mathrm{~cm}$; (h) $x=40.08 \mathrm{~cm}, z=6.85 \mathrm{~cm}$. 


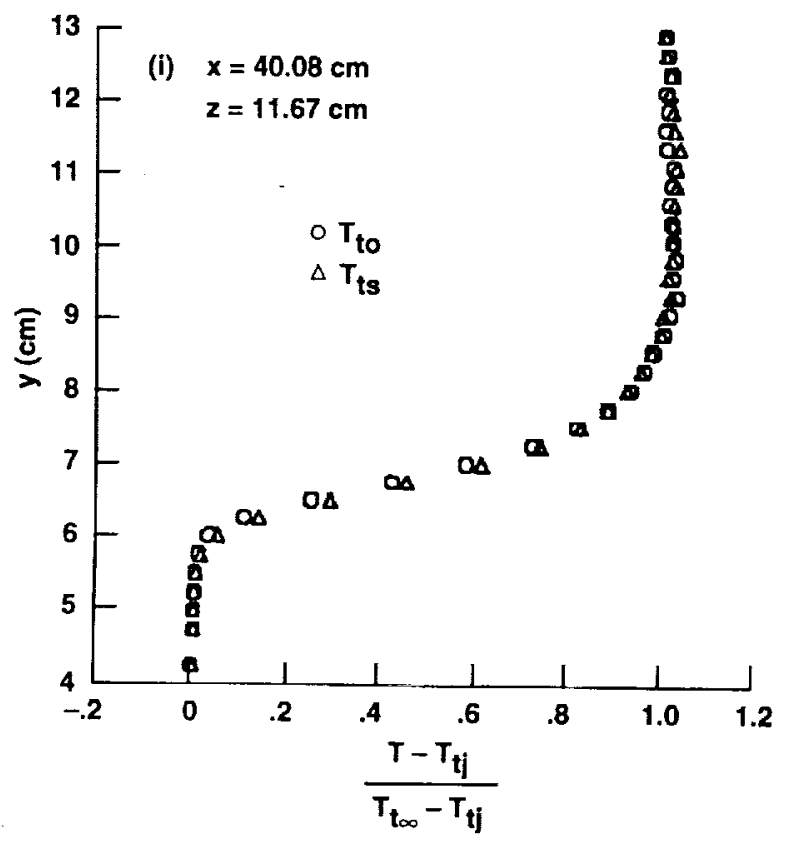

Figure 39. Concluded. (i) $x=40.08 \mathrm{~cm}, z=11.67 \mathrm{~cm}$.

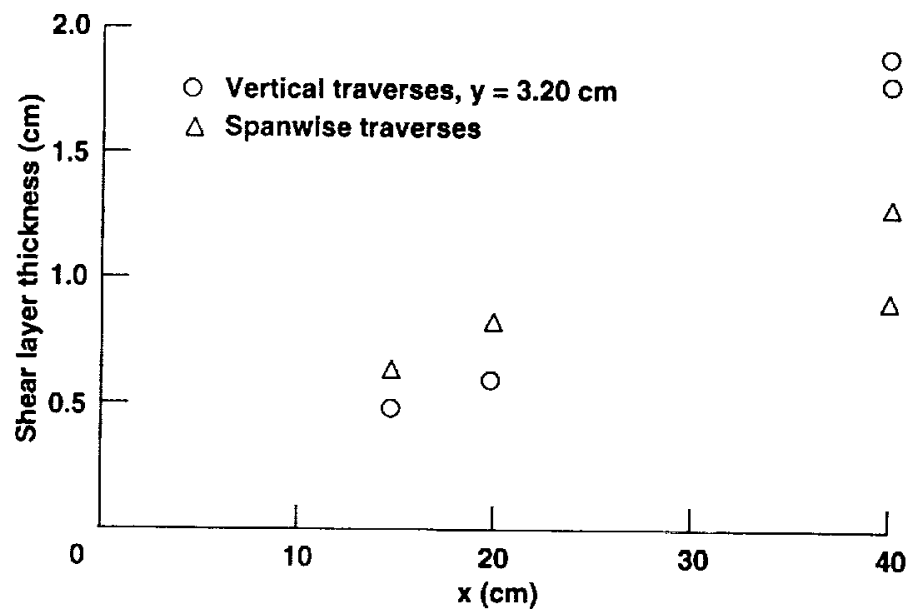

Figure 40. Shear-layer thickness obtained from thermocouple-probe surveys. 


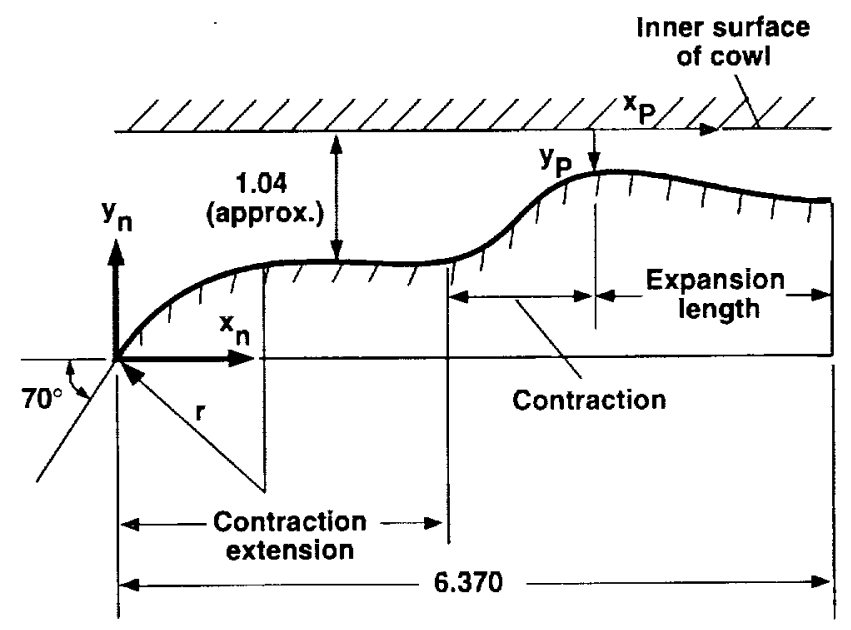

Figure 41. Internal nozzle cross section. 

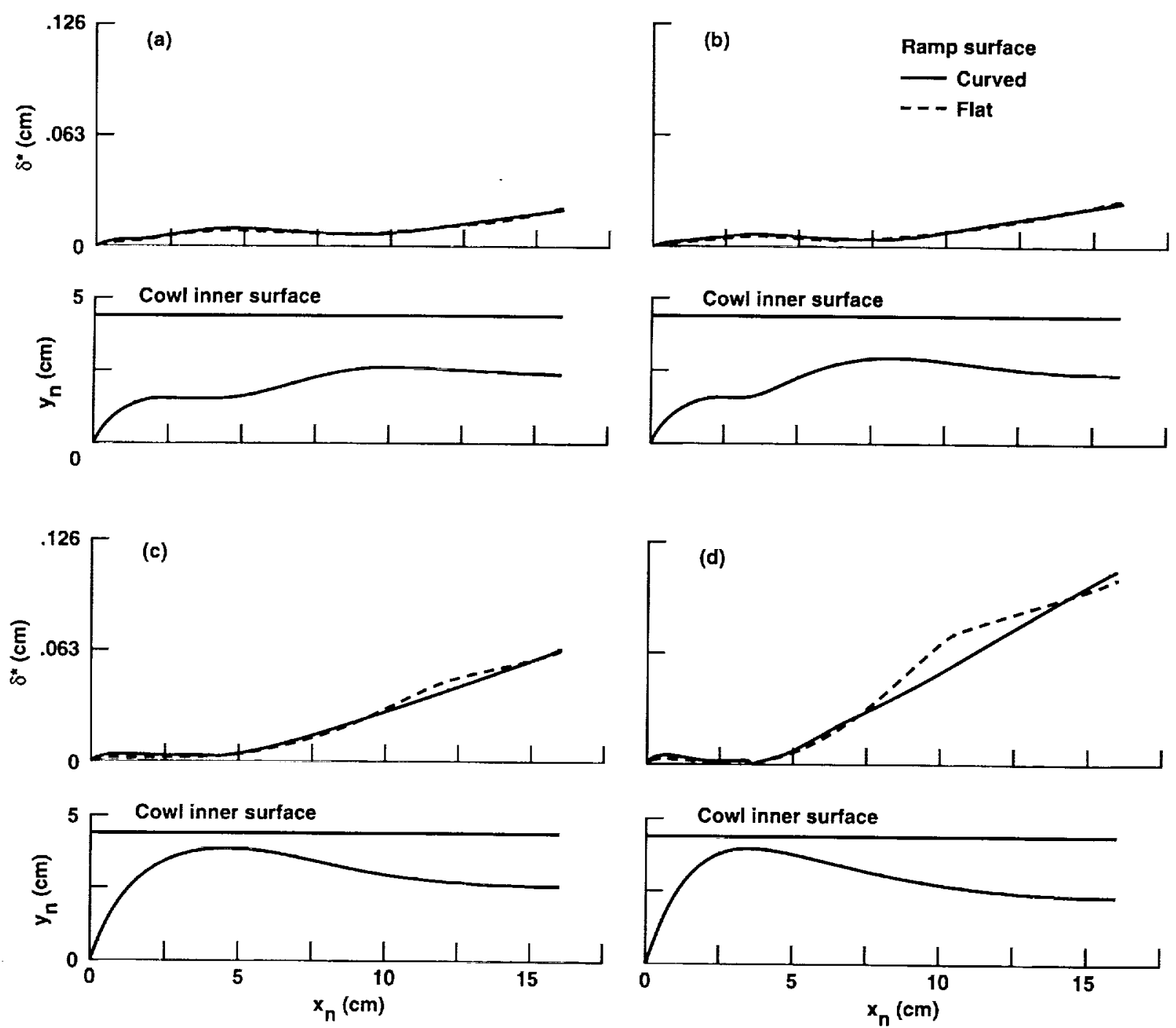

Figure 42. Internal nozzle contours and displacement-thickness distributions. (a) $M_{j}=1.4 ;$ (b) $M_{j}=1.74$; (c) $M_{j}=2.60 ;$ (d) $M_{j}=3.40$. 

Public reporting burden for this collection of information is estimated to average 1 hour per response, including the time for reviewing instructions, searching existing data sources, gathering and maintaining the data needed, and completing and reviewing the colfection of information. Send comments regarding this burden estimate or any other aspect of this collection of information, including suggestions for reducing this burden, to Washington Headquarters Services, Directorate for information Operations and Reports, 1215 Jefferson Davis Highway, Suite 1204, Arlington, VA 22202-4302, and to the Office of Management and Budget, Paperwork Reduction Project (0704-0188), Washington, DC 20503.

\begin{tabular}{l|l|l|l} 
1. AGENCY USE ONLY (Leave blank) & 2. REPORT DATE & 3. REPORT TYPE AND DATES COVERED
\end{tabular}

4. TITLE AND SUBTITLE March 1995 Technical Memorandum

Experimental Results for a Hypersonic Nozzle/Afterbody Flow Field

6. AUTHOR(S)

$505-70-62$

Frank W. Spaid,* Earl R. Keener, and Frank C. L. Hui

7. PERforming organization NAME(S) AND ADDRESS(ES)

8. PERFORMING ORgANIZATION REPORT NUMBER

Ames Research Center

Moffett Field, CA 94035-1000

A-94119

9. SPONSORING/MONITORING AGENCY NAME(S) AND ADDRESS(ES)

10. SPONSORING/MONITORING AGENCY REPORT NUMBER

National Aeronautics and Space Administration

Washington, DC 20546-0001

NASA TM-4638

11. SUPPLEMENTARY NOTES

Point of Contact: Frank C. L. Hui, Ames Research Center, MS 229-4, Moffett Field, CA 94035-1000 (415) 604-5395

*McDonnell Douglas Corporation, St. Louis, Missouri; ${ }^{\dagger}$ Eloret Institute, Palo Alto, California 12a. DISTRIBUTION/AVAILABILITY STATEMENT

Unclassified - Unlimited

Subject Category 02

Available from the NASA Center for Aerospace Information,

800 Elkridge Landing Road, Linthicum Heights, MD 21090; (301) 621-0390

13. ABSTRACT (Maximum 200 words)

This study was conducted to experimentally characterize the flow field created by the interaction of a single-expansion-ramp-nozzle (SERN) flow with a hypersonic external stream. Data were obtained from a generic nozzle/afterbody model in the 3.5-Foot Hypersonic Wind Tunnel at the NASA Ames Research Center, in a cooperative experimental program involving Ames and McDonnell Douglas Aerospace. The model design and test planning were performed in close cooperation with members of the Ames computational fluid dynamics (CFD) team for the National Aero-Space Plane (NASP) program. This paper presents experimental results consisting of oil-flow and shadowgraph flow-visualization photographs, afterbody surface-pressure distributions, rake boundary-layer measurements, Preston-tube skin-friction measurements, and flow field surveys with five-hole and thermocouple probes. The probe data consist of impact pressure, flow direction, and total temperature profiles in the interaction flow field.

14. SUBJECT TERMS

Hypersonic nozzle, Hypersonic flow interaction, NASP

15. NUMBER OF PAGES

104

Hypersonic nozzle, Hypersonic flow interaction, NASP

16. PRICE CODE

A06

\begin{tabular}{l|l}
\hline $\begin{array}{l}\text { 17. SECURITY CLASSIFICATION } \\
\text { OF REPORT } \\
\text { Unclassified }\end{array}$ & $\begin{array}{l}\text { 18. SECURITY CLASSIFICATION } \\
\text { OF THIS PAGE } \\
\text { Unclassified }\end{array}$ \\
\hline
\end{tabular}

19. SECURITY CLASSIFICATION OF ABSTRACT 

